SLAC-PUB-8569

BABAR-PUB-01/08

hep-ex/0105044

April, 2001

\title{
The BABAR Detector
}

\author{
The BABAR Collaboration
}

\begin{abstract}
$B A B A R$, the detector for the SLAC PEP-II asymmetric $e^{+} e^{-} B$ Factory operating at the $\Upsilon(4 S)$ resonance, was designed to allow comprehensive studies of $C P$-violation in $B$-meson decays. Charged particle tracks are measured in a multi-layer silicon vertex tracker surrounded by a cylindrical wire drift chamber. Electromagentic showers from electrons and photons are detected in an array of CsI crystals located just inside the solenoidal coil of a superconducting magnet. Muons and neutral hadrons are identified by arrays of resistive plate chambers inserted into gaps in the steel flux return of the magnet. Charged hadrons are identified by $d E / d x$ measurements in the tracking detectors and in a ring-imaging Cherenkov detector surrounding the drift chamber. The trigger, data acquisition and data-monitoring systems, VME- and network-based, are controlled by custom-designed online software. Details of the layout and performance of the detector components and their associated electronics and software are presented.
\end{abstract}

Submitted to Nuclear Instruments and Methods

Stanford Linear Accelerator Center, Stanford University, Stanford, CA 94309

Work supported in part by Department of Energy contract DE-AC03-76SF00515. 
The BABAR Collaboration

B. Aubert, A. Bazan, A. Boucham, D. Boutigny, I. De Bonis, J. Favier, J.-M. Gaillard, A. Jeremie, Y. Karyotakis, T. Le Flour, J.P. Lees, S. Lieunard, P. Petitpas, P. Robbe, V. Tisserand, K. Zachariadou Laboratoire de Physique des Particules, F-74941 Annecy-le-Vieux, France

A. Palano

Università di Bari, Dipartimento di Fisica and INFN, I-70126 Bari, Italy

G.P. Chen, J.C. Chen, N.D. Qi, G. Rong, P. Wang, Y.S. Zhu

Institute of High Energy Physics, Beijing 100039, China

G. Eigen, P.L. Reinertsen, B. Stugu

University of Bergen, N-5007 Bergen, Norway

B. Abbott, G.S. Abrams, L. Amerman, A.W. Borgland, A.B. Breon, D.N. Brown, J. Button-Shafer, A.R. Clark, S. Dardin, C. Day, S.F. Dow, Q. Fan, I. Gaponenko, M.S. Gill, F.R. Goozen, S.J. Gowdy, A. Gritsan, Y. Groysman, C. Hernikl, R.G. Jacobsen, R.C. Jared, R.W. Kadel, J. Kadyk, A. Karcher, L.T. Kerth, I. Kipnis, S. Kluth, J.F. Kral, R. Lafever, C. LeClerc, M.E. Levi, S.A. Lewis, C. Lionberger, T. Liu, M. Long, L. Luo, G. Lynch, P. Luft, E. Mandelli, M. Marino, K. Marks, C. Matuk, A.B. Meyer, R. Minor, A. Mokhtarani, M. Momayezi, M. Nyman, P.J. Oddone, J. Ohnemus, D. Oshatz, S. Patton, M. Pedrali-Noy, A. Perazzo, C. Peters, W. Pope, M. Pripstein, D.R. Quarrie, J.E. Rasson, N.A. Roe, A. Romosan, M.T. Ronan, V.G. Shelkov, R. Stone, P.D. Strother, ${ }^{1}$ A.V. Telnov, H. von der Lippe, T.F. Weber, W.A. Wenzel, G. Zizka

Lawrence Berkeley National Laboratory and University of California, Berkeley, CA 94720, USA

P.G. Bright-Thomas, C.M. Hawkes, A. Kirk, D. J. Knowles, S.W. O’Neale, A.T. Watson, N.K. Watson University of Birmingham, Birmingham, B15 2TT, UK

T. Deppermann, H. Koch, J. Krug, M. Kunze, B. Lewandowski, K. Peters, H. Schmuecker, M. Steinke Ruhr Universität Bochum, Inst. f. Experimentalphysik 1, D-44780 Bochum, Germany

J.C. Andress, N.R. Barlow, W. Bhimji, N. Chevalier, P.J. Clark, W.N. Cottingham, N. De Groot, N. Dyce, B. Foster, A. Mass, J.D. McFall, D. Wallom, F.F. Wilson

University of Bristol, Bristol BS8 1TL, UK

K. Abe, C. Hearty, J.A. McKenna, D. Thiessen

University of British Columbia, Vancouver, BC, Canada V6T $1 Z 1$

B. Camanzi, T.J. Harrison, ${ }^{2}$ A.K. McKemey, J. Tinslay

Brunel University, Uxbridge, Middlesex UB8 3PH, UK

E.I. Antohin, V.E. Blinov, A.D. Bukin, D.A. Bukin, A.R. Buzykaev, M.S. Dubrovin, V.B. Golubev, V.N. Ivanchenko, G.M. Kolachev, A.A. Korol, E.A. Kravchenko, S.F. Mikhailov, A.P. Onuchin,

A.A. Salnikov, S.I. Serednyakov, Yu.I. Skovpen, V.I. Telnov, A.N. Yushkov

Budker Institute of Nuclear Physics, Novosibirsk 630090, Russia

\footnotetext{
${ }^{1}$ Now at Queen Mary, University of London, London, E1 4NS, UK

${ }^{2}$ Now at University of Birmingham, Birmingham B15 2TT, UK
} 
J. Booth, A.J. Lankford, M. Mandelkern, S. Pier, D.P. Stoker, G. Zioulas

University of California at Irvine, Irvine, CA 92697, USA

A. Ahsan, K. Arisaka, C. Buchanan, S. Chun

University of California at Los Angeles, Los Angeles, CA 90024, USA

R. Faccini, ${ }^{3}$ D.B. MacFarlane, S.A. Prell, Sh. Rahatlou, G. Raven, V. Sharma

University of California at San Diego, La Jolla, CA 92093, USA

S. Burke, D. Callahan, C. Campagnari, B. Dahmes, D. Hale, P.A. Hart, N. Kuznetsova, S. Kyre,

S. L. Levy, O. Long, A. Lu, J. May, J.D. Richman, W. Verkerke, M. Witherell, S. Yellin

University of California at Santa Barbara, Santa Barbara, CA 93106, USA

J. Beringer, J. DeWitt, D.E. Dorfan, A.M. Eisner, A. Frey, A.A. Grillo, M. Grothe, C.A. Heusch, R.P. Johnson, W. Kroeger, W.S. Lockman, T. Pulliam, W. Rowe, H. Sadrozinski, T. Schalk,

R.E. Schmitz, B.A. Schumm, A. Seiden, E.N. Spencer, M. Turri, W. Walkowiak, M. Wilder, D.C. Williams

University of California at Santa Cruz, Santa Cruz, CA 95064, USA

E. Chen, G.P. Dubois-Felsmann, A. Dvoretskii, J.E. Hanson, D.G. Hitlin, Yu.G. Kolomensky, ${ }^{4}$

S. Metzler, J. Oyang, F.C. Porter, A. Ryd, A. Samuel, M. Weaver, S. Yang, R.Y. Zhu

California Institute of Technology, Pasadena, CA 91125, USA

S. Devmal, T.L. Geld, S. Jayatilleke, S.M. Jayatilleke, G. Mancinelli, B.T. Meadows, M.D. Sokoloff

University of Cincinnati, Cincinnati, OH 45221, USA

P. Bloom, B. Broomer, E. Erdos, S. Fahey, W.T. Ford, F. Gaede, W.C. van Hoek, D.R. Johnson, A.K. Michael, U. Nauenberg, A. Olivas, H. Park, P. Rankin, J. Roy, S. Sen, J.G. Smith, D.L. Wagner University of Colorado, Boulder, CO 80309, USA

J. Blouw, J.L. Harton, M. Krishnamurthy, A. Soffer, W.H. Toki, D.W. Warner, R.J. Wilson, J. Zhang Colorado State University, Fort Collins, CO 80523, USA

T. Brandt, J. Brose, G. Dahlinger, M. Dickopp, R.S. Dubitzky, P. Eckstein, H. Futterschneider, M.L. Kocian, R. Krause, R. Müller-Pfefferkorn, K.R. Schubert, R. Schwierz, B. Spaan, L. Wilden

Technische Universität Dresden, D-01062 Dresden, Germany

L. Behr, D. Bernard, G.R. Bonneaud, F. Brochard, J. Cohen-Tanugi, S. Ferrag, G. Fouque, F. Gastaldi, P. Matricon, P. Mora de Freitas, C. Renard, E. Roussot, S. T'Jampens, C. Thiebaux, G. Vasileiadis, M. Verderi

Ecole Polytechnique, F-91128 Palaiseau, France

A. Anjomshoaa, R. Bernet, F. Di Lodovico, F. Muheim, S. Playfer, J.E. Swain

University of Edinburgh, Edinburgh EH9 3JZ, UK

\footnotetext{
${ }^{3}$ Jointly appointed with Università di Roma La Sapienza, Dipartimento di Fisica and INFN, I-00185 Roma, Italy

${ }^{4}$ Now at LBNL and University of California, Berkeley, CA 94720, USA
} 
M. Falbo

Elon College, Elon College, NC 27244, USA

C. Bozzi, S. Dittongo, M. Folegani, L. Piemontese, A..C. Ramusino

Università di Ferrara, Dipartimento di Fisica and INFN, I-44100 Ferrara, Italy

E. Treadwell

Florida A\&M University, Tallahassee, FL 32307, USA

F. Anulli, ${ }^{5}$ R. Baldini-Ferroli, A. Calcaterra, R. de Sangro, D. Falciai, G. Finocchiaro, P. Patteri, I.M. Peruzzi, ${ }^{5}$ M. Piccolo, Y. Xie, A. Zallo

Laboratori Nazionali di Frascati dell'INFN, I-00044 Frascati, Italy

S. Bagnasco, A. Buzzo, R. Contri, G. Crosetti, P. Fabbricatore, S. Farinon, M. Lo Vetere, M. Macri,

S. Minutoli, M.R. Monge, R. Musenich, M. Pallavicini, R. Parodi, S. Passaggio, F.C. Pastore,

C. Patrignani, M.G. Pia, C. Priano, E. Robutti, A. Santroni

Università di Genova, Dipartimento di Fisica and INFN, I-16146 Genova, Italy

R. Bartoldus, T. Dignan, R. Hamilton, U. Mallik

University of Iowa, Iowa City, IA 52242, USA

J. Cochran, H.B. Crawley, P.A. Fischer, J. Lamsa, R. McKay, W.T. Meyer, E.I. Rosenberg

Iowa State University, Ames, IA 50011-3160, USA

J.N. Albert, C. Beigbeder, M. Benkebil, D. Breton, R. Cizeron, S. Du, G. Grosdidier, C. Hast,

A. Höcker, H. M. Lacker, V. LePeltier, A.M. Lutz, S. Plaszczynski, M.H. Schune, S. Trincaz-Duvoid,

K. Truong, A. Valassi, G. Wormser

Laboratoire de l'Accélérateur Linéaire, F-91898 Orsay, France

O. Alford, D. Behne, R.M. Bionta, J. Bowman, V. Brigljević, A. Brooks, V.A. Dacosta, O. Fackler, D. Fujino, M. Harper, D.J. Lange, M. Mugge, T.G. O’Connor, H. Olson, L. Ott, E. Parker, B. Pedrotti, M. Roeben, X. Shi, K. van Bibber, T.J. Wenaus, D.M. Wright, C.R. Wuest, B. Yamamoto

Lawrence Livermore National Laboratory, Livermore, CA 94550, USA

M. Carroll, P. Cooke, J.R. Fry, E. Gabathuler, R. Gamet, M. George, M. Kay, S. McMahon, ${ }^{6}$ A. Muir, D.J. Payne, R.J. Sloane, P. Sutcliffe, C. Touramanis

University of Liverpool, Liverpool L69 3BX, UK

M.L. Aspinwall, D.A. Bowerman, P.D. Dauncey, I. Eschrich, N.J.W. Gunawardane, R. Martin, J.A. Nash, D.R. Price, P.Sanders, D.Smith

University of London, Imperial College, London, SW7 2BW, UK

D.E. Azzopardi, J.J. Back, P. Dixon, P.F. Harrison, D. Newman-Coburn, ${ }^{7}$ R.J.L. Potter, H.W. Shorthouse, M.I. Williams, P.B. Vidal

Queen Mary, University of London, London, E1 4NS, UK

\footnotetext{
${ }^{5}$ Jointly appointed with Univ. di Perugia, I-06100 Perugia, Italy

${ }^{6}$ Now at University of California at Irvine, Irvine, CA 92697, USA

${ }^{7}$ Deceased
} 
G. Cowan, S. George, M.G. Green, A. Kurup, C.E. Marker, P. McGrath, T.R. McMahon, F. Salvatore, I. Scott, G. Vaitsas

University of London, Royal Holloway and Bedford New College, Egham, Surrey TW20 OEX, UK

D. Brown, C. L. Davis, Y. Li, J. Pavlovich

University of Louisville, Louisville, KY 40292, USA

J. Allison, R.J. Barlow, J.T. Boyd, J. Fullwood, F. Jackson, A. Khan, ${ }^{8}$ G.D. Lafferty, N. Savvas, E.T. Simopoulos, R.J. Thompson, J.H. Weatherall University of Manchester, Manchester M13 9PL, UK

R. Bard, C. Dallapiccola, ${ }^{9}$ A. Farbin, A. Jawahery, V. Lillard, J. Olsen, D.A. Roberts, J.R. Schieck University of Maryland, College Park, MD 20742, USA

G. Blaylock, K.T. Flood, S.S. Hertzbach, R. Kofler, C.S. Lin, S. Willocq, J. Wittlin

University of Massachusetts, Amherst, MA 01003, USA

B. Brau, R. Cowan, F. Taylor, R.K. Yamamoto

Massachusetts Institute of Technology, Cambridge, MA 02139, USA

D.I. Britton, R. Fernholz, ${ }^{10}$ M. Houde, M. Milek, P.M. Patel, J. Trischuk

McGill University, Montréal, QC, Canada H3A 2T8

F. Lanni, F. Palombo

Università di Milano, Dipartimento di Fisica and INFN, I-20133 Milano, Italy

J.M. Bauer, M. Booke, L. Cremaldi, R. Kroeger, M. Reep, J. Reidy, D.A. Sanders, D.J. Summers University of Mississippi, University, MS 38677, USA

J. F. Arguin, M. Beaulieu, J. P. Martin, J. Y. Nief, R. Seitz, P. Taras, A. Woch, V. Zacek

Université de Montréal, Lab. René J.A. Lévesque, Montréal, QC, Canada, H3C 3J7

H. Nicholson, C.S. Sutton

Mount Holyoke College, South Hadley, MA 01075, USA

C. Cartero, N. Cavallo, ${ }^{11}$ G. De Nardo, F. Fabozzi, C. Gatto, L. Lista, D. Piccolo, C. Sciacca

Università di Napoli Federico II, Dipartimento di Fisica and INFN, I-80126 Napoli, Italy

N. M. Cason, J. M. LoSecco

University of Notre Dame, Notre Dame, IN 46556, USA

J.R. G. Alsmiller, T.A. Gabriel, T. Handler, J. Heck

Oak Ridge National Laboratory, Oak Ridge, TN 37831, USA

${ }^{8}$ Now at University of Edinburgh, Edinburgh EH9 3JZ, UK

${ }^{9}$ Now at University of Massachusetts, Amherst, MA 01003, USA

${ }^{10}$ Now at Princeton University, Princeton, NJ 08544, USA

${ }^{11}$ Also with Università della Basilicata, I-85100 Portenza, Italy 
M. Iwasaki, N.B. Sinev,

University of Oregon, Eugene, OR 97403, USA

R. Caracciolo, F. Colecchia, F. Dal Corso, F. Galeazzi, M. Marzolla, G. Michelon, M. Morandin, M. Posocco, M. Rotondo, S. Santi, F. Simonetto, R. Stroili, E. Torassa, C. Voci

Università di Padova, Dipartimento di Fisica and INFN, I-35131 Padova, Italy

P. Bailly, M. Benayoun, H. Briand, J. Chauveau, P. David, C. De la Vaissière, L. Del Buono, J.-F. Genat, O. Hamon, Ph. Leruste, F. Le Diberder, H. Lebbolo, J. Lory, L. Martin, F. Martinez-Vidal, ${ }^{12}$ L. Roos, J. Stark, S. Versillé, B. Zhang

Université Paris VI et VII, F-75252 Paris, France

P.F. Manfredi, L. Ratti, V. Re, V. Speziali

Università di Pavia, Dipartimento di Fisica and INFN, I-27100 Pavia, Italy

E.D. Frank, L. Gladney, Q.H. Guo, J.H. Panetta

University of Pennsylvania, Philadelphia, PA 19104, USA

C. Angelini, G. Batignani, S. Bettarini, M. Bondioli, F. Bosi, M. Carpinelli, F. Forti, A. Gaddi,

D. Gagliardi, M.A. Giorgi, A. Lusiani, P. Mammini, M. Morganti, F. Morsani, N. Neri, A. Profeti, E. Paoloni, F. Raffaelli, M. Rama, G. Rizzo, F. Sandrelli, G. Simi, G. Triggiani

Università di Pisa, Scuola Normale Superiore, and INFN, I-56010 Pisa, Italy

M. Haire, D. Judd, K. Paick, L. Turnbull, D. E. Wagoner

Prairie View A\&M University, Prairie View, TX 77446, USA

J. Albert, C. Bula, M.H. Kelsey, C. Lu, K.T. McDonald, V. Miftakov, B. Sands, S.F. Schaffner, A.J.S. Smith, A. Tumanov, E.W. Varnes

Princeton University, Princeton, NJ 08544, USA

F. Bronzini, A. Buccheri, C. Bulfon, G. Cavoto, D. del Re, F. Ferrarotto, F. Ferroni, K. Fratini, E. Lamanna, E. Leonardi, M.A. Mazzoni, S. Morganti, G. Piredda, F. Safai Tehrani, M. Serra, C. Voena Università di Roma La Sapienza, Dipartimento di Fisica and INFN, I-00185 Roma, Italy

R. Waldi

Universität Rostock, D-18051 Rostock, Germany

P.F. Jacques, M. Kalelkar, R.J. Plano

Rutgers University, New Brunswick, NJ 08903, USA

T. Adye, B. Claxton, J. Dowdell, U. Egede, B. Franek, S. Galagedera, N.I. Geddes, G.P. Gopal, J. Kay, ${ }^{13}$ J. Lidbury, S. Madani, S. Metcalfe, ${ }^{13,14}$ G. Markey, ${ }^{13}$ P. Olley, M. Watt, S.M. Xella

Rutherford Appleton Laboratory, Didcot, Oxon., OX11 OQX, UK

\footnotetext{
${ }^{12}$ Now at Università di Pisa, I-56010 Pisa, Italy

${ }^{13}$ At CLRC Daresbury Laboratory, Daresbury, Warrington, Cheshire, WA4 4AD, UK

${ }^{14}$ Now at Stanford Linear Accelerator Center, Stanford, CA 94309, USA
} 
R. Aleksan, P. Besson, ${ }^{7}$ P. Bourgeois, P. Convert, G. De Domenico, A. de Lesquen, S. Emery, A. Gaidot, S. F. Ganzhur, Z. Georgette, L. Gosset, P. Graffin, G. Hamel de Monchenault, S. Hervé, M. Karolak, W. Kozanecki, M. Langer, G.W. London, V. Marques, B. Mayer, P. Micout, J.P. Mols, J.P. Mouly, Y. Penichot, J. Rolquin, B. Serfass, J.C. Toussaint, M. Usseglio, G. Vasseur, C. Yeche, M. Zito

DAPNIA, Commissariat à l'Energie Atomique/Saclay, F-91191 Gif-sur-Yvette, France

N. Copty, M.V. Purohit, F.X. Yumiceva

University of South Carolina, Columbia, SC 29208, USA

I. Adam, A. Adesanya, P.L. Anthony, D. Aston, J. Bartelt, J. Becla, R. Bell, E. Bloom, C.T. Boeheim, A.M. Boyarski, R.F. Boyce, D. Briggs, F. Bulos, W. Burgess, B. Byers, G. Calderini, R. Chestnut, R. Claus, M.R. Convery, R. Coombes, L. Cottrell, D.P. Coupal, D.H. Coward, W.W. Craddock, S. DeBarger, H. DeStaebler, J. Dorfan, M. Doser, W. Dunwoodie, J.E. Dusatko, S. Ecklund, T.H. Fieguth, D.R. Freytag, T. Glanzman, G.L. Godfrey, G. Haller, A. Hanushevsky, J. Harris, A. Hasan, C. Hee, T. Himel, M.E. Huffer, T. Hung, W.R. Innes, C.P. Jessop, H. Kawahara, L. Keller, M.E. King, L. Klaisner, H.J. Krebs, U. Langenegger, W. Langeveld, D.W.G.S. Leith, S.K. Louie, S. Luitz, V. Luth, H.L. Lynch, J. McDonald, G. Manzin, H. Marsiske, T. Mattison, ${ }^{15}$ M. McCulloch, M. McDougald, D. McShurley, S. Menke, R. Messner, S. Metcalfe, M. Morii, ${ }^{16}$ R. Mount, D. R. Muller, D. Nelson, M. Norby, C.P. O’Grady, L. Olavson, J. Olsen, F.G. O'Neill, G. Oxoby, P. Paolucci, ${ }^{17}$

T. Pavel, J. Perl, M. Pertsova, S. Petrak, G. Putallaz, P.E. Raines, B.N. Ratcliff, R. Reif,

S.H. Robertson, L.S. Rochester, A. Roodman, J.J. Russel, L. Sapozhnikov, O.H. Saxton, T. Schietinger,

R.H. Schindler, J. Schwiening, G. Sciolla, ${ }^{18}$ J.T. Seeman, V.V. Serbo, S. Shapiro, K. Skarpass Sr.,

A. Snyder, E. Soderstrom, A. Soha, S.M. Spanier, A. Stahl, P. Stiles, D. Su, M.K. Sullivan, M. Talby, H.A. Tanaka, J. Va'vra, S.R. Wagner, R. Wang, T. Weber, A.J.R. Weinstein, J.L. White, U. Wienands, W.J. Wisniewski, C.C. Young, N. Yu

Stanford Linear Accelerator Center, Stanford, CA 94309, USA

P.R. Burchat, C.H. Cheng, D. Kirkby, T.I. Meyer, C. Roat

Stanford University, Stanford, CA 94305-4060, USA

R. Henderson, N. Khan

TRIUMF, Vancouver, BC, Canada V6T 2A3

S. Berridge, W. Bugg, H. Cohn, E. Hart, A.W. Weidemann

University of Tennessee, Knoxville, TN 37996, USA

T. Benninger, J.M. Izen, I. Kitayama, X.C. Lou, M. Turcotte

University of Texas at Dallas, Richardson, TX 75083, USA

F. Bianchi, M. Bona, F. Daudo, B. Di Girolamo, D. Gamba, P. Grosso, A. Smol, P..P. Trapani, D. Zanin Università di Torino, Dipartimento di Fisica and INFN, I-10125 Torino, Italy

\footnotetext{
${ }^{15}$ Now at University of British Columbia, Vancouver, BC, Canada V6T 1Z1

${ }^{16}$ Now at Harvard University, Cambridge, MA 02138

${ }^{17}$ Now at Università di Napoli Federico II, I-80126 Napoli, Italy

${ }^{18}$ Now at Massachusetts Institute of Technology, Cambridge, MA 02139, USA
} 
L. Bosisio, G. Della Ricca, L. Lanceri, A. Pompili, P. Poropat, M. Prest, I. Rashevskaia, E. Vallazza, G. Vuagnin

Università di Trieste, Dipartimento di Fisica and INFN, I-34127 Trieste, Italy

\section{R.S. Panvini}

Vanderbilt University, Nashville, TN 37235, USA

A. De Silva, ${ }^{19}$ R. Kowalewski, J.M. Roney

University of Victoria, Victoria, BC, Canada V8W $3 P 6$

H.R. Band, E. Charles, S. Dasu, P. Elmer, J.R. Johnson, J. Nielsen, W. Orejudos, Y. Pan, R. Prepost, I.J. Scott, J. Walsh, ${ }^{12}$ S.L. Wu, Z. Yu, H. Zobernig

University of Wisconsin, Madison, WI 53706, USA

T.B. Moore, H. Neal

Yale University, New Haven, CT 06511, USA

$\overline{{ }^{19} \text { Now at TRIUMF, Vancouver, BC, Canada V6T }} 2 \mathrm{~A} 3$ 


\title{
The BABAR Detector
}

\author{
The BABAR Collaboration
}

BABAR, the detector for the SLAC PEP-II asymmetric $e^{+} e^{-} B$ Factory operating at the $\Upsilon(4 S)$ resonance, was designed to allow comprehensive studies of $C P$-violation in $B$-meson decays. Charged particle tracks are measured in a multi-layer silicon vertex tracker surrounded by a cylindrical wire drift chamber. Electromagnetic showers from electrons and photons are detected in an array of CsI crystals located just inside the solenoidal coil of a superconducting magnet. Muons and neutral hadrons are identified by arrays of resistive plate chambers inserted into gaps in the steel flux return of the magnet. Charged hadrons are identified by $d E / d x$ measurements in the tracking detectors and in a ring-imaging Cherenkov detector surrounding the drift chamber. The trigger, data acquisition and data-monitoring systems, VME- and network-based, are controlled by custom-designed online software. Details of the layout and performance of the detector components and their associated electronics and software are presented.

\section{Introduction}

The primary physics goal of the $B A B A R$ experiment is the systematic study of $C P$-violating asymmetries in the decay of neutral $B$ mesons to $C P$ eigenstates. Secondary goals are precision measurements of decays of bottom and charm mesons and of $\tau$ leptons, and searches for rare processes that become accessible with the high luminosity of the PEP-II $B$ Factory [1]. The design of the detector is optimized for $C P$ violation studies, but it is also well suited for these other physics topics. The scientific goals of the BABAR experiment were first presented in the Letter of Intent [2] and the Technical Design Report [3]; detailed physics studies have been documented in the BABAR Physics Book [4] and earlier workshops [5].

The PEP-II $B$ Factory is an asymmetric $e^{+} e^{-}$ collider designed to operate at a luminosity of $3 \times 10^{33} \mathrm{~cm}^{-2} \mathrm{~s}^{-1}$ and above, at a center-ofmass energy of $10.58 \mathrm{GeV}$, the mass of the $\Upsilon(4 S)$ resonance. This resonance decays exclusively to $B^{0} \bar{B}^{0}$ and $B^{+} B^{-}$pairs and thus provides an ideal laboratory for the study of $B$ mesons. In PEPII, the electron beam of $9.0 \mathrm{GeV}$ collides headon with the positron beam of $3.1 \mathrm{GeV}$ resulting in a Lorentz boost to the $\Upsilon(4 S)$ resonance of $\beta \gamma=0.56$. This boost makes it possible to recon- struct the decay vertices of the two $B$ mesons, to determine their relative decay times, and thus to measure the time dependence of their decay rates. The crucial test of $C P$ invariance is a comparison of the time-dependent decay rates for $B^{0}$ and $\bar{B}^{0}$ to a self-conjugate state. For the cleanest experimental test, this requires events in which one $B$ meson decays to a $C P$ eigenstate that is fully reconstructed and the other $B$ meson is tagged as a $B^{0}$ or a $\bar{B}^{0}$ by its decay products: a charged lepton, a charged kaon, or other flavor sensitive features such as a low momentum charged pion from a $D^{*}$ decay.

The very small branching ratios of $B$ mesons to $C P$ eigenstates, typically $10^{-4}$, the need for full reconstruction of final states with two or more charged particles and several $\pi^{0} \mathrm{~s}$, plus the need to tag the second neutral $B$, place stringent requirements on the detector, which should have

- a large and uniform acceptance down to small polar angles relative to the boost direction;

- excellent reconstruction efficiency for charged particles down to $60 \mathrm{MeV} / c$ and for photons to $20 \mathrm{MeV}$;

- very good momentum resolution to separate small signals from background; 


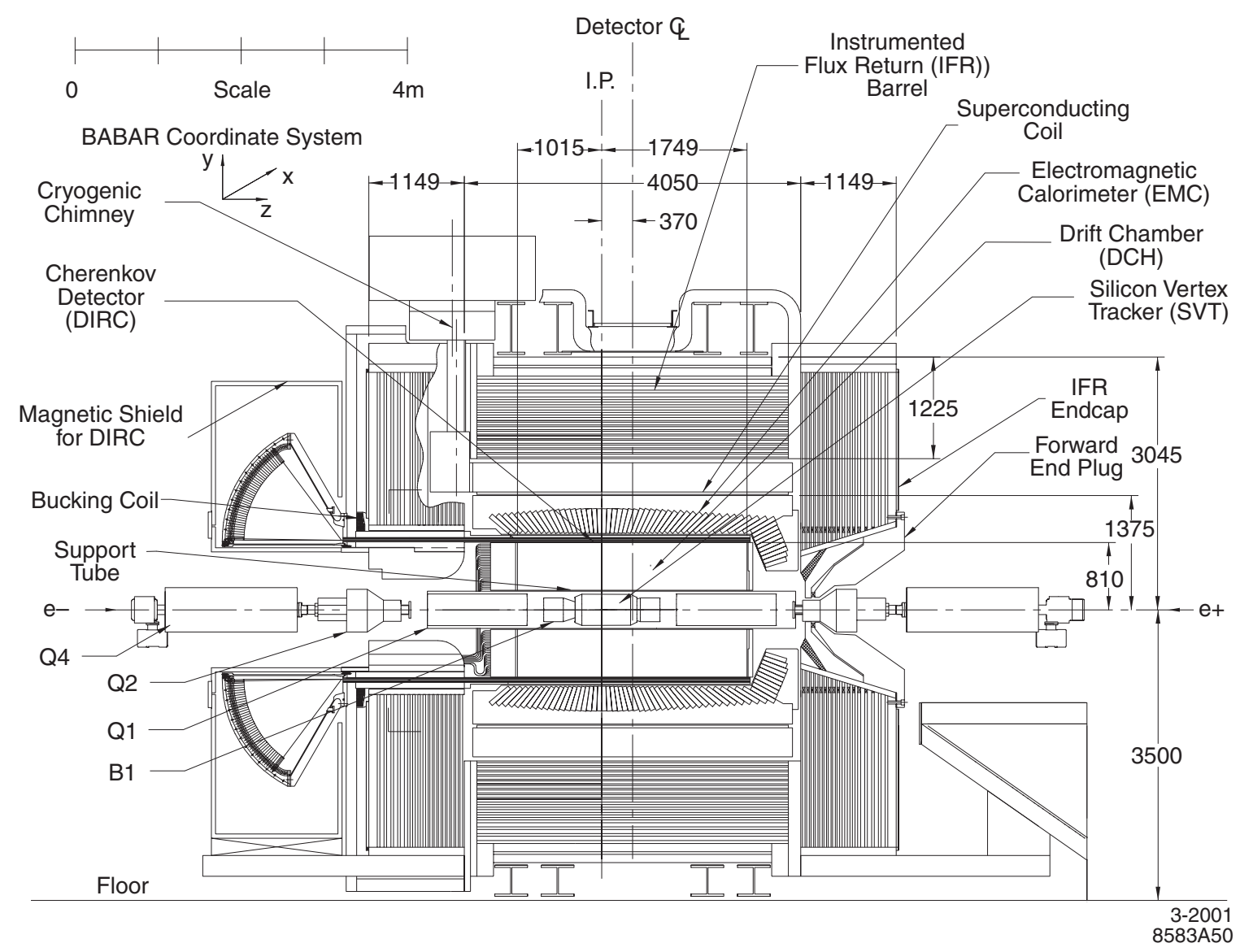

Figure 1. BABAR detector longitudinal section.

- excellent energy and angular resolution for the detection of photons from $\pi^{0}$ and $\eta^{0}$ decays, and from radiative decays in the range from $20 \mathrm{MeV}$ to $4 \mathrm{GeV}$;

- very good vertex resolution, both transverse and parallel to the beam direction;

- efficient electron and muon identification, with low misidentification probablities for hadrons. This feature is crucial for tagging the $B$ flavor, for the reconstruction of charmonium states, and is also important for the study of decays involving leptons;

- efficient and accurate identification of hadrons over a wide range of momenta for
$B$ flavor-tagging, and for the reconstruction of exclusive states; modes such as $B^{0} \rightarrow$ $K^{ \pm} \pi^{\mp}$ or $B^{0} \rightarrow \pi^{+} \pi^{-}$, as well as in charm meson and $\tau$ decays;

- a flexible, redundant, and selective trigger system;

- low-noise electronics and a reliable, high bandwidth data-acquisition and control system;

- detailed monitoring and automated calibration;

- an online computing and network system that can control, process, and store the expected high volume of data; and 


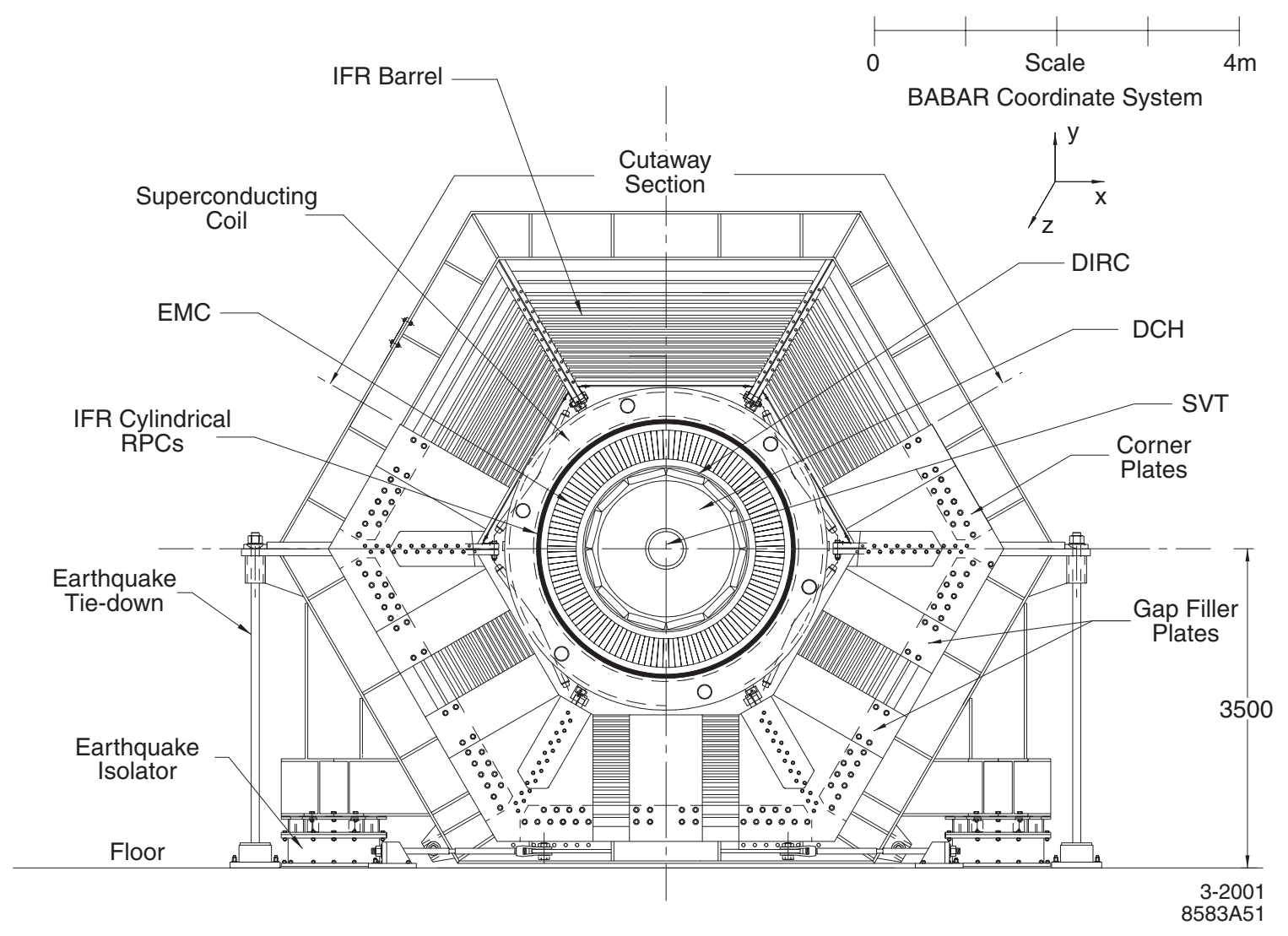

Figure 2. BABAR detector end view.

- detector components that can tolerate significant radiation doses and operate reliably under high-background conditions.

To reach the desired sensitivity for the most interesting measurements, data sets of order $10^{8} \mathrm{~B}$ mesons will be needed. For the peak cross section at the $\Upsilon(4 S)$ of about $1.1 \mathrm{nb}$, this will require an integrated luminosity of order $100 \mathrm{fb}^{-1}$ or three years of reliable and highly efficient operation of a detector with state-of-the art capabilities.

In the following, a brief overview of the principal components of the detector, the trigger, the data-acquisition, and the online computing and control system is given. This overview is followed by brief descriptions of the PEP-II interaction region, the beam characteristics, and of the impact of the beam generated background on the design and operation of the detector. Finally, a detailed presentation of the design, construction, and performance of all BABAR detector systems is provided.

\section{Detector Overview}

The BABAR detector was designed and built by a large international team of scientists and engineers. Details of its original design are documented in the Technical Design Report [3], issued in 1995.

Figure 1 shows a longitudinal section through the detector center, and Figure 2 shows an end view with the principal dimensions. The detector surrounds the PEP-II interaction region. To maximize the geometric acceptance for the boosted $\Upsilon(4 S)$ decays, the whole detector is offset rela- 
tive to the beam-beam interaction point (IP) by $0.37 \mathrm{~m}$ in the direction of the lower energy beam.

The inner detector consists of a silicon vertex tracker, a drift chamber, a ring-imaging Cherenkov detector, and a CsI calorimeter. These detector systems are surrounded by a superconducting solenoid that is designed for a field of 1.5 T. The steel flux return is instrumented for muon and neutral hadron detection. The polar angle coverage extends to $350 \mathrm{mrad}$ in the forward direction and $400 \mathrm{mrad}$ in the backward direction, defined relative to the high energy beam. As indicated in the two drawings, the right handed coordinate system is anchored on the main tracking system, the drift chamber, with the $z$-axis coinciding with its principal axis. This axis is offset relative to the beam axis by about $20 \mathrm{mrad}$ in the horizontal plane. The positive $y$-axis points upward and the positive $x$-axis points away from the center of the PEP-II storage rings.

The detector is of compact design, its transverse dimension being constrained by the $3.5 \mathrm{~m}$ elevation of the beam above the floor. The solenoid radius was chosen by balancing the physics requirements and performance of the drift chamber and calorimeter against the total detector cost. As in many similar systems, the calorimeter was the most expensive single system and thus considerable effort was made to minimize its total volume without undue impact on the performance of either the tracking system or the calorimeter itself. The forward and backward acceptance of the tracking system are constrained by components of PEP-II, a pair of dipole magnets (B1) followed by a pair of quadrupole magnets (Q1). The vertex detector and these magnets are placed inside a support tube $(4.5 \mathrm{~m}$ long and $0.217 \mathrm{~m}$ inner diameter) that is cantilevered from beamline supports. The central section of this tube is fabricated from a carbon-fiber composite.

Since the average momentum of charged particles produced in $B$-meson decay is less than $1 \mathrm{GeV} / c$, the precision of the measured track parameters is heavily influenced by multiple Coulomb scattering. Similarly, the detection efficiency and energy resolution of low energy photons are severely impacted by material in front of the calorimeter. Thus, special care has been

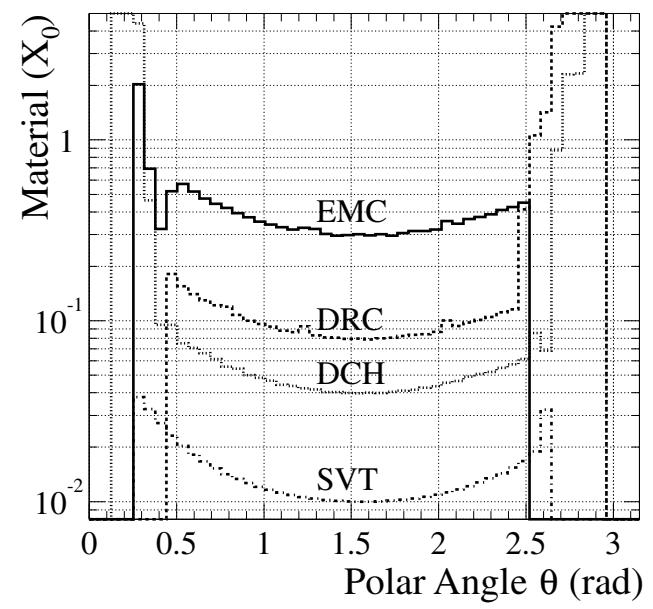

Figure 3. Amount of material (in units of radiation lengths) which a high energy particle, originating from the center of the coordinate system at a polar angle $\theta$, traverses before it reaches the first active element of a specific detector system.

taken to keep material in the active volume of the detector to a minimum. Figure 3 shows the distribution of material in the various detector systems in units of radiation lengths. Each curve indicates the material that a high energy particle traverses before it reaches the first active element of a specific detector system.

\subsection{Detector Components}

An overview of the coverage, the segmentation, and performance of the BABAR detector systems is presented in Table 1.

The charged particle tracking system is made of two components, the silicon vertex tracker (SVT) and the drift chamber (DCH).

The SVT has been designed to measure angles and positions of charged particles just outside the beam pipe. The SVT is composed of five layers of double-sided silicon strip detectors that are assembled from modules with readout at each end, thus reducing the inactive material in the acceptance volume. The inner three layers primarily provide position and angle information for the measurement of the vertex position. They are mounted as close to the water-cooled beryllium beam pipe as practical, thus minimizing the im- 
pact of multiple scattering in the beam pipe on the extrapolation to the vertex. The outer two layers are at much larger radii, providing the coordinate and angle measurements needed for linking SVT and DCH tracks.

The principal purpose of the DCH is the momentum measurement for charged particles. It also supplies information for the charged particle trigger and a measurement of $d E / d x$ for particle identification. The DCH is of compact design, with 40 layers of small, approximately hexagonal cells. Longitudinal information is derived from wires placed at small angles to the principal axis. By choosing low-mass wires, and a helium-based gas mixture the multiple scattering inside the $\mathrm{DCH}$ is minimized. The readout electronics are mounted on the backward endplate of the chamber, minimizing the amount of material in front of the calorimeter endcap.

The DIRC, the detector of internally reflected Cherenkov light, is a novel device providing separation of pions and kaons from about $500 \mathrm{MeV} / \mathrm{c}$ to the kinematic limit of $4.5 \mathrm{GeV} / c$. Cherenkov light is produced in $4.9 \mathrm{~m}$ long bars of synthetic fused silica of rectangular cross section, $1.7 \mathrm{~cm} \times$ $3.5 \mathrm{~cm}$, and transported by total internal reflection, preserving the angle of emission, to an array of photomultiplier tubes. This array forms the backward wall of a toroidal water tank that is located beyond the backward end of the magnet. Images of the Cherenkov rings are reconstructed from the position and time of arrival of the signals in the photomultiplier tubes.

The electromagnetic calorimeter (EMC) is designed to detect electromagnetic showers with excellent energy and angular resolution over the energy range from $20 \mathrm{MeV}$ to $4 \mathrm{GeV}$. This coverage allows the detection of low energy $\pi^{0} \mathrm{~s}$ and $\eta^{0} \mathrm{~s}$ from $B$ decays and higher energy photons and electrons from electromagnetic, weak, and radiative processes. The EMC is a finely segmented array of projective geometry made of thallium doped cesium iodide $(\mathrm{CsI}(\mathrm{Tl}))$ crystals. The crystals are arranged in modules that are supported individually from an external support structure. This structure is built in two sections, a barrel and a forward endcap. To obtain the desired resolution, the amount of material in front of and in- between the crystals is held to a minimum. The individual crystals are read out by pairs of silicon PIN diodes. Low noise analog circuits and frequent, precise calibration of the electronics and energy response over the full dynamic range are crucial for maintaining the desired performance.

The instrumented flux return (IFR) is designed to identify muons and to detect neutral hadrons. For this purpose, the magnet flux return steel in the barrel and the two end doors is segmented into layers, increasing in thickness from $2 \mathrm{~cm}$ on the inside to $10 \mathrm{~cm}$ at the outside. Between these steel absorbers, single gap resistive plate chambers (RPCs) are inserted which detect streamers from ionizing particles via external capacitive readout strips. There are 19 layers of RPCs in the barrel sectors and 18 layers in the end doors. Two additional cylindrical layers of RPCs with four readout planes are placed at a radius just inside the magnet cryostat to detect particles exiting the EMC.

\subsection{Electronics, Trigger, Data Acquisition and Online Computing}

The electronics, trigger, data acquisition, and online computing represent a collection of tightly coupled hardware and software systems. These systems were designed to maximize the physics data acceptance, maintainability, and reliability while managing complexity, and minimizing deadtime, and cost.

- Front-End Electronics (FEE) assemblies, located on the detector, consist of signal processing and digitization electronics along with the data transfer via optical fiber to the data acquisition system.

- A robust and flexible two-level trigger copes with the full beam-beam interaction rate. The first level, Level 1 (L1), is implemented in hardware, the other, Level 3 (L3), in software. Provision is made for an intermediate trigger (Level 2) should severe conditions require additional sophistication.

- The Online Dataflow (ODF), handles digitized data from the FEE through the event building. ODF includes the fast control and timing system (FCTS). 
Table 1

Overview of the coverage, segmentation, and performance of the BABAR detector systems. The notation $(\mathrm{C}),(\mathrm{F})$, and (B) refers to the central barrel, forward and backward components of the system, respectively. The detector coverage in the laboratory frame is specified in terms of the polar angles $\theta_{1}$ (forward) and $\theta_{2}$ (backward). The number of readout channels is listed. The dynamic range (resolution) of the FEE circuits is specified for pulse height (time) measurements by an ADC (TDC) in terms of the number of bits (nsec). Performance numbers are quoted for $1 \mathrm{GeV} / c$ particles, except where noted. The performances for the SVT and DCH are quoted for a combined Kalman fit (for the definition of the track parameters, see Section 7.)

\begin{tabular}{|c|c|c|c|c|c|c|c|}
\hline System & $\begin{array}{c}\theta_{1} \\
\left(\theta_{2}\right)\end{array}$ & $\begin{array}{c}\text { No. } \\
\text { Channels }\end{array}$ & $\begin{array}{l}\mathrm{ADC} \\
\text { (bits) }\end{array}$ & $\begin{array}{c}\text { TDC } \\
(\mathrm{ns})\end{array}$ & $\begin{array}{l}\text { No. } \\
\text { Layers }\end{array}$ & Segmentation & Performance \\
\hline SVT & $\begin{array}{c}20.1^{\circ} \\
\left(-29.8^{\circ}\right) \\
\end{array}$ & $150 \mathrm{~K}$ & 4 & - & 5 & $\begin{array}{c}50-100 \mu m r-\phi \\
100-200 \mu \mathrm{m} \mathrm{z}\end{array}$ & \multirow{2}{*}{$\begin{array}{c}\sigma_{d_{0}}=55 \mu \mathrm{m} \\
\sigma_{z_{0}}=65 \mu \mathrm{m} \\
\sigma_{\phi}=1 \mathrm{mrad} \\
\sigma_{\operatorname{tan\lambda }}=0.001 \\
\sigma_{p_{\mathrm{t}}} / p_{\mathrm{t}}=0.47 \% \\
\sigma(d E / d x)=7.5 \%\end{array}$} \\
\hline $\mathrm{DCH}$ & $\begin{array}{c}17.2^{\circ} \\
\left(-27.4^{\circ}\right)\end{array}$ & 7,104 & 8 & 2 & 40 & $\begin{array}{c}6-8 \mathrm{~mm} \\
\text { drift distance }\end{array}$ & \\
\hline DIRC & $\begin{array}{c}25.5^{\circ} \\
\left(-38.6^{\circ}\right)\end{array}$ & 10,752 & - & 0.5 & 1 & $\begin{array}{c}35 \times 17 \mathrm{~mm}^{2} \\
(r \Delta \phi \times \Delta r) \\
144 \text { bars }\end{array}$ & $\begin{array}{c}\sigma_{\theta_{C}}=2.5 \mathrm{mrad} \\
\text { per track }\end{array}$ \\
\hline $\operatorname{EMC}(\mathrm{C})$ & $\begin{array}{c}27.1^{\circ} \\
\left(-39.2^{\circ}\right)\end{array}$ & $2 \times 5760$ & $17-18$ & - & 1 & $\begin{array}{l}47 \times 47 \mathrm{~mm}^{2} \\
5760 \text { cystals }\end{array}$ & \multirow{2}{*}{$\begin{array}{c}\sigma_{E} / E=3.0 \% \\
\sigma_{\phi}=3.9 \mathrm{mrad} \\
\sigma_{\theta}=3.9 \mathrm{mrad}\end{array}$} \\
\hline $\operatorname{EMC}(\mathrm{F})$ & $\begin{array}{l}15.8^{\circ} \\
\left(27.1^{\circ}\right)\end{array}$ & $2 \times 820$ & & & 1 & 820 crystals & \\
\hline $\operatorname{IFR}(\mathrm{C})$ & $\begin{array}{c}47^{\circ} \\
\left(-57^{\circ}\right)\end{array}$ & $22 \mathrm{~K}+2 \mathrm{~K}$ & 1 & 0.5 & $19+2$ & $20-38 \mathrm{~mm}$ & \multirow{3}{*}{$\begin{array}{c}90 \% \mu^{ \pm} \text {eff. } \\
6-8 \% \pi^{ \pm} \text {mis-id } \\
\text { (loose selection, } \\
1.5-3.0 \mathrm{GeV} / c \text { ) }\end{array}$} \\
\hline $\operatorname{IFR}(\mathrm{F})$ & $\begin{array}{c}20^{\circ} \\
\left(47^{\circ}\right)\end{array}$ & $14.5 \mathrm{~K}$ & & & 18 & $28-38 \mathrm{~mm}$ & \\
\hline $\operatorname{IFR}(B)$ & $\begin{array}{l}-57^{\circ} \\
\left(-26^{\circ}\right)\end{array}$ & $14.5 \mathrm{~K}$ & & & 18 & $28-38 \mathrm{~mm}$ & \\
\hline
\end{tabular}

- A farm of commercial Unix processors and associated software, Online Event Processing (OEP), provides the realtime environment within which complete events are processed by the L3 trigger algorithms, partial event reconstruction is performed for monitoring, and event data are logged to an intermediate storage.

- Software running on a second farm of processors, Online Prompt Reconstruction (OPR), completely reconstructs all physics events, and performs monitoring and con- stants generation in near realtime. Physics event data are transferred to an object database [6] and are made available for further analyses.

- An Online Run Control (ORC) system implements the logic for managing the state of the detector systems, starting and stopping runs, and performing calibrations as well as providing a user control interface.

- A system to control and monitor the detector and its support systems, Online Detec- 
tor Control (ODC), is based upon the Experimental Physics Industrial Control System (EPICS) toolbox [7]. This system includes communication links with PEP-II.

\subsubsection{Electronics}

All BABAR detector systems share a common electronics architecture. Event data from the detector flows through the FEE, while monitoring and control signals are handled by a separate, parallel system. All FEE systems are mounted directly on the detector to optimize performance and to minimize the cable plant, thereby avoiding noise pickup and ground loops in long signal cables. All detector systems utilize standard BABAR interfaces to the data acquisition electronics and software.

Each FEE chain consists of an amplifier, a digitizer, a trigger latency buffer for storing data during the L1 trigger processing, and an event buffer for storing data between the L1 Accept and subsequent transfer to the data acquisition system (see Figure 4). Custom integrated circuits (ICs) have been developed to perform the signal processing. The digital L1 latency buffers function as fixed length data pipelines managed by common protocol signals generated by the FCTS. All de-randomizing event buffers function as FIFOs (first-in-first-out) capable of storing a fixed number of events. During normal operation, analog signal processing, digitization, and data readout occur continuously and simultaneously.

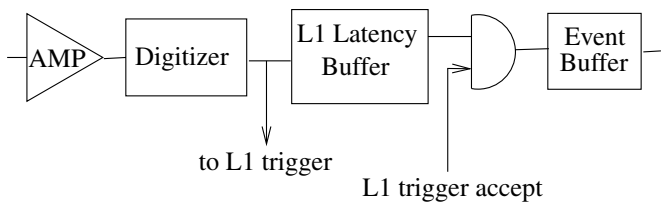

Figure 4. Schematic diagram of the Front-End Electronics (FEE). Analog signals arrive from the left, proceed conditionally through the indicated steps and are injected into the remainder of the data acquisition system.

Since many of the front-end circuits are inaccessible or require significant downtime for access, stringent requirements were placed on reli- ability. Most components underwent comprehensive mean time between failure (MTBF) studies. All circuits underwent a burn-in procedure prior to installation with the goal of minimizing initial failure rates.

\subsubsection{Trigger}

The trigger system operates as a sequence of two independent stages, the second conditional upon the first. The L1 trigger is responsible for interpreting incoming detector signals, recognizing and removing beam-induced background to a level acceptable for the L3 software trigger which runs on a farm of commercial processors.

L1 consists of pipelined hardware processors designed to provide an output trigger rate of $\lesssim 2 \mathrm{kHz}$. The $\mathrm{L} 1$ trigger selection is based on data from DCH, EMC, and IFR. The maximum L1 response latency for a given collision is $12 \mu \mathrm{s}$. Based on both the complete event and L1 trigger information, the L3 software algorithms select events of interest which are then stored for processing. The L3 output rate is administratively limited to $120 \mathrm{~Hz}$ so as not to overload the downstream storage and processing capacity.

$B A B A R$ has no fast counters for triggering purposes, and bunch crossings are nearly continuous at a $4.2 \mathrm{~ns}$ spacing. Dedicated L1 trigger processors receive data continuously clocked in from the DCH, EMC, and IFR detector systems. These processors produce clocked outputs to the fast control system at $30 \mathrm{MHz}$, the time granularity of resultant L1 Accept signals. The arrival of an $L 1$ Accept signal by the data acquisition system causes a portion of each system's L1 latency buffer to be read out, ranging from about $500 \mathrm{~ns}$ for the SVT to $4-16 \mu \mathrm{s}$ for the EMC. Absolute timing information for the event, i.e., associating an event with a particular beam crossing, is determined offline, using DCH track segment timing, waveforms from the EMC, and accelerator timing fiducials.

\subsubsection{Data Acquisition and Online Computing}

The data acquisition and computing systems, responsible for the transport of event data from the detector FEE to mass storage with a min- 
imum of dead time are shown schematically in Figure 5. These systems also interface with the trigger to enable calibrations and testing. Other parts of these systems provide for the control and monitoring of the detector and supporting facilities.

\section{Hardware}

The data acquisition system hardware consists of VME crates, specialized VME-based processors called readout modules (ROMs), the FCTS, a Unix processor farm, various server machines and an Ethernet network. A ROM consists of a Motorola MVME2306 PowerPC single board computer, event buffers, an interface to the FCTS, and a custom Personality Card that connects with the FEE circuits via $1.2 \mathrm{Gbits} / \mathrm{s}$ fiber optic cables. The ROM provides the standard interface between the detector specific FEE, the FCTS, and the event builder. There are 157 ROMs in the system located in 19 physical VME crates divided into 24 logical crates by virtue of segmented backplanes. The FCTS system consists of a VME crate plus individual Fast Control Distribution Modules in each of the data acquisition VME crates. The Unix processor farm consists of 32 Sun workstations.

The detector monitoring and control system consists of a standard set of components, including Motorola MVME177 single-board computers, and other VME modules. With the exception of the solenoid magnet, which has its own control and monitoring, all BABAR detector components use this system.

The online computing system relies on a complex of workstation consoles and servers with 0.8 Tbytes of attached storage, all interconnected with switched $100 \mathrm{Mbits} / \mathrm{s}$ and $1 \mathrm{Gbits} / \mathrm{s}$ Ethernet networks. Multiple 1 Gbits/s Ethernet links connect the experimental hall with the SLAC computing center.

\section{Online Dataflow (ODF)}

The ODF software connects, controls and manages the flow of data in the acquisition hardware with little dead time. This code is divided between embedded processors in the ROMs running the VxWorks [8] realtime operating system and Unix processors running the Solaris operating system. ODF provides configuration and readout of the FEE over fiber links to the ROMs; data transport, buffering, and event building from the ROMs to the Unix farm over a switched $100 \mathrm{Mbits} / \mathrm{s}$ Ethernet network; masking and prescaling of L1 triggers; and logical partitioning of DAQ hardware into multiple, independent data acquisition systems for parallel calibrations and diagnostics. Additional feature extraction (FEX) code in the ROMs extracts physical signals from the raw data, performs gain and pedestal correction, data sparsification, and data formatting. Data from electronics calibrations are accumulated in the ROMs, channel response functions are evaluated, results are compared to reference data and subsequently applied in feature extraction. Calibration data are stored in a conditions database.

\section{Online Event Processing (OEP)}

OEP receives and processes data from the ODF event builder on each of the Unix processors. OEP orchestrates the following tasks: L3 trigger algorithms; fast monitoring to assure data quality; and logging the selected events to disk while merging the multiple data output streams to a single file.

\section{Online Prompt Reconstruction (OPR)}

OPR bridges the online and offline systems [9]. This system reads raw data recorded to disk by OEP and, operating on a farm of 150 Unix processors, selects physics events, performs complete reconstruction, performs rolling calibrations, collects extensive monitoring data for quality assurance, and writes the result into an event store. A rolling calibration is the generation of reconstruction constants during normal event processing, which are then applied to the processing of subsequent data.

\section{Online Detector Control (ODC) and Run Control (ORC)}

The ODC system controls and extensively monitors the electronics, the environment, and 


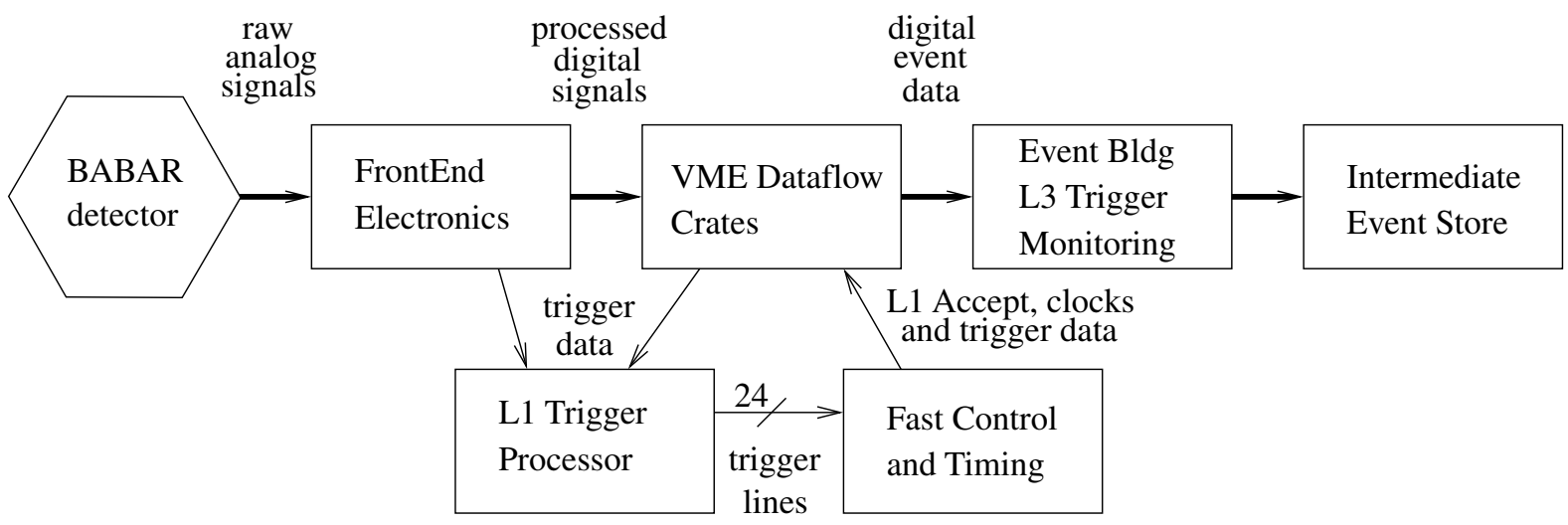

Figure 5. Schematic diagram of the data acquisition.

assures the safety of the detector. Its implementation is based on EPICS, providing detector-wide standardization for control and monitoring, diagnostics and alarm handling. ODC also provides communication with PEP-II and the magnet control system. Monitoring data are archived in an ambient database.

The ORC system ties together the various components of the online system and provides the operator with a single graphical interface to control detector operation. Complex configurations are stored in a configuration database; keys to the configuration used for any run are stored along with the data. The event store, conditions, ambient, and configuration databases are implemented in an object database [6], while other data are stored in a relational database.

\section{The PEP II Storage Rings and Their Impact on the BABAR Detector}

\subsection{PEP-II Storage Rings}

$\mathrm{PEP}-\mathrm{II}$ is an $e^{+} e^{-}$storage ring system designed to operate at a center of mass (c.m.) energy of $10.58 \mathrm{GeV}$, corresponding to the mass of the $\Upsilon(4 S)$ resonance. The parameters of these energy asymmetric storage rings are presented in Table 2. PEP-II has surpassed its design goals, both in terms of the instantaneous and the integrated daily luminosity, with significantly fewer bunches than anticipated. A detailed description of the design and operational experience of PEPII can be found in references [10] and [11].

Table 2

PEP-II beam parameters. Values are given both for the design and for typical colliding beam operation in the first year. HER and LER refer to the high energy $e^{-}$and low energy $e^{+}$ring, respectively. $\sigma_{L x}, \sigma_{L y}$, and $\sigma_{L z}$ refer to the horizontal, vertical, and longitudinal rms size of the luminous region.

\begin{tabular}{lcc}
\hline Parameters & Design & Typical \\
\hline Energy HER/LER $(\mathrm{GeV})$ & $9.0 / 3.1$ & $9.0 / 3.1$ \\
Current HER/LER $(\mathrm{A})$ & $0.75 / 2.15$ & $0.7 / 1.3$ \\
\# of bunches & 1658 & $553-829$ \\
Bunch spacing $(\mathrm{ns})$ & 4.2 & $6.3-10.5$ \\
$\sigma_{L x}(\mu \mathrm{m})$ & 110 & 120 \\
$\sigma_{L y}(\mu \mathrm{m})$ & 3.3 & 5.6 \\
$\sigma_{L z}(\mathrm{~mm})$ & 9 & 9 \\
Luminosity $\left(10^{33} \mathrm{~cm}^{-2} \mathrm{~s}^{-1}\right)$ & 3 & 2.5 \\
Luminosity $\left(\mathrm{pb}^{-1} / \mathrm{d}\right)$ & 135 & 120 \\
\hline
\end{tabular}

PEP-II typically operates on a 40-50 minute fill cycle. At the end of each fill, it takes about three minutes to replenish the beams. After a loss of the stored beams, the beams are refilled in approximately $10-15$ minutes. BABAR divides the data into runs, defined as periods of three hour duration or less during which beam and detector 
conditions are judged to be stable. While most of the data are recorded at the peak of the $\Upsilon(4 S)$ resonance, about $12 \%$ are taken at a c.m. energy $40 \mathrm{MeV}$ lower to allow for studies of non-resonant background.

\subsection{Impact of PEP-II on BABAR Layout}

The high beam currents and the large number of closely-spaced bunches required to produce the high luminosity of PEP-II tightly couple the issues of detector design, interaction region layout, and remediation of machine-induced background. The bunches collide head-on and are separated magnetically in the horizontal plane by a pair of dipole magnets (B1), followed by a series of offset quadrupoles. The tapered B1 dipoles, located at $\pm 21 \mathrm{~cm}$ on either side of the IP, and the Q1 quadrupoles are permanent magnets made of samarium-cobalt placed inside the field of the BABAR solenoid, while the Q2, Q4, and Q5 quadrupoles, located outside or in the fringe field of the solenoid, are standard iron magnets. The collision axis is off-set from the $z$-axis of the $B A B A R$ detector by about $20 \mathrm{mrad}$ in the horizontal plane [12] to minimize the perturbation of the beams by the solenoidal field.

The interaction region is enclosed by a watercooled beam pipe of $27.9 \mathrm{~mm}$ outer radius, composed of two layers of beryllium $(0.83 \mathrm{~mm}$ and $0.53 \mathrm{~mm}$ thick) with a $1.48 \mathrm{~mm}$ water channel between them. To attenuate synchrotron radiation, the inner surface of the pipe is coated with a $4 \mu \mathrm{m}$ thin layer of gold. In addition, the beam pipe is wrapped with $150 \mu \mathrm{m}$ of tantalum foil on either side of the IP, beyond $z=+10.1 \mathrm{~cm}$ and $z=-7.9 \mathrm{~cm}$. The total thickness of the central beam pipe section at normal incidence corresponds to $1.06 \%$ of a radiation length.

The beam pipe, the permanent magnets, and the SVT were assembled and aligned, and then enclosed in a $4.5 \mathrm{~m}$-long support tube which spans the IP. The central section of this tube was fabricated from a carbon-fiber epoxy composite with a thickness of $0.79 \%$ of a radiation length.

\subsection{Monitoring of Beam Parameters}

The beam parameters most critical for BABAR performance are the luminosity, the energies of the two beams, and the position, angles, and size of the luminous region.

\subsubsection{Luminosity}

While PEP-II measures radiative Bhabha scattering to provide a fast monitor of the relative luminosity for operations, BABAR derives the absolute luminosity offline from other QED processes, primarily $e^{+} e^{-}$, and $\mu^{+} \mu^{-}$pairs. The measured rates are consistent and stable as a function of time. For a data sample of $1 \mathrm{fb}^{-1}$, the statistical error is less than $1 \%$. The systematic uncertainty on the relative changes of the luminosity is less than $0.5 \%$, while the systematic error on the absolute value of the luminosity is estimated to be about $1.5 \%$. This error is currently dominated by uncertainties in the Monte Carlo generator and the simulation of the detector. It is expected that with a better understanding of the efficiency, the overall systematic error on the absolute value of the luminosity will be significantly reduced.

\subsubsection{Beam Energies}

During operation, the mean energies of the two beams are calculated from the total magnetic bending strength (including the effects of off-axis quadrupole fields, steering magnets, and wigglers) and the average deviations of the accelerating frequencies from their central values. While the systematic uncertainty in the PEP-II calculation of the absolute beam energies is estimated to be $5-10 \mathrm{MeV}$, the relative energy setting for each beam is accurate and stable to about $1 \mathrm{MeV}$. The rms energy spreads of the LER and HER beams are $2.3 \mathrm{MeV}$ and $5.5 \mathrm{MeV}$, respectively.

To ensure that data are recorded close to the peak of the $\Upsilon(4 S)$ resonance, the observed ratio of $B \bar{B}$ enriched hadronic events to lepton pair production is monitored online. Near the peak of the resonance, a $2.5 \%$ change in the $B \bar{B}$ production rate corresponds to a $2 \mathrm{MeV}$ change in the c.m. energy, a value that is close to the tolerance to which the energy of PEP-II can be held. However, a drop in the $B \bar{B}$ rate does not distinguish between energy settings below or above the $\Upsilon(4 S)$ peak. The sign of the energy change must be determined from other indicators. The best mon- 
itor and absolute calibration of the c.m. energy is derived from the measured c.m. momentum of fully reconstructed $B$ mesons combined with the known $B$-meson mass. An absolute error of $1.1 \mathrm{MeV}$ is obtained for an integrated luminosity of $1 \mathrm{fb}^{-1}$. This error is presently limited by the uncertainty in the $B$-meson mass [13] and by the detector resolution.

The beam energies are necessary input for the calculation of two kinematic variables that are commonly used to separate signal from background in the analysis of exclusive $B$-meson decays. These variables, which make optimum use of the measured quantities and are largely uncorrelated, are Lorentz-invariants which can be evaluated both in the laboratory and c.m. frames.

The first variable, $\Delta E$, can be expressed in Lorentz invariant form as

$\Delta E=\left(2 q_{B} q_{0}-s\right) / 2 \sqrt{s}$,

where $\sqrt{s}=2 E_{\text {beam }}^{*}$ is the total energy of the $e^{+} e^{-}$system in the c.m. frame, and $q_{B}$ and $q_{0}=$ $\left(E_{0}, \vec{p}_{0}\right)$ are the Lorentz vectors representing the momentum of the $B$ candidate and of the $e^{+} e^{-}$ system, $q_{0}=q_{e+}+q_{e-}$. In the c.m. frame, $\Delta E$ takes the familiar form

$\Delta E=E_{B}^{*}-E_{\text {beam }}^{*}$,

here $E_{B}^{*}$ is the reconstructed energy of the $B$ meson. The $\Delta E$ distribution receives a sizable contribution from the beam energy spread, but is generally dominated by detector resolution.

The second variable is the energy-substituted mass, $m_{\mathrm{ES}}$, defined as $m_{\mathrm{ES}}{ }^{2}=q_{B}^{2}$. In the laboratory frame, $m_{\mathrm{ES}}$ can be determined from the measured three-momentum $\vec{p}_{B}$ of the $B$ candidate without explicit knowledge of the masses of the decay products:

$m_{\mathrm{ES}}=\sqrt{\left(s / 2+\vec{p}_{B} \cdot p_{0}\right)^{2} / E_{0}^{2}-p_{B}^{2}}$.

In the c.m. frame $\left(\vec{p}_{0}=0\right)$, this variable takes the familiar form

$m_{\mathrm{ES}}=\sqrt{E_{\text {beam }}^{* 2}-p_{B}^{* 2}}$,

where $p_{B}^{*}$ is the c.m. momentum of the $B$ meson, derived from the momenta of its decay products,

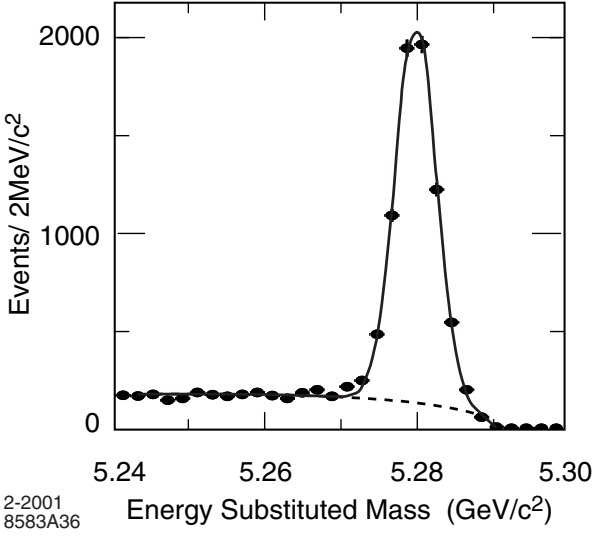

Figure 6. The energy-substituted mass for a sample of 6,700 neutral $B$ mesons reconstructed in the final states $D^{(*)-} \pi^{+}, D^{(*)-} \rho^{+}, D^{(*)-} a_{1}^{+}$, and $J / \psi K^{* 0}$. The background is extrapolated from events outside the signal region.

and the $B$-meson energy is substituted by $E_{\text {beam }}^{*}$. Figure 6 shows the $m_{\mathrm{ES}}$ distribution for a sample of fully reconstructed $B$ mesons. The resolution in $m_{\mathrm{ES}}$ is dominated by the spread in $E_{\text {beam }}^{*}$, $\sigma_{E_{\text {beam }}^{*}}=2.6 \mathrm{MeV}$.

\subsubsection{Beam Direction}

The direction of the beams relative to $B A B A R$ is measured iteratively run-by-run using $e^{+} e^{-} \rightarrow$ $e^{+} e^{-}$and $e^{+} e^{-} \rightarrow \mu^{+} \mu^{-}$events. The resultant uncertainty in the direction of the boost from the laboratory to the c.m. frame, $\vec{\beta}$, is about $1 \mathrm{mrad}$, dominated by alignment errors. This translates into an uncertainty of about $0.3 \mathrm{MeV}$ in $m_{E S} . \vec{\beta}$ is consistent to within $1 \mathrm{mrad}$ with the orientation of the elongated beam spot (see below). It is stable to better than $1 \mathrm{mrad}$ from one run to the next.

\subsubsection{Beam Size and Position}

The size and position of the luminous region are critical parameters for the decay-time-dependent analyses and their values are monitored continuously online and offline. The design values for the size of the luminous region are presented in Table 2. The vertical size is too small to be measured directly. It is inferred from the measured luminosity, the horizontal size, and the beam currents; it varies typically by $1-2 \mu \mathrm{m}$. 
The transverse position, size, and angles of the luminous region relative to the BABAR coordinate system are determined by analyzing the distribution of the distance of closest approach to the $z$-axis of the tracks in well measured two-track events as a function of the azimuth $\phi$. The longitudinal parameters are derived from the longitudinal vertex distribution of the two tracks. A combined fit to nine parameters (three average coordinates, three widths, and three small angles) converges readily, even after significant changes in the beam position. The uncertainties in the average beam position are of the order of a few $\mu \mathrm{m}$ in the transverse plane and $100 \mu \mathrm{m}$ along the collision axis. Run-by-run variations in the beam position are comparable to these measurement uncertainties, indicating that the beams are stable over the period of a typical run. The fit parameters are stored run-by-run in the conditions database. These measurements are also checked offline by measuring the primary vertices in multi-hadron events. The measured horizontal and longitudinal beam sizes, corrected for tracking resolution, are consistent with those measured by PEP-II.

\subsection{Beam Background Sources}

The primary sources of steady-state accelerator backgrounds are, in order of increasing importance: synchrotron radiation in the vicinity of the interaction region; interactions between the beam particles and the residual gas in either ring; and electromagnetic showers generated by beambeam collisions [14-16]. In addition, there are other background sources that fluctuate widely and can lead to very large instantaneous rates, thereby disrupting stable operation.

\subsubsection{Synchrotron Radiation}

Synchrotron radiation in nearby dipoles, the interaction-region quadrupole doublets and the B1 separation dipoles generates many $\mathrm{kW}$ of power and is potentially a severe background. The beam orbits, vacuum-pipe apertures and synchrotron-radiation masks have been designed such that most of these photons are channeled to a distant dump; the remainder are forced to undergo multiple scatters before they can en- ter the BABAR acceptance. The remaining synchrotron radiation background is dominated by $\mathrm{x}$-rays (scattered from tungsten tips of a mask) generated by beam tails in the high-field region of the HER low- $\beta$ quadrupoles. This residual background is relatively low and has not presented significant problems.

\subsubsection{Beam-Gas Scattering}

Beam-gas bremsstrahlung and Coulomb scattering off residual gas molecules cause beam particles to escape the acceptance of the ring if their energy loss or scattering angle are sufficiently large. The intrinsic rate of these processes is proportional to the product of the beam current and the residual pressure (which itself increases with current). Their relative importance, as well as the resulting spatial distribution and absolute rate of lost particles impinging the vacuum pipe in the vicinity of the detector, depend on the beam optical functions, the limiting apertures, and the entire residual-pressure profile around the rings. The separation dipoles bend the energy-degraded particles from the two beams in opposite directions and consequently most BABAR detector systems exhibit occupancy peaks in the horizontal plane, i.e., the LER background near $\phi=0^{\circ}$ and HER background near $\phi=180^{\circ}$.

During the first few months of operation and during the first month after a local venting of the machine, the higher pressures lead to significantly enhanced background from beam-gas scattering. The situation has improved significantly with time due to scrubbing of the vacuum pipe by synchrotron radiation. Towards the end of the first year of data-taking, the dynamic pressure in both rings had dropped below the design goal, and the corresponding background contributions were much reduced. Nevertheless, beam-gas scattering remains the primary source of radiation damage in the SVT and the dominant source of background in all detectors systems, except for the DIRC.

\subsubsection{Luminosity Background}

Radiative Bhabha scattering results in energydegraded electrons or positrons hitting aperture limitations within a few meters of the IP and 
spraying $B A B A R$ with electromagnetic shower debris. This background is directly proportional to the instantaneous luminosity and thus is expected to contribute an increasing fraction of the total background in the future. Already this is the dominant background in the DIRC.

\subsubsection{Background Fluctuations}

In addition to these steady-state background sources, there are instantaneous sources of radiation that fluctuate on diverse time scales:

- beam losses during injection,

- intense bursts of radiation, varying in duration from a few ms to several minutes, currently attributed to very small dust particles, which become trapped in the beam, and

- non-Gaussian tails from beam-beam interactions (especially of the $\mathrm{e}^{+}$beam) that are highly sensitive to adjustments in collimator settings and ring tunes.

These effects typically lead to short periods of high background and have resulted in a large number of BABAR-initiated beam aborts (see below).

\subsection{Radiation Protection and Monitoring}

A system has been developed to monitor the instantaneous and integrated radiation doses, and to either abort the beams or to halt or limit the rate of injection, if conditions become critical. In addition, DCH and IFR currents, as well as DIRC and IFR counting rates, are monitored; abnormally high rates signal critical conditions.

Radiation monitoring and protection systems are installed for the SVT, the DCH electronics, and the EMC. The radiation doses are measured with silicon photodiodes. For the SVT, 12 diodes are arranged in three horizontal planes, at, above, and below the beam level, with four diodes in each plane, placed at $z=+12.1 \mathrm{~cm}$ and $z=-8.5 \mathrm{~cm}$ and at a radial distance of $3 \mathrm{~cm}$ from the beam line [17]. The diode leakage current, after correction for temperature and radiation damage effects, is proportional to the dose rate. The four

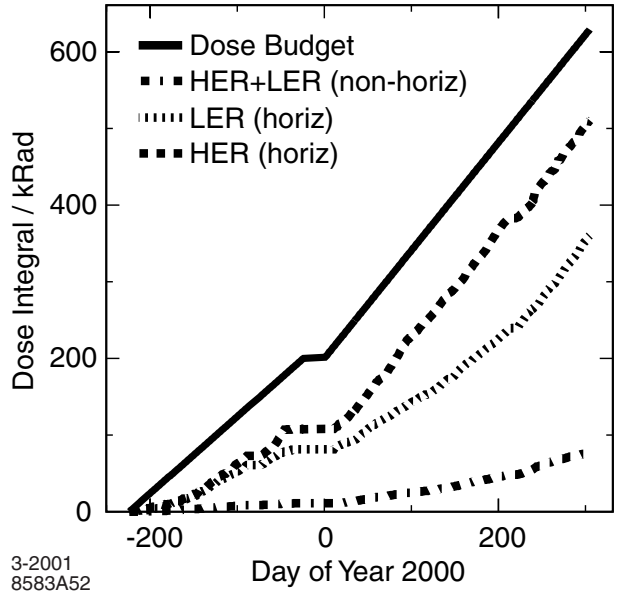

Figure 7 . The integrated radiation dose as measured by PIN diodes located at three different positions, showing contributions from the HER $\left(\phi=180^{\circ}\right)$, and the LER $\left(\phi=0^{\circ}\right)$ in the horizontal plane, and from both beams combined elsewhere. Also shown is the SVT radiation budget for the first 500 days of operation.

diodes in the middle are exposed to about ten times more radiation than the others. These midplane diodes are connected to the beam abort system, while the remaining eight diodes at the top and bottom are used to monitor the radiation dose delivered to the SVT. The accuracy of the measured average dose rate is better than 0.5 $\mathrm{mRad} / \mathrm{s}$. The integrated dose, as measured by the SVT diodes, is presented in Figure 7.

The radiation level at the $\mathrm{DCH}$ and the EMC is more than two orders of magnitude lower than at the SVT. To amplify the signal, the PIN diodes for the DCH and EMC are mounted on small $\mathrm{CsI}(\mathrm{Tl})$ crystals (with a volume of about $10 \mathrm{~cm}^{3}$ ). These silicon diodes are installed in sets of four. Three sets are mounted on the front face of the endcap calorimeter and one set on the backward endplate of the $\mathrm{DCH}$, close to the readout electronics. The signals of the four diodes in each set are summed, amplified, and fed into the radiation protection electronics. Only one of the three diode sets of the EMC is used at any given time. The DCH and the EMC use identical hardware and decision algorithms. They limit injec- 
tion rates whenever an instantaneous dose equivalent to about $1 \mathrm{Rad} /$ day is exceeded.

The SVT employs a different strategy and circuitry to assess whether the measured radiation levels merit a beam abort or a reduction in singlebeam injection rate. Every beam dump initiated by $B A B A R$ is followed by a $10-15$ minute period of injection with significant radiation exposure. Thus, to minimize the ratio of the integrated radiation dose to the integrated luminosity, it has been beneficial to tolerate transient high-dose events as long as the integrated dose remains less than the typical dose accumulated during injection. To differentiate between very high instantaneous radiation and sustained high dose rates, trip thresholds are enforced on two different time scales: an instantaneous dose of $1 \mathrm{Rad}$ accumulated over $1 \mathrm{~ms}$, and an average of $50 \mathrm{mRad} / \mathrm{s}$ measured over a 5 -minute period. During injection, higher thresholds are imposed, since an aborted injection will delay the return to taking data.

Figure 8 shows the daily rate of beam aborts initiated by the SVT protection diodes during the year 2000. Initially, as many as 80 beam aborts were triggered per day, while the average for stable operation was significantly below ten at the end of the run. The measures described above, combined with a significant reduction in large background fluctuations, have been very effective in protecting the detector against radiation damage, as well increasing the combined live time of the machine and detector to greater than $75 \%$.

\subsection{Impact of Beam-Generated Background on BABAR}

Beam-generated backgrounds affect the detector in multiple ways. They cause radiation damage to the detector components and the electronics and thus may limit the lifetime of the experiment. They may also cause electrical breakdown and damage or generate large numbers of extraneous signals leading to problems with bandwidth limitations of the data acquisition system and with event reconstruction. Backgrounds can degrade resolution and decrease efficiency.

The impact of the beam-generated background on the lifetime and on the operation of the dif-

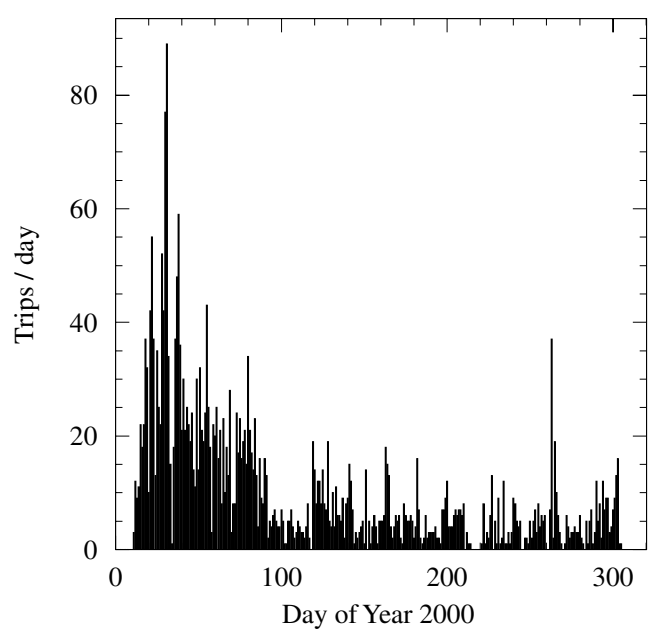

Figure 8. Daily rates of beam aborts initiated by the SVT radiation protection diodes, summed over regular data-taking and PEP-II injection.

ferent detector systems varies significantly. Table 3 lists the limits on the instantaneous and integrated background levels in terms of the total dose and instantaneous observables. These limits are estimates derived from beam tests and experience of earlier experiments. For each detector system, an annual radiation allowance has been established taking into account the total estimated lifetime of the components and the expected annual operating conditions. The typical values accumulated for the first year of operation are also presented in the table.

Systematic studies of background rates were performed with stable stored beams. Measurements of the current-dependence of the backgrounds were carried out for single beams, two beams not colliding, and colliding beams with the goal to identify the principal background sources, to develop schemes of reducing these sources, and to extrapolate to operation at higher luminosity [16]. These experimental studies were complemented by Monte Carlo simulations of beam-gas scattering and of the propagation of showers in the detector. The studies show that the relative importance of the single-beam and luminosity background contributions varies, as illustrated in Figure 9. Data for the IFR are not shown because this system is largely insensitive to beam- 
Table 3

$B A B A R$ background tolerance. Operational limits are expressed either as lifetime limits (radiation-damage and aging-related quantities), or in terms of instantaneous observables (DCH current, DIRC and L1trigger rates).

\begin{tabular}{|c|c|c|c|}
\hline Detector system & $\begin{array}{l}\text { Limiting factor } \\
\text { and impact }\end{array}$ & $\begin{array}{l}\text { Operational } \\
\text { limit }\end{array}$ & $\begin{array}{l}\text { First-year } \\
\text { typical }\end{array}$ \\
\hline $\begin{array}{l}\text { SVT sensors } \\
\text { and electronics }\end{array}$ & $\begin{array}{l}\text { Integrated dose: } \\
\text { radiation damage }\end{array}$ & 2 MRad & $\begin{array}{c}0.33 \text { MRad } \\
\text { (hor.-plane modules) } \\
0.06 \text { MRad } \\
\text { (other modules) }\end{array}$ \\
\hline SVT sensors & $\begin{array}{l}\text { Instantaneous dose: } \\
\text { diode shorts }\end{array}$ & $1 \mathrm{Rad} / \mathrm{ms}$ & $\mathrm{N} / \mathrm{A}$ \\
\hline DCH: electronics & $\begin{array}{l}\text { Integrated dose: } \\
\text { radiation damage }\end{array}$ & $20 \mathrm{kRad}$ & $\leq 100 \operatorname{Rad}$ \\
\hline DCH: wire current & $\begin{array}{c}\text { Accumulated charge: } \\
\text { wire aging }\end{array}$ & $100 \mathrm{mC} / \mathrm{cm}$ & $8 \mathrm{mC} / \mathrm{cm}$ \\
\hline DCH: total current & HV system limitations & $1000 \mu \mathrm{A}$ & $\begin{array}{c}250 \mu \mathrm{A} \\
\text { (steady-state) }\end{array}$ \\
\hline DIRC PMTs & $\begin{array}{l}\text { Counting rate: } \\
\text { TDC deadtime }\end{array}$ & $200 \mathrm{kHz}$ & $\begin{array}{c}110 \mathrm{kHz} \text { (steady-state, } \\
\text { well-shielded sector) }\end{array}$ \\
\hline EMC crystals & $\begin{array}{l}\text { Integrated dose: } \\
\text { radiation damage }\end{array}$ & $10 \mathrm{kRad}$ & $\begin{array}{l}0.25 \mathrm{kRad} \\
\text { (worst case) }\end{array}$ \\
\hline L1 trigger & $\begin{array}{l}\text { Counting rate: } \\
\text { DAQ dead time }\end{array}$ & $2 \mathrm{kHz}$ & $\begin{array}{c}0.7 \mathrm{kHz} \\
\text { (steady-state) }\end{array}$ \\
\hline
\end{tabular}

generated backgrounds, except for the outer layer of the forward endcap, due to insufficient shielding of the external beam line components.

The experience of the first year of operation and the concern for future operation for each of the detectors are summarized as follows.

SVT: The most significant concern for the SVT with regard to machine background is the integrated radiation dose. The instantaneous and integrated dose rates in the radiation protection diodes are representative, to within about a factor of two, of the radiation doses absorbed by the SVT modules. The exposure in the horizontal planes is an order of magnitude larger than elsewhere, averaging $15-25 \mathrm{mRad} / \mathrm{s}$ during stable beam operation. The highest integrated dose is $450 \mathrm{kRad}$, roughly $1 \mathrm{kRad} /$ day. This dose is about $30 \%$ below the allowance, giving some con- fidence that the SVT can sustain operation for several more years (see Figure 7).

DCH: For the DCH, the currents on the wires are the main concern, both because of the limited capacity of the HV power supplies and the effect of wire aging. The currents drawn are approximately uniformly distributed among the 44 HV supplies, one for each quadrant of superlayers $2-10$, and two per quadrant for superlayer 1. Consequently, the total current limit is close to the sum of the limits of the individual supplies. During stable operation the total chamber current is $200-300 \mu \mathrm{A}$. However, radiation spikes can lead to currents that occasionally exceed the limit of $1000 \mu \mathrm{A}$, causing $\mathrm{HV}$ supplies to trip. Other background effects are measured to be well below the estimated lifetime limits and thus are not a serious issue at this time. The average wire 


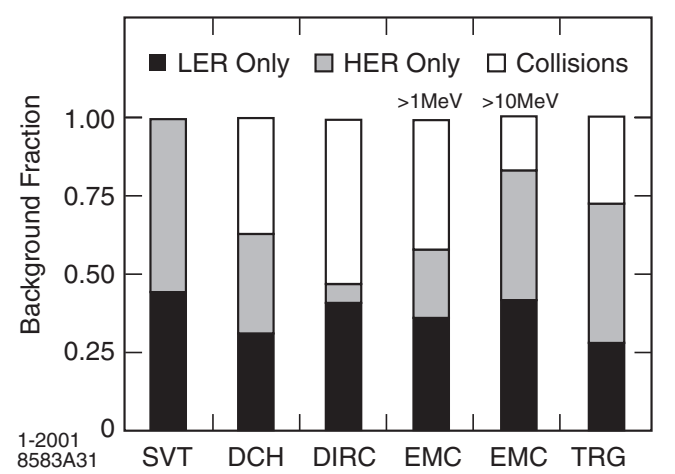

Figure 9. Fractional steady-state background contributions in BABAR detector systems, measured for single beams and colliding beams under stable conditions $\left(I^{+}=1.25 \mathrm{~A}, I^{-}=0.75 \mathrm{~A}\right.$, $\left.L=2.3 \times 10^{33} \mathrm{~cm}^{-2} \mathrm{~s}^{-1}\right)$ in July 2000 . The contributions are derived from the measured doses in the horizontal plane for the SVT, the total currents in the $\mathrm{DCH}$, the rates in the DIRC photomultipliers, the occupancy and number of photons above $10 \mathrm{MeV}$ in the EMC, and the L1 trigger rates.

occupancy has not exceeded 1-2\% during stable operation, but the extrapolation to future operation at higher luminosity and currents remains a major concern.

DIRC: The DIRC radiators, made of synthetic fused silica, were tested up to doses of 100 kRad without showing any measurable effects and thus radiation damage is not a concern. The present operational limit of the DIRC is set by the TDC electronics which induce significant dead time at rates above $250 \mathrm{kHz}$, well above the stable beam rate of $110 \mathrm{kHz}$ in well shielded areas. Roughly half of the present rate is luminosityrelated and can be attributed to radiative Bhabha scattering. The counting rate is due to debris from electromagnetic showers entering the waterfilled stand-off box. Efforts are underway to improve the shielding of the beam pipe nearby.

EMC: The lifetime of the EMC is set by the reduction in light collection in the CsI crystals due to radiation damage. The cumulative dose absorbed by the EMC is measured by a large set of RadFETs placed in front of the barrel and end- cap crystals. RadFETs [18] are realtime integrating dosimeters based on solid-state Metal Oxide Semiconductor (MOS) technology. The absorbed dose increases approximately linearly with the integrated luminosity. The highest dose to date was observed in the innermost ring of the endcap, close to $250 \mathrm{Rad}$, while the barrel crystals accumulated about $80 \mathrm{Rad}$. The observed reduction in light collection of $10-15 \%$ in the worst place, and $4-7 \%$ in the barrel, is consistent with expectation (see Section 9).

The energy resolution is dependent on the single crystal readout threshold, currently set at $1 \mathrm{MeV}$. During stable beam conditions the average crystal occupancy for random triggers is $16 \%$, with $10 \%$ originating from electronics noise in the absence of any energy deposition. The spectrum of photons observed in the EMC from the LER and HER is presented in Figure 10. The HER produces a somewhat harder spectrum. The average occupancy for a threshold of $1 \mathrm{MeV}$ and the average number of crystals with a deposited energy of more than $10 \mathrm{MeV}$ are shown in Figure 11 as a function of beam currents for both single and colliding beams. The occupancy increases significantly at smaller polar angles, in the forward endcap and the backward barrel sections, and in the horizontal plane. The rate increase is approximately linear with the single beam currents. Background rates recorded with separated beams are consistent with those produced by single beams. For colliding beams, there is an additional flux of photons originating from small angle radiative Bhabha scattering. This effect is larger for low energy photons and thus it is expected that at higher luminosities the low energy background will raise the occupancy and thereby limit the EMC energy resolution.

L1 Trigger: During stable beam operation, the typical $\mathrm{L} 1$ trigger rate is below $1 \mathrm{kHz}$, more than a factor of two below the data acquisition bandwidth limit of about $2.0-2.5 \mathrm{kHz}$. Experience shows that background bursts and other rate spikes can raise the data volume by as much as a factor of two and thus it is necessary to aim for steady state rates significantly below the stated limit. For the L1 trigger, the dominant sources of DCH triggers are particles generated by inter- 


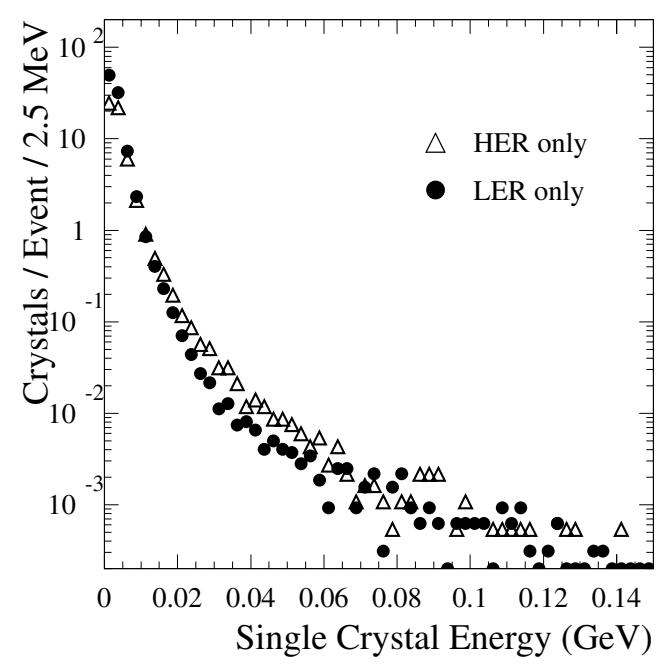

Figure 10. The energy spectrum of photons recorded in the EMC by random triggers with single beams at typical operating currents, LER at $1.1 \mathrm{~A}$ and HER at $0.7 \mathrm{~A}$. The electronic noise has been subtracted.

actions in vacuum flanges and the B1 magnets (see Figure 86 in Section 11). This effect is most pronounced in the horizontal plane. At present, the HER background is twice as high as that of the LER, and the colliding beams contribute less than half of the combined LER and HER single beam triggers.

\subsection{Summary and Outlook}

Towards the end of the first year of data-taking, PEP-II routinely delivered beams close to design luminosity. Due to the very close cooperation with the PEP-II operations team, the machine-induced backgrounds have not been a major problem once stable conditions were established. The background monitoring and protection system has become a reliable and useful tool to safeguard the detector operation.

Currently planned upgrades are expected to raise the luminosity to $1.5 \times 10^{34} \mathrm{~cm}^{-2} \mathrm{~s}^{-1}$ within a few years. The single beam backgrounds will increase with beam currents and the luminosity background is projected to exceed, or at best remain comparable to, the beam-gas contribution. Measures are being prepared to reduce

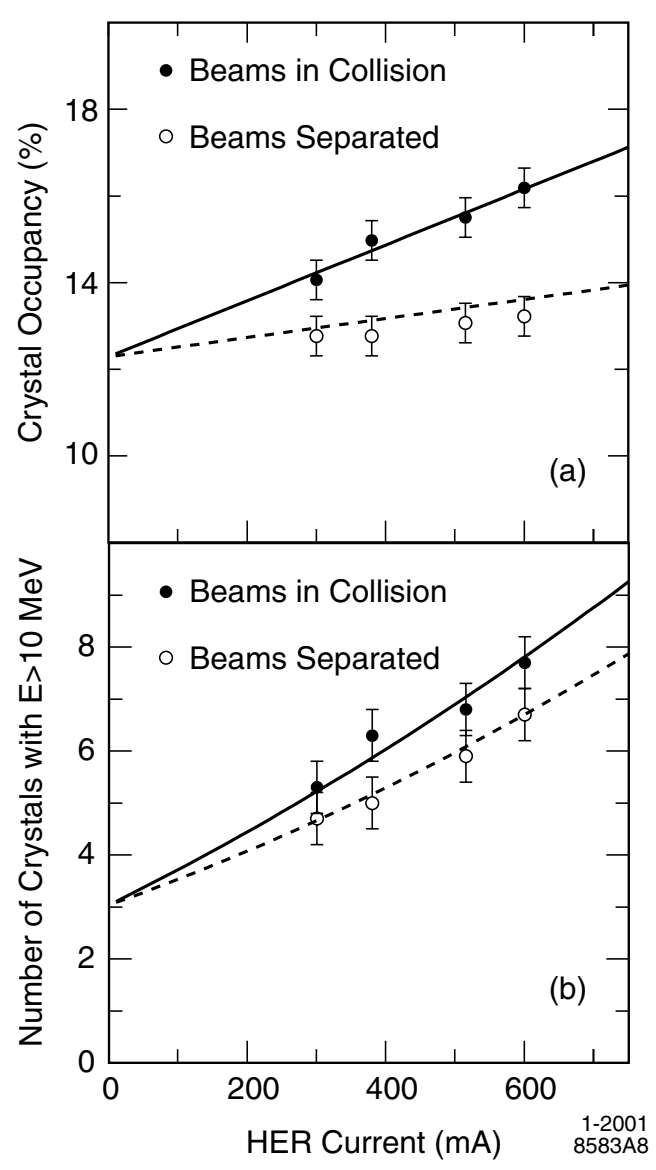

Figure 11. Average rates in the EMC for random triggers as a function of the HER current for a fixed LER current of 1.1A, both for separated and colliding beams; a) the single crystal occupancy for thresholds of $1 \mathrm{MeV}$ and b) the number of crystals with a deposited energy greater than $10 \mathrm{MeV}$. The solid curves represent a fit to the colliding beam data, the dashed curves indicate the sum of rates recorded for single beams.

the sources and the impact of machine-related background on BABAR, among them upgrades to the DCH power supply system and to the DIRC TDC electronics, the addition of localized shielding against shower debris (especially for the DIRC stand-off box), new vacuum chambers, adjustable collimators, and additional pumping capacity in critical regions upbeam of the interaction point.

With the expected increase in LER current 


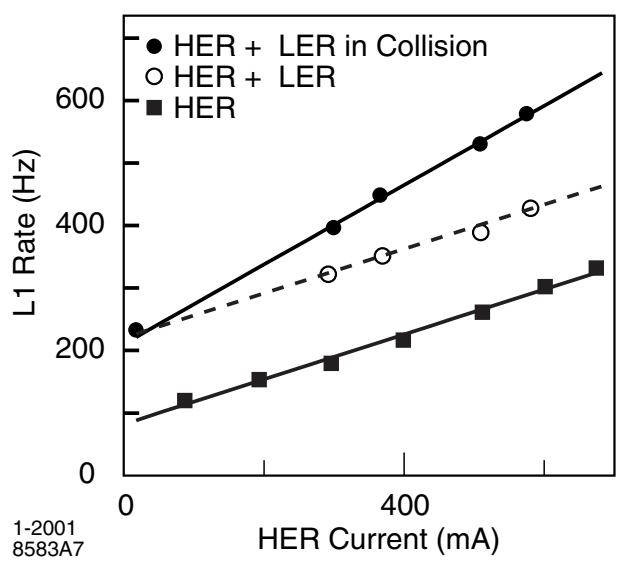

Figure 12. The L1 trigger rate as a function of the HER current for single beam only, for both beams, separated and colliding (with a LER current of $1.1 \mathrm{~A})$.

and in luminosity, both the single-beam and the luminosity-generated L1 trigger rates will increase and are projected to exceed $2 \mathrm{kHz}$ (see Figure 12). Therefore, the DCH trigger is being upgraded to improve the rejection of background tracks originating from outside the luminous region. In addition, the data acquisition and data processing capacity will need to be expanded to meet the demands of higher luminosity.

Overall, the occupancy in all systems, except the IFR, will probably reach levels that are likely to impact the resolution and reconstruction efficiency. For instance, the occupancy in the EMC is expected to more than double. Thus, beyond the relatively straight forward measures currently planned for BABAR system upgrades, detailed studies of the impact of higher occupancy will be necessary for all systems.

\section{The Solenoid Magnet and Flux Return}

\subsection{Overview}

The BABAR magnet system consists of a superconducting solenoid [19], a segmented flux return and a field compensating or bucking coil. This system provides the magnetic field which enables charged particle momentum measurement, serves as the hadron absorber for hadron/muon separation, and provides the overall structure and sup- port for the detector components. Figures 1 and 2 show key components of the BABAR magnet system and some of the nearby PEP-II magnets.

The magnet coil cryostat is mounted inside the hexagonal barrel flux return by four brackets on each end. The flux return end doors are each split vertically and mounted on skids that rest on the floor. To permit access to the inner detector, the doors can be raised and moved on rollers. At the interface between the barrel and the end doors, approximately $60 \%$ of the area is occupied by structural steel and filler plates; the remaining space is reserved for cables and utilities from the inner detectors. A vertical, triangular chase cut into the backward end doors contains the cryostat chimney. Table 4 lists the principal parameters of the magnet system. The total weight of the flux return is approximately 870 metric tons.

To optimize the detector acceptance for unequal beam energies, the center of the BABAR detector is offset by $370 \mathrm{~mm}$ in the electron beam direction. The principal component of the magnetic field, $B_{z}$, lies along the $+z$ axis; this is also the approximate direction of the electron beam. The backward end door is tailored to accommodate the DIRC bar boxes and to allow access to the drift chamber electronics. Both ends allow space and adequate shielding for the PEP-II quadrupoles.

\subsection{Magnetic Field Requirements and Design}

\subsubsection{Field Requirements}

A solenoid magnetic field of $1.5 \mathrm{~T}$ was specified in order to achieve the desired momentum resolution for charged particles. To simplify track finding and fast and accurate track fitting, the magnitude of the magnetic field within the tracking volume was required to be constant within a few percent.

The magnet was designed to minimize disturbance of operation of the PEP-II beam elements. The samarium-cobalt B1 dipole and Q1 quadrupole magnets are located inside the solenoid as shown in Figure 1. Although these magnets can sustain the high longitudinal field of $1.5 \mathrm{~T}$, they cannot tolerate a large radial component. Specifically, the field cannot exceed $0.25 \mathrm{~T}$ 
Table 4

Magnet Parameters

\begin{tabular}{|c|c|c|}
\hline Field Parameters & & \\
\hline Central Field & 1.5 & $\mathrm{~T}$ \\
\hline $\begin{array}{l}\text { Max. Radial Field } \\
\text { at Q1 and } r=200 \mathrm{~mm}\end{array}$ & $<0.25$ & $\mathrm{~T}$ \\
\hline Leakage into PEP-II & $<0.01$ & $\mathrm{~T}$ \\
\hline Stored Energy & 27 & MJ \\
\hline \multicolumn{3}{|l|}{ Steel Parameters } \\
\hline Overall Barrel Length & 4050 & $\mathrm{~mm}$ \\
\hline $\begin{array}{l}\text { Overall Door Thickness } \\
\text { (incl. gaps for RPCs) }\end{array}$ & 1149 & $\mathrm{~mm}$ \\
\hline Overall Height & 6545 & $\mathrm{~mm}$ \\
\hline Plates in Barrel & 18 & \\
\hline 9 & 20 & $\mathrm{~mm}$ \\
\hline 4 & 30 & $\mathrm{~mm}$ \\
\hline 3 & 50 & $\mathrm{~mm}$ \\
\hline 2 & 100 & $\mathrm{~mm}$ \\
\hline Plates in Each Door & 18 & \\
\hline 9 & 20 & $\mathrm{~mm}$ \\
\hline 4 & 30 & $\mathrm{~mm}$ \\
\hline 4 & 50 & $\mathrm{~mm}$ \\
\hline 1 & 100 & $\mathrm{~mm}$ \\
\hline \multicolumn{3}{|l|}{ Main Coil Parameters } \\
\hline $\begin{array}{l}\text { Mean Diameter of } \\
\text { Current Sheet }\end{array}$ & 3060 & $\mathrm{~mm}$ \\
\hline Current Sheet Length & 3513 & $\mathrm{~mm}$ \\
\hline Number of layers & 2 & \\
\hline Operating Current & 4596 & A \\
\hline $\begin{array}{l}\text { Conductor Current } \\
\text { Density }\end{array}$ & 1.2 & $\mathrm{kA} / \mathrm{mm}^{2}$ \\
\hline Inductance & 2.57 & $\mathrm{H}$ \\
\hline \multicolumn{3}{|l|}{ Bucking Coil Parameters } \\
\hline Inner Diameter & 1906 & $\mathrm{~mm}$ \\
\hline Operating Current & 200 & A \\
\hline Number of Turns & 140 & \\
\hline \multicolumn{3}{|l|}{ Cryostat Parameters } \\
\hline Inner Diameter & 1420 & $\mathrm{~mm}$ \\
\hline Radial Thickness & 350 & $\mathrm{~mm}$ \\
\hline Total Length & 3850 & $\mathrm{~mm}$ \\
\hline Total Material (Al) & $\sim 126$ & $\mathrm{~mm}$ \\
\hline
\end{tabular}

at a radius $r=200 \mathrm{~mm}$ (assuming a linear dependence of $B_{r}$ on $r$ ) without degrading their field properties due to partial demagnetization. The conventional iron quadrupoles Q2, Q4, and Q5 are exposed to the solenoid stray fields. To avoid excessive induced skew octupole components, the stray field leaking into these beam elements is required to be less than $0.01 \mathrm{~T}$ averaged over their apertures.

\subsubsection{Field Design Considerations}

Saturation of the steel near the coil and the gap between the coil and the steel leads to field nonuniformities. To control these non-uniformities, the current density of the coil is increased at the ends relative to the center by reducing the thickness of the aluminum stabilizer. While the requirements on the radial field component at Q1 inside the solenoid can be satisfied easily at the forward end, the shape of the backward plug had to be specifically designed to simultaneously control field uniformity and unwanted radial components.

Leakage of magnetic flux is a problem, in particular at the backward end. A bucking coil, mounted at the face of the backward door and surrounding the DIRC strong support tube, is designed to reduce the stray field to an acceptable level for the DIRC photomultipliers and the PEP-II quadrupoles.

\subsubsection{Magnetic Modeling}

Extensive calculations of the magnetic field were performed to develop the detailed design of the flux return, the solenoid coil, and the bucking coil. To crosscheck the results of these calculations the fields were modeled in detail in two and three dimensions using commercial software [20]. The shape of the hole in each end door was designed by optimizing various parameters, such as the minimum steel thickness in areas of saturation. The design of the hole in the forward door was particularly delicate because the highly saturated steel is very close to the Q2 quadrupole. The multiple finger design of the hole was chosen to control the saturation of the steel.

Most of the design work was performed in two dimensions, but some three dimensional calcula- 
tions were necessary to assure the accuracy of modeling the transitions between the end doors and the barrel, the leakage of field into the PEPII magnets, and the impact of that leakage on the multipole purity $[21,22]$. The computations of the leakage field were done for central field of $1.7 \mathrm{~T}$ instead of $1.5 \mathrm{~T}$ to provide some insurance against uncertainties in the modeling of complex steel shapes and the possible variations of the magnetic properties of the steel.

\subsection{Steel Flux Return \\ 4.3.1. Mechanical and Magnetic Forces}

The magnet flux return supports the detector components on the inside, but this load is not a major issue. Far greater demands are placed on the structural design by the magnetic forces and the mechanical forces from a potential earthquake.

Magnetic forces are of three kinds. First, there is a symmetric magnetic force on the end doors which was taken into consideration in their design and construction. Second, there is an axial force on the solenoid due to the forwardbackward asymmetry of the steel. Because the steel is highly saturated in places, the magnitude of the field asymmetry changes when the current is raised from zero, and there is no position of the solenoid at which the force remains zero at all currents. Because it is important that this axial force should not change sign, which could cause a quench, the superconducting solenoid was deliberately offset by $30 \mathrm{~mm}$ towards the forward door. This offset was chosen to accommodate a worst case scenario, including uncertainties in the calculation. Third, during a quench of the superconducting coil, eddy currents in conducting components inside the magnetic volume could generate sizable forces. These forces were analyzed for components such as the endplates of the drift chamber and the electromagnetic calorimeter and were found not to be a problem.

\subsubsection{Earthquake Considerations}

Because SLAC is located in an earthquake zone, considerable attention has been given to protecting the detector against severe damage from such an event. The entire detector is supported on four earthquake isolators, one at each corner, which limit the component acceleration in the horizontal plane to $0.4 \mathrm{~g}$. However, these isolators offer no protection in the vertical direction. Vertical ground accelerations of $0.6 \mathrm{~g}$ are considered credible and actual component accelerations may be considerably larger due to resonances. By taking into account resonant frequencies and the expected frequency spectra of earthquakes, the magnet and all detector components have been designed to survive these accelerations without serious damage. Because the magnet is isolated from the ground moving beneath it, worst case clearances to external components, e.g.,PEP-II components, are provided. It is expected that even during a major earthquake, damage would be modest.

\subsubsection{Fabrication}

The flux return was fabricated [23] from drawings prepared by the BABAR engineering team. A primary concern was the magnetic properties of the steel. The need for a high saturation field dictated the choice of a low carbon steel, specified by its chemical content (close to AISI 1006). The manufacturer supplied sample steel for critical magnetic measurements and approval. The availability of very large tools at the factory made it possible to machine the entire face of each end of the assembled barrel, thus assuring a good fit of the end doors. The entire flux return was assembled at the factory, measured mechanically, and inspected before disassembly for shipment.

\subsection{Magnet Coils}

The design of the superconducting solenoid is conservative and follows previous experience. The superconducting material is composed of niobium-titanium ( $46.5 \%$ by weight $\mathrm{Nb}$ ) filaments, each less than $40 \mu \mathrm{m}$ in diameter. These filaments are then wound into $0.8 \mathrm{~mm}$ strands, 16 of which are then formed into Rutherford cable measuring $1.4 \times 6.4 \mathrm{~mm}$. The final conductor [24] consists of Rutherford cable coextruded with pure aluminum stabilizer measuring $4.93 \times 20.0 \mathrm{~mm}$ for use on the outer, high current density portion of the solenoid, and 


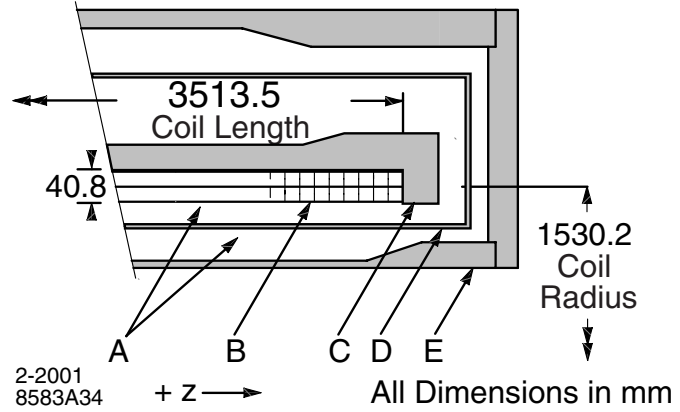

Figure 13. A portion of cryostat assembly. The forward end is shown. Legend: (A) evacuated spaces filled with IR-reflective insulator; (B) superconducting coil (2-layers); (C) aluminum support cylinder; (D) aluminum heat shield; (E) aluminum cryostat housing.

$8.49 \times 20.0 \mathrm{~mm}$ for the central, lower current density portion. The conductor is covered in an insulating dry wrap fiberglass cloth which is vacuum impregnated with epoxy. The conductor has a total length of $10.3 \mathrm{~km}$.

The solenoid is indirectly cooled to an operating point of $4.5 \mathrm{~K}$ using a thermo-syphon technique. Liquid helium [25] is circulated in channels welded to the solenoid support cylinder. Liquid helium and cold gas circulate between the solenoid, its shields, the liquefier-refrigerator and a $4000 \ell$ storage dewar via $60 \mathrm{~m}$ of coaxial, gasscreened, flexible transfer line. The solenoid coil and its cryostat were fabricated [26] to drawings prepared by the BABAR engineering team. Before shipment [27], the fully assembled solenoid was cooled to operating temperature and tested with currents of up to $1000 \mathrm{~A}$, limited by coil forces in the absence of the iron flux return.

A portion of the cryostat assembly, containing the solenoid coil, its support cylinder and heat shield, is shown in Figure 13.

To reduce the leakage fields into the PEP-II components and the DIRC photomultipliers, an additional external bucking coil is installed [28]. This is a conventional water cooled copper coil consisting of ten layers. Although the nominal operating current is $200 \mathrm{~A}$, a current of up to $575 \mathrm{~A}$ is attainable, if needed, to demagnetize the DIRC shield.
To optimally control the stray fields and avoid a magnetization of the DIRC magnetic shield, the currents in the solenoid and the bucking coil are ramped together under computer control. High precision transducers are used to measure the currents and provide the feedback signals to the power supplies. The values of the currents are recorded in the BABAR database.

\subsection{Magnetic Field Map}

The goal of the magnetic field mapping and subsequent corrections was to determine the magnetic field in the tracking volume to a precision of $0.2 \mathrm{mT}$.

\subsubsection{Mapping Procedure}

A field mapping device was built specifically for the BABAR magnet based on a design concept developed at Fermilab [29]. The magnetic field sensors were mounted on a propeller at the end of a long cantilevered spindle which reached through the hole in the forward end door. The spindle in turn rode on a carriage which moved on precisionaligned rails. The propeller rotated to sample the magnetic volume in $\phi$, and the carriage moved along its axis to cover $z$. Measurements were obtained from five sets of $B_{r}$ and $B_{z}$ and two $B_{\phi}$ Hall probes, all of which were mounted on a plate at different radial positions. This plate was attached to the propeller and its position could be changed to cover the desired range in the radial distance $r$ from the axis. Precision optical alignment tools were used to determine the position of the sensors transverse to the $z$-axis.

The $B_{r}$ and $B_{z}$ probes were two-element devices with a short-term (few month) precision of $0.01 \%$, the $B_{\phi}$ probes were single element devices with a precision of $0.1 \%$ [30]. In addition to the Hall probes, an NMR probe [31] was mounted at a radius of $89 \mathrm{~mm}$ on the propeller to provide a very precise field reference near the $z$-axis as a function of $z$ for $|z|<1000 \mathrm{~mm}$, where $z=0$ at the magnet center. The NMR measurements set the absolute scale of the magnetic field.

The magnetic field was mapped at the nominal central operating field of $1.5 \mathrm{~T}$, as well as at $1.0 \mathrm{~T}$. Measurements were recorded in $100 \mathrm{~mm}$ intervals from -1800 to $+1800 \mathrm{~mm}$ in $z$, and in 
24 azimuthal positions spaced by $15^{\circ}$ for each of three different radial positions of the Hall probe plate. Thus for each $z$ and $\phi$ position, the components $B_{r}$ and $B_{z}$ were measured at 13 distinct radii from $130 \mathrm{~mm}$ to $1255 \mathrm{~mm}$ and $B_{\phi}$ at six radii between $505 \mathrm{~mm}$ and $1180 \mathrm{~mm}$.

The field map was parameterized in terms of a polynomial of degree up to 40 in $r$ and $z$ plus additional terms to account for expected perturbations [32]. The fit reproduced the measurements to within an average deviation of $0.2 \mathrm{mT}$ throughout the tracking volume. The fitting procedure also served as a means of detecting and removing questionable measurements.

\subsubsection{Perturbations to the Field Map}

During the mapping process, the permanent magnet dipoles (B1) and quadrupoles (Q1) were not yet installed. Their presence inside the solenoid results in field perturbations of two kinds. The first is due to the fringe fields of the B1 and Q1 permanent magnets, and of the dipole and quadrupole trim coils mounted on Q1. The B1 field strength reaches a maximum of $\sim 20 \mathrm{mT}$ close to the surface of the B1 casing and decreases rapidly with increasing radius. The fields associated with the trim coils were measured and parameterized prior to installation; they are essentially dipole in character.

The second field perturbation is due to the permeability of the permanent magnet material. Sintered samarium-cobalt has a relative permeability of 1.11 to 1.13 in the $z$ direction, and as a result the solenoid field is modified significantly. Probes between the B1 and Q1 magnets at a radius of about $190 \mathrm{~mm}$ measure the effect of the permeability. The field perturbation is obtained from a two-dimensional, finite element analysis which reproduces the $r$ and $z$ dependence of $B_{r}$ and $B_{z}$. The induced magnetization increases $B_{z}$ by about $9 \mathrm{mT}$ at the interaction point; the effect decreases slowly with increasing radius.

\subsubsection{Field Quality}

To illustrate the quality of magnetic field, Figure 14 shows the field components $B_{z}$ and $B_{r}$ as a function of $z$ for various radial distances $r$. In the tracking volume the field is very uniform, the

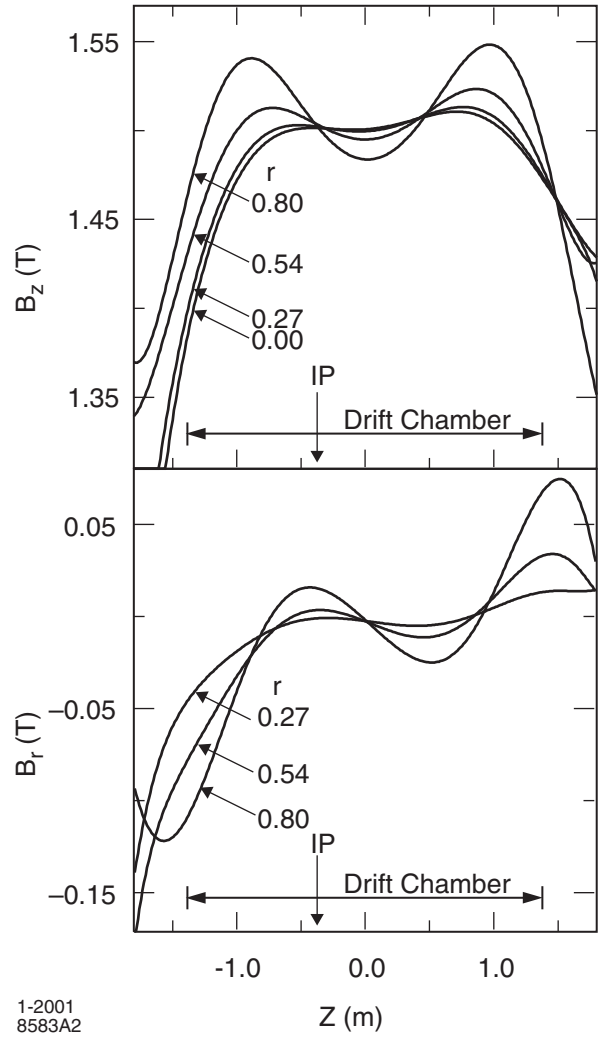

Figure 14. The magnetic field components $B_{z}$ and $B_{r}$ as a function of $z$ for various radial distances $r$ (in $\mathrm{m}$ ). The extent of the $\mathrm{DCH}$ and the location of the interaction point (IP) are indicated.

$B_{\phi}$ component does not exceed $1 \mathrm{mT}$. The variation of the bend field, i.e., the field transverse to the trajectory, along the path of a high momentum track is at most $2.5 \%$ from maximum to minimum within the tracking region, as shown in Figure 15.

\subsubsection{Field Computation}

In order to reduce the computation of the magnetic field for track reconstruction and momentum determination, the field values averaged over azimuth are stored in a grid of $r-z$ space points spanning the volume interior to the cryostat. Local values are obtained by interpolation. Within the volume of the SVT, a linear interpolation is performed in a $20 \mathrm{~mm}$ grid; elsewhere the interpo- 


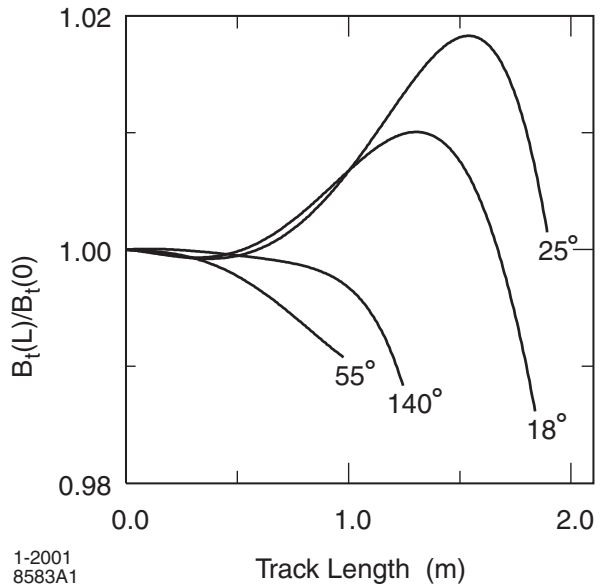

Figure 15. Relative magnitude of magnetic field transverse to a high momentum track as a function of track length from the IP for various polar angles (in degrees). The data are normalized to the field at the origin.

lation is quadratic in a $50 \mathrm{~mm}$ grid. Azimuthal dependence is parameterized by means of a Fourier expansion at each $r-z$ point. The Fourier coefficients at the point of interest are obtained by interpolation on the $r-z$ grid, and the average field value is corrected using the resulting Fourier series.

\subsection{Summary}

Since its successful commissioning, the magnet system has performed without problems. There have been no spontaneous quenches of the superconducting solenoid. In the tracking region, the magnetic field meets specifications, both in magnitude and uniformity. The field compensation and magnetic shielding work well for the DIRC photomultiplier array and the external quadrupoles. Measurements indicate that the bucking coil reduces the field at the face of Q2 from $\sim 50 \mathrm{mT}$ to $\sim 1 \mathrm{mT}$ [28], in agreement with calculations.

\section{Silicon Vertex Tracker}

\subsection{Charged Particle Tracking}

The principal purpose of the BABAR charged particle tracking systems, the SVT and the DCH, is the efficient detection of charged particles and the measurement of their momentum and angles with high precision. Among many applications, these precision measurements allow for the reconstruction of exclusive $B$ - and $D$-meson decays with high resolution and thus minimal background. The reconstruction of multiple decay vertices of weakly decaying $B$ and $D$ mesons is of prime importance to the physics goals.

Track measurements are also important for the extrapolation to the DIRC, EMC, and IFR. At lower momenta, the DCH measurements are more important, while at higher momenta the SVT measurements dominate. Most critical are the angles at the DIRC, because the uncertainties in the charged particle track parameters add to the uncertainty in the measurement of the Cherenkov angle. Thus, the track errors from the combined SVT and DCH measurements should be small compared to the average DIRC Cherenkov angle measurements, i.e., of order of $1 \mathrm{mrad}$, particularly at the highest momenta.

\subsection{SVT Goals and Design Requirements}

The SVT has been designed to provide precise reconstruction of charged particle trajectories and decay vertices near the interaction region. The design choices were driven primarily by direct requirements from physics measurements and constraints imposed by the PEP-II interaction region and $B A B A R$ experiment. In this section the mechanical and electronic design of the SVT are discussed, with some discussion of the point resolution per layer and $d E / d x$ performance. The tracking performance and efficiency of the SVT alone and in combination with the DCH are described in Section 7.

\subsubsection{SVT Requirements and Constraints}

The SVT is critical for the measurement of the time-dependent $C P$ asymmetry. To avoid significant impact of the resolution on the $C P$ asymmetry measurement the mean vertex resolution along the $z$-axis for a fully reconstructed $B$ decay must be better than $80 \mu \mathrm{m}$ [2]. The required resolution in the $x-y$ plane arises from the need to reconstruct final states in $B$ decays as well as in $\tau$ and charm decays. For example, in decays 
of the type $B^{0} \rightarrow D^{+} D^{-}$, separating the two $D$ vertices is important. The distance between the two $D$ 's in the $x-y$ plane for this decay is typically $\sim 275 \mu \mathrm{m}$. Hence, the SVT needs to provide resolution of order $\sim 100 \mu \mathrm{m}$ in the plane perpendicular to the beam line.

Many of the decay products of $B$ mesons have low $p_{\mathrm{t}}$. The SVT must provide standalone tracking for particles with transverse momentum less than $120 \mathrm{MeV} / c$, the minimum that can be measured reliably in the DCH alone. This feature is fundamental for the identification of slow pions from $D^{*}$-meson decays: a tracking efficiency of $70 \%$ or more is desirable for tracks with a transverse momentum in the range $50-120 \mathrm{MeV} / c$. The standalone tracking capability and the need to link SVT tracks to the DCH were crucial in choosing the number of layers.

Beyond the standalone tracking capability, the SVT provides the best measurement of track angles, which is required to achieve design resolution for the Cherenkov angle for high momentum tracks.

Additional constraints are imposed by the storage ring components. The SVT is located inside the $\sim 4.5 \mathrm{~m}$-long support tube, that extends all the way through the detector. To maximize the angular coverage, the SVT must extend down to $350 \mathrm{mrad}\left(20^{\circ}\right)$ in polar angle from the beam line in the forward direction. The region at smaller polar angles is occupied by the B1 permanent magnets. In the backward direction, it is sufficient to extend the SVT sensitive area down to $30^{\circ}$.

The SVT must withstand 2 MRad of ionizing radiation. A radiation monitoring system capable of aborting the beams is required. The expected radiation dose is $1 \mathrm{Rad} /$ day in the horizontal plane immediately outside the beam pipe (where the highest radiation is concentrated), and $0.1 \mathrm{Rad} /$ day on average otherwise.

The SVT is inaccessible during normal detector operations. Hence, reliability and robustness are essential: all components of the SVT inside the support tube should have long mean-time-tofailure, because the time needed for any replacement is estimated to be $4-5$ months. Redundancies are built in whenever possible and practical.

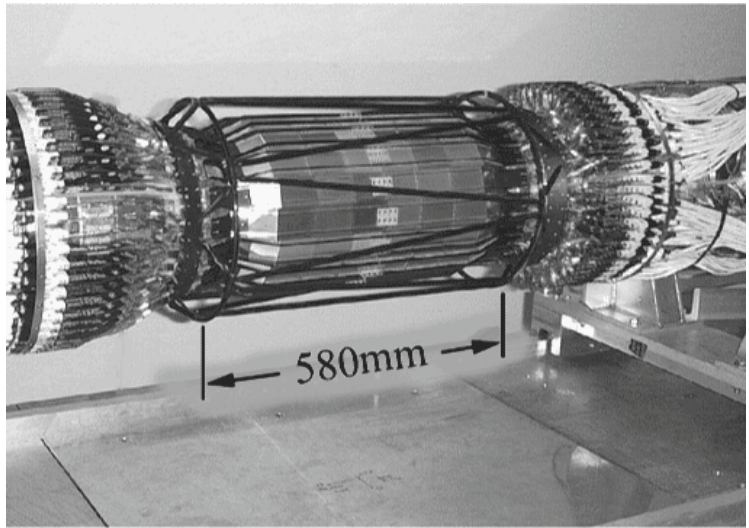

Figure 16. Fully assembled SVT. The silicon sensors of the outer layer are visible, as is the carbonfiber space frame (black structure) that surrounds the silicon.

The SVT is cooled to remove the heat generated by the electronics. In addition, it operates in the $1.5 \mathrm{~T}$ magnetic field.

To achieve the position resolution necessary to carry out physics analyses, the relative position of the individual silicon sensors should be stable over long time periods. The assembly allows for relative motion of the support structures with respect to the $\mathrm{B} 1$ magnets.

These requirements and constraints have led to the choice of a SVT made of five layers of doublesided silicon strip sensors. To fulfill the physics requirements, the spatial resolution, for perpendicular tracks, must be $10-15 \mu \mathrm{m}$ in the three inner layers and about $40 \mu \mathrm{m}$ in the two outer layers. The inner three layers perform the impact parameter measurements, while the outer layers are necessary for pattern recognition and low $p_{\mathrm{t}}$ tracking.

\subsection{SVT Layout}

The five layers of double-sided silicon strip sensors, which form the SVT detector, are organized in $6,6,6,16$, and 18 modules, respectively; a photograph is shown in Figure 16. The strips on the opposite sides of each sensor are oriented orthogonally to each other: the $\phi$ measuring strips $(\phi$ strips) run parallel to the beam and the $z$ measuring strips ( $z$ strips) are oriented transversely to the beam axis. The modules of the inner three 


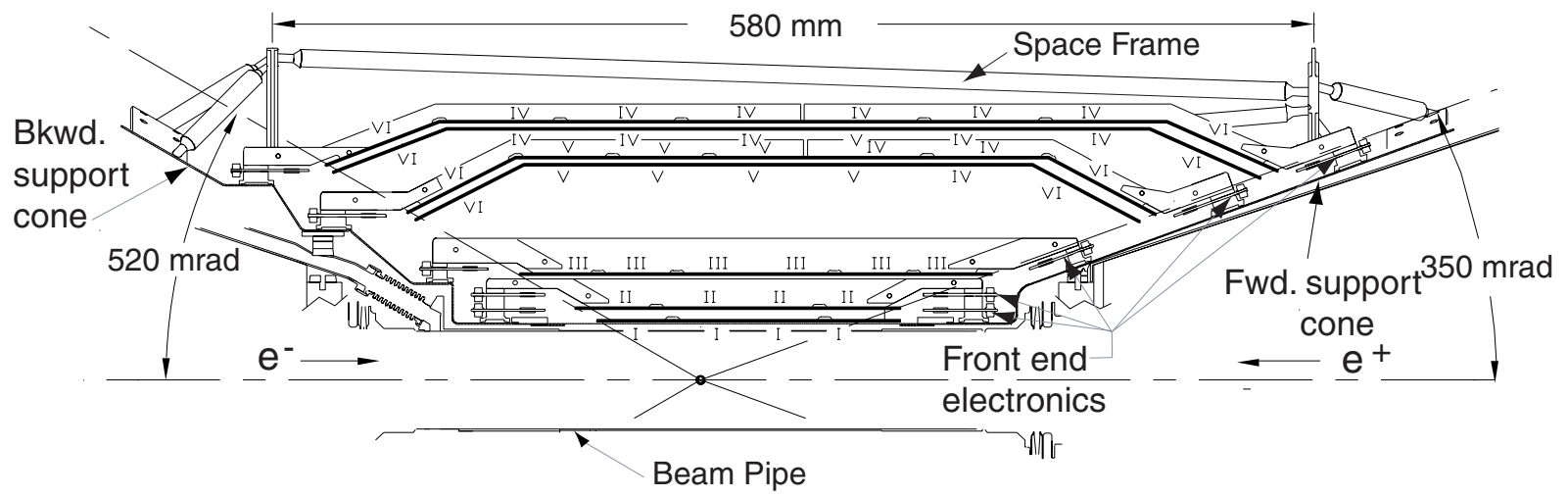

Figure 17. Schematic view of SVT: longitudinal section. The roman numerals label the six different types of sensors.

layers are straight, while the modules of layers 4 and 5 are arch-shaped (Figures 17 and 18).

This arch design was chosen to minimize the amount of silicon required to cover the solid angle, while increasing the crossing angle for particles near the edges of acceptance. A photograph of an outer layer arch module is shown in Figure 19. The modules are divided electrically into two halfmodules, which are read out at the ends.

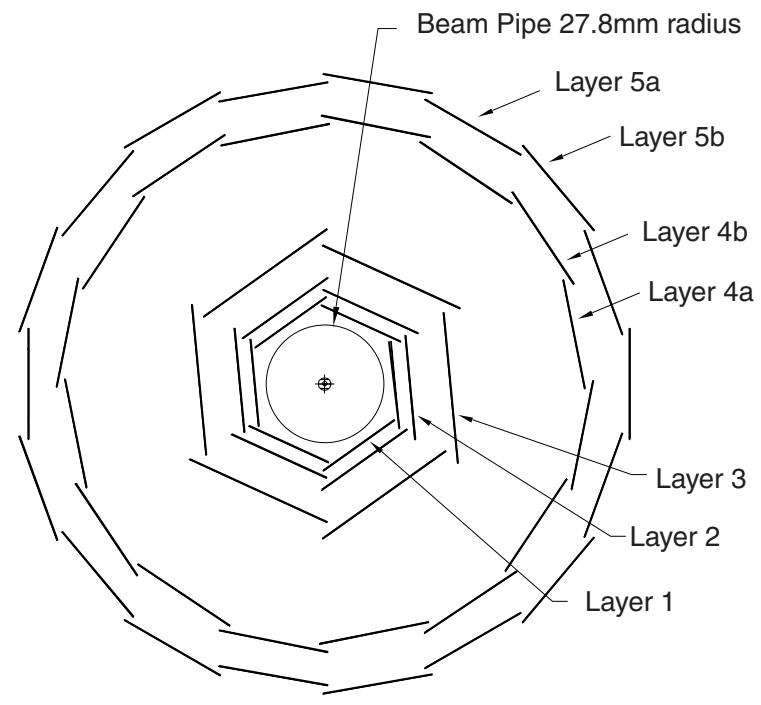

Figure 18. Schematic view of SVT: tranverse section.
To satisfy the different geometrical requirements of the five SVT layers, five different sensor shapes are required to assemble the planar sections of the layers. The smallest detectors are $43 \times 42 \mathrm{~mm}^{2}(z \times \phi)$, and the largest are $68 \times 53 \mathrm{~mm}^{2}$. Two identical trapezoidal sensors are added (one each at the forward and backward ends) to form the arch modules. The halfmodules are given mechanical stiffness by means of two carbon fiber/kevlar ribs, which are visible in Figure 19. The $\phi$ strips of sensors in the same half-module are electrically connected with wire bonds to form a single readout strip. This results in a total strip length up to $140 \mathrm{~mm}(240 \mathrm{~mm})$ in the inner (outer) layers.

The signals from the $z$ strips are brought to the readout electronics using fanout circuits consisting of conducting traces on a thin $(50 \mu \mathrm{m})$ insulating Upilex [33] substrate. For the innermost three layers, each $z$ strip is connected to its own preamplifier channel, while in layers 4 and 5 the number of $z$ strips on a half-module exceeds the number of electronics channels available, requiring that two $z$ strips on different sensors be electrically connected (ganged) to a single electronics channel. The length of a $z$ strip is about $50 \mathrm{~mm}$ (no ganging) or $100 \mathrm{~mm}$ (two strips connected). The ganging introduces an ambiguity on the $z$ coordinate measurement, which must be resolved by the pattern recognition algorithms. The to- 


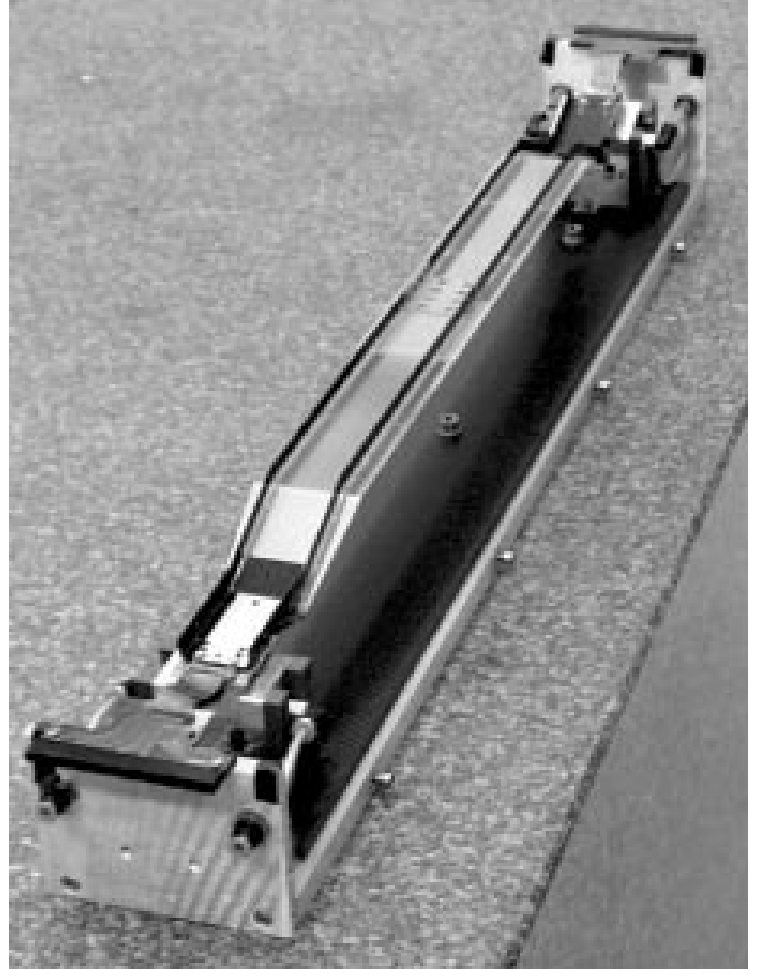

Figure 19. Photograph of an SVT arch module in an assembly jig.

tal number of readout channels is approximately 150,000 .

The inner modules are tilted in $\phi$ by $5^{\circ}$, allowing an overlap region between adjacent modules, a feature that provides full azimuthal coverage and is advantageous for alignment. The outer modules cannot be tilted, because of the arch geometry. To avoid gaps and to have a suitable overlap in the $\phi$ coordinate, layers 4 and 5 are divided into two sub-layers (4a, 4b, 5a, 5b) and placed at slightly different radii (see Figure 18). The relevant geometrical parameters of each layer are summarized in Table 5.

In order to minimize the material in the acceptance region, the readout electronics are mounted entirely outside the active detector volume. The forward electronics must be mounted in the $10 \mathrm{~mm}$ space between the $350 \mathrm{mrad}$ stayclear space and B1 magnet. This implies that the hybrids carrying the front-end chip must be
Table 5

Geometric parameters for each layer and readout plane of the SVT. Floating strips refers to the number of strips between readout (R-O) strips. Note: parts of the $\phi$ sides of layers 1 and 2 are bonded at $100 \mu \mathrm{m}$ and $110 \mu \mathrm{m}$ pitch, respectively, with one floating strip. Strip length of $z$ strips for layers 4 and 5 includes ganging. The radial range for layers 4 and 5 includes the radial extent of the arched sections.

\begin{tabular}{lcccr}
\hline $\begin{array}{l}\text { Layer/ } \\
\text { view }\end{array}$ & $\begin{array}{c}\text { Radius } \\
(\mathrm{mm})\end{array}$ & $\begin{array}{c}\text { R-O } \\
\text { pitch } \\
(\mu \mathrm{m})\end{array}$ & $\begin{array}{c}\text { Floating } \\
\text { strips }\end{array}$ & $\begin{array}{r}\text { Strip } \\
\text { length } \\
(\mathrm{mm})\end{array}$ \\
\hline $1 \mathrm{z}$ & 32 & 100 & 1 & 40 \\
$1 \phi$ & 32 & $50-100$ & $0-1$ & 82 \\
$2 \mathrm{z}$ & 40 & 100 & 1 & 48 \\
$2 \phi$ & 40 & $55-110$ & $0-1$ & 88 \\
$3 \mathrm{z}$ & 54 & 100 & 1 & 70 \\
$3 \phi$ & 54 & 110 & 1 & 128 \\
$4 \mathrm{z}$ & $91-127$ & 210 & 1 & 104 \\
$4 \phi$ & $91-127$ & 100 & 1 & 224 \\
$5 \mathrm{z}$ & $114-144$ & 210 & 1 & 104 \\
$5 \phi$ & $114-144$ & 100 & 1 & 265 \\
\hline
\end{tabular}

positioned at an angle of $350 \mathrm{mrad}$ relative to the sensor for the layers 3,4 , and 5 (Figure 17). In the backward direction, the available space is larger and the inner layer electronics can be placed in the sensor plane, allowing a simplified assembly.

The module assembly and the mechanics are quite complicated, especially for the arch modules, and are described in detail elsewhere [34]. The SVT support structure (Figure 16) is a rigid body made from two carbon-fiber cones, connected by a space frame, also made of carbon-fiber epoxy laminate.

An optical survey of the SVT on its assembly jig indicated that the global error in placement of the sensors with respect to design was $\sim 200 \mu \mathrm{m}$, FWHM. Subsequently, the detector was disassembled and shipped to SLAC, where it was reassembled on the IR magnets. The SVT is attached to the B1 magnets by a set of gimbal rings in such a way as to allow for relative motion of the two B1 magnets while fixing the position of the SVT relative to the forward B1 and the orien- 
tation relative to the axis of both $\mathrm{B} 1$ dipoles. The support tube structure is mounted on the PEPII accelerator supports, independently of $B A B A R$, allowing for movement between the SVT and the rest of BABAR. Precise monitoring of the beam interaction point is necessary, as is described in Section 5.5.

The total active silicon area is $0.96 \mathrm{~m}^{2}$ and the material traversed by particles is $\sim 4 \%$ of a radiation length (see Section 2). The geometrical acceptance of SVT is $90 \%$ of the solid angle in the c.m. system, typically $86 \%$ are used in charged particle tracking.

\subsection{SVT Components}

A block diagram of SVT components is shown in Figure 20. The basic components of the detector are the silicon sensors, the fanout circuits, the Front End Electronics (FEE) and the data transmission system. Each of these components is discussed below.

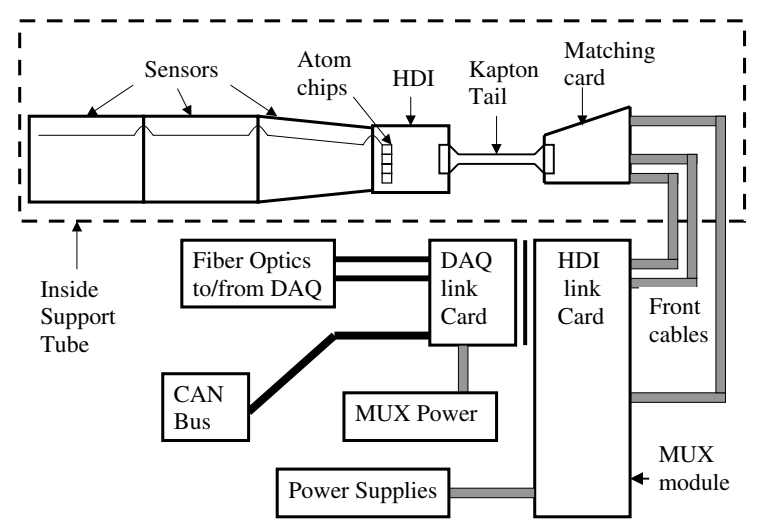

Figure 20. Schematic block diagram showing the different components of the SVT.

\subsubsection{Silicon Sensors}

The SVT sensors [35] are $300 \mu \mathrm{m}$ thick doublesided silicon strip devices. They were designed at INFN Pisa and Trieste (Italy) and fabricated commercially [36]. They are built on highresistivity $(6-15 \mathrm{k} \Omega$-cm) n-type substrates with $\mathrm{p}^{+}$strips and $\mathrm{n}^{+}$strips on the two opposite sides. The insulation of the $\mathrm{n}^{+}$strips is provided by individual p-stops, so as to achieve an inter-strip
Table 6

Electrical parameters of the SVT, shown for the different layers and views. $C_{\text {input }}$ refers to the total input capacitance, $R_{\text {series }}$ is the series resistance. The amplifier peaking time is $200 \mathrm{~ns}$ for layers $1-3$ and $400 \mathrm{~ns}$ for layers $4-5$.

\begin{tabular}{lrrrr}
\hline & & & \multicolumn{2}{c}{ Noise, } \\
$\begin{array}{l}\text { Layer } \\
\text { view }\end{array}$ & $\begin{array}{r}C_{\text {input }} \\
(\mathrm{pF})\end{array}$ & $\begin{array}{r}R_{\text {series }} \\
(\Omega)\end{array}$ & $\begin{array}{r}\text { calc. } \\
(\mathrm{elec})\end{array}$ & $\begin{array}{r}\text { meas. } \\
(\mathrm{elec})\end{array}$ \\
\hline $1 \mathrm{z}$ & 6.0 & 40. & 550 & 880 \\
$1 \phi$ & 17.2 & 164. & 990 & 1200 \\
$2 \mathrm{z}$ & 7.2 & 48. & 600 & 970 \\
$2 \phi$ & 18.4 & 158. & 1030 & 1240 \\
$3 \mathrm{z}$ & 10.5 & 70. & 700 & 1180 \\
$3 \phi$ & 26.8 & 230. & 1470 & 1440 \\
$4 \mathrm{z}$ & 16.6 & 104. & 870 & 1210 \\
$4 \phi$ & 33.6 & 224. & 1380 & 1350 \\
$5 \mathrm{z}$ & 16.6 & 104. & 870 & 1200 \\
$5 \phi$ & 39.7 & 265. & 1580 & 1600 \\
\hline
\end{tabular}

resistance greater than $100 \mathrm{M} \Omega$ at operating bias voltage, normally about $10 \mathrm{~V}$ above the depletion voltage.

Typical depletion voltages are in the range 25$35 \mathrm{~V}$. The strips are biased on both sides with polysilicon resistors $(4-20 \mathrm{M} \Omega$ ) to ensure the required radiation hardness, keeping the voltage drop across resistors and the parallel noise as low as possible. Strips are AC-coupled to the electronics via integrated decoupling capacitors, the capacitance of which depends on the sensor shape, but is always greater than $14 \mathrm{pF} / \mathrm{cm}$. The sensors were designed to maximize the active area, which extends to within $0.7 \mathrm{~mm}$ of the physical edges. Another design goal was to control the inter-strip capacitance: values between $0.7 \mathrm{pF} / \mathrm{cm}$ and $1.1 \mathrm{pF} / \mathrm{cm}$ were obtained for the various sensor shapes. To achieve the required spatial resolution, while keeping the number of readout channels as low as possible, most of the modules have a floating strip (i.e., not read out) between two readout strips.

The leakage currents, because of the excellent performance of the manufacturing process, were as low as $50 \mathrm{nA} / \mathrm{cm}^{2}$ on average, measured at $10 \mathrm{~V}$ above depletion voltage. The silicon sensor 
parameters have been measured after irradiation with ${ }^{60} \mathrm{Co}$ sources. Apart from an increase in the inter-strip capacitance of about $12 \%$ during the first $100 \mathrm{krad}$, the main effect was an increase of the leakage current by $0.7 \mu \mathrm{A} / \mathrm{cm}^{2} / \mathrm{MRad}$. However, in a radiation test performed in a $1 \mathrm{GeV} / c$ electron beam, an increase in leakage current of about $2 \mu \mathrm{A} / \mathrm{cm}^{2} / \mathrm{MRad}$ and a significant shift in the depletion voltage, dependent on the initial dopant concentration, were observed. A shift of about $8-10 \mathrm{~V}$ was seen for irradiation corresponding to a dose of approximately 1 MRad. These observations indicate significant bulk damage caused by energetic electrons. As indicated by the change in depletion voltage, the SVT sensors could undergo type inversion after about 13 MRad. Preliminary tests show that the sensors continue to function after type inversion [37]. Studies of the behavior of SVT modules as a function of radiation dose continue.

\subsubsection{Fanout Circuits}

The fanout circuits, which route the signals from the strips to the electronics, have been designed to minimize the series resistance and the inter-strip capacitance. As described in ref. [38], a trace on the fanout has a series resistance about $1.6 \Omega / \mathrm{cm}$, an inter-strip resistance $>20 \mathrm{M} \Omega$, and an inter-strip capacitance $<0.5 \mathrm{pF} / \mathrm{cm}$. The electrical parameters of the final assembly of sensors and fanouts (referred to as Detector Fanout Assemblies or DFAs) are summarized in Table 6. Due to the different strip lengths, there are large differences between the inner and the outer layers. Smaller differences are also present between the forward and backward halves of the module, that are of different lengths.

\subsubsection{Front End Electronics}

The electrical parameters of a DFA and the general $B A B A R$ requirements are the basic inputs that drove the design of the SVT front-end custom IC; the ATOM (A Time-Over-Threshold Machine). In particular, the front-end IC had to satisfy the following requirements:

- signal to noise ratio greater than 15 for minimum ionizing particle (MIP) signals for all modules;
- signals from all strips must be retained, in order to improve the spatial resolution through interpolation, while keeping the number of transmitted hits as low as possible. A hit refers to a deposited charge greater than $0.95 \mathrm{fC}$, corresponding to 0.25 MIP;

- the amplifier must be sensitive to both negative and positive charge;

- the peaking time must be programmable, with a minimum of $100 \mathrm{~ns}$ (in layers 1 and 2 , because of the high occupancy), up to $400 \mathrm{~ns}$ (outer layers, with high capacitance);

- capability to accept random triggers with a latency up to $11.5 \mu \mathrm{s}$ and a programmable jitter up to $\pm 1 \mu \mathrm{s}$, without dead time;

- radiation hardness greater than 2.5 MRad;

- small dimensions: 128 channels in a $6.2 \mathrm{~mm}$ wide chip.

These requirements are fully satisfied by the ATOM IC [39], which is depicted schematically in Figure 21.

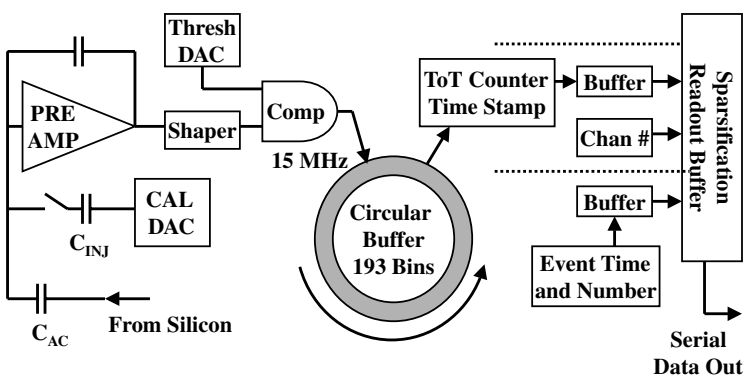

Figure 21. Schematic diagram of the ATOM front end IC.

The linear analog section consists of a chargesensitive preamplifier followed by a shaper. Gains of $200 \mathrm{mV} / \mathrm{fC}$ (low) or $300 \mathrm{mV} / \mathrm{fC}$ (high) may be selected. The channel gains on a IC are uniform to $5 \mathrm{mV} / \mathrm{fC}$. Signals are presented to a programmable-threshold comparator, designed so that the output width of the pulse (Time over 
Table 7

ATOM chip ENC parameters at different peaking times

\begin{tabular}{ccc}
\hline $\begin{array}{c}\text { Peaking } \\
\text { time }\end{array}$ & $\begin{array}{c}\text { ENC } \\
(0 \mathrm{pF})\end{array}$ & $\begin{array}{c}\text { Noise } \\
\text { slope }\end{array}$ \\
\hline $100 \mathrm{~ns}$ & $380 e^{-}$ & $40.9 e^{-} / \mathrm{pF}$ \\
$200 \mathrm{~ns}$ & $280 e^{-}$ & $33.9 e^{-} / \mathrm{pF}$ \\
$400 \mathrm{~ns}$ & $220 e^{-}$ & $25.4 e^{-} / \mathrm{pF}$ \\
\hline
\end{tabular}

Threshold or ToT) is a quasi-logarithmic function of the collected charge. This output is sampled at $30 \mathrm{MHz}$ and stored in a 193 location buffer. Upon receipt of a Level 1 (L1) trigger, the time and ToT is retrieved from this latency buffer, sparcified, and stored in a four event buffer. Upon the receipt of an $L 1$ Accept command from the data acquisition system, the output data (the 4 bits for the ToT, 5 bits for the time stamp, and 7 bits for the strip address) are formatted, serialized, and delivered to the ROM. The IC also contains a test charge injection circuit. The typical noise behavior of the ATOM, as described by the Equivalent Noise Charge (ENC) of the linear analog section is given in Table 7 .

The average noise for the various module types is shown in Table 6. Given that shot noise due to sensor leakage current is negligible, the expected noise may be calculated from the parameters of Tables 6 and 7 . The results of such a calculation are also shown in Table 6 . The maximum average noise is 1,600 electrons, leading to a signal-tonoise ratio greater than 15 .

The power consumption of the IC is about $4.5 \mathrm{~mW} /$ channel. Radiation hardness was studied up to $2.4 \mathrm{MRad}$ with a ${ }^{60} \mathrm{Co}$ source. At that dose, the gain decreased $20 \%$, and the noise increased less than $15 \%$.

The ATOM ICs are mounted on thick-film double-sided hybrid circuits (known as High Density Interconnects or HDIs) based on an aluminum-nitride substrate with high thermal conductivity. The electronics are powered through a floating power supply system, in such a way as to guarantee a small voltage drop $(<1 \mathrm{~V})$ across the detector decoupling capacitors.

\subsubsection{Data Transmission}

The digitized signals are transmitted from the ATOM ICs through a thin kapton tail or cable to the matching cards, from where they are routed to more conventional cables. Just outside the detector, signals are multiplexed by the MUX modules, converted into optical signals and transmitted to the Readout Modules (ROMs). The MUX modules also receive digital signals from the DAQ via a fiber optical connection. The SVT is connected to the BABAR online detector control and monitoring system via the industry standard CAN bus. Details on SVT data transmission system and DAQ can be found in references [40,41]. Power to SVT modules (silicon sensor bias voltage and ATOM low voltages) is provided by a CAEN A522 power supply system [42].

\subsection{Monitoring and Calibration}

To identify immediately any operational problems, the SVT is integrated in the control and monitoring system (see Section 12). Major concerns for SVT monitoring are temperature and humidity, mechanical position, and radiation dose.

\subsubsection{Temperature and Humidity Monitors}

The total power dissipation of the SVT modules is about $350 \mathrm{~W}$, mainly dissipated in the ATOM ICs. External cooling is provided by chilled water at $8^{\circ} \mathrm{C}$. In addition, humidity is reduced by a stream of dry air in the support tube.

Since condensation or excessive temperature can permanently damage the FEE, temperature and humidity monitoring are very important to the safe operation of the SVT. Thermistors are located on the HDIs (for the measurements of FEE temperature), around the SVT, along the cooling systems, and in the electronics (MUX) crates. The absolute temperatures are monitored to $0.2^{\circ} \mathrm{C}$ and relative changes of $0.1^{\circ} \mathrm{C}$. Additionally, a series of humidity sensors are employed to monitor the performance of the dry air system. The temperature and humidity monitors also serve as an interlock to the HDI power supplies. 


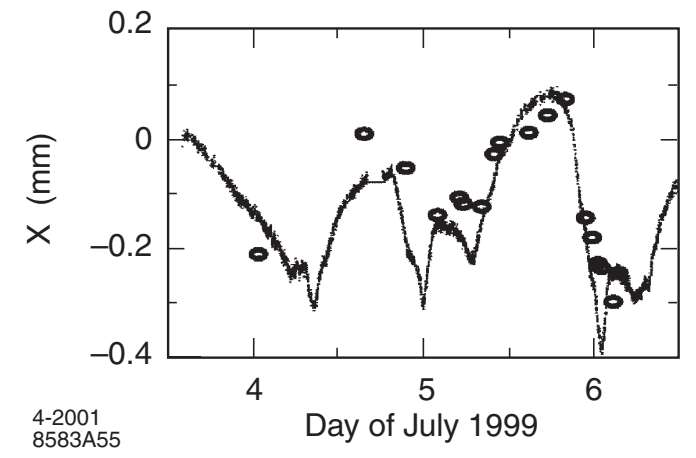

Figure 22. Horizontal motion between the DCH and the support tube measured with the capacitive sensors (curve) compared to the mean $x$ coordinate of the interaction point (circles) measured with $e^{+} e^{-}$and $\mu^{+} \mu^{-}$events over a three-day period in July 1999. An arbitrary offset and scale has been applied to the beam position data.

\subsubsection{Position Monitors}

A system of capacitive sensors was installed to identify and track changes in the position of the SVT with respect to the PEP-II B1 magnets and the position of the support tube with respect to the DCH. An example of the understanding that can be achieved by this system is given in Figure 22, where the measured changes in the horizontal position of the SVT relative to the $\mathrm{DCH}$ are shown for a period of six day in the summer of 1999. These position changes can be attributed to local temperature variations. The sensor data are compared to measurements of the mean position of the interaction point (in the horizontal plane) determined with $e^{+} e^{-}$and $\mu^{+} \mu^{-}$events recorded over this period. While the amplitude of motion at the time was uncharacteristically large, the strong correlation between these independent measurements is quite evident. Alignment with charged particle tracks is now performed routinely to correct for relative motion of the tracking systems, as described in Section 5.6 .2 .

\subsubsection{Radiation Monitors}

Radiation monitoring is extremely important to ensure the SVT does not exceed its radiation budget, which could cause permanent damage to the device. To date, the measured radiation absorbed by the SVT is well within the allowed budget.

The monitoring of radiation dose to the SVT is discussed in detail in Section 3.

\subsubsection{Calibrations}

Once a day, and each time the SVT configuration has changed, calibrations are performed in absence of circulating beams. All electronic channels are tested with pulses through test capacitors, for different values of the injected charge. Gains, thresholds, and electronic noise are measured, and defective channels are identified. The calibration results have proven very stable and repeatable. The main variation in time is the occasional discovery of a new defective channel. The calibration procedures have also been very useful for monitoring noise sources external to the SVT.

\subsubsection{Defects}

Due to a series of minor mishaps incurred during the installation of the SVT, nine out of 208 readout sections (each corresponding to one side of a half-module) were damaged and are currently not functioning. There is no single failure mode, but several causes: defective connectors, mishandling during installation, and notfully-understood problems on the FEE hybrid. There has been no module failure due to radiation damage. It should be noted that due to the redundancy afforded by the five layers of the SVT, the presence of the defective modules has minimal impact on physics analyses.

In addition, there are individual channel defects, of various types, at a level of about 1\%. Calibrations have revealed an increase in the number of defective channels at a rate of less than $0.2 \% /$ year.

\subsection{Data Analysis and Performance}

This section describes the reconstruction of space points from signals in adjacent strips on both sides of the sensors, the SVT internal and global alignment, single hit efficiency, and resolution and $d E / d x$ performance of the SVT. 


\subsubsection{Cluster and Hit Reconstruction}

Under normal running conditions, the average occupancy of the SVT in a time window of $1 \mu \mathrm{s}$ is about $3 \%$ for the inner layers, with a significant azimuthal variation due to beam-induced backgrounds, and less than $1 \%$ for the outer layers, where noise hits dominate. Figure 23 shows the typical occupancy as a function of IC index (equivalent to azimuthal angle, in this case) for layer $1, \phi$ side. In the inner layers, the occupancy is dominated by machine backgrounds, which are significantly higher in the horizontal plane, seen in the plot as the peaks near IC indices 3 and 25 .

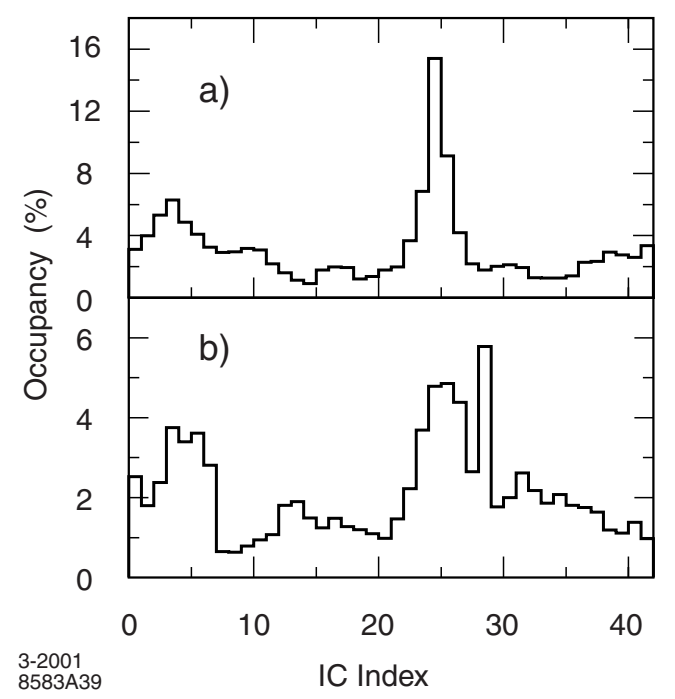

Figure 23. Typical occupancy in percent as a function of IC index in layer $1, \phi$ side for a) forward half-modules and b) backward halfmodules. The IC index increases with azimuthal angle and the higher occupancy in the horizontal plane is visible near chip indices 3 and 25 .

The first step of the reconstruction program consists in discarding out-of-time channels. A time correction, i.e., the time between the passage of the particle and the time the shaper exceeds threshold, is performed, after which hits with times more than $200 \mathrm{~ns}$ from the event time (determined by the DCH) are discarded. The loss of real hits from this procedure is negligible. The resulting in-time hits are then passed to the cluster finding algorithm. First, the charge pulse height (Q) of a single pulse is calculated from the ToT value, and clusters are formed grouping adjacent strips with consistent times. In a second pass, clusters separated by just one strip are merged into one cluster. The two original clusters plus the merged cluster are made available to the pattern recognition algorithm, which chooses among the three.

The position $x$ of a cluster formed by $n$ strips is determined, with the "head-to-tail" algorithm:

$x=\frac{\left(x_{1}+x_{n}\right)}{2}+\frac{p}{2} \frac{\left(Q_{n}-Q_{1}\right)}{\left(Q_{n}+Q_{1}\right)}$,

where $x_{i}$ and $Q_{i}$ are the position and collected charge of $i$-th strip, respectively, and $p$ is the readout pitch. This formula results in a cluster position that is always within $p / 2$ of the geometrical center of the cluster. The cluster pulse height is simply the sum of the strip charges, while the cluster time is the average of the signal times.

\subsubsection{Alignment}

The alignment of the SVT is performed in two steps. The first step consists of determining the relative positions of the 340 silicon sensors. Once this is accomplished, the next step is to align the SVT as a whole within the global coordinate system defined by the $\mathrm{DCH}$. The primary reason for breaking the alignment procedure into these two steps is that the local positions are relatively stable in time compared to the global position. Also, the local alignment procedure is considerably more complex than the global alignment procedure. Thus, the global alignment can be updated on a run-by-run basis, while the local alignment constants are changed as needed, typically after magnet quenches or detector access.

The local alignment procedure is performed with tracks from $e^{+} e^{-} \rightarrow \mu^{+} \mu^{-}$events and cosmic rays. Well isolated, high momentum tracks from hadronic events are also used to supplement di-muon and cosmic data. Data samples sufficient to perform the local alignment are collected in one to two days of typical running conditions.

In $\mu^{+} \mu^{-}$events, the two tracks are simultaneously fit using a Kalman filter technique and the known beam momentum. The use of tracks 
from cosmic rays reduces any systematic distortion that may be introduced due to imprecise knowledge of the beam momenta. No information from the DCH is used, effectively decoupling the SVT and DCH alignment.

In addition to the information from tracks, data from an optical survey performed during the assembly of the SVT are included in the alignment procedure. The typical precision of these optical measurements is $4 \mu \mathrm{m}$. This survey information is only used to constrain sensors relative to other sensors in the same module, but not one module to another or one layer to another. Furthermore, only degrees of freedom in the plane of the sensor are constrained as they are expected to be the most stable, given the assembly procedure.

Using the hit residuals from the aforementioned set of tracks and the optical survey information, a $\chi^{2}$ is formed for each sensor and minimized with respect to the sensor's six local parameters. The constraints coming from the overlapping regions of the silicon sensors, the di-muon fit, the cosmic rays, and the optical survey result in internally consistent local alignment constants.

Figure 24 shows a comparison between the optical alignment made during the SVT assembly in February 1999 and a local alignment using data taken during January 2000. The alignment from tracking data was made without using cosmics or constraints from the optical survey. The width of the distributions in these plots has four contributions: 1) displacement during the transfer of the SVT from the assembly jig to the IR magnets, 2) time dependent motion of the SVT after mounting, 3) statistical errors, and 4) systematic errors. The second set of plots shows the difference in two alignment sets for data taken in January 2000 as compared to March 2000. In general, the stability of the inner three layers is excellent, with slightly larger tails in the outer two layers. The radial coordinate is less tightly constrained in all measurements because the radial location of the charge deposition is not well known, and most of the information about the radial locations comes only from constraints in the overlap region of the sensors.

After the internal alignment, the SVT is considered as a rigid body. The second alignment

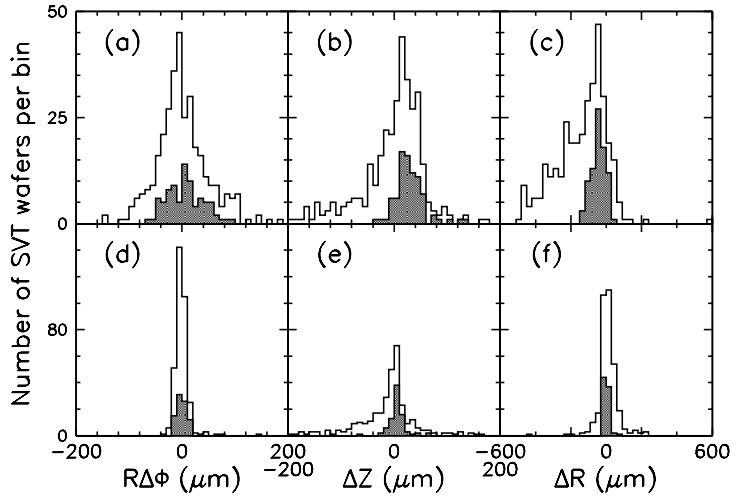

Figure 24. Comparison of a local alignment of all the sensors in the SVT using data from January 2000 with the optical survey of the SVT made during assembly in February 1999 in the (a) $r \Delta \phi$, (b) $\Delta z$ and (c) $\Delta r$ coordinates. Plots (d), (e), and (f) show the difference between two local alignments using data from January 15-19 and March 6-7, 2000 for the $r \Delta \phi, \Delta z$, and $\Delta r$ coordinates, respectively. In all the plots, the shaded regions correspond to the sensors in the first three layers. In comparing the different alignments and optical survey, a six parameter fit (three global translations and three global rotations) has been applied between the data sets.

step consists in determining the position of the SVT with respect to the DCH. Tracks with sufficient numbers of SVT and DCH hits are fit two times: once using only the DCH information and again using only the SVT hits. The six global alignment parameters, three translations and three rotations, are determined by minimizing the difference between track parameters obtained with the SVT-only and the DCH-only fits. As reported above, because of the diurnal movement of the SVT with respect to the DCH, this global alignment needs to be performed once per run ( $\sim$ every $2-3$ hours). The alignment constants obtained in a given run are then used to reconstruct the data in the subsequent run. This procedure, known as rolling calibration, ensures that track reconstruction is always performed with up-to-date global alignment constants.

A record of the changes in the relative posi- 

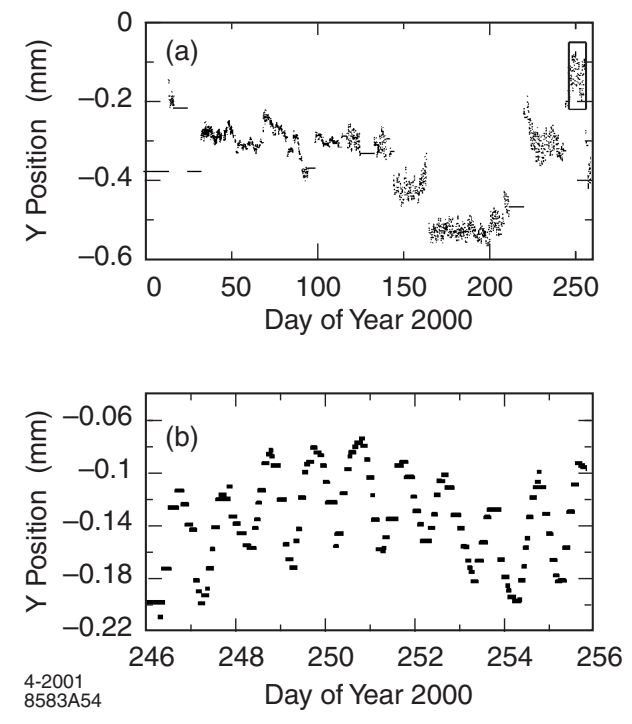

Figure 25. Global alignment of the SVT relative to the $\mathrm{DCH}$ based on $e^{+} e^{-}$and $\mu^{+} \mu^{-}$events: changes in the relative vertical placement measured a) over the entire ten-month run in the year 2000 , and b) a ten-day period, illustrating diurnal variations.

tion of the SVT as determined by rolling calibrations is shown in Figure 25. The position is stable to better than $\pm 100 \mu \mathrm{m}$ over several weeks, but changes abruptly from time to time, for instance, during access to the detector. The calibrations track diurnal variations of typically $\pm 50 \mu \mathrm{m}$ that have been correlated with local changes in temperature of about $\pm 2^{\circ} \mathrm{C}$. Movements within a single run are small compared to the size of the beam.

\subsubsection{Performance}

The SVT efficiency can be calculated for each half-module by comparing the number of associated hits to the number of tracks crossing the active area of the module. As can be seen in Figure 26, a combined hardware and software efficiencies of $97 \%$ is measured, excluding defective readout sections (9 out of 208), but employing no special treatment for other defects, such as broken AC coupling capacitors or dead channels on front-end chips. Actually, since most of the

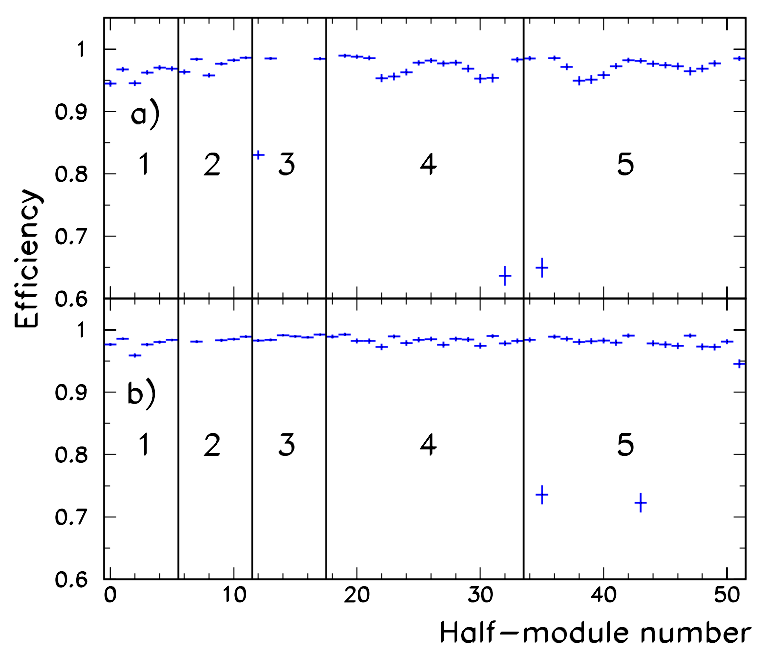

Figure 26. SVT hit reconstruction efficiency, as measured on $\mu^{+} \mu^{-}$events for a) forward halfmodules and b) backward half-modules. The plots show the probability of associating both a $\phi$ and $z$ hit to a track passing through the active part of the detector. The horizontal axis corresponds to the different modules, with the vertical lines separating the different layers as numbered. Missing values correspond to nonfunctioning half-modules.

defects affect a single channel, they do not contribute to the inefficiency, because most tracks deposit charge in two or more strips due to track crossing angles, and charge diffusion.

The spatial resolution of SVT hits is determined by measuring the distance (in the plane of the sensor) between the track trajectory and the hit, using high-momentum tracks in two prong events. The uncertainty due to the track trajectory is subtracted from the width of the residual distribution to obtain the hit resolution. Figure 27 shows the SVT hit resolution for $z$ and $\phi$ side hits as a function of track incident angle, for each of the five layers. The measured resolutions are in excellent agreement with expectations from Monte Carlo simulations.

Initial studies have shown that hit reconstruction efficiency and spatial resolution are effec- 

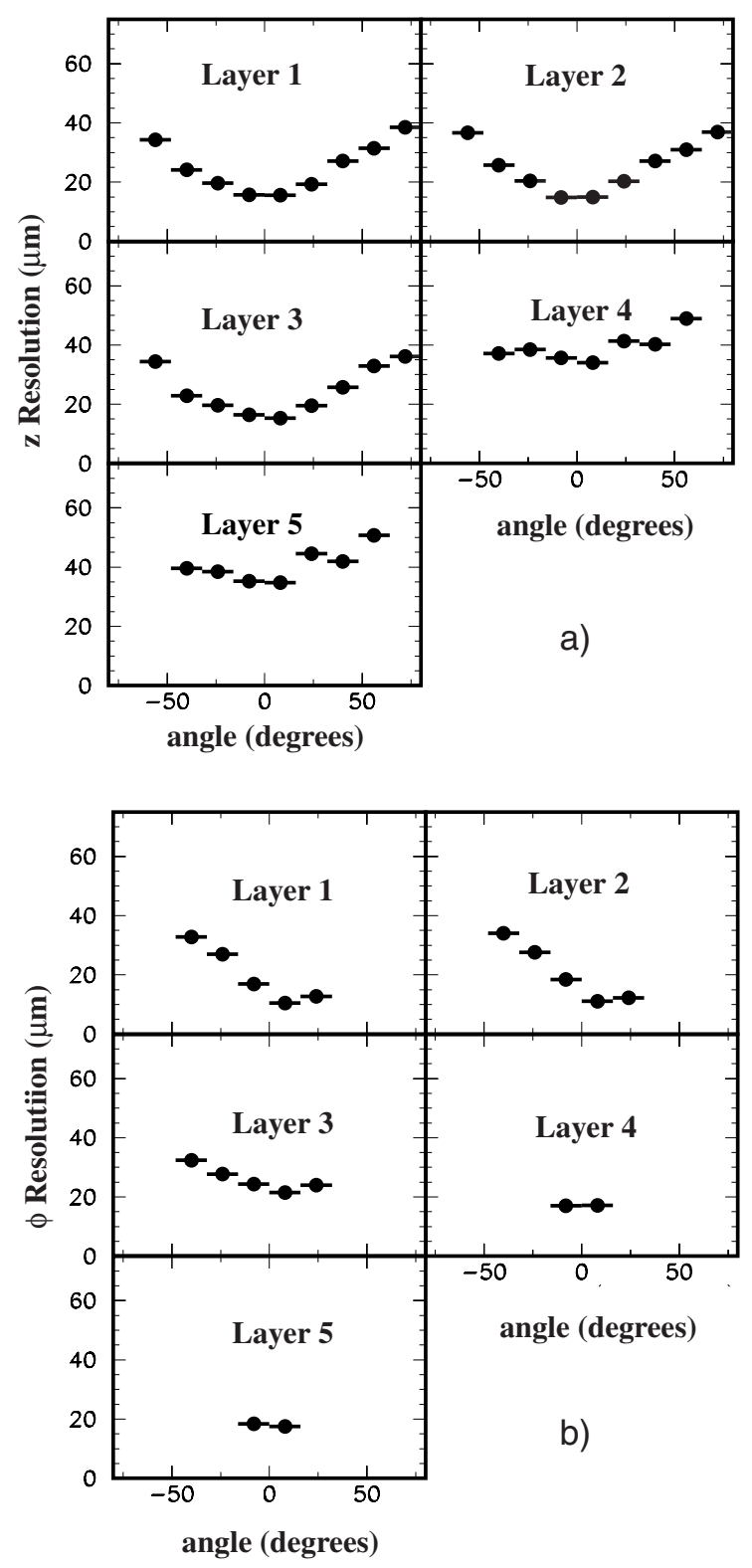

Figure 27. SVT hit resolution in the a) $z$ and b) $\phi$ coordinate in microns, plotted as a function of track incident angle in degrees. Each plot shows a different layer of the SVT. The plots in the $\phi$ coordinate for layers 1-3 are asymmetric around $\phi=0$ because of the "pinwheel" design of the inner layers. There are fewer points in the $\phi$ resolution plots for the outer layers as they subtend smaller angles than the inner layers. tively independent of occupancy for the occupancy levels observed so far.

Measurement of the ToT value by the ATOM ICs enables one to obtain the pulse height, and hence the ionization $d E / d x$ in the SVT sensor. The values of ToT are converted to pulse height using a lookup table computed from the pulse shapes obtained in the bench measurements. The pulse height is corrected for track length variation. The double-sided sensors provide up to ten measurements of $d E / d x$ per track. For every track with signals from at least four sensors in the SVT, a $60 \%$ truncated mean $d E / d x$ is calculated. The cluster with the smallest $d E / d x$ energy is also removed to reduce sensitivity to electronics noise. For MIPs, the resolution on the truncated mean $d E / d x$ is approximately $14 \%$. A $2 \sigma$ separation between the kaons and pions can be achieved up to momentum of $500 \mathrm{MeV} / c$, and between kaons and protons beyond $1 \mathrm{GeV} / c$.

\subsection{Summary and Outlook}

The SVT has been operating efficiently since its installation in the BABAR experiment in May 1999. The five layer device, based on doublesided silicon sensors, has satisfied the original design goals, in particular the targets specified for efficiency, hit resolution, and low transverse momentum track reconstruction. The radiation dose during the first $25 \mathrm{fb}^{-1}$ of integrated luminosity is within the planned budget, and no modules have failed due to radiation damage. The performance of the SVT modules at high radiation dose is currently being studied. Early results indicate that the sensors will continue to function after type inversion (at 1-3 MRad), but further tests with irradiated sensors and ATOM ICs need to be performed. A program of spare module production has commenced, with the goal of replacing modules that are expected to fail due to radiation damage. Beam-generated backgrounds are expected to rise with increasing luminosity. Physics studies at five times the current backgrounds levels indicate no change in mass or vertex resolution for the mode $B^{0} \rightarrow J / \psi K_{S}^{0}$ and a $\sim 20 \%$ loss of resolution in the $D^{*+}-D^{0}$ mass difference. In this study the detector efficiency for both decay modes was lower by $15-20 \%$. 


\section{Drift Chamber}

\subsection{Purpose and Design Requirements}

The principal purpose of the drift chamber $(\mathrm{DCH})$ is the efficient detection of charged particles and the measurement of their momenta and angles with high precision. These high precision measurements enable the reconstruction of exclusive $B$ - and $D$-meson decays with minimal background. The DCH complements the measurements of the impact parameter and the directions of charged tracks provided by the SVT near the IP. At lower momenta, the DCH measurements dominate the errors on the extrapolation of charged tracks to the DIRC, EMC, and IFR.

The reconstruction of decay and interaction vertices outside of the SVT volume, for instance the $K_{S}^{0}$ decays, relies solely on the DCH. For this purpose, the chamber should be able to measure not only the transverse momenta and positions, but also the longitudinal position of tracks, with a resolution of $\sim 1 \mathrm{~mm}$.

The DCH also needs to supply information for the charged particle trigger with a maximum time jitter of $0.5 \mu \mathrm{s}$ (Section 11).

For low momentum particles, the DCH is required to provide particle identification by measurement of ionization loss $(d E / d x)$. A resolution of about $7 \%$ will allow $\pi / K$ separation up to $700 \mathrm{MeV} / c$. This capability is complementary to that of the DIRC in the barrel region, while in the extreme backward and forward directions, the $\mathrm{DCH}$ is the only device providing some discrimination of particles of different mass.

Since the average momentum of charged particles produced in $B$ - and $D$-meson decays is less than $1 \mathrm{GeV} / c$, multiple scattering is a significant, if not the dominant limitation on the track parameter resolution. In order to reduce this contribution, material in front of and inside the chamber volume has to be minimized.

Finally, the DCH must be operational in the presence of large beam-generated backgrounds, which were predicted to generate rates of $\sim 5 \mathrm{kHz} /$ cell in the innermost layers.

\subsection{Mechanical Design and Assembly 6.2.1. Overview}

The DCH is relatively small in diameter, but almost $3 \mathrm{~m}$ long, with 40 layers of small hexagonal cells providing up to 40 spatial and ionization loss measurements for charged particles with transverse momentum greater than $180 \mathrm{MeV} / c$. Longitudinal position information is obtained by placing the wires in 24 of the 40 layers at small angles with respect to the $z$-axis. By choosing low-mass aluminum field wires and a helium-based gas mixture, the multiple scattering inside the $\mathrm{DCH}$ is held to a minimum, less than $0.2 \% X_{0}$ of material. The properties of the chosen gas, a 80:20 mixture of helium:isobutane, are presented in Table 8 . This mixture has a radiation length that is five times larger than commonly used argonbased gases. The smaller Lorentz angle results in a rather uniform time-distance relationship and thereby improved spatial resolution.

Table 8

Properties of helium-isobutane gas mixture at atmospheric pressure and $20^{\circ} \mathrm{C}$. The drift velocity is given for operation without magnetic field, while the Lorentz angle is stated for a $1.5 \mathrm{~T}$ magnetic field.

\begin{tabular}{ll}
\hline Parameter & Values \\
\hline Mixture He $: \mathrm{C}_{4} \mathrm{H}_{10}$ & $80: 20$ \\
Radiation Length & $807 \mathrm{~m}$ \\
Primary Ions & $21.2 / \mathrm{cm}$ \\
Drift Velocity & $22 \mu \mathrm{m} / \mathrm{ns}$ \\
Lorentz Angle & $32^{\circ}$ \\
$d E / d x$ Resolution & $6.9 \%$ \\
\hline
\end{tabular}

The inner cylindrical wall of the $\mathrm{DCH}$ is kept thin to facilitate the matching of the SVT and DCH tracks, to improve the track resolution for high momentum tracks, and to minimize the background from photon conversions and interactions. Material in the outer wall and in the forward direction is also minimized so as not to degrade the performance of the DIRC and the EMC. For this reason, the HV distribution and all of the readout electronics are mounted on the 


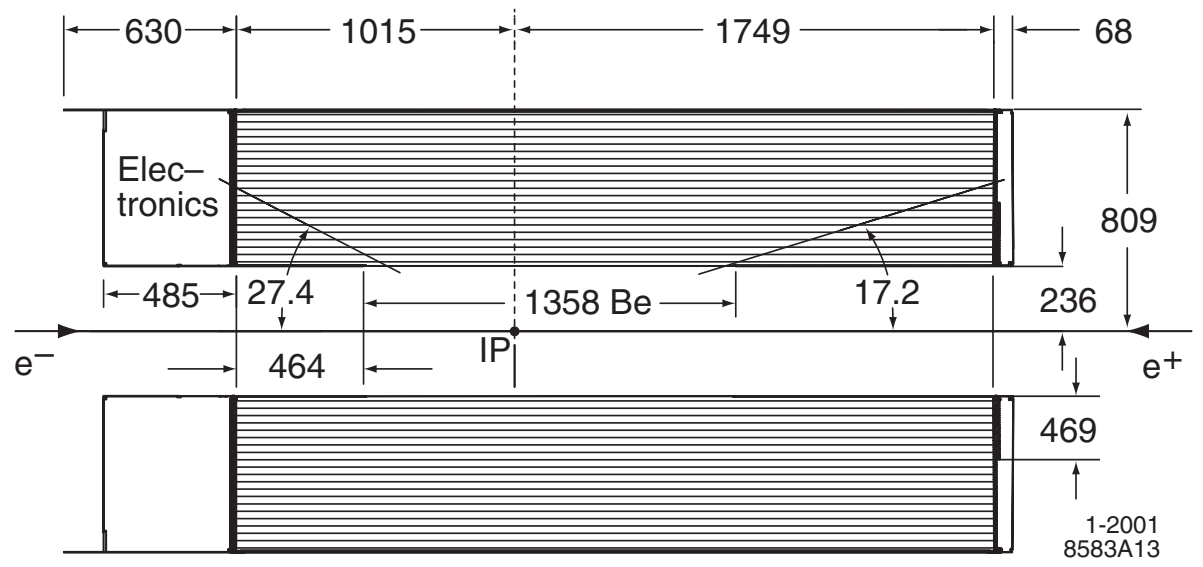

Figure 28. Longitudinal section of the DCH with principal dimensions; the chamber center is offset by $370 \mathrm{~mm}$ from the interaction point (IP).

backward endplate of the chamber. This choice also eliminates the need for a massive, heavily shielded cable plant.

A longitudinal cross section and dimensions of the DCH are shown in Figure 28. The DCH is bounded radially by the support tube at its inner radius and the DIRC at its outer radius. The device is asymmetrically located with respect to the IP. The forward length of $1749 \mathrm{~mm}$ is chosen so that particles emitted at polar angles of $17.2^{\circ}$ traverse at least half of the layers of the chamber before exiting through the front endplate. In the backward direction, the length of $1015 \mathrm{~mm}$ means that particles with polar angles down to $152.6^{\circ}$ traverse at least half of the layers. This choice ensures sufficient coverage for forward-going tracks, and thus avoids significant degradation of the invariant mass resolution, while at the same time maintaining a good safety margin on the electrical stability of the chamber. The DCH extends beyond the endplate by $485 \mathrm{~mm}$ at the backward end to accommodate the readout electronics, cables, and an rf shield. It extends beyond the forward endplate by $68 \mathrm{~mm}$ to provide space for wire feed-throughs and an rf shield.

\subsubsection{Structural Components}

Details of the DCH mechanical design are presented in Figure 29. The endplates, which carry an axial load of $31,800 \mathrm{kN}$, are made from alu- minum plates of $24 \mathrm{~mm}$ thickness. At the forward end, this thickness is reduced to $12 \mathrm{~mm}$ beyond a radius of $46.9 \mathrm{~cm}$ to minimize the material in front of the calorimeter endcap. For this thickness, the estimated safety margin on the plastic yield point for endplate material (6061T651 aluminum) is not more than a factor of two. The maximum total deflection of the endplates under loading is small, about $2 \mathrm{~mm}$ or $28 \%$ of the $7 \mathrm{~mm}$ wire elongation under tension. During installation of the wires, this small deflection was taken into account by over-tensioning the wires.

The inner and outer cylinder cylindrical walls are load bearing to reduce the maximum stress and deflections of the endplates. The stepped forward endplate created a complication during the assembly, because the thinner forward endplate would deflect more than the thicker backward endplate. The outside rim of the forward endplate had to be pre-loaded, i.e., displaced by $2.17 \mathrm{~mm}$ in the forward direction, to maintain the inside and outside rims of the rear endplate at the same longitudinal position after the load of the wires was transfered from the stringing fixture to the outer cylinder.

Prior to installation on the inner cylinder, the two endplates were inspected on a coordinatemeasuring machine. All sense wire holes, as well as $5 \%$ of the field and clearing field wire holes, were measured to determine their absolute loca- 


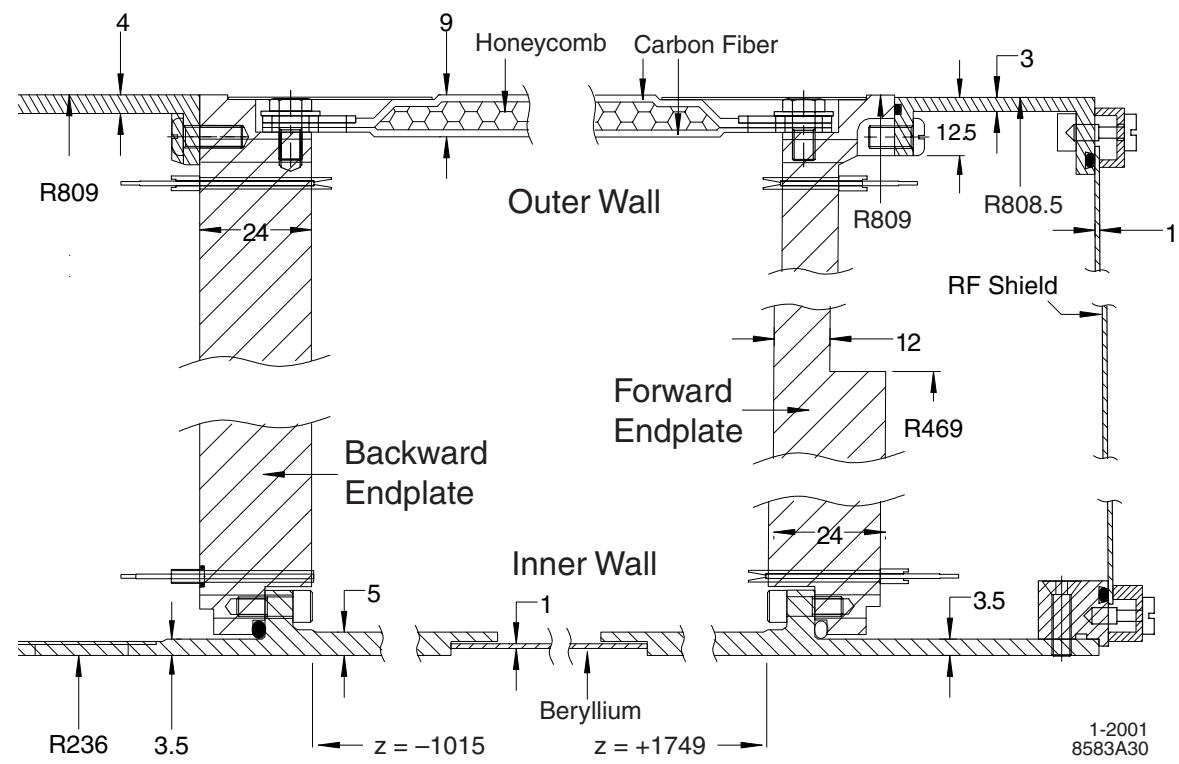

Figure 29. Details of the structural elements of the DCH. All components are made of aluminum, except for the $1 \mathrm{~mm}$-thick inner beryllium wall and the $9 \mathrm{~mm}$-thick outer composite wall.

tions. The achieved accuracy of the hole placement was $38 \mu \mathrm{m}$ for both sense and field wires, better than the specification by more than a factor of two. In addition, the diameters of the same sample of endplate holes were checked with precision gauge pins. All holes passed the diameter specification $\left(4.500 \pm_{0.000}^{0.025}\right.$ for sense wires and $2.500 \pm_{0.00}^{0.025}$ for the field and guard wires).

The inner cylindrical wall of the $\mathrm{DCH}$, which carries $40 \%$ of the wire load, was made from five sections, a central $1 \mathrm{~mm}$-thick beryllium tube with two aluminum extensions which were in turn electron-beam welded to two aluminum end flanges to form a $3 \mathrm{~m}$-long cylindrical part. The central section was made from three $120^{\circ} \mathrm{seg}$ ments of rolled and brazed beryllium. The end flanges have precision surfaces onto which the endplates were mounted. These surfaces set the angles of the two endplates with respect to the axis and significantly constrain the concentricity of the tube. The inner cylinder also provides a substantial rf shield down to the PEP-II bunchgap frequency of $136 \mathrm{kHz}$.

The outer wall bears $60 \%$ of axial wire load between the endplates. To simplify its installa- tion, this external wall was constructed from two half-cylinders with longitudinal and circumferential joints. The gas and electrical seals for these joints were made up in situ. The main structural element consists of two $1.6 \mathrm{~mm}$-thick $\left(0.006 X_{0}\right)$ carbon fiber skins laminated to a $6 \mathrm{~mm}$-thick honeycomb core. The outer shell is capable of withstanding a differential pressure of $30 \mathrm{mbar}$ and temperature variations as large as $\pm 20^{\circ} \mathrm{C}$, conditions that could be encountered during shipping or installation. Aluminum foil, $25 \mu \mathrm{m}$-thick on the inside surface and $100 \mu \mathrm{m}$ on the outside, are in good electrical contact with the endplates, thereby completing the rf shield for the chamber.

The total thickness of the DCH at normal incidence is $1.08 \% X_{0}$, of which the wires and gas mixture contribute $0.2 \% X_{0}$, and the inner wall $0.28 \% X_{0}$.

\subsubsection{Wire Feed-Throughs}

A total of five different types of feed-throughs were required for the chamber to accommodate the sense, field, and clearing field wires, as well as two different endplate thicknesses. The five types are illustrated in Figure 30. They incorporate crimp pins [43] of a simple design which fasten 
and precisely locate the wires. The choice of pin material (gold-plated copper for the signal wires and gold-plated aluminum for the field wires) and wall thickness in the crimp region was optimized to provide an allowable range of almost $150 \mu \mathrm{m}$ in crimp size, as a primary means for avoiding wire breakage.

Crimp pins were either press-fit into an insulator made from a single piece of injectionmolded thermoplastic reinforced with $30 \%$ silica glass fiber [44], or swaged into a copper jacket for the field wires. The plastic insulates the sense, guard, and clearing field wires from the electrically grounded endplates, while the metal jackets provide good ground contact for field wires $(<$ $0.1 \Omega$ ) on the backward endplate. The outer diameter of the field and clearing field feed-throughs was maintained at $2.000_{-0.025}^{+0.000} \mathrm{~mm}$ while the sense wire feed-through had a larger $\left(4.500_{-0.025}^{+0.000} \mathrm{~mm}\right)$ outer diameter and a longer body $(41.7 \mathrm{~mm})$. This choice provided both thicker insulating walls and a longer projection into the gas volume to better shield the HV from the grounded endplate.

\subsubsection{Assembly and Stringing}

Assembly of the chamber components and installation of the wires was carried out in a large clean room (Class 10,000) at TRIUMF in Vancouver. The wires were strung horizontally without the outer cylindrical shell in place. The endplates were mounted and aligned onto the inner cylinder which in turn was supported by a central shaft in a mobile fixture. The endplates were mounted on the inner cylinder at the inside rim and attached to support rings at the outside. These rings were connected by radial spiders to the central shaft of the stringing frame.

Two teams of two operators each worked in parallel as the wires were strung from the inner radius outward. The two teams were each assisted by an automated wire transporter [45]. A wire was attached to a needle which was inserted through one of the endplate hole, captured magnetically by one of the transporters, and then transported and inserted though the appropriate hole in the other endplate. The wire was then threaded through the feed-throughs, which were glued into the endplates, and the wire was ten-
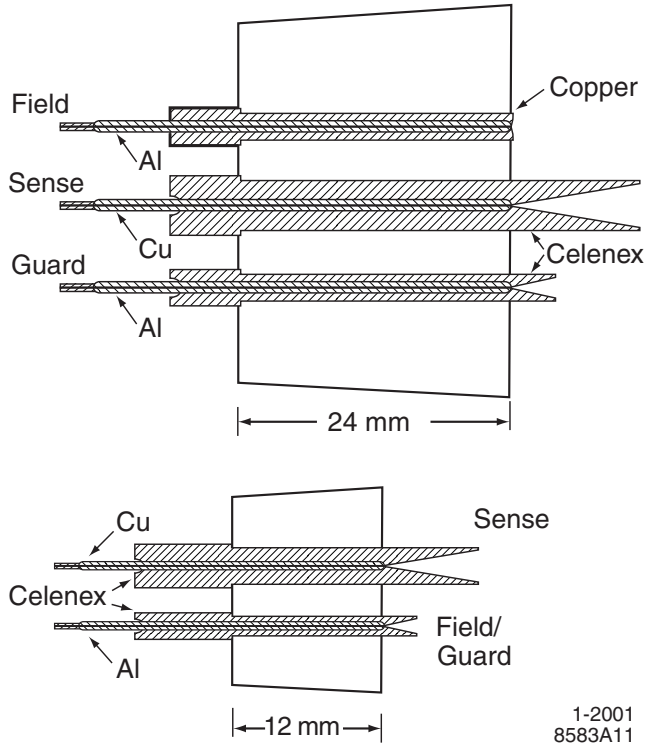

Figure 30. Design of the five DCH wire feedthroughs for the $24 \mathrm{~mm}$-thick endplates and the $12 \mathrm{~mm}$-thick endplate. The copper jacketed feedthrough is for grounded field wires, the other four are for sense wires ( $4.5 \mathrm{~mm}$ diameter), and guard and clearing field wires $(2.5 \mathrm{~mm}$ diameter $)$, all made from a Celenex insulator surrounding the crimp pins.

sioned and crimped. The automated wire transporters were largely built from industrial components, employing commercial software and hardware. The semi-automatic stringing procedure ensured the correct hole selection, accelerated the stringing rate and greatly improved the cleanliness and quality of the stringing process. The installation of a total of 28,768 wires was completed in less than 15 weeks.

\subsection{Drift Cells}

\subsubsection{Layer Arrangement}

The DCH consists of a total of 7,104 small drift cells, arranged in 40 cylindrical layers. The layers are grouped by four into ten superlayers, with the same wire orientation and equal numbers of cells in each layer of a superlayer. Sequential layers are staggered by half a cell. This arrangement enables local segment finding and left-right ambiguity resolution within a superlayer, even if one 
out of four signals is missing. The stereo angles of the superlayers alternate between axial (A) and stereo $(\mathrm{U}, \mathrm{V})$ pairs, in the order AUVAUVAUVA, as shown in Figure 31. The stereo angles vary between $\pm 45 \mathrm{mrad}$ and $\pm 76 \mathrm{mrad}$; they have been chosen such that the drilling patterns are identical for the two endplates. The hole pattern has a 16-fold azimuthal symmetry which is well suited to the modularity of the electronic readout and trigger system. Table 9 summarizes parameters for all superlayers.

\section{Table 9}

The DCH superlayer (SL) structure, specifying the number of cells per layer, radius of the innermost sense wire layer, the cell widths, and wire stereo angles, which vary over the four layers in a superlayer as indicated. The radii and widths are specified at the mid-length of the chamber.

\begin{tabular}{ccccc}
\hline SL & $\begin{array}{c}\text { \# of } \\
\text { Cells }\end{array}$ & $\begin{array}{c}\text { Radius } \\
(\mathrm{mm})\end{array}$ & $\begin{array}{c}\text { Width } \\
(\mathrm{mm})\end{array}$ & $\begin{array}{c}\text { Angle } \\
(\mathrm{mrad})\end{array}$ \\
\hline 1 & 96 & 260.4 & $17.0-19.4$ & 0 \\
2 & 112 & 312.4 & $17.5-19.5$ & $45-50$ \\
3 & 128 & 363.4 & $17.8-19.6$ & $-(52-57)$ \\
4 & 144 & 422.7 & $18.4-20.0$ & 0 \\
5 & 176 & 476.6 & $16.9-18.2$ & $56-60$ \\
6 & 192 & 526.1 & $17.2-18.3$ & $-(63-57)$ \\
7 & 208 & 585.4 & $17.7-18.8$ & 0 \\
8 & 224 & 636.7 & $17.8-18.8$ & $65-69$ \\
9 & 240 & 688.0 & $18.0-18.9$ & $-(72-76)$ \\
10 & 256 & 747.2 & $18.3-19.2$ & 0 \\
\hline
\end{tabular}

\subsubsection{Cell Design and Wires}

The drift cells are hexagonal in shape, $11.9 \mathrm{~mm}$ by approximately $19.0 \mathrm{~mm}$ along the radial and azimuthal directions, respectively. The hexagonal cell configuration is desirable because approximate circular symmetry can be achieved over a large portion of the cell. The choice of aspect ratio has the benefit of decreasing the number of wires and electronic channels, while allowing a 40-layer chamber in a confined radial space. Each cell consists of one sense wire surrounded by six field wires, as shown in Figure 31. The properties of the different types of gold-coated wires that

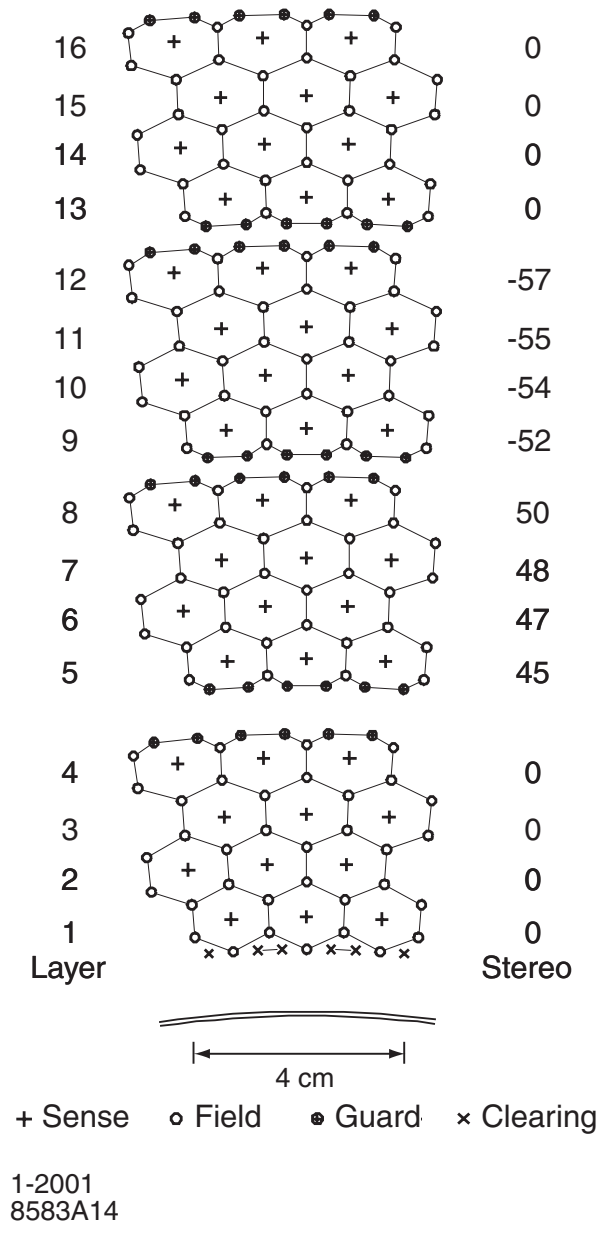

Figure 31. Schematic layout of drift cells for the four innermost superlayers. Lines have been added between field wires to aid in visualization of the cell boundaries. The numbers on the right side give the stereo angles (mrad) of sense wires in each layer. The $1 \mathrm{~mm}$-thick beryllium inner wall is shown inside of the first layer.

make up the drift cells are given in Table 10. The sense wires are made of tungsten-rhenium [46], $20 \mu \mathrm{m}$ in diameter and tensioned with a weight of $30 \mathrm{~g}$. The deflection due to gravity is $200 \mu \mathrm{m}$ at mid-length. Tungsten-rhenium has a substantially higher linear resistivity $(290 \Omega / \mathrm{m})$, compared to pure tungsten $(160 \Omega / \mathrm{m})$, but it is considerably stronger and has better surface quality. While the field wires are at ground potential, a 
positive high voltage is applied to the sense wires. An avalanche gain of approximately $5 \times 10^{4}$ is obtained at a typical operating voltage of $1960 \mathrm{~V}$ and a 80:20 helium:isobutane gas mixture.

Table 10

$\mathrm{DCH}$ wire specifications (all wires are gold plated).

\begin{tabular}{ccccc}
\hline Type & Material & $\begin{array}{c}\text { Diameter } \\
(\mu \mathrm{m})\end{array}$ & $\begin{array}{c}\text { Voltage } \\
(\mathrm{V})\end{array}$ & $\begin{array}{c}\text { Tension } \\
(\mathrm{g})\end{array}$ \\
\hline Sense & W-Re & 20 & 1960 & 30 \\
Field & $\mathrm{Al}$ & 120 & 0 & 155 \\
Guard & $\mathrm{Al}$ & 80 & 340 & 74 \\
Clearing & $\mathrm{Al}$ & 120 & 825 & 155 \\
\hline
\end{tabular}

The relatively low tension on the approximately $2.75 \mathrm{~m}$-long sense wires was chosen so that the aluminum field wires have matching gravitational sag and are tensioned well below the elastic limit. A simulation of the electrostatic forces shows that the cell configuration has no instability problems. At the nominal operating voltage of $1960 \mathrm{~V}$, the wires deflect by less then $60 \mu \mathrm{m}$.

The field wires [47] are tensioned with $155 \mathrm{~g}$ to match the gravitational sag of the sense wires to within $20 \mu \mathrm{m}$. This tension is less than onehalf the tensile yield strength of the aluminum wire. For cells at the inner or outer boundary of a superlayer, two guard wires are added to improve the electrostatic performance of the cell and to match the gain of the boundary cells to those of the cells in the inner layers. At the innermost boundary of layer 1 and the outermost boundary of layer 40, two clearing wires have been added per cell to collect charges created through photon conversions in the material of the walls.

\subsubsection{Drift Isochrones}

The calculated isochrones and drift paths for ions in adjacent cells of layer 3 and 4 of an axial superlayer are presented in Figure 32 . The isochrones are circular near the sense wires, but deviate greatly from circles near the field wires. Ions originating in the gap between superlayers are collected by cells in the edge layers after a delay of several $\mu \mathrm{s}$. These lagging ions do not affect

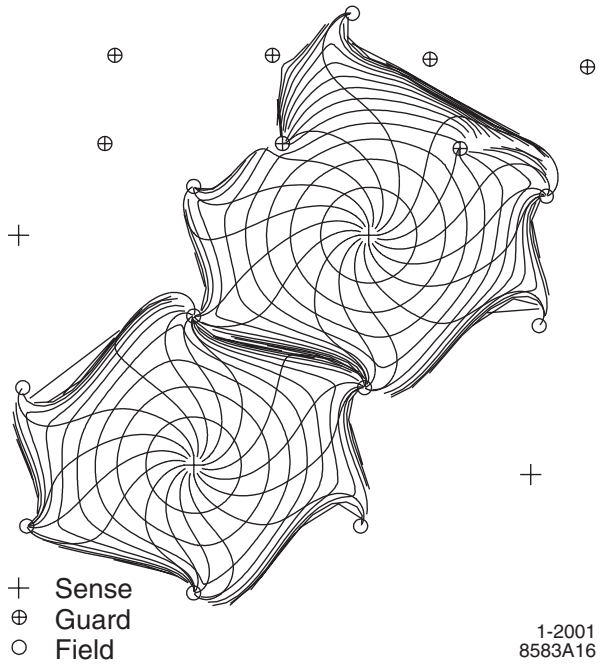

Figure 32. Drift cell isochrones, i.e., contours of equal drift times of ions in cells of layers 3 and 4 of an axial superlayer. The isochrones are spaced by $100 \mathrm{~ns}$. They are circular near the sense wires, but become irregular near the field wires, and extend into the gap between superlayers.

the drift times measurements, but they contribute to the $d E / d x$ measurement.

\subsubsection{Cross Talk}

A signal on one sense wire produces oppositelycharged signals on neighboring wires due to capacitive coupling. The cross talk is largest between adjacent cells of adjacent layers, ranging from $-0.5 \%$ at a superlayer boundary to $-2.7 \%$ for internal layers within superlayers. For adjacent cells in the same layer, the cross talk ranges from -0.8 to $-1.8 \%$, while for cells separated by two layers it is less than $0.5 \%$.

\subsection{Electronics}

6.4.1. Design Requirements and Overview The DCH electronic system is designed to provide a measurement of the drift time and the integrated charge, as well as a single bit to the trigger system [48] for every wire with a signal. In the 80:20 helium:isobutane gas mixture, there are on average some 22 primary and 44 total ionization clusters produced per $\mathrm{cm}$. The position of the primary ionization clusters is derived from timing of the leading edge of the amplified signal. 


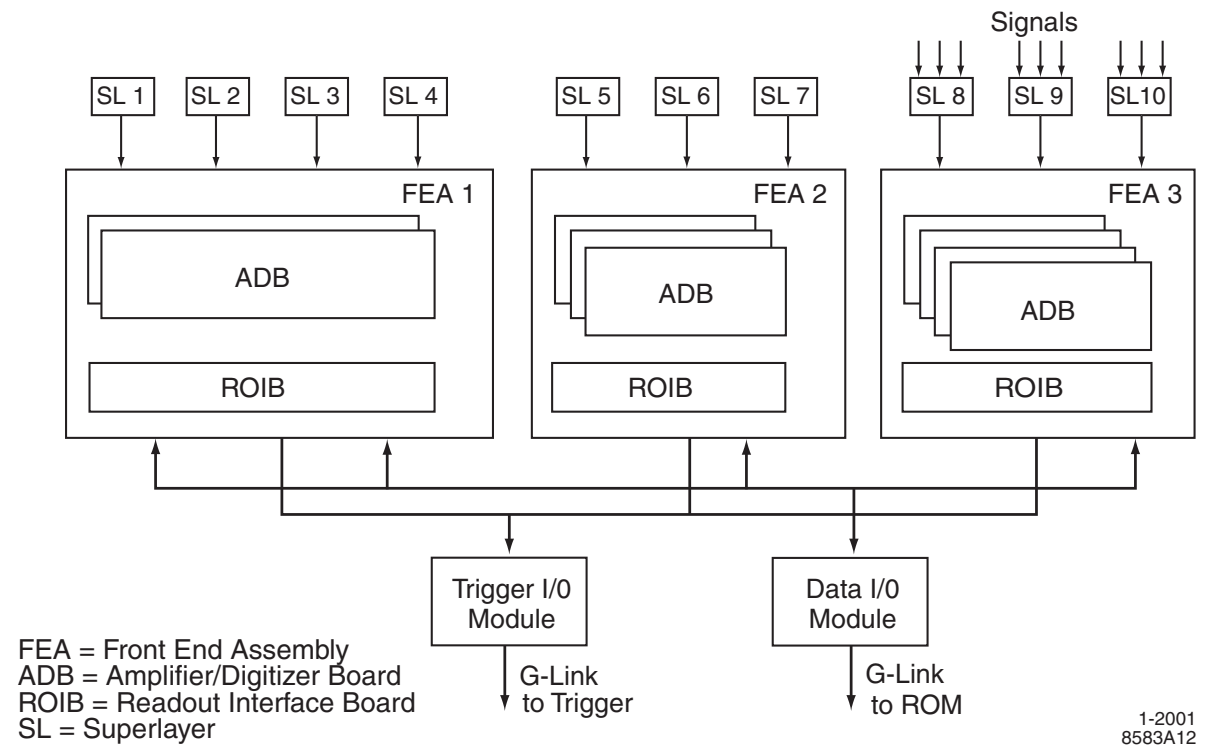

Figure 33. Block diagram for a $1 / 16^{\text {th }}$ wedge of the DCH readout system, showing logical organization of the three front-end assemblies and their connections to the trigger and data I/O modules

The design goal was to achieve a position resolution of $140 \mu \mathrm{m}$, averaged over the cells. To reduce the time jitter in the signal arrival and at the same time maintain a good signal-to-noise ratio, the signal threshold was set at about 2.5 primary electrons. For the $d E / d x$ measurement, a resolution of $7 \%$ was projected for a 40-layer chamber.

The small cell size and the difficult access through the DIRC strong support tube require a very high density of electronics components. As a consequence, a compact and highly modular design was chosen. The readout is installed in well shielded assemblies that are plugged into the endplate and are easily removable for maintenance.

A schematic overview of the DCH electronics is presented in Figure 33 [49]. The 16-fold azimuthal symmetry of the cell pattern is reflected in the readout segmentation. The DCH amplifier and digitizer electronics are installed in electronics front-end assemblies (FEAs) that are mounted directly onto the rear endplate. There are three FEAs in each of the 16 sectors. These sectors are separated by brass cooling bars that extend from the inner to the outer chamber walls. These bars provide mechanical support and water cooling channel for the FEAs. The assemblies connect to the sense wires through service boards, which route the signals and $\mathrm{HV}$ distribution. A readout interface board (ROIB) in each FEA organizes the readout of the digitized data. Data $\mathrm{I} / \mathrm{O}$ and trigger I/O modules multiplex serial data from the FEAs to high-speed optical fibers for transfer to the readout modules that are located in the electronics building.

\subsubsection{Service Boards}

Service boards provide the electrostatic potentials for signal, guard, and clearing wires, and pass signals and ground to the front-end readout electronics. A side view of a service board is shown in Figure 34. The HV board contains the HV buses and filtering, current limiting resistors, and blocking capacitors. Jumpers connect adjacent boards. The stored energy is minimized by using a $220 \mathrm{pF}$ HV blocking capacitors.

The signals are connected via series resistors to the upper signal board which contains the protection diodes and standard output connectors. Mounting posts, anchored into the rear endplate, also serve as ground connections. 


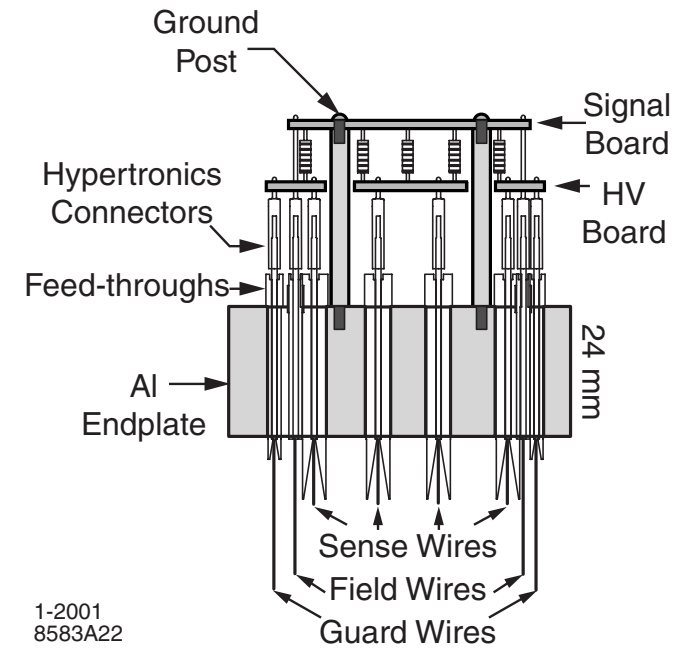

Figure 34. Side view of service boards showing two-tiered structure for DCH HV distribution and signal collection.

\subsubsection{Front-End Assemblies}

The FEAs plug into connectors on the back side of the service boards. These custom wedgeshaped crates are aluminum boxes that contain a ROIB and two, three, or four amplifier/digitizer boards (ADB) for superlayers $1-4,5-7$, and 8-10, respectively, as shown in Figure 35. The crates are mounted with good thermal contact to the water cooled radial support bars. The total heat load generated by the FEAs is $1.3 \mathrm{~kW}$.

The ADBs are built from basic building blocks consisting of two 4-channel amplifier ICs [50] feeding a single 8-channel digitizer custom ASIC [51]. The number of channels serviced by an ADB is 60,48 , or 45 , for the inner, middle, and outer FEA modules, respectively.

The custom amplifier IC receives the input signal from the sense wire and produces a discriminator output signal for the drift time measurement and a shaped analog signal for the $d E / d x$ measurement. Both outputs are fully differential. The discriminator has gain and bandwidth control, and a voltage controlled threshold. The analog circuit has integrator and gain control.

The custom digitizer IC incorporates a 4-bit TDC for time measurement and a 6 -bit $15 \mathrm{MHz}$ FADC to measure the total deposited charge.

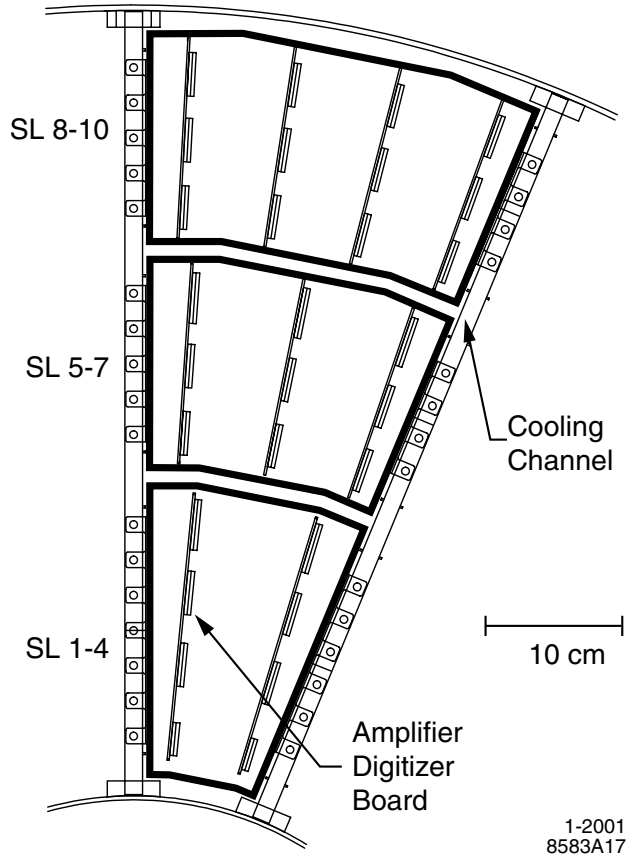

Figure 35. Layout of $1 / 16^{\text {th }}$ of the DCH rear endplate, showing three FEA boxes between water cooled channels.

The TDC is a phase-locked digital delay linear vernier on the sample clock of $15 \mathrm{MHz}$, which achieves a $1 \mathrm{~ns}$ precision for leading edge timing. The FADC design is based on a resistordivider comparator ladder that operates in bilinear mode to cover the full dynamic range. The digitized output signals are stored in a trigger latency buffer for $12.9 \mu \mathrm{s}$, after which a L1 Accept initiates the transfer of a $2.2 \mu$ s block of data to the readout buffer. In addition, trigger information is supplied for every channel, based either on the presence of a TDC hit during the sample period or FADC differential pulse height information, should a higher discriminator level be desirable.

The ROIB interprets FCTS commands to control the flow of data and trigger information. Data are moved to FIFOs on the ROIBs, and then to data and trigger I/O modules via $59.5 \mathrm{MHz}$ serial links. A total of four such links are required per $1 / 16^{t h}$ wedge, one for each of the outer two FEAs and two for the innermost of the FEA. Each 
data I/O module services all FEAs one quadrant and transmits the data to a single ROM via one optical fiber link. The trigger stream is first multiplexed onto a total of 30 serial lines per wedge for transmission to the trigger I/O module. Trigger data from two wedges of FEAs are then transmitted to the trigger system via three optical links. Thus, a total of 28 optical fibers, four for the data and 24 for the charged particle trigger, are required to transfer the $\mathrm{DCH}$ data to the readout.

\subsubsection{Data Acquisition}

The data stream is received and controlled by four BABAR standard readout modules. Drift chamber-specific feature extraction algorithms convert the raw FADC and TDC information into drift times, total charge, and a status word. The time and charge are corrected channel-by-channel for time offsets, pedestals, and gain constants. Based on measurements of the noise a threshold is typically $2-3$ electrons is applied to discriminate signals. These algorithms take about $1 \mu$ s per channel, and reduce the data volume by roughly a factor of four.

\subsubsection{High Voltage System}

The HV bias lines on the chamber are daisychained together so that each superlayer requires only four power supplies, except for superlayer 1 which has eight. The voltages are supplied to the sense, guard, and clearing wires by a CAEN SY527 HV mainframe [42], equipped with 24channel plug-in modules. The sense wires are supplied by $44 \mathrm{HV}$ channels providing up to $40 \mu \mathrm{A}$ of current each that can be monitored with a resolution of $0.1 \%$.

\subsection{Gas System}

The gas system has been designed to provide a stable 80:20 helium:isobutane mixture at a constant over pressure of 4 mbar [52]. The chamber volume is about $5.2 \mathrm{~m}^{3}$. Gas mixing and recirculation is controlled by precise mass flow controllers; the total flow is tuned to $15 \ell / \mathrm{min}$, of which $2.5 \ell / \mathrm{min}$ are fresh gas. During normal operation, the complete DCH gas volume is recirculated in six hours, and one full volume of fresh gas is added every 36 hours. The pressure in the $\mathrm{DCH}$ is measured by two independent pressure gauges, one of which is connected to a regulator controlling the speed of the compressor. The relative pressure in the chamber is controlled to better than \pm 0.05 mbar.

Oxygen is removed from the gas mixture using a palladium catalytic filter. The water content is maintained at $3500 \pm 200 \mathrm{ppm}$ by passing an adjustable fraction of the gas through a water bubbler. This relatively high level of water vapor is maintained to prevent electrical discharge. In addition to various sensors to monitor pressure, temperature, and flow at several points of the system, a small wire chamber with an ${ }^{55} \mathrm{Fe}$ source continuously monitors gain of the gas mixture.

\subsection{Calibrations and Monitoring 6.7. Electronics Calibration}

The front-end electronics (FEEs) are calibrated daily to determine the channel-by-channel correction constants and thresholds. Calibration pulses are produced internally and input to the preamplifier at a rate of about $160 \mathrm{~Hz}$. The calibration signals are processed in the ROM to minimize the data transfer and fully exploit the available processing power. The results are stored for subsequent feature extraction. The entire online calibration procedure takes less than two minutes.

\subsubsection{Environmental Monitoring}

The operating conditions of the DCH are monitored in realtime by a variety of sensors and read out by the detector-wide CAN bus system. These sensors monitor the flow rate, pressure, and gas mixture; the voltages and currents applied to the wires in the chamber; the voltages and currents distributed to the electronics from power supplies and regulators; instantaneous and cumulative radiation doses; temperature and humidity around the chamber electronics and in the equipment racks. Additional sensors monitor the atmosphere in and around the detector for excess isobutane, which could pose a flammability or explosive hazard in the event of a leak.

Many of the sensors are connected to hardware interlocks, which ensure that the chamber is automatically put into a safe state in response to an unsafe condition. All of these systems have per- 
formed reliably. In addition, automated software monitors raw data quality, chamber occupancies and efficiencies to sense variations in electronics performance that might indicate more subtle operational problems.

\subsubsection{Operational Experience}

The design of the DCH specifies a voltage of $1960 \mathrm{~V}$ on the sense wires to achieve the desired gain and resolution. The chamber voltage was lowered for part of the run to $1900 \mathrm{~V}$ out of concern for a small region of the chamber that was damaged during the commissioning phase by inadvertently applying $2 \mathrm{kV}$ to the guard wires. Wires in this region (10.4\% of superlayer 5 , and $4.2 \%$ of superlayer 6 ) were disconnected when continuous discharge was observed over extended periods of time.

\subsection{Performance}

The DCH was first operated with full magnetic field immediately after the installation into $B A B A R$. Cosmic ray data were recorded and extensive studies of the basic cell performance were performed to develop calibration algorithms for the time-to-distance and $d E / d x$ measurements. These algorithms were then implemented as described below for colliding beam data. Calibrations are monitored continuously to provide feedback to the operation; some time varying parameters are updated continuously as part of OPR. For charge particle tracking the DCH and SVT information is combined; the performance of the combined tracking system is described in Section 7 .

\subsubsection{Time-to-Distance Relation}

The precise relation between the measured drift time and drift distance is determined from samples of $e^{+} e^{-}$and $\mu^{+} \mu^{-}$events. For each signal, the drift distance is estimated by computing the distance of closest approach between the track and the wire. To avoid bias, the fit does not use the hit on the wire under consideration. The estimated drift distances and measured drift times are averaged over all wires in a layer, but the data are accumulated separately for tracks passing on the left of a sense wire and on the right. The timedistance relation is fit to a sixth-order Chebychev polynomial. An example of such a fit is shown in

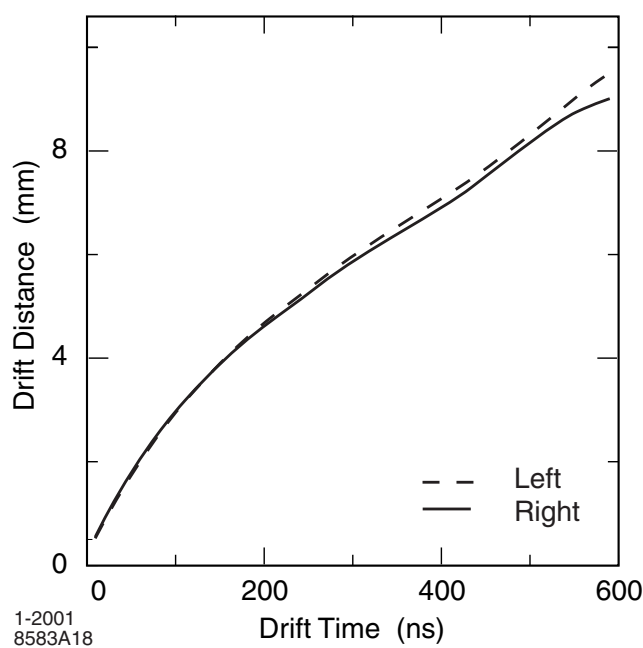

Figure 36. The drift time versus distance relation for left and right half of a cell. These functions are obtained from the data averaged over all cells in a single layer of the $\mathrm{DCH}$.

Figure 36 .

An additional correction is made for tracks with varying entrance angle into the drift cell. This angle is defined relative to the radial vector from the IP to the sense wire. The correction is applied as a scale factor to the drift distance and was determined layer-by-layer from a Garfield [53] simulation. The entrance angle correction is implemented as a tenth-order Chebychev polynomial of the drift distance, with coefficients which are functions of the entrance angle.

Figure 37 shows the position resolution as a function of the drift distance, separately for the left and the right side of the sense wire. The resolution is taken from Gaussian fits to the distributions of residuals obtained from unbiased track fits. The results are based on multi-hadron events, for data averaged over all cells in layer 18 .

\subsubsection{Charge Measurement}

The specific energy loss, $d E / d x$, for charged particles traversing the $\mathrm{DCH}$ is derived from measurement of total charge deposited in each drift cell. The charge collected per signal cell is measured as part of the feature extraction algorithm in the ROM. Individual measurements are corrected for gain variations, pedestal-subtracted 


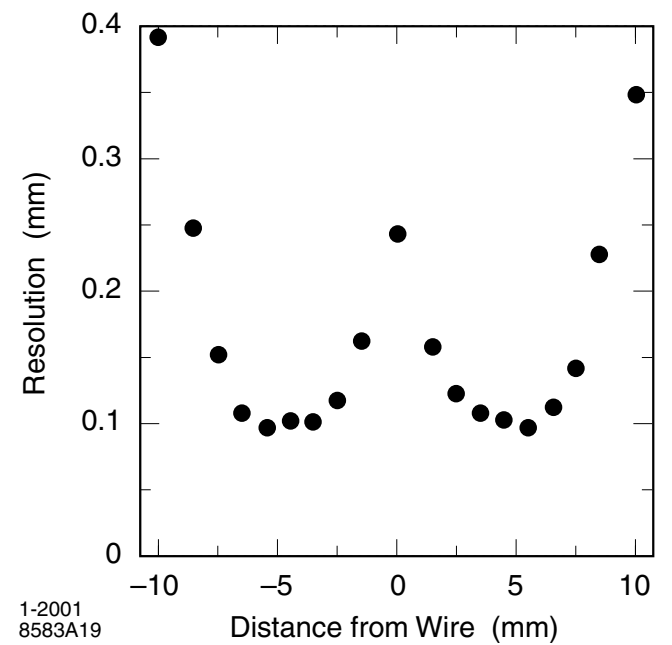

Figure 37. DCH position resolution as a function of the drift distance in layer 18 , for tracks on the left and right side of the sense wire. The data are averaged over all cells in the layer.

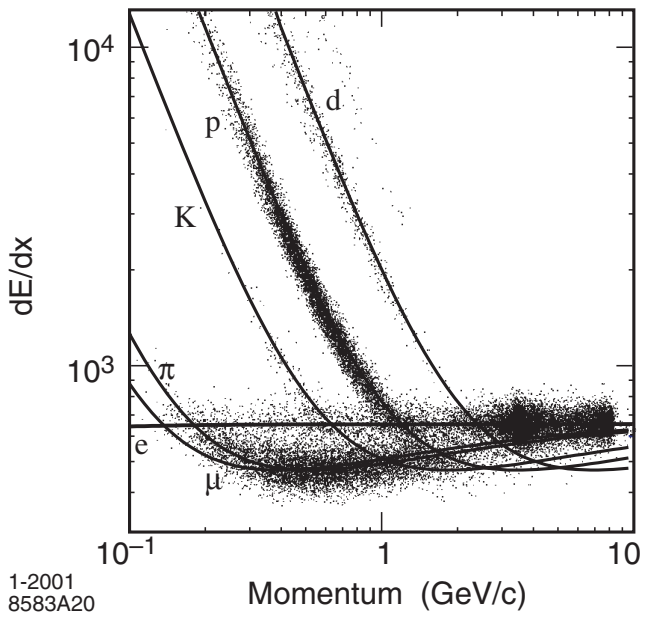

Figure 38. Measurement of $d E / d x$ in the DCH as a function of track momenta. The data include large samples of beam background triggers, as evident from the high rate of protons. The curves show the Bethe-Bloch predictions derived from selected control samples of particles of different masses. and integrated over a period of approximately $1.8 \mu \mathrm{s}$.

The specific energy loss per track is computed as a truncated mean from the lowest $80 \%$ of the individual $d E / d x$ measurements. Various corrections are applied to remove sources of bias that degrade the accuracy of the primary ionization measurement. These corrections include the following:

- changes in gas pressure and temperature, leading to $\pm 9 \%$ variation in $d E / d x$, corrected by a single overall multiplicative constant;

- differences in cell geometry and charge collection ( $\pm 8 \%$ variation), corrected by a set of multiplicative constants for each wire;

- signal saturation due to space charge buildup ( $\pm 11 \%$ variation $)$, corrected by a secondorder polynomial in the dip angle, $\lambda$, of the form $1 / \sqrt{\sin ^{2} \lambda+\text { const }}$;

- non-linearities in the most probable energy loss at large dip angles ( $\pm 2.5 \%$ variation), corrected with a fourth-order Chebychev polynomial as a function of $\lambda$; and

- variation of cell charge collection as a function entrance angle ( $\pm 2.5 \%$ variation $)$, corrected using a sixth-order Chebychev polynomial in the entrance angle.

The overall gas gain is updated continuously based on calibrations derived as part of prompt reconstruction of the colliding beam data; the remaining corrections are determined once for a given $\mathrm{HV}$ voltage setting and gas mixture.

Corrections applied at the single-cell level can be large compared to the single-cell $d E / d x$ resolution, but have only a modest impact on the average resolution of the ensemble of hits. Global corrections applied to all hits on a track are therefore the most important for the resolution.

Figure 38 shows the distribution of the corrected $d E / d x$ measurements as a function of track momenta. The superimposed Bethe-Bloch predictions for particles of different masses have been determined from selected control samples. 


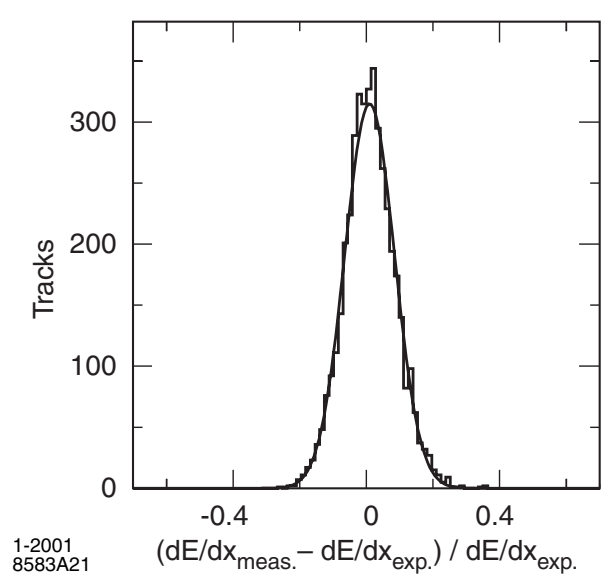

Figure 39. Difference between the measured and expected energy loss $d E / d x$ for $e^{ \pm}$from Bhabha scattering, measured in the $\mathrm{DCH}$ at an operating voltage of $1900 \mathrm{~V}$. The curve represents a Gaussian fit to the data with a resolution of $7.5 \%$.

The measured $d E / d x$ resolution for Bhabha events is shown in Figure 39. The rms resolution achieved to date is typically $7.5 \%$, limited by the number of samples and Landau fluctuations. This value is close to the expected resolution of $7 \%$. Further refinements and additional corrections are being considered to improve performance.

\subsection{Conclusions}

The DCH has been performing close to design expectations from the start of operations. With the exception of a small number of wires that were damaged by an unfortunate $\mathrm{HV}$ incident during the commissioning phase, all cells are fully operational. The DCH performance has proven very stable over time. The design goal for the intrinsic position and $d E / d x$ resolution have been met. Backgrounds are acceptable at present beam currents, but there is concern for rising occupancies and data acquisition capacity at the high end of the planned luminosity upgrades.

\section{Performance of the Charged Particle Tracking Systems}

Charged particle tracking has been studied with large samples of cosmic ray muons, $e^{+} e^{-}$, $\mu^{+} \mu^{-}$, and $\tau^{+} \tau^{-}$events, as well as multi-hadrons. At this time, these studies are far from complete and the results represent the current status. In particular, many issues related to the intrinsic alignment of the SVT and the $\mathrm{DCH}$, the variation with time of the relative alignment of the SVT and the DCH, and movement of the beam position relative to $B A B A R$ remain under study.

\subsection{Track Reconstruction}

The reconstruction of charged particle tracks relies on data from both tracking systems, the SVT and the DCH. Charged tracks are defined by five parameters $\left(d_{0}, \phi_{0}, \omega, z_{0}, \tan \lambda\right)$ and their associated error matrix. These parameters are measured at the point of closest approach to the $z$-axis; $d_{0}$ and $z_{0}$ are the distances of this point from the origin of the coordinate system in the $x-y$ plane and along the $z$-axis, respectively. The angle $\phi_{0}$ is the azimuth of the track, $\lambda$ the dip angle relative to the transverse plane, and $\omega=1 / p_{t}$ is its curvature. $d_{0}$ and $\omega$ are signed variables; their sign depends on the charge of the track. The track finding and the fitting procedures make use of Kalman filter algorithm [54] that takes into account the detailed distribution of material in the detector and the full map of the magnetic field.

The offline charged particle track reconstruction builds on information available from the L3 trigger and tracking algorithm. It begins with an improvement of the event start time $t_{0}$, obtained from a fit to the parameters $d_{0}, \phi_{0}$, and $t_{0}$ based on the four-hit track segments in the DCH superlayers. Next, tracks are selected by performing helix fits to the hits found by the L3 track finding algorithm. A search for additional hits in the DCH that may belong on these tracks is performed, while $t_{0}$ is further improved by using only hits associated with tracks. Two more sophisticated tracking procedures are applied which are designed to find tracks that either do not pass through the entire $\mathrm{DCH}$ or do not originate from the IP. These algorithms primarily use track segments that have not already been assigned to other tracks, and thus benefit from a progressively cleaner tracking environment with a constantly improving $t_{0}$. At the end of this process, tracks are again fit using a Kalman filter method. 

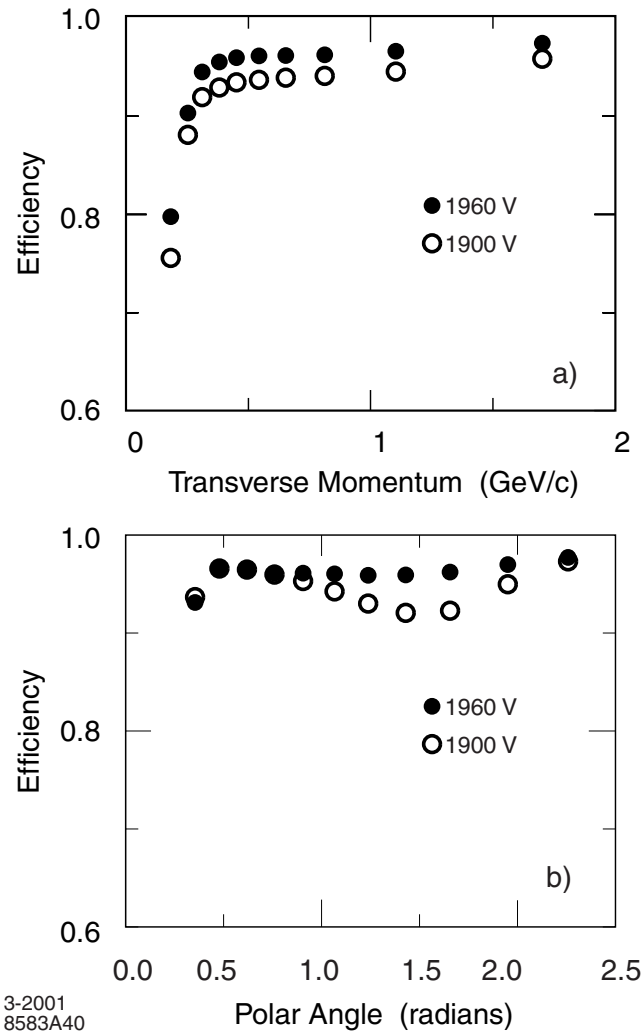

Figure 40. The track reconstruction efficiency in the DCH at operating voltages of $1900 \mathrm{~V}$ and $1960 \mathrm{~V}$, as a function of a) transverse momentum, and b) polar angle. The efficiency is measured in multi-hadron events as the fraction of all tracks detected in the SVT for which the DCH portion is also reconstructed.

The resulting tracks are then extrapolated into the SVT, and SVT track segments are added, provided they are consistent with the expected error in the extrapolation through the intervening material and inhomogeneous magnetic field. Among the possible SVT segments, those with the smallest residuals and the largest number of SVT layers are retained and a Kalman fit is performed to the full set of DCH and SVT hits.

Any remaining SVT hits are then passed to two complementary standalone track finding algorithms. The first reconstructs tracks starting with triplets of space points (matched $\phi$ and $z$ hits) in layers 1,3 , and 5 of the SVT, and adding

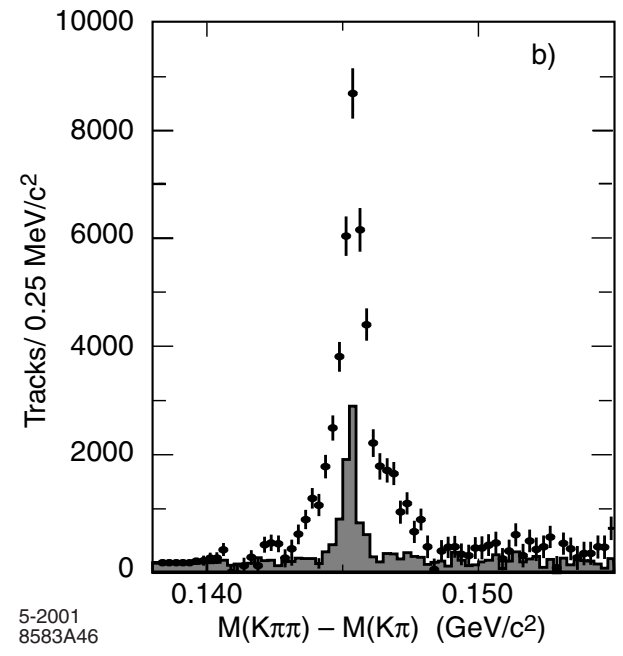

Figure 41. Reconstruction of low momentum tracks: the mass difference, $\Delta M=$ $M\left(K^{-} \pi^{+} \pi^{+}\right)-M\left(K^{-} \pi^{+}\right)$, both for all detected events (data points) and for events in which the low momentum pion is reconstructed both in the SVT and DCH (histogram). Backgrounds from combinatorics and fake tracks, as well as nonresonant data have been subtracted.

consistent space points from the other layers. A minimum of four space points are required to form a good track. This algorithm is efficient over a wide range of $d_{0}$ and $z_{0}$ values. The second algorithm starts with circle trajectories from $\phi$ hits and then adds $z$ hits to form helices. This algorithm is less sensitive to large combinatorics and to missing $z$ information for some of the SVT modules.

Finally, an attempt is made to combine tracks that are only found by one of the two tracking systems and thus recover tracks scattered in the material of the support tube.

\subsection{Tracking Efficiency}

The efficiency for reconstructing tracks in the DCH has been measured as a function of transverse momentum, polar and azimuthal angles in multi-track events. These measurements rely on specific final states and exploit the fact that the track reconstruction can be performed independently in the SVT and the DCH. 


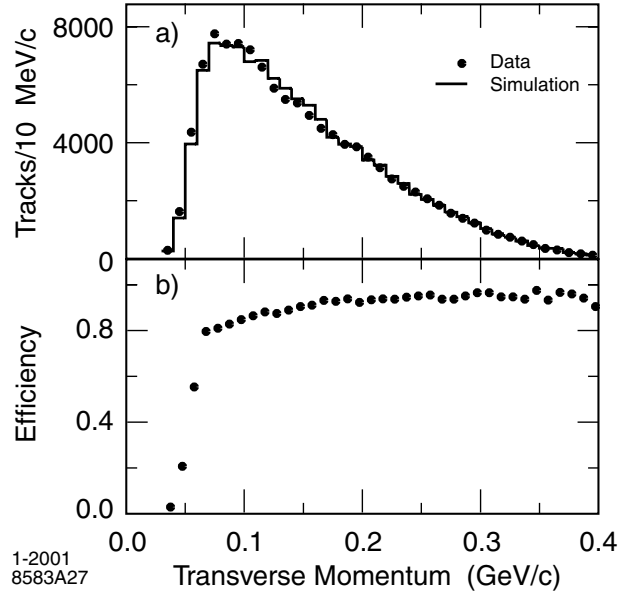

Figure 42. Monte Carlo studies of low momentum tracks in the SVT: a) comparison of data (contributions from combinatoric background and non$B \bar{B}$ events have been subtracted) with simulation of the transverse momentum spectrum of pions from $D^{*+} \rightarrow D^{0} \pi^{+}$in $B \bar{B}$ events, and b) efficiency for slow pion detection derived from simulated events.

The absolute DCH tracking efficiency is determined as the ratio of the number of reconstructed $\mathrm{DCH}$ tracks to the number of tracks detected in the SVT, with the requirement that they fall within the acceptance of the DCH. Such studies have been performed for different samples of multi-hadron events. Figure 40 shows the result of one such study for the two voltage settings. The measurement errors are dominated by the uncertainty in the correction for fake tracks in the SVT. At the design voltage of $1960 \mathrm{~V}$, the efficiency averages $98 \pm 1 \%$ per track above $200 \mathrm{MeV} / c$ and polar angle $\theta>500 \mathrm{mrad}$. The data recorded at $1900 \mathrm{~V}$ show a reduction in efficiency by about $5 \%$ for tracks at close to normal incidence, indicating that the cells are not fully efficient at this voltage.

The standalone SVT tracking algorithms have a high efficiency for tracks with low transverse momentum. This feature is important for the detection of $D^{*}$ decays. To study this efficiency, decays $D^{*+} \rightarrow D^{0} \pi^{+}$are selected by reconstructing events of the type $\bar{B} \rightarrow D^{*+} X$ followed by $D^{*+} \rightarrow D^{0} \pi^{+} \rightarrow K^{-} \pi^{+} \pi^{+}$. For the majority of these low momentum pions the momentum resolution is limited by multiple scattering, but the production angle can be determined from the signals in innermost layers of the SVT. Figure 41 shows the mass difference $\Delta M=M\left(K^{-} \pi^{+} \pi^{+}\right)-M\left(K^{-} \pi^{+}\right)$, for the total sample and the subsample of events in which the slow pion has been reconstructed in both the SVT and the DCH. The difference in these two distributions demonstrates the contribution from SVT standalone tracking, both in terms of the gain of signal events and of resolution. The gain in efficiency is mostly at very low momenta, and the resolution is impacted by multiple scattering and limited track length of the slow pions. To derive an estimate of the tracking efficiency for these low momentum tracks, a detailed Monte Carlo simulation was performed. Specifically, the pion spectrum was derived from simulation of the inclusive $D^{*}$ production in $B \bar{B}$ events, and the Monte Carlo events were selected in the same way as the data. A comparison of the detected slow pion spectrum with the Monte Carlo prediction is presented in Figure 42. Based on this very good agreement, the detection efficiency has been derived from the Monte Carlo simulation. The SVT significantly extends the capability of the charged particle detection down to transverse momenta of $\sim 50 \mathrm{MeV} / c$.

\subsection{Track Parameter Resolutions}

The resolution in the five track parameters is monitored in OPR using $e^{+} e^{-}$and $\mu^{+} \mu^{-}$pair events. It is further investigated offline for tracks in multi-hadron events and cosmic ray muons.

Cosmic rays that are recorded during normal data-taking offer a simple way of studying the track parameter resolution. The upper and lower halves of the cosmic ray tracks traversing the DCH and the SVT are fit as two separate tracks, and the resolution is derived from the difference of the measured parameters for the two track halves. To assure that the tracks pass close to the beam interaction point, cuts are applied on the $d_{0}, z_{0}$, and $\tan \lambda$. The results of this comparison for the coordinates of the point of closest approach and the angles are shown in Figure 43 for tracks with momenta above $p_{t}$ of $3 \mathrm{GeV} / c$. The distributions 


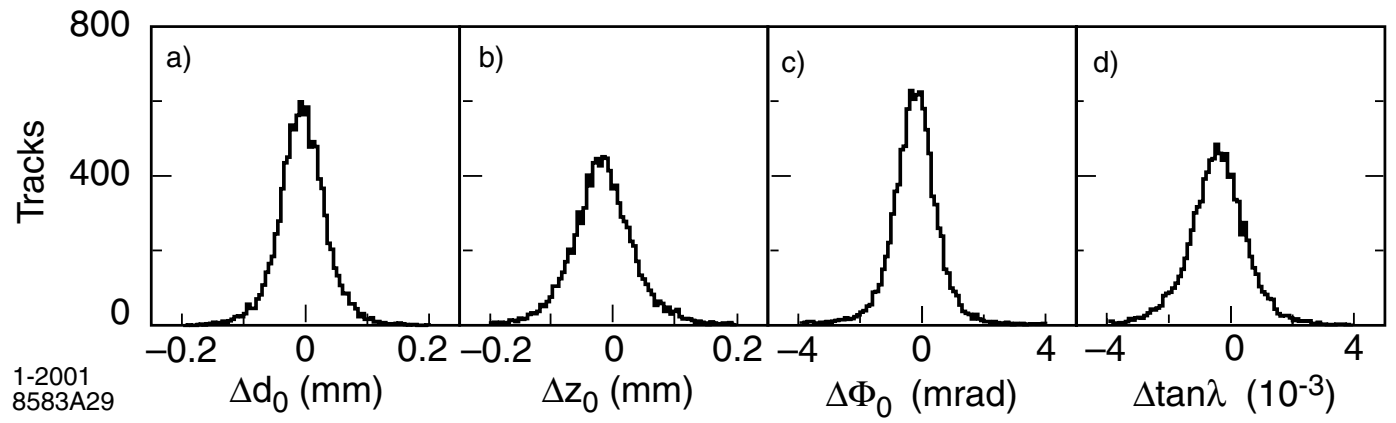

Figure 43. Measurements of the differences between the fitted track parameters of the two halves of cosmic ray muons, with transverse momenta above $3 \mathrm{GeV} / c$, a) $\Delta d_{0}$, b) $\Delta z_{0}$, c) $\Delta \phi_{0}$, and d) $\Delta \tan \lambda$.

are symmetric; the non-Gaussian tails are small. The distributions for the differences in $z_{0}$ and $\tan \lambda$ show a clear offset, attributed to residual problems with the internal alignment of the SVT. Based on the full width at half maximum of these distributions the resolutions for single tracks can be parametized as

$$
\begin{array}{ll}
\sigma_{d_{0}}=23 \mu \mathrm{m} & \sigma_{\phi_{0}}=0.43 \mathrm{mrad} \\
\sigma_{z_{0}}=29 \mu \mathrm{m} & \sigma_{\tan \lambda}=0.53 \cdot 10^{-3}
\end{array} .
$$

The dependence of the resolution in $d_{0}$ and $z_{0}$ on the transverse momentum $p_{t}$ is presented in Figure 44. The measurement is based on tracks in multi-hadron events. The resolution is determined from the width of the distribution of the difference between the measured parameters, $d_{0}$ and $z_{0}$, and the coordinates of the vertex reconstructed from the remaining tracks in the event. These distributions peak at zero, but have a tail for positive values due to the effect of particle decays. Consequently, only the negative part of the distributions reflects the measurement error and is used to extract the resolution. Event shape cuts and a cut on the $\chi^{2}$ of the vertex fit are applied to reduce the effect of weak decays on this measurement. The contribution from the vertex errors are removed from the measured resolutions in quadrature. The $d_{0}$ and $z_{0}$ resolutions so measured are about $25 \mu \mathrm{m}$ and $40 \mu \mathrm{m}$ respectively at $p_{t}=3 \mathrm{GeV} / c$. These values agree well with expectations, and are also in reasonable agreement with the results obtained from cosmic rays.

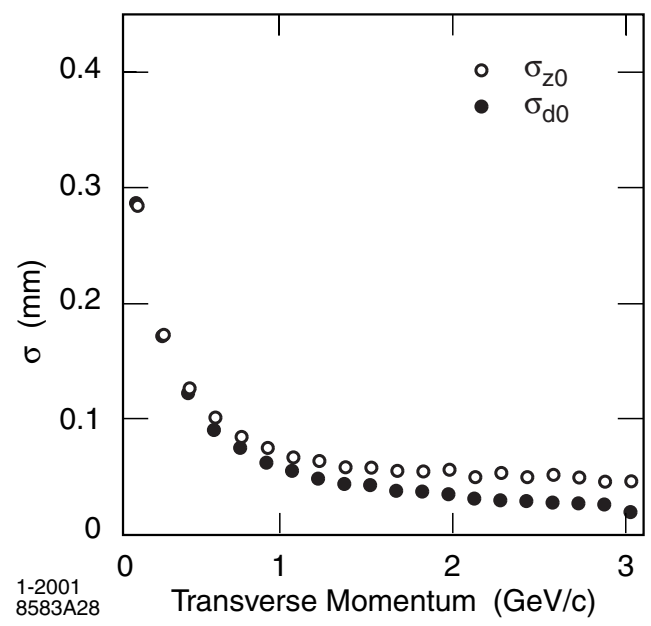

Figure 44. Resolution in the parameters $d_{0}$ and $z_{0}$ for tracks in multi-hadron events as a function of the transverse momentum. The data are corrected for the effects of particle decays and vertexing errors.

Figure 45 shows the estimated error in the measurement of the difference along the $z$-axis between the vertices of the two neutral $B$ mesons, one of them is fully reconstructed, the other serves as a flavor tag. The rms width of $190 \mu \mathrm{m}$ is dominated by the reconstruction of the tagging $B$ vertex, the rms resolution for the fully reconstructed $B$ meson is $70 \mu \mathrm{m}$. The data meet the design expectation [2].

While the position and angle measurements near the IP are dominated by the SVT measure- 


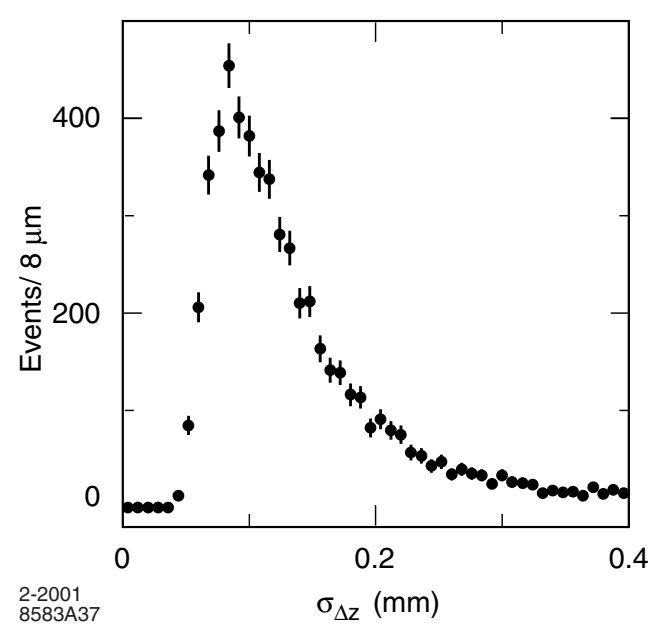

Figure 45. Distribution of the error on the difference $\Delta z$ between the $B$ meson vertices for a sample of events in which one $B^{0}$ is fully reconstructed.

ments, the DCH contributes primarily to the $p_{t}$ measurement. Figure 46 shows the resolution in the transverse momentum derived from cosmic muons. The data are well represented by a linear function,

$\sigma_{p_{t}} / p_{t}=(0.13 \pm 0.01) \% \cdot p_{t}+(0.45 \pm 0.03) \%$,

where the transverse momentum $p_{t}$ is measured in $\mathrm{GeV} / c$. These values for the resolution parameters are very close to the initial estimates and can be reproduced by Monte Carlo simulations. More sophisticated treatment of the DCH timeto-distance relations and overall resolution function are presently under study.

Figure 47 shows the mass resolution for $\mathrm{J} / \psi$ mesons reconstructed in the $\mu^{+} \mu^{-}$final state, averaged over all data currently available. The reconstructed peak is centered $0.05 \%$ below the expected value, this difference is attributed to the remaining inaccuracies in the SVT and DCH alignment and in the magnetic field parameterization. The observed mass resolution differs by $15 \%$ for data recorded at the two DCH HV settings, it is $13.0 \pm 0.3 \mathrm{MeV} / c^{2}$ and $11.4 \pm 0.3 \mathrm{MeV} / c^{2}$ at $1900 \mathrm{~V}$ and $1960 \mathrm{~V}$, respectively.

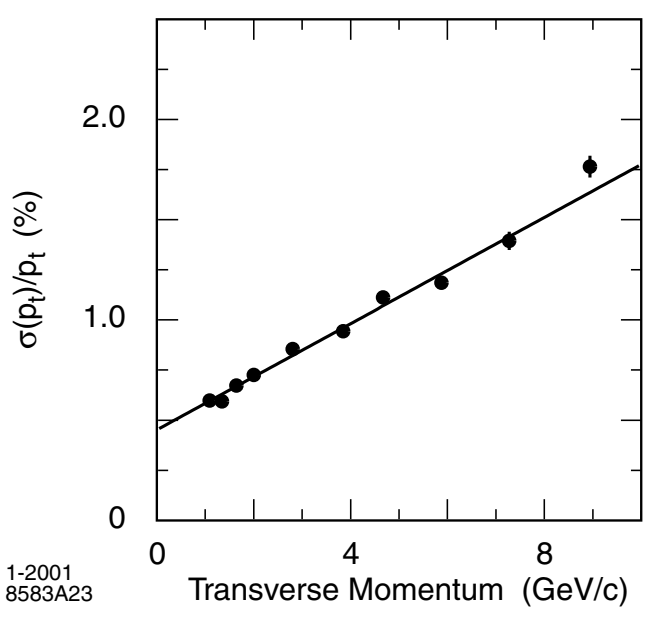

Figure 46. Resolution in the transverse momentum $p_{\mathrm{t}}$ determined from cosmic ray muons traversing the DCH and SVT.

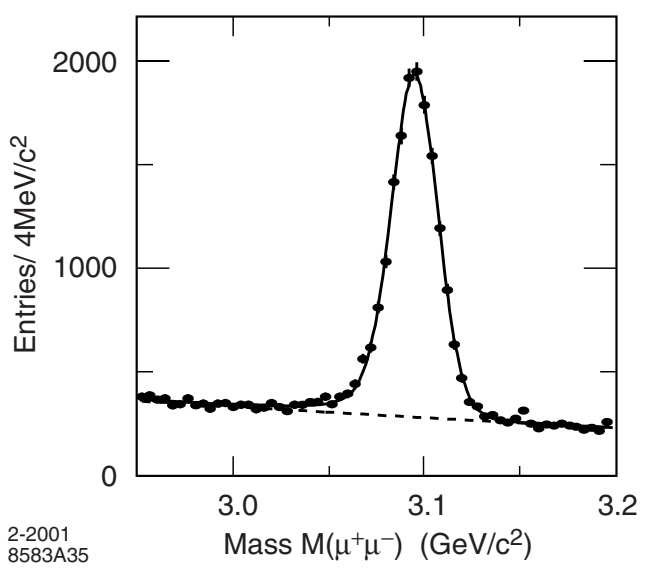

Figure 47. Reconstruction of the decay $J / \psi \rightarrow$ $\mu^{+} \mu^{-}$in selected $B \bar{B}$ events.

\subsection{Summary}

The two tracking devices, the SVT and DCH, have been performing close to design expectations from the start of operations. Studies of track resolution at lower momenta and as a function of polar and azimuthal angles are still under way. Likewise, the position and angular resolution at 
the entrance to the DIRC or EMC are still being studied. Such measurements are very sensitive to internal alignment of the SVT and relative placement of the SVT and the DCH. A better understanding will not only reduce the mass resolution for the reconstruction of exclusive states, it will also be important for improvement of the performance of the DIRC.

\section{DIRC}

\subsection{Purpose and Design Requirements}

The study of $C P$-violation requires the ability to tag the flavor of one of the $B$ mesons via the cascade decay $b \rightarrow c \rightarrow s$, while fully reconstructing the second $\mathrm{B}$ decay. The momenta of the kaons used for flavor tagging extend up to about $2 \mathrm{GeV} / c$, with most of them below 1 $\mathrm{GeV} / c$. On the other hand, pions and kaons from the rare two-body decays $B^{0} \rightarrow \pi^{+} \pi^{-}$and $B^{0} \rightarrow K^{+} \pi^{-}$must be well-separated. They have momenta between 1.7 and $4.2 \mathrm{GeV} / c$ with a strong momentum-polar angle correlation of the tracks (higher momenta occur at more forward angles because of the c.m. system boost) [4].

The Particle Identification (PID) system should be thin and uniform in terms of radiation lengths (to minimize degradation of the calorimeter energy resolution) and small in the radial dimension to reduce the volume, and hence, the cost of the calorimeter. Finally, for operation at high luminosity, the PID system needs fast signal response, and should be able to tolerate high backgrounds.

The PID system being used in BABAR is a new kind of ring-imaging Cherenkov detector called the DIRC [56] (the acronym DIRC stands for Detector of Internally Reflected Cherenkov light). It is expected to be able to provide $\pi / K$ separation of $\sim 4 \sigma$ or greater, for all tracks from $B$-meson decays from the pion Cherenkov threshold up to $4.2 \mathrm{GeV} / \mathrm{c}$. PID below $700 \mathrm{MeV} / \mathrm{c}$ relies primarily on the $d E / d x$ measurements in the $\mathrm{DCH}$ and SVT.

\subsection{DIRC Concept}

The DIRC is based on the principle that the magnitudes of angles are maintained upon re- flection from a flat surface. Figure 48 shows a schematic of the DIRC geometry that illustrates the principles of light production, transport, and imaging. The radiator material of the DIRC is synthetic, fused silica in the form of long, thin bars with rectangular cross section. These bars serve both as radiators and as light pipes for the portion of the light trapped in the radiator by total internal reflection. Fused, synthetic silica (Spectrosil [57]) is chosen because of its resistance to ionizing radiation, its long attenuation length, large index of refraction, low chromatic dispersion within the wavelength acceptance of the DIRC, and because it allows an excellent optical finish on the surfaces of the bars [58].

In the following, the variable $\theta_{c}$ is used to designate the Cherenkov angle, $\phi_{c}$ denotes the azimuthal angle of a Cherenkov photon around the track direction, and $n$ represents the mean index of refraction of fused silica $(n=1.473)$, with the familiar relation $\cos \theta_{c}=1 / n \beta(\beta=v / c, v=$ velocity of the particle, $c=$ velocity of light).

For particles with $\beta \approx 1$, some photons will always lie within the total internal reflection limit, and will be transported to either one or both ends of the bar, depending on the particle incident angle. To avoid instrumenting both ends of the bar with photon detectors, a mirror is placed at the forward end, perpendicular to the bar axis, to reflect incident photons to the backward, instrumented end.

Once photons arrive at the instrumented end, most of them emerge into a water-filled expansion region, called the standoff box. A fused silica wedge at the exit of the bar reflects photons at large angles relative to the bar axis. It thereby reduces the size of the required detection surface and recovers those photons that would otherwise be lost due to internal reflection at the fused silica/water interface. The photons are detected by an array of densely packed photomultiplier tubes (PMTs), each surrounded by reflecting light catcher cones [59] to capture light which would otherwise miss the active area of the PMT. The PMTs are placed at a distance of about $1.2 \mathrm{~m}$ from the bar end. The expected Cherenkov light pattern at this surface is essentially a conic section, where the cone opening- 


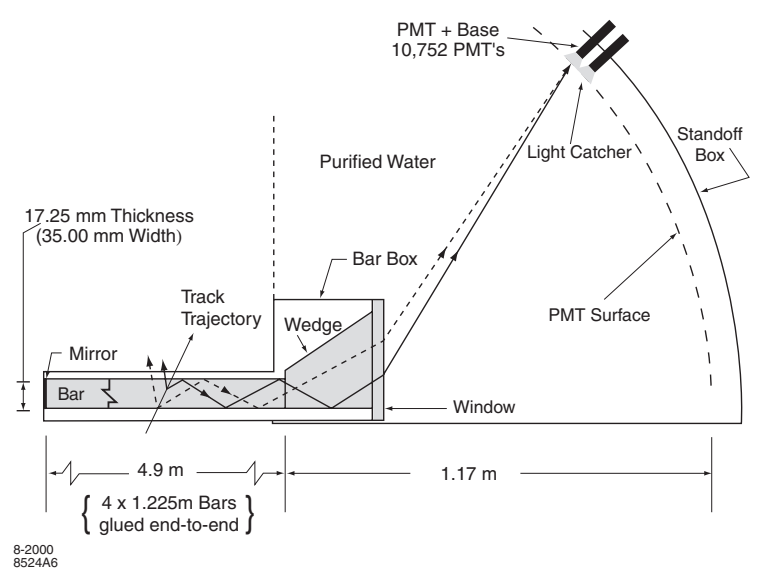

Figure 48. Schematics of the DIRC fused silica radiator bar and imaging region. Not shown is a $6 \mathrm{mrad}$ angle on the bottom surface of the wedge (see text).

angle is the Cherenkov production angle modified by refraction at the exit from the fused silica window.

The DIRC is intrinsically a three-dimensional imaging device, using the position and arrival time of the PMT signals. Photons generated in a bar are focused onto the phototube detection surface via a "pinhole" defined by the exit aperture of the bar. In order to associate the photon signals with a track traversing a bar, the vector pointing from the center of the bar end to the center of each PMT is taken as a measure of the photon propagation angles $\alpha_{x}, \alpha_{y}$, and $\alpha_{z}$. Since the track position and angles are known from the tracking system, the three $\alpha$ angles can be used to determine the two Cherenkov angles $\theta_{c}$ and $\phi_{c}$. In addition, the arrival time of the signal provides an independent measurement of the propagation of the photon, and can be related to the propagation angles $\alpha$. This over-constraint on the angles and the signal timing are particularly useful in dealing with ambiguities in the signal association (see Section 8.6.1) and high background rates.

\subsection{Mechanical Design and Physical Description}

The DIRC bars are arranged in a 12-sided polygonal barrel. Because of the beam energy asymmetry, particles are produced preferentially forward in the detector. To minimize interference with other detector systems in the forward region, the DIRC photon detector is placed at the backward end.

The principal components of the DIRC are shown schematically in Figure 49. The bars are placed into 12 hermetically sealed containers, called bar boxes, made of very thin aluminumhexcel panels. Each bar box, shown in Figure 50, contains 12 bars, for a total of 144 bars. Within a bar box the 12 bars are optically isolated by a $\sim 150 \mu \mathrm{m}$ air gap between neighboring bars, enforced by custom shims made from aluminum foil.

The bars are $17 \mathrm{~mm}$-thick, $35 \mathrm{~mm}$-wide, and 4.9 m-long. Each bar is assembled from four $1.225 \mathrm{~m}$ pieces that are glued end-to-end; this length is the longest high-quality bar currently obtainable [58, $60]$.

The bars are supported at $600 \mathrm{~mm}$ intervals by small nylon buttons for optical isolation from the bar box. Each bar has a fused silica wedge glued to it at the readout end. The wedge, made of the same material as the bar, is $91 \mathrm{~mm}$-long with very nearly the same width as the bars $(33 \mathrm{~mm})$ and a trapezoidal profile $(27 \mathrm{~mm}$-high at bar end, and $79 \mathrm{~mm}$ at the light exit end). The bottom of the wedge (see Figure 48$)$ has a slight ( $~ 6 \mathrm{mrad})$ upward slope to minimize the displacement of the downward reflected image due to the finite bar thickness. The twelve wedges in a bar box are glued to a common $10 \mathrm{~mm}$-thick fused silica window, that provides the interface and seal to the purified water in the standoff box.

The mechanical support of the DIRC, shown in Figure 49, is cantilevered from the steel of the IFR. The Strong Support Tube (SST) is a steel cylinder located inside the end doors of the IFR and provides the basic support for the entire DIRC. In turn, the SST is supported by a steel support gusset that fixes it to the barrel magnet steel. It also minimizes the magnetic flux gap caused by the DIRC bars extending through the flux return, and supports the axial load of the inner magnetic plug surrounding the beam in this region.

The bar boxes are supported in the active region by an aluminum tube, the Central Support 


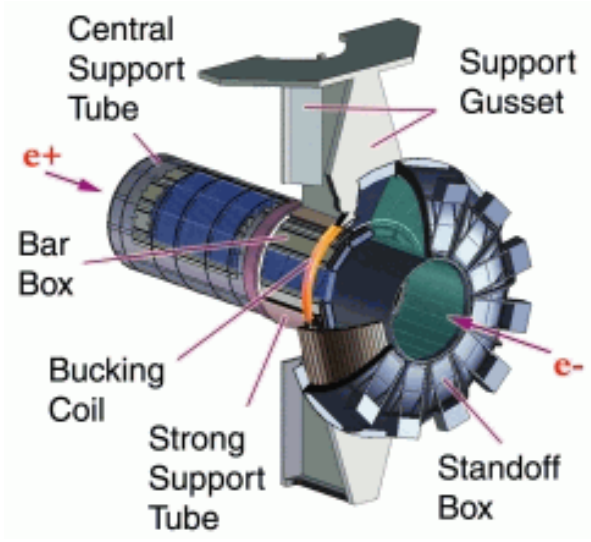

Figure 49. Exploded view of the DIRC mechanical support structure. The steel magnetic shield is not shown.

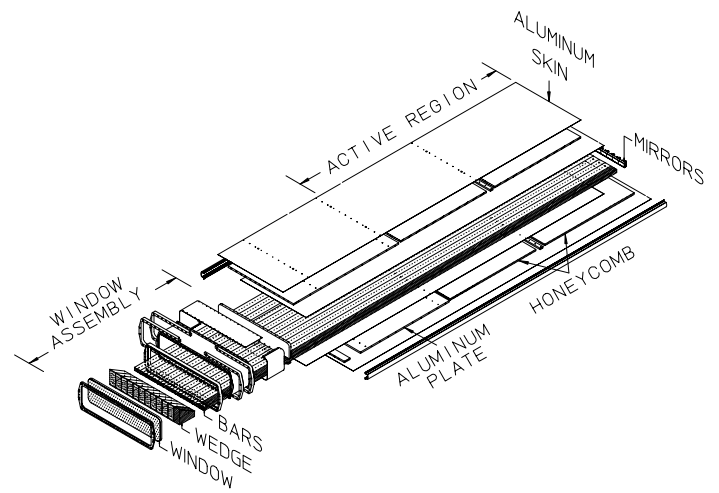

Figure 50. Schematics of the DIRC bar box assembly.

Tube (CST), attached to the SST via an aluminum transition flange. The CST is a thin, double-walled, cylindrical shell, using aircrafttype construction with stressed aluminum skins and bulkheads having riveted or glued joints. The CST also provides the support for the $\mathrm{DCH}$.

The standoff box is made of stainless steel, consisting of a cone, cylinder, and 12 sectors of PMTs. It contains about 6,000 liters of purified water. Water is used to fill this region because it is inexpensive and has an average index of refraction $(n \approx 1.346)$ reasonably close to that of

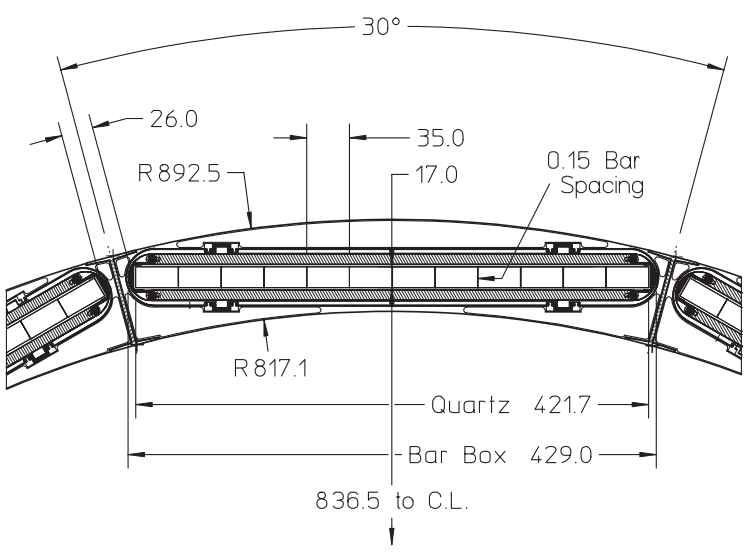

Figure 52. Transverse section of the nominal DIRC bar box imbedded in the CST. All dimensions are given in $\mathrm{mm}$.

fused silica, thus minimizing the total internal reflection at silica-water interface. Furthermore, its chromaticity index is a close match to that of fused silica, effectively eliminating dispersion at the silica-water interface. The steel gusset supports the standoff box. A steel shield, supplemented by a bucking coil, surrounds the standoff box to reduce the field in the PMT region to below 1 Gauss [28].

The PMTs at the rear of the standoff box lie on a surface that is approximately toroidal. Each of the 12 PMT sectors contains 896 PMTs (ETL model $9125[61,62])$ with 29 mm-diameter, in a closely packed array inside the water volume. A double o-ring water seal is made between the PMTs and the wall of the standoff box. The PMTs are installed from the inside of the standoff box and connected via a feed-through to a base mounted outside. The hexagonal light catcher cone is mounted in front of the photocathode of each PMT, which results in an effective active surface area light collection fraction of about $90 \%$. The geometry of the DIRC is shown in Figures 51 and 52 .

The DIRC occupies $80 \mathrm{~mm}$ of radial space in the central detector volume including supports and construction tolerances, with a total of about $17 \%$ radiation length thickness at normal incidence. The radiator bars subtend a solid angle corresponding to about $94 \%$ of the azimuth and 


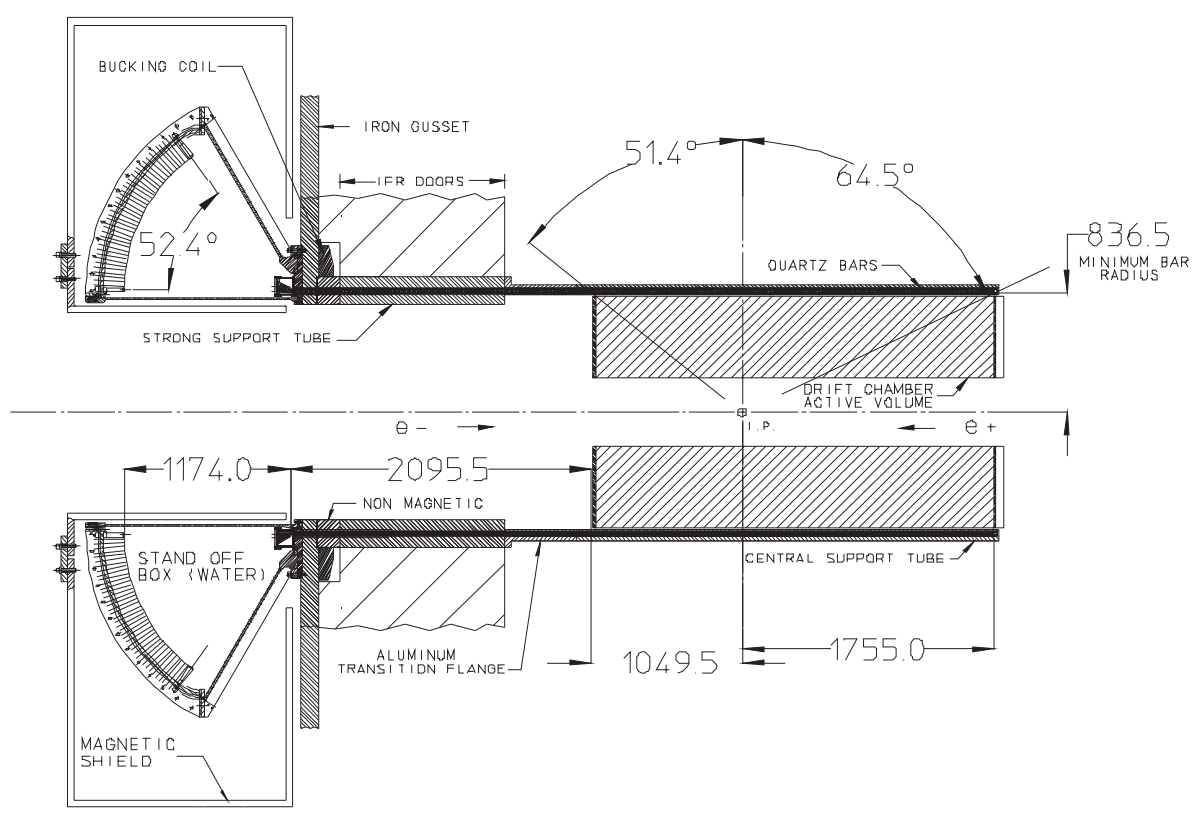

Figure 51. Elevation view of the nominal DIRC system geometry. For clarity, the end plug is not shown. All dimensions are given in $\mathrm{mm}$.

$83 \%$ of the c.m. polar angle cosine.

The distance from the end of the bar to the PMTs is $\sim 1.17 \mathrm{~m}$, which together with the size of the bars and PMTs, gives a geometric contribution to the single photon Cherenkov angle resolution of $\sim 7 \mathrm{mrad}$. This value is slightly larger than the rms spread of the photon production (dominated by a $\sim 5.4 \mathrm{mrad}$ chromatic term) and transmission dispersions. The overall single photon resolution is estimated to be about $10 \mathrm{mrad}$.

\subsubsection{Cherenkov Photon Detection Efficiency}

Figure 53 shows the contribution of various optical and electronic components of the DIRC to the Cherenkov photon detection efficiency as a function of wavelength. The data points pertain to a particle entering the center of the bar at $90^{\circ}$. A typical design goal for the photon transport in the bar was that no single component should contribute more than 10-20\% loss of detection efficiency. Satisfying this criterion required an extremely high internal reflection coefficient of the bar surfaces (greater than 0.9992 per bounce), so that about $80 \%$ of the light is main- tained after multiple bounces along the bars (365 bounces in the example of Figure 53). The ultraviolet cut-off is at $\sim 300 \mathrm{~nm}$, determined by the epoxy (Epotek 301-2 [63]) used to glue the fused silica bars together. The dominant contributor to the overall detection efficiency is the quantum efficiency of the PMT. Taking into account additional wavelength independent factors, such as the PMT packing fraction and the geometrical efficiency for trapping Cherenkov photons in the fused silica bars via total internal reflection, the expected number of photoelectrons $\left(N_{p e}\right)$ is $\sim 28$ for a $\beta=1$ particle entering normal to the surface at the center of a bar, and increases by over a factor of two in the forward and backward directions.

\subsubsection{DIRC Water System}

The DIRC water system is designed to maintain good transparency at wavelengths as small as $300 \mathrm{~nm}$. One way to achieve this is to use ultrapure, de-ionized water, close to the theoretical limit of $18 \mathrm{M} \Omega \mathrm{cm}$ resistivity. In addition, the water must be de-gassed and the entire system kept free of bacteria. Purified water is aggressive in at- 
O Water transmission $(1.2 \mathrm{~m})$

- Mirror reflectivity

- Internal reflection coeff. (365 bounces)

$\star$ Epotek 301-2 transmission $(25 \mu \mathrm{m})$

↔ EMI PMT 9215B quantum efficiency (Q.E.)

PMT Q.E. $\otimes$ PMT window transmission

$\triangle$ Final Cherenkov photon detection efficiency

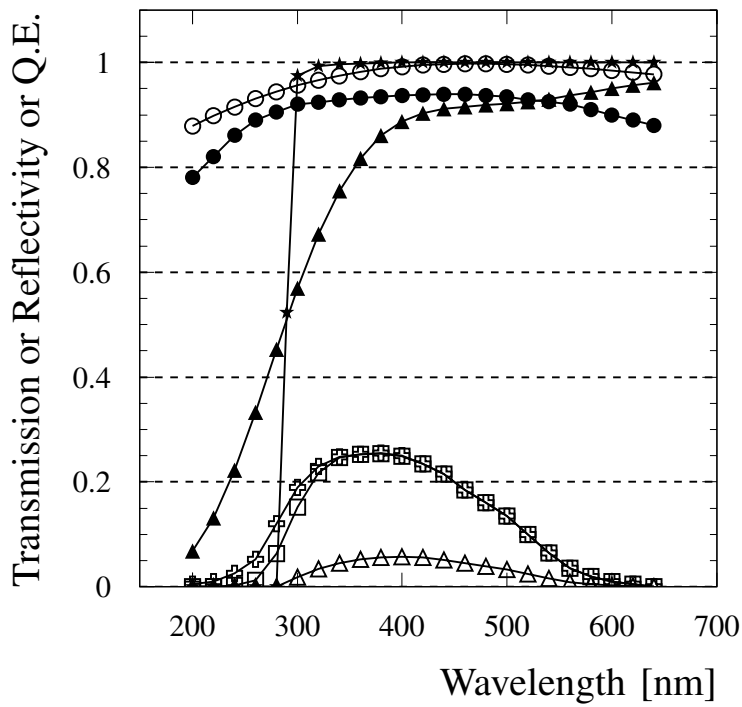

Figure 53. Transmission, reflectivity and quantum efficiency for various components of the DIRC as a function of wavelength for a $\beta=1$ particle at normal incidence to the center of a bar [64].

tacking many materials, and those in contact with the water were selected based on known compatability with purified water. To maintain the necessary level of water quality, most plumbing components are made of stainless steel or polyvinylidene fluoride.

The system contains an input line with six mechanical filters (three $10 \mu \mathrm{m}$, two $5 \mu \mathrm{m}$, and one $1 \mu \mathrm{m}$ ), a reverse osmosis unit, de-ionization beds, a Teflon microtube de-gasser and various pumps and valves. To prevent bacteria growth, it is equipped with a UV lamp (254 nm wavelength) and filters (two $1 \mu \mathrm{m}$, two $0.2 \mu \mathrm{m}$, and charcoal filters). Sampling ports are provided to check the water quality and to monitor resistivity,
$\mathrm{pH}$-value, temperature, and flow. A gravity feed return system prevents overpressure. The entire standoff box water volume can be recirculated up to four times a day.

The operating experience with the water system so far has been very good. The water volume is exchanged every ten hours and the resistivity of the water is typically $18 \mathrm{M} \Omega \mathrm{cm}$ in the supply line and $8-10 \mathrm{M} \Omega \mathrm{cm}$ in the return line at a temperature of about $23-26^{\circ} \mathrm{C}$. The $\mathrm{pH}$-value is about 6.5 and 6.6-6.7 in the supply and return water, respectively. The water transparency is routinely measured using lasers of three different wavelengths. The transmission is better than $92 \%$ per meter at $266 \mathrm{~nm}$ and exceeds $98 \%$ per meter at $325 \mathrm{~nm}$ and $442 \mathrm{~nm}$.

Potential leaks from the water seals between the bar boxes and the standoff box are detected by a water leak detection system of 20 custom water sensors in and about the bar box slots. Two commercial ultrasonic flow sensors are used to monitor water flow in two (normally dry) drain lines in addition to the 12 humidity sensors on a nitrogen gas output line from each bar box (see below). Should water be detected, a valve in a $100 \mathrm{~mm}$ diameter drain line is opened, and the entire system is drained in about 12 minutes.

All elements inside the standoff box (PMT, plastic PMT housing, gaskets, light catchers) were tested at normal and elevated temperatures to withstand the highly corrosive action of ultrapure water and to prevent its pollution. For instance, rhodium-plated mirrors on ULTEM support had to be used for the light catchers [59].

\subsubsection{DIRC Gas System}

Nitrogen gas from liquid nitrogen boil-off is used to prevent moisture from condensing on the bars, and used also to detect water leaks. The gas flows through each bar box at the rate of $100-200 \mathrm{~cm}^{3} / \mathrm{min}$, and is monitored for humidity to ensure that the water seal around the bar box remains tight. The gas is filtered through a molecular sieve and three mechanical filters to remove particulates $(7 \mu \mathrm{m}, 0.5 \mu \mathrm{m}$, and $0.01 \mu \mathrm{m})$. Dew points of the gas returned from the bar boxes are about $-40^{\circ} \mathrm{C}$. Approximately one third of the input nitrogen gas leaks from the bar boxes and 
keeps the bar box slots in the mechanical support structure free of condensation.

\subsection{Electronics \\ 8.4.1. DIRC PMT Electronics}

The DIRC PMT base contains a single printed circuit board, equipped with surface mounted components. The operating high voltage (HV) of the PMTs is $\sim 1.14 \mathrm{kV}$, with a range between 0.9 and $1.3 \mathrm{kV}$. Groups of 16 tubes are selected for uniformity of gain to allow their operation at a common HV provided from a single distribution board.

The HV is provided by a CAEN SY-527 high voltage distribution system. Each of the 12 sectors receives HV through 56 high voltage channels, distributed through a single cable bundle. Each voltage can be set between 0 and $1.6 \mathrm{kV}$.

\subsubsection{DIRC Front-End Electronics}

The DIRC front-end electronics (FEE) is designed to measure the arrival time of each Cherenkov photon detected by the PMT array $[65]$ to an accuracy that is limited by the intrinsic $1.5 \mathrm{~ns}$ transit time spread of the PMTs. The design contains a pipeline to deal with the L1 trigger latency of $12 \mu \mathrm{s}$, and can handle random background rates of up to $200 \mathrm{kHz} / \mathrm{PMT}$ with zero dead time. In addition, the pulse height spectra can be measured to ensure that the PMTs operate on the HV plateau. However, because the ADC information is not needed to reconstruct events, 64 PMTs are multiplexed onto a single $\mathrm{ADC}$ for monitoring and calibration.

The DIRC FEE is mounted on the outside of the standoff box and is highly integrated in order to minimize cable lengths and to retain the required single photoelectron sensitivity. Each of the 168 DIRC Front-end Boards (DFBs) processes 64 PMT inputs, containing eight custom analog chips along with their associated level translators, four custom-made TDC ICs, one 8bit flash ADC (FADC), two digitally controlled calibration signal generators, multi-event buffers, and test hardware.

The PMT signals are amplified, and pulseshaped by an eight-channel analog IC [66]. A digital pulse timed with the peak of the input pulse is output by a zero-crossing discriminator, as well as a pulse shaped by a CR-RC filter with 80 ns peaking time, which was chosen to allow for the ADC multiplexing. The multiplexer selects the channel to be digitized by the FADC for calibration.

The TDC IC [67] is a 16-channel TDC with $0.5 \mathrm{~ns}$ binning, input buffering, and selective readout of the data in time with the trigger. To cope with the L1 maximum trigger latency of $12 \mu \mathrm{s}$ and jitter of $1 \mu \mathrm{s}$, the selective readout process extracts data in time with the trigger within a programmable time window. The acceptance window width is programmable between 64 ns and $2 \mu \mathrm{s}$ and is typically set at $600 \mathrm{~ns}$. The twelve DIRC Crate Controllers (DCCs) that form the interface to the VME front-end crates are connected to six ROMs via $1.2 \mathrm{Gbit} / \mathrm{s}$ optical fibers.

\subsubsection{DAQ Feature Extraction}

Raw data from the DFBs are processed in the ROMs by a feature extraction algorithm before being transmitted to the segment and event builder. This software algorithm reduces the data volume by roughly $50 \%$ under typical background conditions. DFB data that contain errors are flagged and discarded. The only data errors seen to date have been traced to damaged DFBs that were replaced immediately. Because the dataflow system can reliably transmit at most $32 \mathrm{kBytes} /$ crate, the feature extraction must sometimes truncate data to limit the event size. Event data are replaced with a per-DFB occupancy summary when a ROM's hit occupancy exceeds $56 \%$, which occurs about once in $10^{4}$ events. An appropriate flag is inserted into the feature extraction output whenever truncation or deletion occurs. Errors, truncation, and feature extraction performance are continuously monitored online, and exceptions are either immediately corrected or logged for future action.

\subsubsection{DIRC Calibration}

The DIRC uses two independent approaches for a calibration of the unknown PMT time response and the delays introduced by the FEE and the fast control system. The first is a conventional pulser calibration. The second uses reconstructed 
tracks from collision data.

The pulser calibration is performed online using a light pulser system which generates precisely timed 1 ns duration light pulses from twelve, blue LEDs, one per sector. The LEDs are triggered by the global fast control calibration strobe command sent to the DCCs. The DCC triggers an individual LED for each sector upon receipt of calibration strobe. Pulses in adjacent sectors are staggered by $50 \mathrm{~ns}$ to prevent light crosstalk between sectors. The pulser is run at roughly $2 \mathrm{kHz}$ for the time delay calibration. The LED light is transmitted through approximately $47 \mathrm{~m}$-long optical fibers to diffusers mounted on the inner surface of the standoff box wall opposite the PMTs. This light produces about $10 \%$ photoelectron occupancy nearly uniformly throughout the standoff box.

Histograms of TDC times for each PMT are accumulated in parallel in the ROMs, and then fit to an asymmetric peak function. About 65,000 light pulses are used to determine the mean time delay of each of the PMTs in the standoff box to a statistical accuracy of better than 0.1 ns. The LED pulser is also used to monitor the phototube gains using the ADC readout. As with the TDC calibration, histograms and fits of the ADC spectrum are accumulated and fit in the ROM. A calibration run including both TDC and ADC information for all PMTs requires a few minutes, and is run once per day. Daily calibrations not only verify the time delays, but allow the detection of hardware failures.

The data stream calibration uses reconstructed tracks from the collision data. For calibration of the global time delay, the observed, uncalibrated times minus the expected arrival times, $\Delta t_{\gamma}$, are collected during the online prompt reconstruction processing. To calculate individual channel calibrations, $\Delta t_{\gamma}$ values for each DIRC channel are accumulated until statistics equivalent to 100,000 tracks are collected. The distribution for each channel is fit to extract the global time offset calibration.

The data stream and online pulser calibrations of the electronic delays, and of the PMT time response and gain yield fully consistent results, although the data stream results in $15 \%$ better timing resolution than the pulser calibration. The time delay values per channel are typically stable to an rms of less than 0.1 ns over more than one year of daily calibrations.

\subsubsection{DIRC Environmental Monitoring System}

The DIRC environmental monitoring system is divided into three parts, corresponding to three separate tasks. The first deals with the control and monitoring of the HV system for the PMTs. The second is devoted to monitoring low voltages related to the FEE. The third controls a variety of other detector parameter settings. An interlock system, based on a standard VME module (SIAM), is provided. For the purposes of the DIRC, three dedicated VME CPUs run the application code. The communication between the HV mainframes and the monitoring crate is achieved by a CAENET controller (V288). The HV monitor task controls the step sizes for ramping the $\mathrm{HV}$ up or down, as well as the communication of alarm conditions, and the values and limits for the HV and current of each channel.

The purpose of FEE monitoring is to control and monitor parameters related to the FEE. For each DIRC sector, a custom multipurpose board, the DCC, equipped with a microcontroller [68] incorporating the appropriate communication protocol (CANbus), is situated in the same crate as the DFB. All monitoring and control tasks are implemented on this card. The parameters monitored are the low voltages for the DFBs and DCCs, the status of the optical link (Finisar), the temperature on supply boards, and the VME crate status.

The third part of the monitoring system is based on a custom ADC VME board (VSAM), used to monitor various type of sensors: magnetic field sensors, an ensemble of 12 beam monitoring scalers, 16 CsI radiation monitors, the water level in the standoff box as well as its $\mathrm{pH}$-value, resistivity, and temperature.

\subsection{Operational Issues}

The DIRC was successfully commissioned and attained performance close to that expected from Monte Carlo simulation. The DIRC has been 

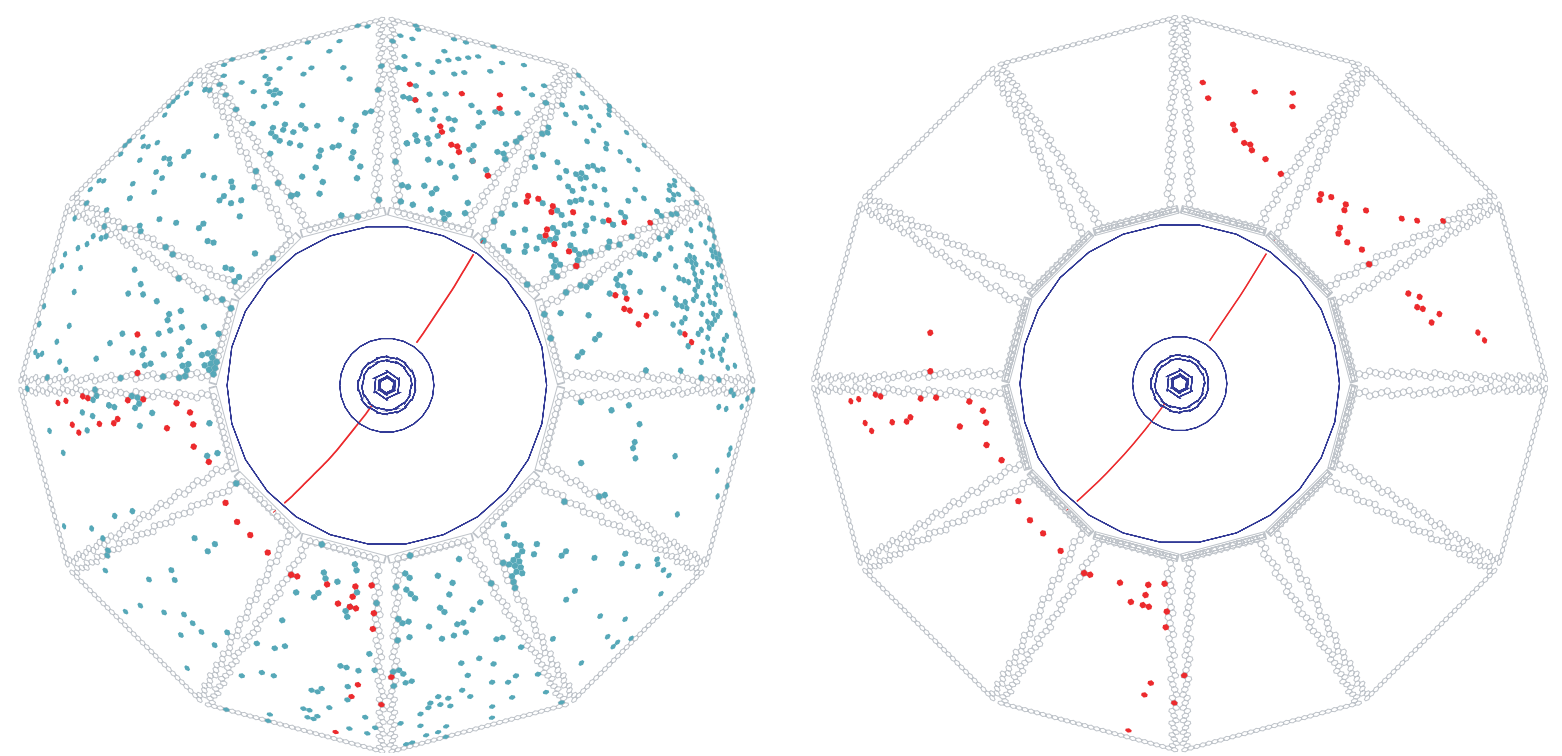

Figure 54. Display of an $e^{+} e^{-} \rightarrow \mu^{+} \mu^{-}$event reconstructed in BABAR with two different time cuts. On the left, all DIRC PMTs with signals within the \pm 300 ns trigger window are shown. On the right, only those PMTs with signals within 8 ns of the expected Cherenkov photon arrival time are displayed.

robust and stable, and, indeed, serves also as a background detector for PEP-II tuning. Figure 54 shows a typical di-muon event $\left(e^{+} e^{-} \rightarrow\right.$ $\left.\mu^{+} \mu^{-}\right)$. In addition to the signals caused by the Cherenkov light from the two tracks, about 500 background signals can be seen in the readout window of $\pm 300 \mathrm{~ns}$. This background is dominated by low energy photons from the PEP-II machine hitting the standoff box. Some care in machine tuning is required to stay under a noise limit of about $200 \mathrm{kHz} /$ tube imposed by limited DAQ throughput. Lead shielding has been installed around the beam line components just outside the backward endcap, and has substantially reduced this background.

After about two years of running, approximately $99.7 \%$ of PMTs and electronic channels are operating with nominal performance.

Some deterioration of the PMT front glass windows (made of B53 Borosilicate glass) that are immersed in the ultra-pure water of the standoff box has been observed. For most of the tubes, the observable effect is typically a slight cloudiness, but for about 50 tubes, it is much more pronounced. Extensive R\&D has demonstrated that the effect is associated with a loss of sodium and boron from the surface of the glass [69]. For most tubes, the leaching rate is a few microns per year, and is expected to be acceptable for the full projected ten year lifetime of the experiment. However, for the $\sim 50$ tubes, the incorrect glass was used by the manufacturer. That glass does not contain zinc, making it much more susceptible to rapid leaching. This leaching may eventually lead to either a loss of performance, or some risk of mechanical failure of the face plates for these tubes. Direct measurements of the number of Cherenkov photons observed in di-muon events as a function of time suggest that the total loss of photons from all sources is less than $2 \%$ /year.

\subsection{Data Analysis and Performance}

Figure 54 shows the pattern of Cherenkov photons in a di-muon event, before and after reconstruction. The time distribution of real Cherenkov photons from a single event is of order $\sim 50$ ns wide, and during normal data-taking they are accompanied by hundreds of random photons in a flat background within the trigger acceptance window. Given a track pointing at a particular 
fused silica bar and a candidate signal in a PMT within the optical phase space of that bar, the Cherenkov angle is determined up to a 16 -fold ambiguity: top or bottom, left or right, forward or backward, and wedge or no-wedge reflections. The goal of the reconstruction program is to associate the correct track with the candidate PMT signal, with the requirement that the transit time of the photon from its creation in the bar to its detection at the PMT be consistent with the measurement error of $\sim 1.5 \mathrm{~ns}$.

\subsubsection{Reconstruction}

An unbinned maximum likelihood formalism is used to incorporate all information provided by the space and time measurements from the DIRC.

The emission angle and the arrival time of the Cherenkov photons are reconstructed from the observed space-time coordinates of the PMT signals, transformed into the Cherenkov coordinate system $\left(\theta_{c}, \phi_{c}\right.$, and $\left.\delta t\right)$ as follows: The known spatial position of the bar through which the track passed and the PMTs whose signal times lie within the readout window of $\pm 300 \mathrm{~ns}$ from the trigger are used to calculate the threedimensional vector pointing from the center of the bar end to the center of each tube. This vector is then extrapolated into the radiator bar (using Snell's law). This procedure defines, up to the 16-fold ambiguity described above, the angles $\theta_{c}$ and $\phi_{c}$ of a photon.

The DIRC time measurement represents the third dimension of the photomultiplier hit reconstruction. The timing resolution is not competitive with the position information for Cherenkov angle reconstruction, but timing information is used to suppress background hits from the beam induced background and, more importantly, exclude other tracks in the same event as the source of the photon. Timing information is also used to resolve the forward-backward and wedge ambiguities in the hit-to-track association.

The relevant observable to distinguish between signal and background photons is the difference between the measured and expected photon arrival time, $\Delta t_{\gamma}$. It is calculated for each photon using the track time-of-flight (assuming it to be a charged pion), the measured time of the can- didate signal in the PMT and the photon propagation time within the bar and the water filled standoff box. The time information and the requirement of using only physically possible photon propagation paths reduce the number of ambiguities from 16 to typically 3 . Applying the time information also substantially improves the correct matching of photons with tracks and reduces the number of accelerator induced background signals by approximately a factor 40 , as illustrated in Figure 54.

The reconstruction routine currently provides a likelihood value for each of the five stable particle types $(\mathrm{e}, \mu, \pi, \mathrm{K}, \mathrm{p})$ if the track passes through the active volume of the DIRC. These likelihoods are calculated in an iterative process by maximizing the likelihood value for the entire event while testing different hypotheses for each track. If enough photons are found, a fit of $\theta_{c}$ and the number of observed signal and background photons are calculated for each track.

\subsubsection{Results}

The parameters of expected DIRC performance were derived from extensive studies with a variety of prototypes, culminating with a full-size prototype in a beam at CERN [70]. The test beam results were well-described by Monte Carlo simulations of the detector. The performance of the full detector is close to expectations, and additional offline work, particularly on geometrical alignment, is expected to lead to further improvements.

In the absence of correlated systematic errors, the resolution $\left(\sigma_{C, \text { track }}\right)$ on the track Cherenkov angle should scale as

$\sigma_{C, \text { track }}=\frac{\sigma_{C, \gamma}}{\sqrt{N_{p e}}}$,

where $\sigma_{C, \gamma}$ is the single photon Cherenkov angle resolution, and $N_{p e}$ is the number of photons detected. Figure 55(a) shows the single photon angular resolution obtained from di-muon events. There is a broad background of less than $10 \%$ relative height under the peak, that originates mostly from track-associated sources, such as $\delta$ rays, and combinatorial background. The width of the peak translates to a resolution of about 

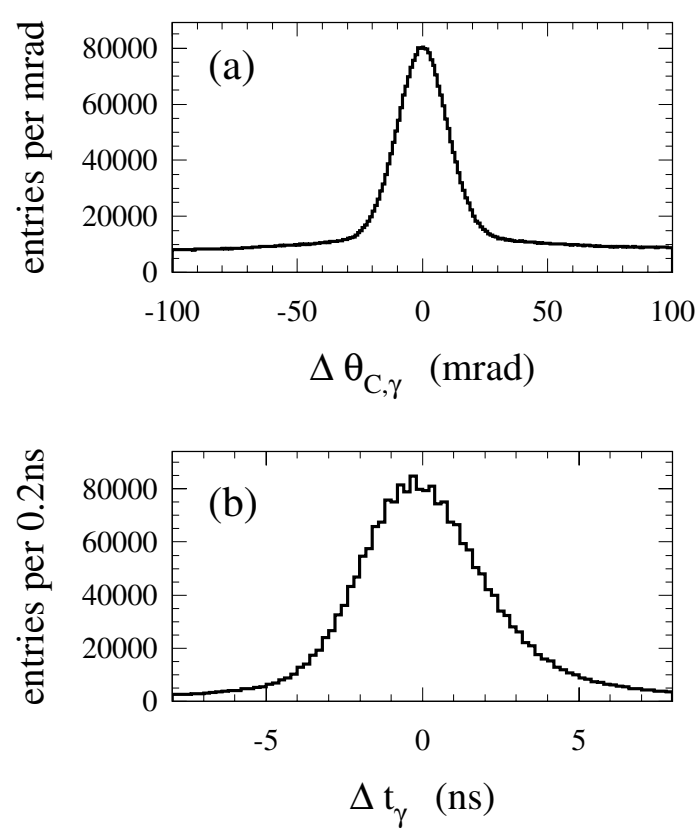

Figure 55. The difference between (a) the measured and expected Cherenkov angle for single photons, $\Delta \theta_{c, \gamma}$, and (b) the measured and expected photon arrival time, for single muons in $\mu^{+} \mu^{-}$events.

$10.2 \mathrm{mrad}$, in good agreement with the expected value. The measured time resolution (see Figure $55(\mathrm{~b}))$ is $1.7 \mathrm{~ns}$, close to the intrinsic $1.5 \mathrm{~ns}$ transit time spread of the PMTs.

The number of photoelectrons shown in Figure 56 varies between 20 for small polar angles at the center of the barrel and 65 at large polar angles. This variation is well reproduced by Monte Carlo simulation, and can be understood from the geometry of the DIRC. The number of Cherenkov photons varies with the pathlength of the track in the radiator, it is smallest at perpendicular incidence at the center and increases towards the ends of the bars. In addition, the fraction of photons trapped by total internal reflection rises with larger values of $\left|\cos \theta_{\text {track }}\right|$. This increase in the number of photons for forward going tracks is a good match to the increase in momentum and thus benefits the DIRC performance.

With the present alignment, the track Cherenkov angle resolution for di-muon events is

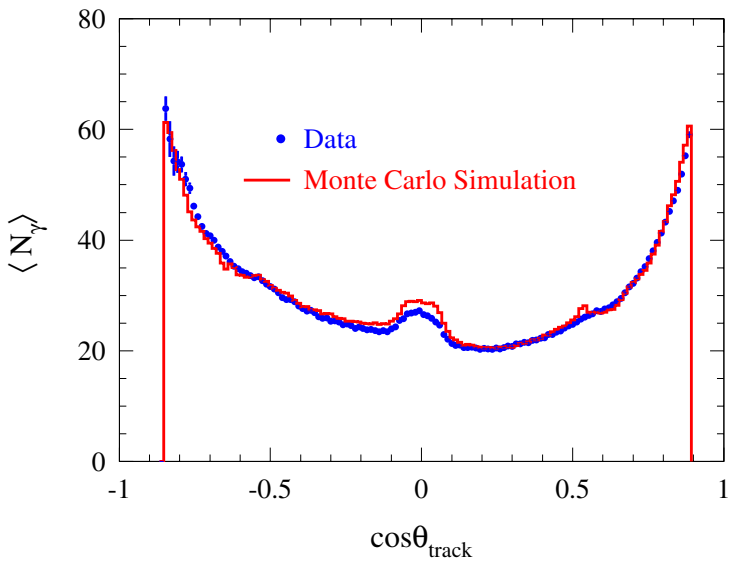

Figure 56. Number of detected photons versus track polar angle for reconstructed tracks in dimuon events compared to Monte Carlos simulation. The mean number of photons in the simulation has been tuned to match the data.

shown in Figure 57. The width of the fitted Gaussian distribution is $2.5 \mathrm{mrad}$ compared to the design goal of $2.2 \mathrm{mrad}$. From the measured single track resolution versus momentum in di-muon events and the difference between the expected Cherenkov angles of charged pions and kaons, the pion-kaon separation power of the DIRC can be inferred. As shown in Figure 58, the expected separation between kaons and pions at $3 \mathrm{GeV} / c$ is about $4.2 \sigma$, within $15 \%$ of the design goal.

Figure 59 shows an example of the use of the DIRC for particle identification. The $K \pi$ invariant mass spectra are shown with and without the use of the DIRC for kaon identification. The peak corresponds to the decay of the $D^{0}$ particle.

The efficiency for correctly identifying a charged kaon that traverses a radiator bar and the probability to wrongly identify a pion as a kaon are determined using $D^{0} \rightarrow K^{-} \pi^{+}$decays selected kinematically from inclusive $D^{*}$ production and are shown as a function of the track momentum in Figure 60 for a particular choice of particle selection criteria. The mean kaon selection efficiency and pion misidentification are $96.2 \pm 0.2 \%$ (stat.) and $2.1 \pm 0.1 \%$ (stat.), respectively. 


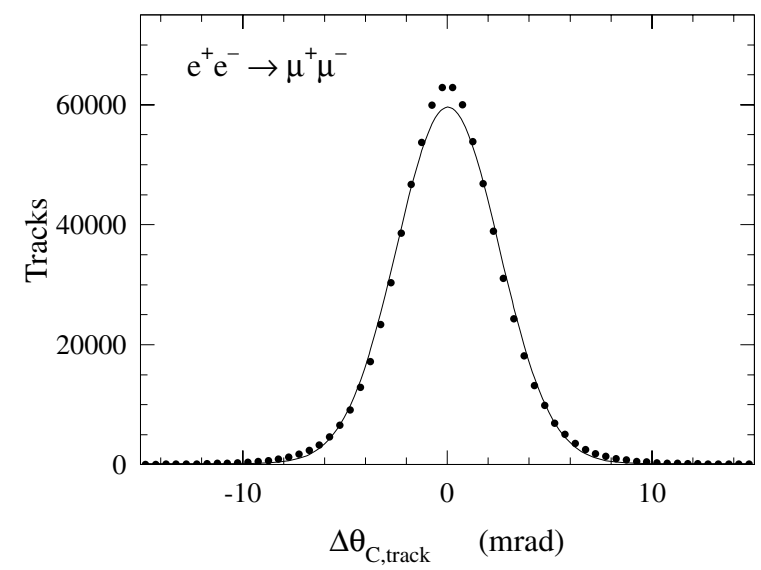

Figure 57. The difference between the measured and expected Cherenkov angle, $\Delta \theta_{c, \text { track }}$, for single muons in $\mu^{+} \mu^{-}$events. The curve represents a Gaussian distribution fit to the data with a width of $2.5 \mathrm{mrad}$.

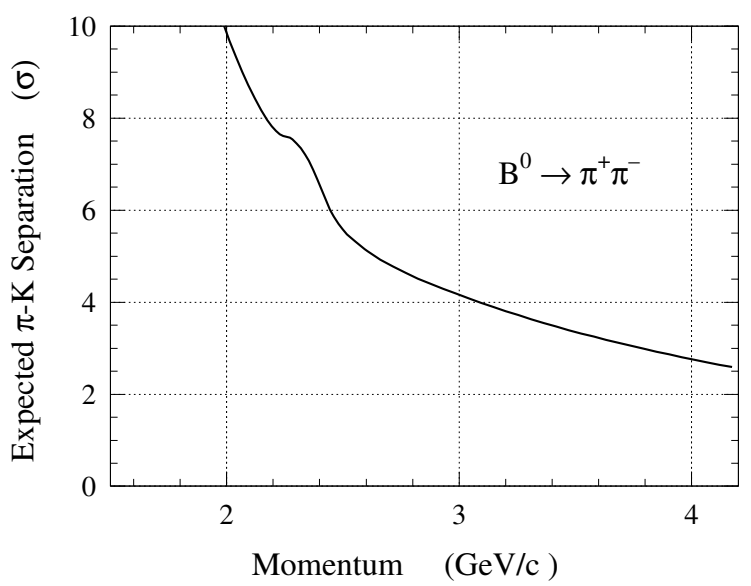

Figure 58. Expected $\pi$-K separation in $B^{0} \rightarrow$ $\pi^{+} \pi^{-}$events versus track momentum inferred from the measured Cherenkov angle resolution and number of Cherenkov photons per track in di-muon events.

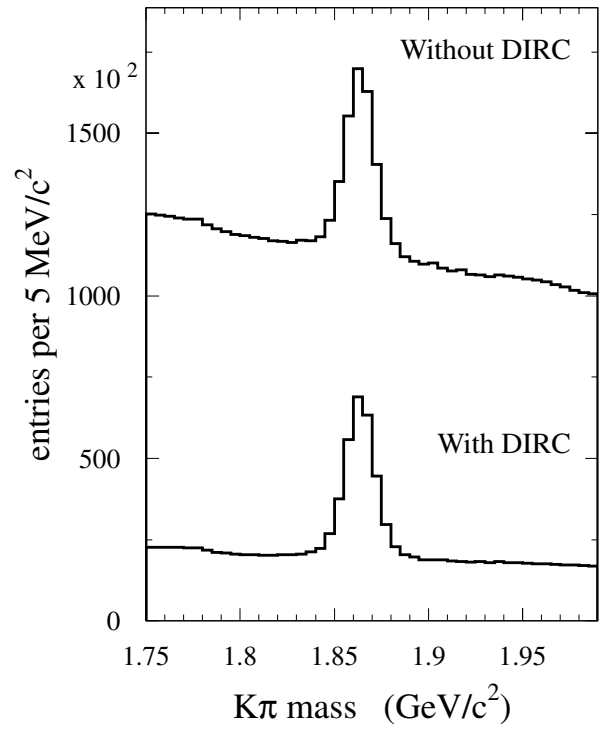

Figure 59. Invariant $\mathrm{K} \pi$ inclusive mass spectrum with and without the use of the DIRC for kaon identification. The mass peak corresponds to the decay of the $D^{0}$ particle.

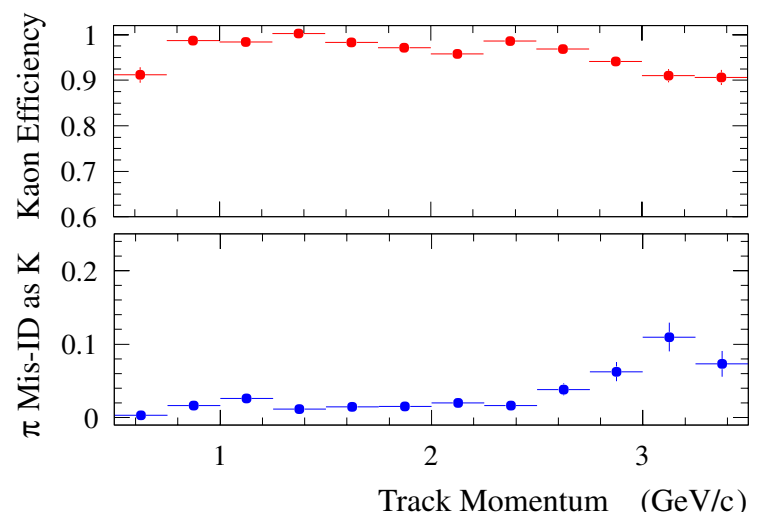

Figure 60. Efficiency and misidentification probability for the selection of charged kaons as a function of track momentum, determined using $D^{0} \rightarrow K^{-} \pi^{+}$decays selected kinematically from inclusive $D^{*}$ production. 


\subsection{Summary}

The DIRC is a novel ring-imaging Cherenkov detector that is well-matched to the hadronic PID requirements of BABAR. The DIRC has been robust and stable and, two years after installation, about $99.7 \%$ of all PMTs and electronic channels are operating with nominal performance. Additional shielding in the standoff box tunnel region should reduce the sensitivity to beam-induced backgrounds, as should faster FEE, both installed during the winter 2000-2001 shutdown. At luminosities around $1 \times 10^{34} \mathrm{~cm}^{-2} \mathrm{~s}^{-1}$, the TDC IC will have to be replaced with a faster version and deeper buffering. The design process for this is underway.

The detector performance achieved is rather close to that predicted by the Monte Carlo simulations. Alignment and further code developments are expected to further improve performance.

\section{Electromagnetic Calorimeter}

\subsection{Purpose and Design}

The electromagnetic calorimeter (EMC) is designed to measure electromagnetic showers with excellent efficiency, and energy and angular resolution over the energy range from $20 \mathrm{MeV}$ to $9 \mathrm{GeV}$. This capability allows the detection of photons from $\pi^{0}$ and $\eta$ decays as well as from electromagnetic and radiative processes. By identifying electrons, the EMC contributes to the flavor tagging of neutral $B$ mesons via semi-leptonic decays, to the reconstruction of vector mesons like $J / \psi$, and to the study of semi-leptonic and rare decays of $B$ and $D$ mesons, and $\tau$ leptons. The upper bound of the energy range is set by the need to measure QED processes, like $e^{+} e^{-} \rightarrow e^{+} e^{-}(\gamma)$ and $e^{+} e^{-} \rightarrow \gamma \gamma$, for calibration and luminosity determination. The lower bound is set by the need for highly efficient reconstruction of $B$ meson decays containing multiple $\pi^{0} \mathrm{~s}$ and $\eta^{0} \mathrm{~s}$.

\subsubsection{Requirements}

The measurement of extremely rare decays of $B$ mesons containing $\pi^{0} \mathrm{~s}\left(\right.$ e.g., $\left.B^{0} \rightarrow \pi^{0} \pi^{0}\right)$ poses the most stringent requirements on energy resolution, namely of order $1-2 \%$. Below energies of
$2 \mathrm{GeV}$, the $\pi^{0}$ mass resolution is dominated by the energy resolution. At higher energies, the angular resolution becomes dominant, and therefore it is required to be of the order of a few mrad.

Furthermore, the EMC has to be compatible with the $1.5 \mathrm{~T}$ field of the solenoid and operate reliably over the anticipated ten-year lifetime of the experiment. To achieve excellent resolution, stable operating conditions have to be maintained. Temperatures and the radiation exposure must be closely monitored, and precise calibrations of the electronics and energy response over the full dynamic range must be performed frequently.

\subsubsection{Design Considerations}

The requirements stated above lead to the choice of a hermetic, total-absorption calorimeter, composed of a finely segmented array of thallium-doped cesium iodide $(\mathrm{CsI}(\mathrm{Tl})$ ) crystals. The crystals are read out with silicon photodiodes that are matched to the spectrum of scintillation light. Recent experience at CLEO [71] has demonstrated the suitability of this choice for physics at the $\Upsilon(4 S)$ resonance.

The energy resolution of a homogeneous crystal calorimeter can be described empirically in terms of a sum of two terms added in quadrature

$\frac{\sigma_{E}}{E}=\frac{a}{4 \sqrt{E(\mathrm{GeV})}} \oplus b$,

where $E$ and $\sigma_{E}$ refer to the energy of a photon and its rms error, measured in GeV. The energy dependent term $a$ arises primarily from the fluctuations in photon statistics, but it is also impacted by electronic noise of the photon detector and electronics. Furthermore, beam-generated background will lead to large numbers of additional photons that add to the noise. This term is dominant at low energies. The constant term, $b$, is dominant at higher energies $(>1 \mathrm{GeV})$. It arises from non-uniformity in light collection, leakage or absorption in the material between and in front of the crystals, and uncertainties in the calibrations. Most of these effects can be influenced by design choices, and they are stable with time. Others will be impacted by changes in the operating conditions, like variations in temperature, electronics gain, and noise, as well as by radiation damage 
caused by beam-generated radiation.

The angular resolution is determined by the transverse crystal size and the distance from the interaction point. It can also be empirically parameterized as a sum of an energy dependent and a constant term,

$\sigma_{\theta}=\sigma_{\phi}=\frac{c}{\sqrt{E(\mathrm{GeV})}}+d$,

where the energy $E$ is measured in $\mathrm{GeV}$. The design of the EMC required a careful optimization of a wide range of choices, including the crystal material and dimensions, the choice of the photon detector and readout electronics, and the design of a calibration and monitoring system. These choices were made on the basis of extensive studies, prototyping and beam tests [72], and Monte Carlo simulation, taking into account limitations of space and the impact of other BABAR detector systems.

Under ideal conditions, values for the energy resolution parameters $a$ and $b$ close to $1-2 \%$ could be obtained. A position resolution of a few $\mathrm{mm}$ will translate into an angular resolution of a few mrad; corresponding parameter values are $c \approx$ $3 \mathrm{mrad}$ and $d \approx 1 \mathrm{mrad}$.

However in practice, such performance is very difficult to achieve in a large system with a small, but unavoidable amount of inert material and gaps, limitations of electronics, and background in multi-particle events, plus contributions from beam-generated background.

Though in $\mathrm{CsI}(\mathrm{Tl})$ the intrinsic efficiency for the detection of photons is close to $100 \%$ down to a few $\mathrm{MeV}$, the minimum measurable energy in colliding beam data is expected to be about $20 \mathrm{MeV}$, a limit that is largely determined by beam- and event-related background and the amount of material in front of the calorimeter. Because of the sensitivity of the $\pi^{0}$ efficiency to the minimum detectable photon energy, it is extremely important to keep the amount of material in front of the EMC to the lowest possible level.

\subsubsection{CsI(Tl) Crystals}

Thallium-doped CsI meets the needs of BABAR in several ways. Its properties are listed in Table 11. The high light yield and small Molière
Table 11

Properties of $\mathrm{CsI}(\mathrm{Tl})$.

\begin{tabular}{ll}
\hline Parameter & Values \\
\hline Radiation Length & $1.85 \mathrm{~cm}$ \\
Molière Radius & $3.8 \mathrm{~cm}$ \\
Density & $4.53 \mathrm{~g} / \mathrm{cm}^{3}$ \\
Light Yield & $50,000 \mathrm{\gamma} / \mathrm{MeV}$ \\
Light Yield Temp. Coeff. & $0.28 \% /{ }^{\circ} \mathrm{C}$ \\
Peak Emission $\lambda_{\max }$ & $565 \mathrm{~nm}$ \\
Refractive Index $\left(\lambda_{\max }\right)$ & 1.80 \\
Signal Decay Time & $680 \mathrm{~ns}(64 \%)$ \\
& $3.34 \mu \mathrm{s}(36 \%)$ \\
\hline
\end{tabular}

radius allow for excellent energy and angular resolution, while the short radiation length allows for shower containment at $B A B A R$ energies with a relatively compact design. Furthermore, the high light yield and the emission spectrum permit efficient use of silicon photodiodes which operate well in high magnetic fields. The transverse size of the crystals is chosen to be comparable to the Molière radius achieving the required angular resolution at low energies while appropriately limiting the total number of crystals (and readout channels).

\subsection{Layout and Assembly}

\subsubsection{Overall Layout}

The EMC consists of a cylindrical barrel and a conical forward endcap. It has full coverage in azimuth and extends in polar angle from $15.8^{\circ}$ to $141.8^{\circ}$ corresponding to a solid-angle coverage of $90 \%$ in the c.m. system (see Figure 61 and Table 12). The barrel contains 5,760 crystals arranged in 48 distinct rings with 120 identical crystals each. The endcap holds 820 crystals arranged in eight rings, adding up to a total of 6,580 crystals. The crystals have a tapered trapezoidal cross section. The length of the crystals increases from $29.6 \mathrm{~cm}$ in the backward to $32.4 \mathrm{~cm}$ in the forward direction to limit the effects of shower leakage from increasingly higher energy particles.

To minimize the probability of pre-showering, the crystals are supported at the outer radius, with only a thin gas seal at the front. The barrel and outer five rings of the endcap have less than $0.3-0.6 X_{0}$ of material in front of the crystal faces. 


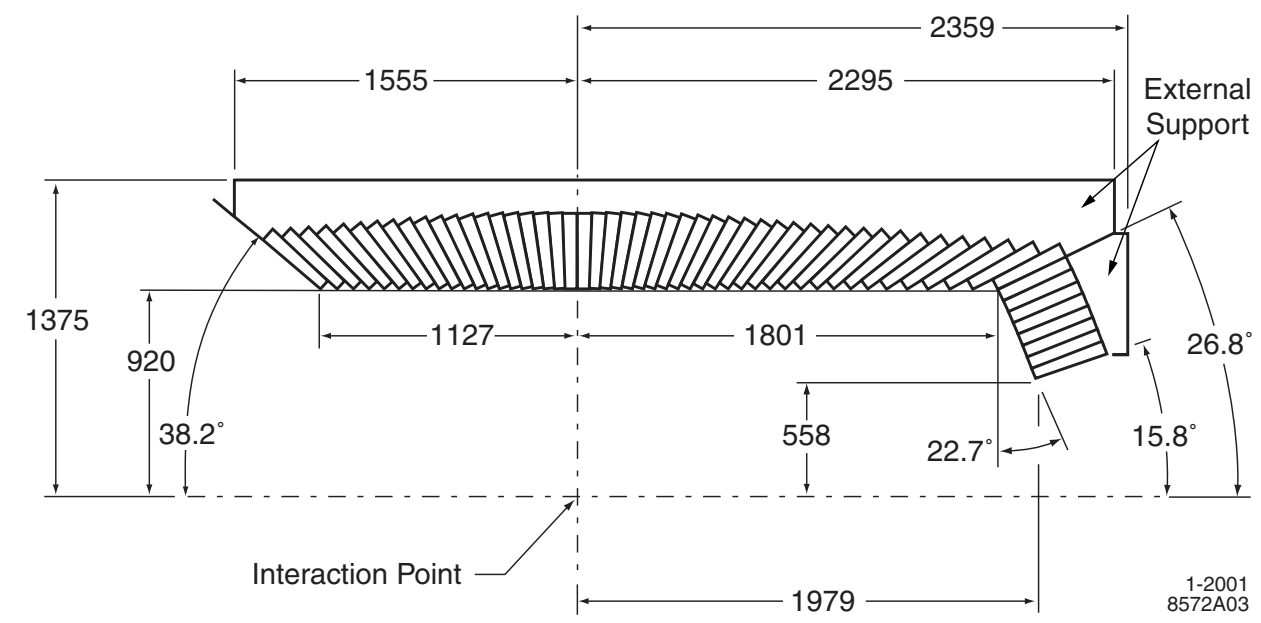

Figure 61. A longitudinal cross section of the EMC (only the top half is shown) indicating the arrangement of the 56 crystal rings. The detector is axially symmetric around the $z$-axis. All dimensions are given in $\mathrm{mm}$.

Table 12

Layout of the EMC, composed of 56 axially symmetric rings, each consisting of CsI crystals of identical dimensions.

\begin{tabular}{cccc}
\hline $\begin{array}{c}\theta \text { Interval } \\
\text { (radians) }\end{array}$ & $\begin{array}{c}\text { Length } \\
\left(X_{0}\right)\end{array}$ & $\begin{array}{c}\# \\
\text { Rings }\end{array}$ & $\begin{array}{c}\text { Crystals } \\
\text { /Ring }\end{array}$ \\
\hline \multicolumn{4}{c}{ Barrel } \\
\hline $2.456-1.214$ & 16.0 & 27 & 120 \\
$1.213-0.902$ & 16.5 & 7 & 120 \\
$0.901-0.655$ & 17.0 & 7 & 120 \\
$0.654-0.473$ & 17.5 & 7 & 120 \\
\hline \multicolumn{5}{c}{ Endcap } \\
\hline $0.469-0.398$ & 17.5 & 3 & 120 \\
$0.397-0.327$ & 17.5 & 3 & 100 \\
$0.326-0.301$ & 17.5 & 1 & 80 \\
$0.300-0.277$ & 16.5 & 1 & 80 \\
\hline
\end{tabular}

The SVT support structure and electronics, as well as the B1 dipole shadow the inner three rings of the endcap, resulting in up to $3.0 X_{0}$ for the innermost ring. The principal purpose of the two innermost rings is to enhance shower containment for particles close to the acceptance limit.

\subsubsection{Crystal Fabrication and Assembly}

The crystals were grown in boules from a melt of CsI salt doped with $0.1 \%$ thallium [73]. They were cut from the boules, machined into tapered trapezoids (Figure 62) to a tolerance of $\pm 150 \mu \mathrm{m}$, and then polished [74]. The transverse dimensions of the crystals for each of the 56 rings vary to achieve the required hermetic coverage. The typical area of the front face is $4.7 \times 4.7 \mathrm{~cm}^{2}$, while the back face area is typically $6.1 \times 6.0 \mathrm{~cm}^{2}$. The crystals act not only as a total-absorption scintillating medium, but also as a light guide to collect light at the photodiodes that are mounted on the rear surface. At the polished crystal surface light is internally reflected, and a small fraction is transmitted. The transmitted light is recovered in part by wrapping the crystal with two layers of diffuse white reflector [75,76], each $165 \mu \mathrm{m}$ thick. The uniformity of light yield along the wrapped crystal was measured by recording the signal from a highly collimated radioactive source at 20 points along the length of the crystal. The light yield was required to be uniform to within $\pm 2 \%$ in the front half of the crystal; the limit increased linearly up to a maximum of $\pm 5 \%$ at the rear face. Adjustments were made on individual crystals to meet these criteria by selectively roughing or pol- 


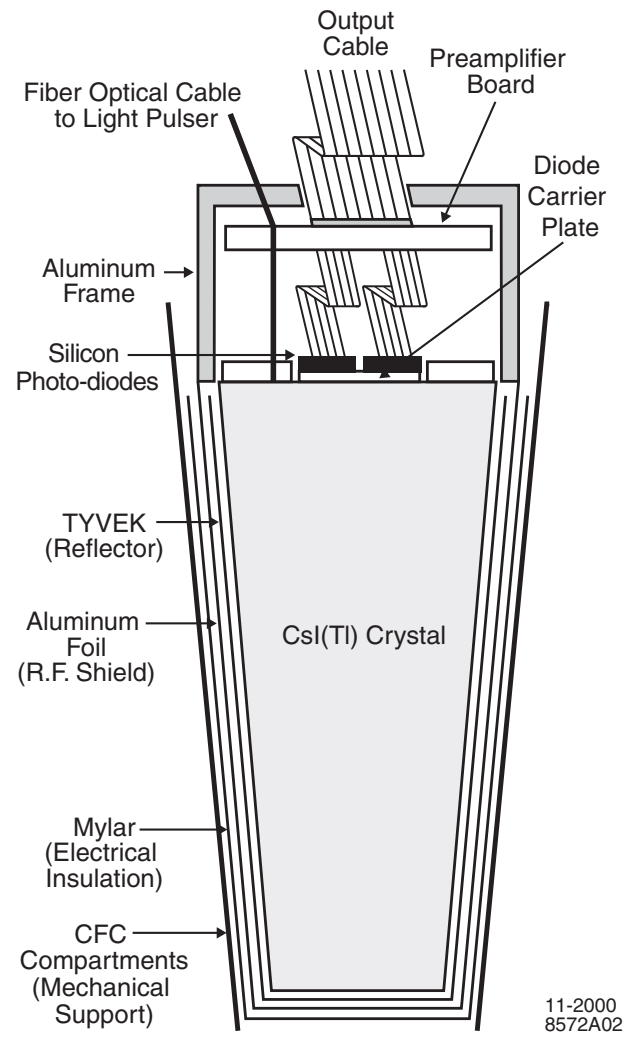

Figure 62. A schematic of the wrapped $\mathrm{CsI}(\mathrm{Tl})$ crystal and the front-end readout package mounted on the rear face. Also indicated is the tapered, trapezoidal CFC compartment, which is open at the front. This drawing is not to scale.

ishing the crystal surface to reduce or increase its reflectivity.

Following these checks, the crystals were further wrapped in $25 \mu \mathrm{m}$ thick aluminum foil which was electrically connected to the metal housing of the photodiode-preamplifier assembly to provide a Faraday shield. The crystals were covered on the outside with a $13 \mu \mathrm{m}$-thick layer of mylar to assure electrical isolation from the external support.

\subsubsection{Photodiodes and Preamplifier Assembly}

The photon detector consists of two $2 \times 1 \mathrm{~cm}^{2}$ silicon PIN diodes glued to a transparent $1.2 \mathrm{~mm}$ - thick polysterene substrate that, in turn, is glued to the center of the rear face of the crystal by an optical epoxy [77] to maximize light transmission [78]. The surrounding area of the crystal face is covered by a plastic plate coated with white reflective paint [79]. The plate has two $3 \mathrm{~mm}-$ diameter penetrations for the fibers of the light pulser monitoring system.

As part of the quality control process, the $1.836 \mathrm{MeV}$ photon line from ${ }^{88} \mathrm{Y}$ radioactive source was used to measure the light yield of every crystal-diode assembly, employing a preamplifier with $2 \mu$ s Gaussian shaping. The resulting signal distribution had a mean and rms width of 7300 and 890 photoelectrons/MeV, respectively; none of the crystals had a signal of less than 4600 photoelectrons/MeV $[78,80]$.

Each of the diodes is directly connected to a low-noise preamplifier. The entire assembly is enclosed by an aluminum fixture as shown in Figure 62 . This fixture is electrically coupled to the aluminum foil wrapped around the crystal and thermally coupled to the support frame to dissipate the heat load from the preamplifiers.

Extensive aging tests were performed to ascertain that the diodes and the preamplifiers met the ten-year lifetime requirements. In addition, daily thermal cycles of $\pm 5^{\circ} \mathrm{C}$ were run for many months to assure that the diode-crystal epoxy joint could sustain modest temperature variations.

\subsubsection{Crystal Support Structure}

The crystals are inserted into modules that are supported individually from an external support structure. This structure is built in three sections, a cylinder for the barrel and two semicircular structures for the forward endcap. The barrel support cylinder carries the load of the barrel modules plus the forward endcap to the magnet iron through four flexible supports. These supports decouple and dampen any acceleration induced by movements of the magnet iron during a potential earthquake.

The modules are built from tapered, trapezoidal compartments made from carbon-fiberepoxy composite (CFC) with $300 \mu$ m-thick walls (Figure 63). Each compartment loosely holds a single wrapped and instrumented crystal and 


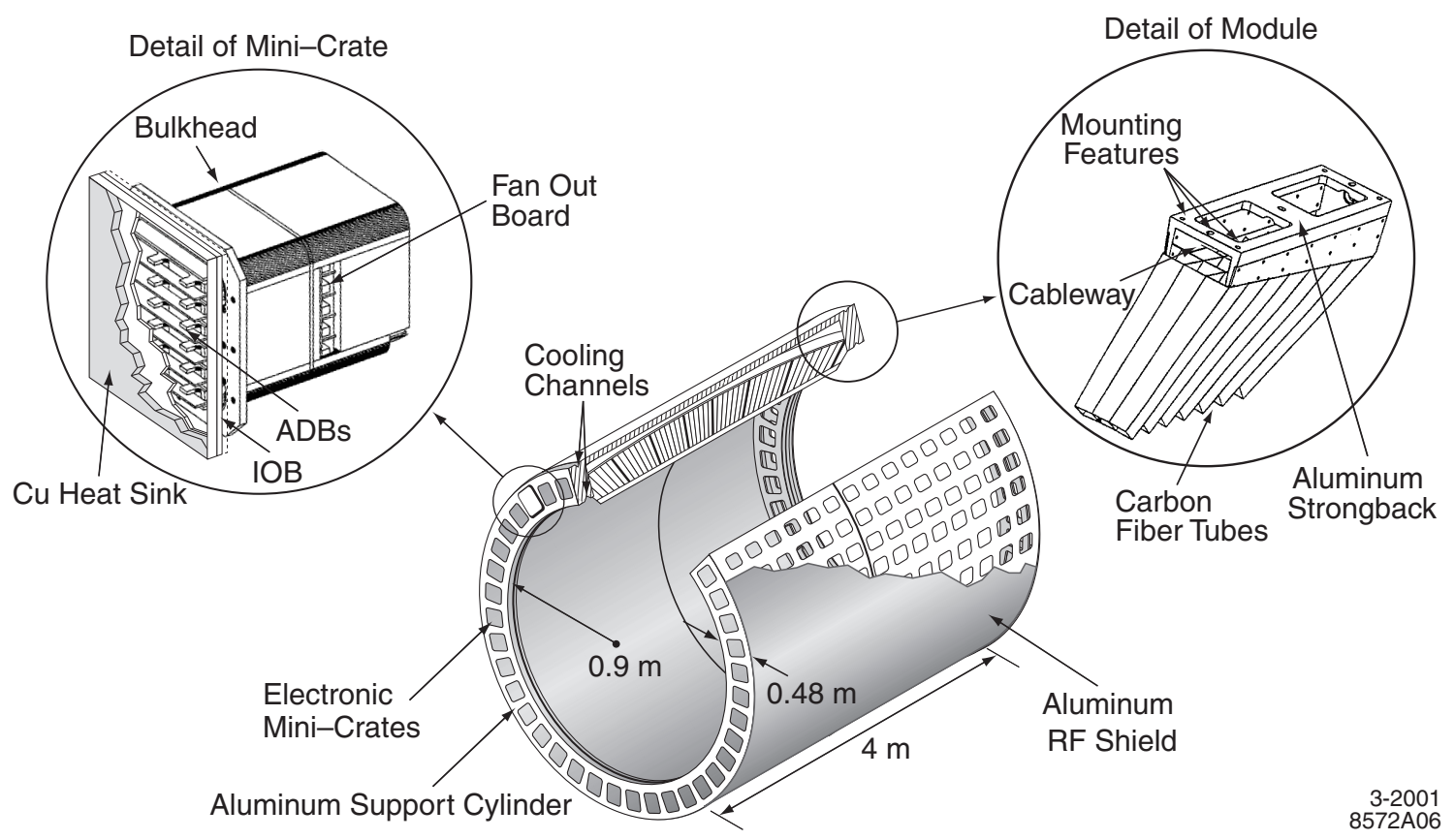

Figure 63. The EMC barrel support structure, with details on the modules and electronics crates (not to scale).

thus assures that the forces on the crystal surfaces never exceed its own weight. Each module is surrounded by an additional layer of $300 \mu \mathrm{m}$ CFC to provide additional strength. The modules are bonded to an aluminum strong-back that is mounted on the external support. This scheme minimizes inter-crystal materials while exerting minimal force on the crystal surfaces; this prevents deformations and surface degradation that could compromise performance. By supporting the modules at the back, the material in front of the crystals is kept to a minimum.

The barrel section is divided into 280 separate modules, each holding 21 crystals $(7 \times 3$ in $\theta \times \phi)$. After the insertion of the crystals, the aluminum readout frames, which also stiffen the module, are attached with thermally-conducting epoxy to each of the CFC compartments. The entire $100 \mathrm{~kg}$-module is then bolted and again thermally epoxied to an aluminum strong-back. The strong-back contains alignment features as well as channels that couple into the cooling system.
Each module was installed into the $2.5 \mathrm{~cm}$-thick, $4 \mathrm{~m}$-long aluminum support cylinder, and subsequently aligned. On each of the thick annular end-flanges this cylinder contains access ports for digitizing electronics crates with associated cooling channels, as well as mounting features and alignment dowels for the forward endcap.

The endcap is constructed from 20 identical CFC modules (each with 41 crystals), individually aligned and bolted to one of two semi-circular support structures. The endcap is split vertically into two halves to facilitate access to the central detector components.

The entire calorimeter is surrounded by a double Faraday shield composed of two $1 \mathrm{~mm}$-thick aluminum sheets so that the diodes and preamplifiers are further shielded from external noise. This cage also serves as the environmental barrier, allowing the slightly hygroscopic crystals to reside in a dry, temperature controlled nitrogen atmosphere. 


\subsubsection{Cooling System}

The EMC is maintained at constant, accurately monitored temperature. Of particular concern are the stability of the photodiode leakage current which rises exponentially with temperature, and the large number of diode-crystal epoxy joints that could experience stress due to differential thermal expansion. In addition, the light yield of $\mathrm{CsI}(\mathrm{Tl})$ is weakly temperature dependent.

The primary heat sources internal to the calorimeter are the preamplifiers $(2 \times 50 \mathrm{~mW} /$ crystal $)$ and the digitizing electronics ( $3 \mathrm{~kW}$ per end-flange). In the barrel, the preamplifier heat is removed by conduction to the module strong backs which are directly cooled by Fluorinert (polychlorotrifluoro-ethylene) [81]. The digitizing electronics are housed in 80 mini-crates, each in contact with the end-flanges of the cylindrical support structure. These crates are indirectly cooled by chilled water pumped through channels milled into the end-flanges close to the inner and outer radii. A separate Fluorinert system in the endcap cools both the 20 mini-crates of digitizing electronics and the preamplifiers.

\subsection{Electronics}

The EMC electronics system, shown schematically in Figure 64, is required to have negligible impact on the energy resolution of electromagnetic showers from $20 \mathrm{MeV}$ to $9 \mathrm{GeV}$, while accommodating the use of a $6.13 \mathrm{MeV}$ radioactive source for calibration. These requirements set a limit of less than $250 \mathrm{keV}$ equivalent noise energy (ENE) per crystal and define an 18-bit effective dynamic range of the digitization scheme. For source calibrations, the least significant bit is set to $50 \mathrm{keV}$, while for colliding beam data it is set to $200 \mathrm{keV}$. To reach the required energy resolution at high energies, the coherent component has to be significantly smaller than the incoherent noise component. In addition, the impact of high rates of low energy $(<5 \mathrm{MeV})$ beam-induced photon background needs to be minimized.

\subsubsection{Photodiode Readout and Preamplifiers}

The ENE is minimized by maximizing the light yield and collection, employing a highly efficient photon detector, and a low-noise electronic readout. The PIN silicon photodiodes [82] have a quantum efficiency of $85 \%$ for the $\mathrm{CsI}(\mathrm{Tl})$ scintillation light [83]. At a depletion voltage of $70 \mathrm{~V}$, their typical dark currents were measured to be $4 \mathrm{nA}$ for an average capacitance of $85 \mathrm{pF}$; the diodes are operated at a voltage of $50 \mathrm{~V}$. The input capacitance to the preamplifier is minimized by connecting the diodes to the preamplifier with a very short cable. The preamplifier is a low-noise charge-sensitive amplifier implemented as a custom application specific integrated circuit (ASIC) [84]. It shapes the signal and acts as a band-pass filter to remove high- and lowfrequency noise components. The optimum shaping time for the $\mathrm{CsI}(\mathrm{Tl})$-photodiode readout is $2-3 \mu \mathrm{s}$, but a shorter time was chosen to reduce the probability of overlap with low-energy photons from beam background. The commensurate degradation in noise performance is recovered by implementing a realtime digital signal-processing algorithm following digitization.

To achieve the required operational reliability [85] for the inaccessible front-end readout components, two photodiodes were installed, each connected to a preamplifier. In addition, all components were carefully selected and subjected to rigorous tests, including a 72-hour burn-in of the preamplifiers at $70^{\circ} \mathrm{C}$ to avoid infant mortality. The dual signals are combined in the postamplification/digitization circuits, installed in minicrates at the end-flanges, a location that is accessible for maintenance.

\subsubsection{Postamplification, Digitization and Readout}

The two preamplifiers on each crystal, A and B, each provide amplification factors of 1 and 32 and thus reduce the dynamic range of the signal that is transmitted to the mini-crates to 13-bits. A custom auto-range encoding (CARE) circuit [84] further amplifies the signal to arrive at a total gain of $256,32,4$ or 1 for four energy ranges, $0-50 \mathrm{MeV}, \quad 50-400 \mathrm{MeV}, 0.4-3.2 \mathrm{GeV}$, and $3.2-$ 


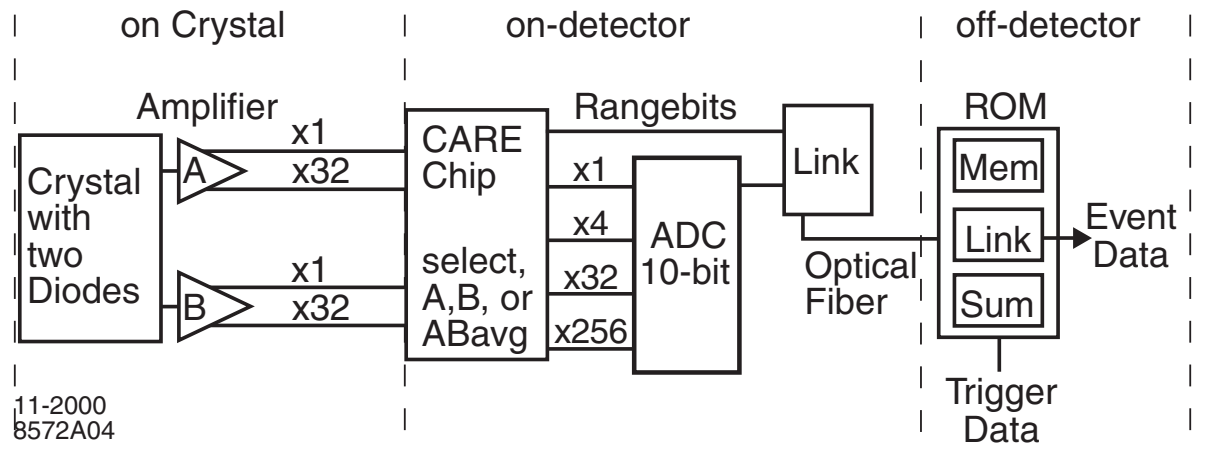

Figure 64. Schematic diagram of the EMC readout electronics.

$13.0 \mathrm{GeV}$, respectively. The appropriate range is identified by a comparator and the signal is digitized by a 10-bit, $3.7 \mathrm{MHz}$ ADC. Data from 24 crystals are multiplexed onto a fiber-optic driver and sent serially at a rate of 1.5 Gbytes/s across a 30 m-long optical fiber to the ROM. In the ROM, the continuous data stream is entered into a digital pipeline. A correction for pedestal and gain is applied to each sample. The pipeline is then tapped to extract the input to the calorimeter trigger.

Upon receipt of the L1 Accept signal, data samples within a time window of $\pm 1 \mu \mathrm{s}$ are selected for the feature extraction. Up to now, the calorimeter feature extraction algorithm performs a parabolic fit to the peak of the signal waveform to derive its energy and time. In the future, it is planned to employ a digital filter prior to the signal fit to further reduce noise. For this filter algorithm, the frequency decomposition of an average signal pulse and the typical noise spectrum are measured for all channels and subsequently used to derive an optimum set of weights that maximizes the signal-to-noise ratio. These weights are then applied to individual samples to obtain a filtered waveform.

The magnitude of the electronic noise is measured as the rms width of the pedestal distribution as shown in Figure 65. The observed distribution for all channels translates to an ENE of $230 \mathrm{keV}$ and $440 \mathrm{keV}$ with and without digital filtering; this result is comparable to design expectations. Measurements of the auto-correlation

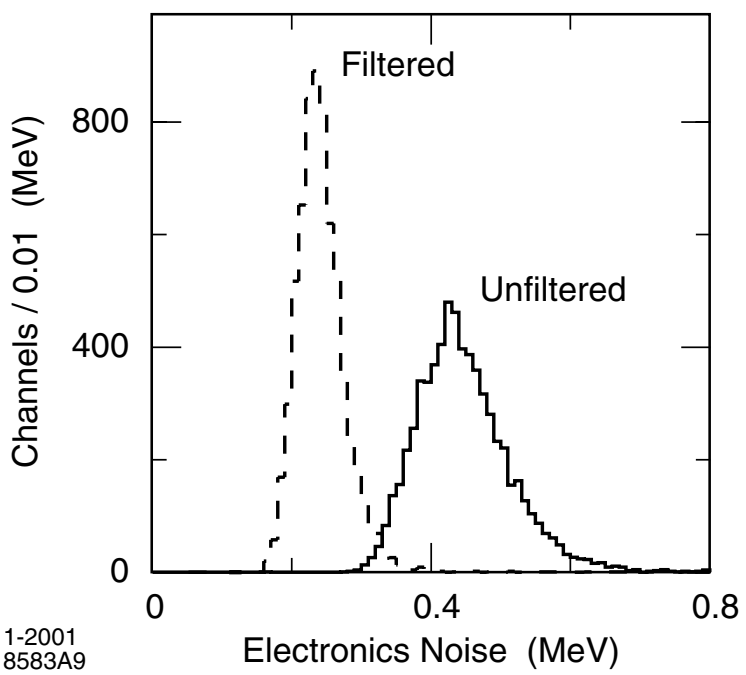

Figure 65. The distribution of equivalent noise energy (ENE) or all channels of the EMC with and without digital filtering. The data were recorded in the absence of beams by a random trigger.

function indicate that the coherent noise component is negligible compared to the incoherent noise, except for regions where the preamplifiers saturate (see below).

During data-taking, the data acquisition imposes a single-crystal readout threshold in order to keep the data volume at an acceptable level. 
This energy threshold is currently set to $1 \mathrm{MeV}$ and during stable colliding beam conditions on average 1,000 crystals are read out (measured with $600 \mathrm{~mA}$ of $e^{-}$and $1100 \mathrm{~mA}$ of $e^{+}$and a random clock trigger), corresponding to an average occupancy of $16 \%$. The electronic noise accounts for about $10 \%$, while the remaining signals originate from beam-generated background (see Section 3). A typical hadronic event contributes signals in 150 crystals.

\subsubsection{Electronics Calibration and Linearity}

To measure pedestal offsets, determine the overall gain, and to remove non-linearities the FEE are calibrated by precision charge injection into the preamplifier input. Initially, residual non-linearities of up to $12 \%$ in limited regions near each of the range changes were observed and corrected for offline [86]. These non-linearities were traced to oscillations on the ADC cards that have since been corrected. The correction resulted in markedly improved energy resolution at high energies. Residual non-linearities (typically $2-4 \%$ ) arise primarily from cross-talk, impacting both the electronics calibrations and the colliding-beam data. The effect is largest at about $630 \mathrm{MeV}(950 \mathrm{MeV})$ in a high (low) gain preamplifier channel, inducing a $2 \mathrm{MeV}(6 \mathrm{MeV})$ cross-talk signal in an adjacent channel. The implementation of an energy dependent correction is expected to significantly reduce this small, remaining effect, and lead to a further improvement of the energy resolution.

\subsubsection{Electronics Reliability}

With the exception of minor cable damage during installation (leaving two channels inoperative), the system of 13,160 readout channels has met its reliability requirements. After the replacement of a batch of failing optical-fiber drivers, the reliability of the digitizing electronics improved substantially, averaging channel losses of less than $0.1 \%$.

\subsection{Energy Calibration}

The energy calibration of the EMC proceeds in two steps. First, the measured pulse height in each crystal has to be translated to the actual en- ergy deposited. Second, the energy deposited in a shower spreading over several adjacent crystals has to be related to the energy of the incident photon or electron by correcting for energy loss mostly due to leakage at the front and the rear, and absorption in the material between and in front of the crystals, as well as shower energy not associated with the cluster.

The offline pattern recognition algorithm that groups adjacent crystals into clusters is described in detail in Section 9.6.

\subsubsection{Individual Crystal Calibration}

In spite of the careful selection and tuning of the individual crystals, their light yield varies significantly and is generally non-uniform. It also changes with time under the impact of beamgenerated radiation. The absorbed dose is largest at the front of the crystal and results in increased attenuation of the transmitted scintillation light. The light yield must therefore be calibrated at different energies, corresponding to different average shower penetration, to track the effects of the radiation damage.

The calibration of the deposited energies is performed at two energies at opposite ends of the dynamic range, and these two measurements are combined by a logarithmic interpolation. A 6.13 MeV radioactive photon source [87] provides an absolute calibration at low energy, while at higher energies $(3-9 \mathrm{GeV})$ the relation between polar angle and energy of $e^{ \pm}$from Bhabha events is exploited [88].

A flux of low-energy neutrons $\left(4 \times 10^{8} / \mathrm{s}\right)$ is used to irradiate Fluorinert [81] to produce photons of $6.13 \mathrm{MeV}$ via the reaction ${ }^{19} F+n \rightarrow{ }^{16} N+\alpha$, ${ }^{16} N \rightarrow{ }^{16} O^{*}+\beta,{ }^{16} O^{*} \rightarrow{ }^{16} O+\gamma$. The activated ${ }^{16} N$ has a half-life of 7 seconds and thus does not cause radiation damage or long-term activation. The fluid is pumped at a rate of $125 \ell / s$ from the neutron generator to a manifold of thin-walled $(0.5 \mathrm{~mm})$ aluminum pipes that are mounted immediately in front of the crystals. At this location, the typical rate of photons is $40 \mathrm{~Hz} /$ crystal.

Figure 66 shows a typical source spectrum that was derived from the raw data by employing a digital filter algorithm. For a 30-minute exposure, a statistical error of $0.35 \%$ is obtained, compared 


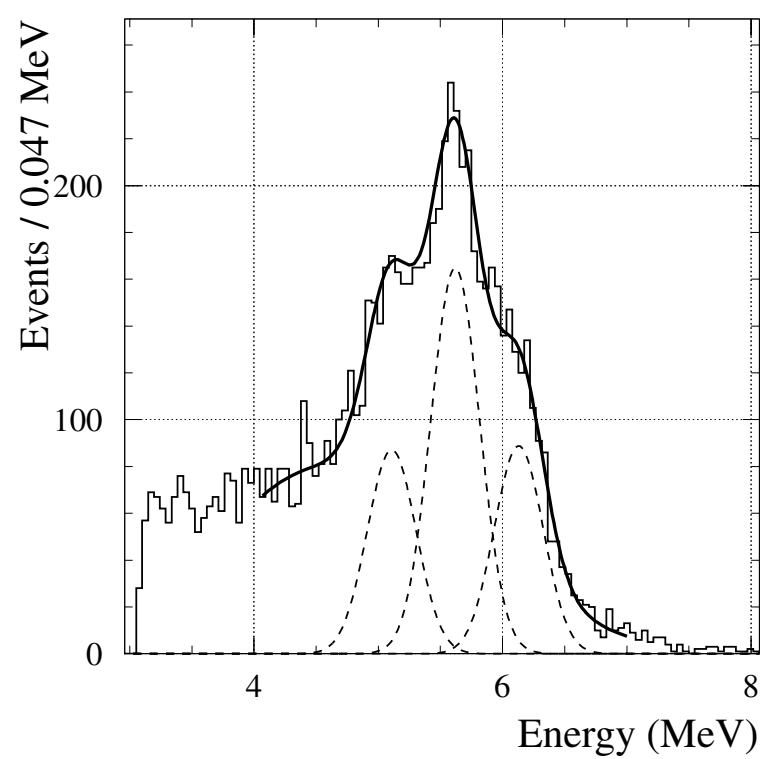

Figure 66. A typical pulse-height spectrum recorded with the radioactive source to calibrate the single-crystal energy scale of the EMC. The spectrum shows the primary $6.13 \mathrm{MeV}$ peak and two associated escape peaks at $5.62 \mathrm{MeV}$ and $5.11 \mathrm{MeV}$. The solid line represents a fit to the total spectrum, the dotted lines indicate the contributions from the three individual photon spectra.

to a systematic uncertainty of less than $0.1 \%$. This calibration is performed weekly.

At high energies, single crystal calibration is performed with a pure sample of Bhabha events [88]. As a function of the polar angle of the $e^{ \pm}$, the deposited cluster energy is constrained to equal the prediction of a GEANT-based Monte Carlo simulation [89]. For a large number of energy clusters, a set of simultaneous linear equations relates the measured to the expected energy and thus permits the determination of a gain constant for each crystal. In a 12-hour run at a luminosity of $3 \times 10^{33} \mathrm{~cm}^{-2} \mathrm{~s}^{-1}$ some $200 e^{ \pm}$per crystal can be accumulated, leading to a statistical error of $0.35 \%$. This calibration has been performed about once per month, and will be fully automated in the future.

\subsubsection{Cluster Energy Correction}

The correction for energy loss due to shower leakage and absorption is performed as a function of cluster energy and polar angle. At low energy $(\mathrm{E}<0.8 \mathrm{GeV})$, it is derived from $\pi^{0}$ decays [90]. The true energy of the photon is expressed as a product of the measured deposited energy and a correction function which depends on $\ln E$ and $\cos \theta$. The algorithm constrains the two-photon mass to the nominal $\pi^{0}$ mass and iteratively finds the coefficients of the correction function. The typical corrections are of order $6 \pm 1 \%$. The uncertainty in the correction is due to systematic uncertainties in the background estimation and the fitting technique.

At higher energy $(0.8<E<9 \mathrm{GeV})$ the correction is estimated from single-photon Monte Carlo simulations. A second technique using radiative Bhabha events [91] is being developed. The beam energy and the precise track momenta of the $e^{+}$ and $e^{-}$, together with the direction of the radiative photon, are used to fit the photon energy. This fitted value is compared to the measured photon energy to extract correction coefficients, again as a function of $\ln E$ and $\cos \theta$.

\subsection{Monitoring}

\subsubsection{Environmental Monitoring}

The temperature is monitored by 256 thermal sensors that are distributed over the calorimeter, and has been maintained at $20 \pm 0.5^{\circ} \mathrm{C}$. Dry nitrogen is circulated throughout the detector to stabilize the relative humidity at $1 \pm 0.5 \%$.

\subsubsection{Light-Pulser System}

The light response of the individual crystals is measured daily using a light-pulser system [92, 93]. Spectrally filtered light from a xenon flash lamp is transmitted through optical fibers to the rear of each crystal. The light pulse is similar in spectrum, rise-time and shape to the scintillation light in the $\mathrm{CsI}(\mathrm{Tl})$ crystals. The pulses are varied in intensity by neutral-density filters, allowing a precise measurement of the linearity of light collection, conversion to charge, amplification, and digitization. The intensity is monitored pulse-topulse by comparison to a reference system with two radioactive sources, ${ }^{241} \mathrm{Am}$ and ${ }^{148} \mathrm{Gd}$, that 


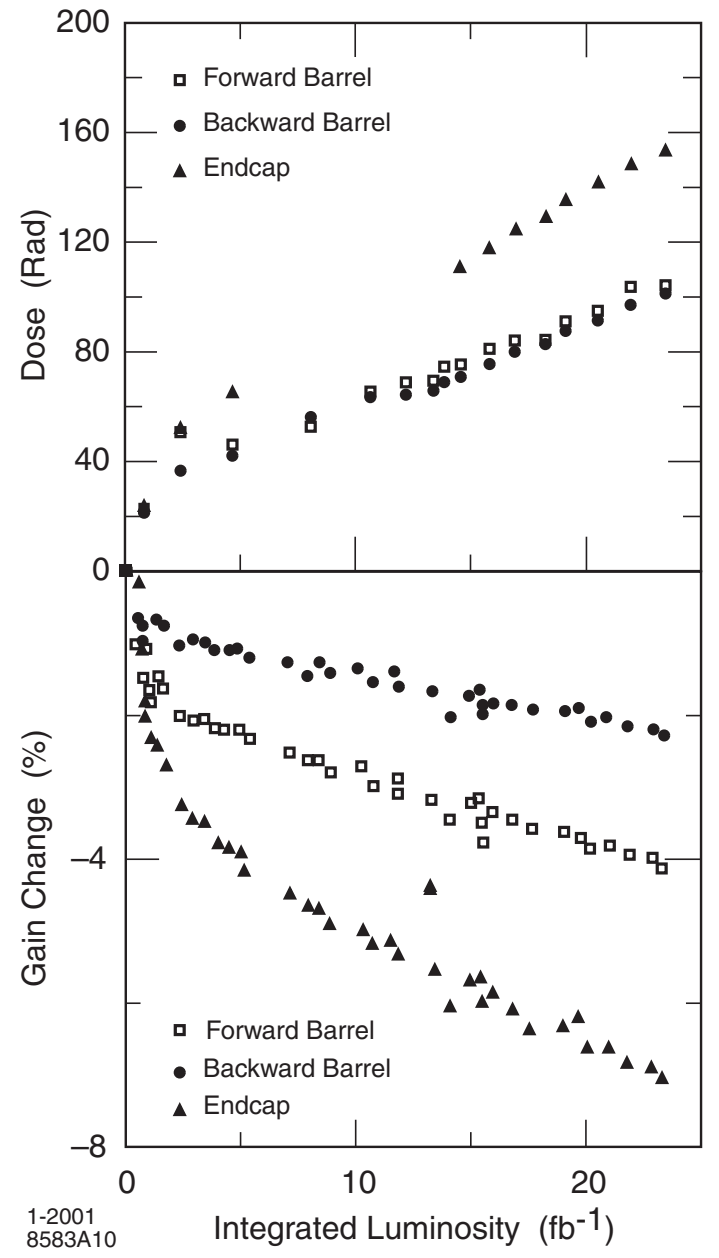

Figure 67. Impact of beam-generated radiation on the $\mathrm{CsI}(\mathrm{Tl})$ crystals: a) the integrated dose measured with RadFETs placed in front of the crystals, b) the degradation in light yield measured with the radioactive-source calibration system.

are attached to a small $\mathrm{CsI}(\mathrm{Tl})$ crystal that is read out by both a photodiode and a photomultiplier tube. The system is stable to $0.15 \%$ over a period of one week and has proven to be very valuable in diagnosing problems. For example, the ability to accurately vary the light intensity led to the detection of non-linear response in the electronics [92].

\subsubsection{Radiation Monitoring and Damage}

The radiation exposure is monitored by 56 and 60 realtime integrating dosimeters (RadFETs) [18] placed in front of the barrel and endcap crystals. In Figure 67, the accumulated dose is compared to the observed loss in scintillation light, separately for the endcap, the forward, and the backward barrel. The dose appears to follow the integrated luminosity, approximately linearly. The light loss is greatest in the forward region corresponding to the area of highest integrated radiation dose. The size of the observed light loss is close to expectations, based on extensive irradiation tests.

\subsection{Reconstruction Algorithms}

A typical electromagnetic shower spreads over many adjacent crystals, forming a cluster of energy deposits. Pattern recognition algorithms have been developed to efficiently identify these clusters and to differentiate single clusters with one energy maximum from merged clusters with more than one local energy maximum, referred to as a bumps. Furthermore, the algorithms determine whether a bump is generated by a charged or a neutral particle.

Clusters are required to contain at least one seed crystal with an energy above $10 \mathrm{MeV}$. Surrounding crystals are considered as part of the cluster if their energy exceeds a threshold of $1 \mathrm{MeV}$, or if they are contiguous neighbors (including corners) of a crystal with at least $3 \mathrm{MeV}$. The value of the single crystal threshold is set by the data acquisition system in order to keep the data volume at an acceptable level, given the current level of electronics noise and beam-generated background. It is highly desirable to reduce this threshold since fluctuations in the effective energy loss at the edges of a shower cause a degradation in resolution, particularly at low energies.

Local energy maxima are identified within a cluster by requiring that the candidate crystal have an energy, $E_{\text {LocalMax }}$, which exceeds the energy of each of its neighbors, and satisfy the following condition: $0.5(N-2.5)>$ $E_{\mathrm{NMax}} / E_{\text {LocalMax }}$, where $E_{\mathrm{NMax}}$ is the highest energy of any of the neighboring $N$ crystals with an energy above $2 \mathrm{MeV}$. 
Clusters are divided into as many bumps as there are local maxima. An iterative algorithm is used to determine the energy of the bumps. Each crystal is given a weight, $w_{i}$, and the bump energy is defined as $E_{\text {bump }}=\sum_{i} w_{i} E_{i}$, where the sum runs over all crystals in the cluster. For a cluster with a single bump, the result is $w_{i} \equiv 1$. For a cluster with multiple bumps, the crystal weight for each bump is calculated as

$w_{i}=E_{i} \frac{\exp \left(-2.5 r_{i} / r_{M}\right)}{\sum_{j} E_{j} \exp \left(-2.5 r_{j} / r_{M}\right)}$,

where the index $j$ runs over all crystals in the cluster. $r_{M}$ refers to the Molière radius, and $r_{i}$ is the distance of the $i$ th crystal from the centroid of the bump. At the outset, all weights are set to one. The process is then iterated, whereby the centroid position used in calculating $r_{i}$ is determined from the weights of the previous iteration, until the bump centroid position is stable to within a tolerance of $1 \mathrm{~mm}$.

The position of a bump is calculated using a center-of-gravity method with logarithmic, rather than linear weights $[94,95], W_{i}=4.0+$ $\ln E_{i} / E_{\text {bump }}$, where only crystals with positive weights, i.e., $E_{i}>0.0184 \times E_{\text {bump }}$, are used in the calculation. This procedure emphasizes lowerenergy crystals, while utilizing only those crystals that make up the core of the cluster. A systematic bias of the calculated polar angle originates from the non-projectivity of the crystals. This bias is corrected by a simple offset of $-2.6 \mathrm{mrad}$ for $\theta>90^{\circ}$ and $+2.6 \mathrm{mrad}$ for $\theta<90^{\circ}$.

A bump is associated with a charged particle by projecting a track to the inner face of the calorimeter. The distance between the track impact point and the bump centroid is calculated, and if it is consistent with the angle and momentum of the track, the bump is associated with this charged particle. Otherwise, it is assumed to originate from a neutral particle.

On average, 15.8 clusters are detected per hadronic event, of which 10.2 are not associated with charged particle tracks. At current operating conditions, beam-induced background contributes on average 1.4 neutral clusters with energies above $20 \mathrm{MeV}$. This number is significantly smaller than the average number of crystals with

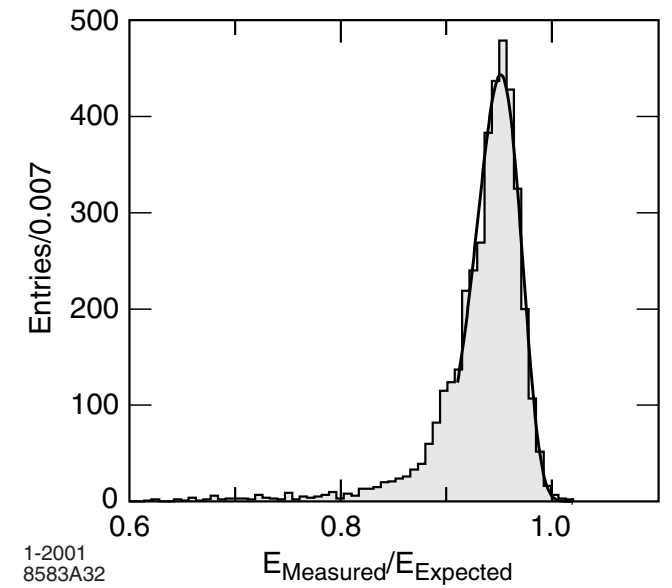

Figure 68. The ratio of the EMC measured energy to the expected energy for electrons from Bhabha scattering of $7.5 \mathrm{GeV} / c$. The solid line indicates a fit using a logarithmic function.

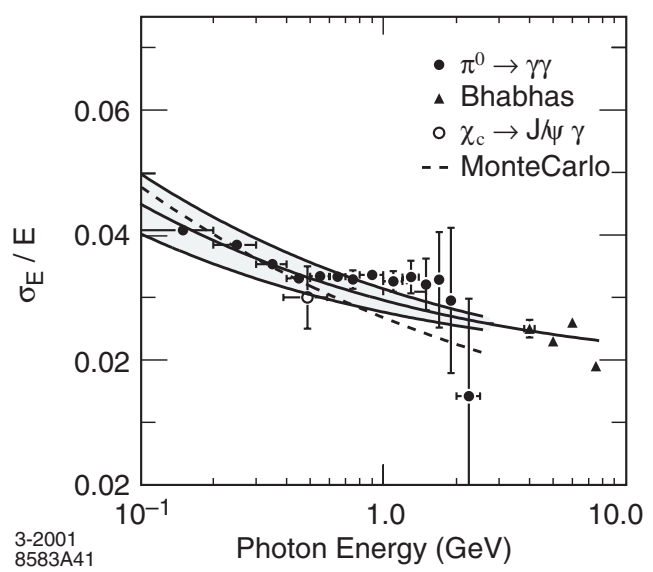

Figure 69. The energy resolution for the ECM measured for photons and electrons from various processes. The solid curve is a fit to Equation 6 and the shaded area denotes the rms error of the fit.

energies above $10 \mathrm{MeV}$ (see Section 3).

\subsection{Performance}

\subsubsection{Energy Resolution}

At low energy, the energy resolution of the EMC is measured directly with the radioactive 


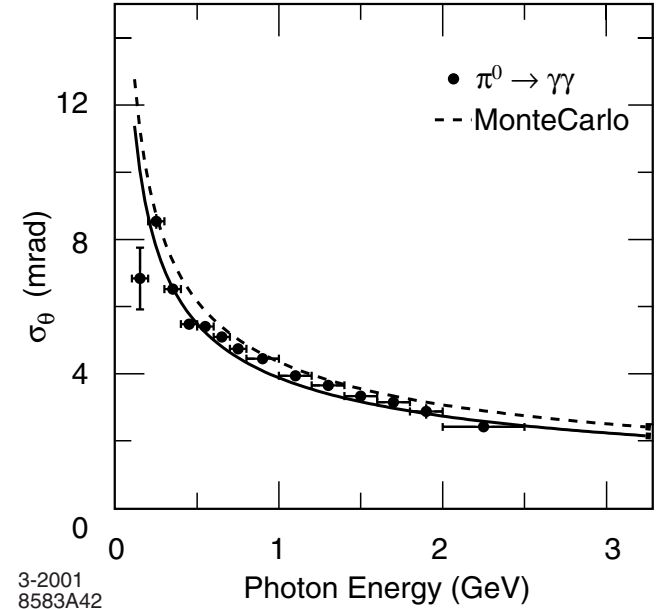

Figure 70. The angular resolution of the EMC for photons from $\pi^{0}$ decays. The solid curve is a fit to Equation 7.

source yielding $\sigma_{E} / E=5.0 \pm 0.8 \%$ at $6.13 \mathrm{MeV}$ (see Figure 66). At high energy, the resolution is derived from Bhabha scattering, where the energy of the detected shower can be predicted from the polar angle of the $e^{ \pm}$. The measured resolution is $\sigma_{E} / E=1.9 \pm 0.07 \%$ at $7.5 \mathrm{GeV}$ (see Figure 68). Figure 69 shows the energy resolution extracted from a variety of processes as a function of energy. Below $2 \mathrm{GeV}$, the mass resolution of $\pi^{0}$ and $\eta$ mesons decaying into two photons of approximately equal energy is used to infer the EMC energy resolution [90]. The decay $\chi_{c 1} \rightarrow J / \psi \gamma$ provides a measurement at an average energy of about $500 \mathrm{MeV}$, and measurements at high energy are derived from Bhabha scattering. A fit to the energy dependence results in

$\frac{\sigma_{E}}{E}=\frac{(2.32 \pm 0.30) \%}{\sqrt[4]{E(\mathrm{GeV})}} \oplus(1.85 \pm 0.12) \%$.

Values of these fitted parameters are higher than the somewhat optimistic design expectations, but they agree with detailed Monte Carlo simulations which include the contributions from electronic noise and beam background, as well as the impact of the material and the energy thresholds.

\subsubsection{Angular Resolution}

The measurement of the angular resolution is based on the analysis of $\pi^{0}$ and $\eta$ decays to two

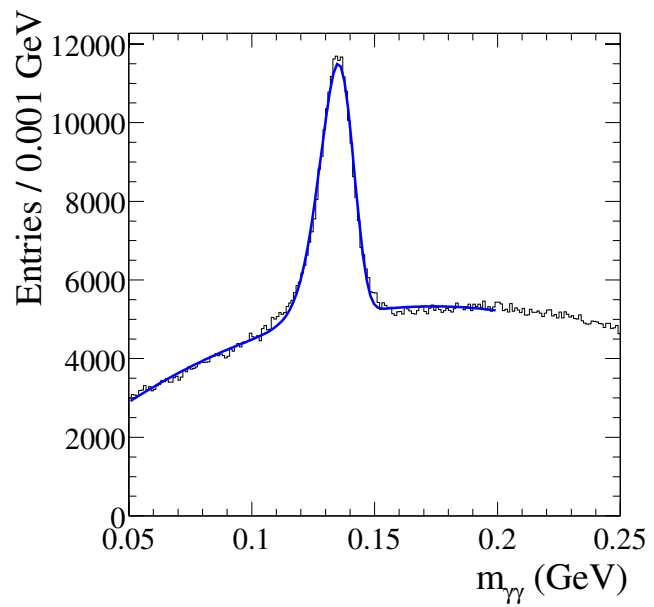

Figure 71. Invariant mass of two photons in $B \bar{B}$ events. The energies of the photons and the $\pi^{0}$ are required to exceed $30 \mathrm{MeV}$ and $300 \mathrm{MeV}$, respectively. The solid line is a fit to the data.

photons of approximately equal energy. The result is presented in Figure 70. The resolution varies between about $12 \mathrm{mrad}$ at low energies and $3 \mathrm{mrad}$ at high energies. A fit to an empirical parameterization of the energy dependence results in

$$
\begin{aligned}
\sigma_{\theta} & =\sigma_{\phi} \\
& =\left(\frac{3.87 \pm 0.07}{\sqrt{E(\mathrm{GeV})}}+0.00 \pm 0.04\right) \mathrm{mrad}
\end{aligned}
$$

These fitted values are slightly better than would be expected from detailed Monte Carlo simulations.

\subsection{3. $\pi^{0}$ Mass and Width}

Figure 71 shows the two-photon invariant mass in $B \bar{B}$ events. The reconstructed $\pi^{0}$ mass is measured to be $135.1 \mathrm{MeV} / c^{2}$ and is stable to better than $1 \%$ over the full photon energy range. The width of $6.9 \mathrm{MeV} / c^{2}$ agrees well with the prediction obtained from detailed Monte-Carlo simulations. In low-occupancy $\tau^{+} \tau^{-}$events, the width is slightly smaller, $6.5 \mathrm{MeV} / c^{2}$, for $\pi^{0}$ energies below $1 \mathrm{GeV}$. A similar improvement is also observed in analyses using selected isolated photons in hadronic events. 


\subsubsection{Electron Identification}

Electrons are separated from charged hadrons primarily on the basis of the shower energy, lateral shower moments, and track momentum. In addition, the $d E / d x$ energy loss in the DCH and the DIRC Cherenkov angle are required to be consistent with an electron. The most important variable for the discrimination of hadrons is the ratio of the shower energy to the track momentum $(E / p)$. Figure 72 shows the efficiency for electron identification and the pion misidentification probability as a function of momentum for two sets of selection criteria. The electron efficiency is measured using radiative Bhabhas and $e^{+} e^{-} \rightarrow e^{+} e^{-} e^{+} e^{-}$events. The pion misidentification probability is measured for selected charged pions from $K_{S}^{0}$ decays and threeprong $\tau$ decays. A tight (very tight) selector results in an efficiency plateau at $94.8 \%(88.1 \%)$ in the momentum range $0.5<p<2 \mathrm{GeV} / c$. The pion misidentification probability is of order $0.3 \%$ $(0.15 \%)$ for the tight (very tight) selection criteria.

\subsection{Summary}

The EMC is presently performing close to design expectations. Improvements in the energy resolution are expected from the optimization of the feature-extraction algorithms designed to further reduce the electronics noise. Modifications to the electronics should allow for more precise calibrations. The expected noise reduction should permit a lower single-crystal readout threshold. However, this decrease in noise might be offset by an increase in the beam background that is expected for higher luminosities and beam currents.

\section{Detector for Muons and Neutral Hadrons}

\subsection{Physics Requirements and Goals}

The Instrumented Flux Return (IFR) was designed to identify muons with high efficiency and good purity, and to detect neutral hadrons (primarily $K_{L}^{0}$ and neutrons) over a wide range of momenta and angles. Muons are important for tagging the flavor of neutral $B$ mesons via semi-
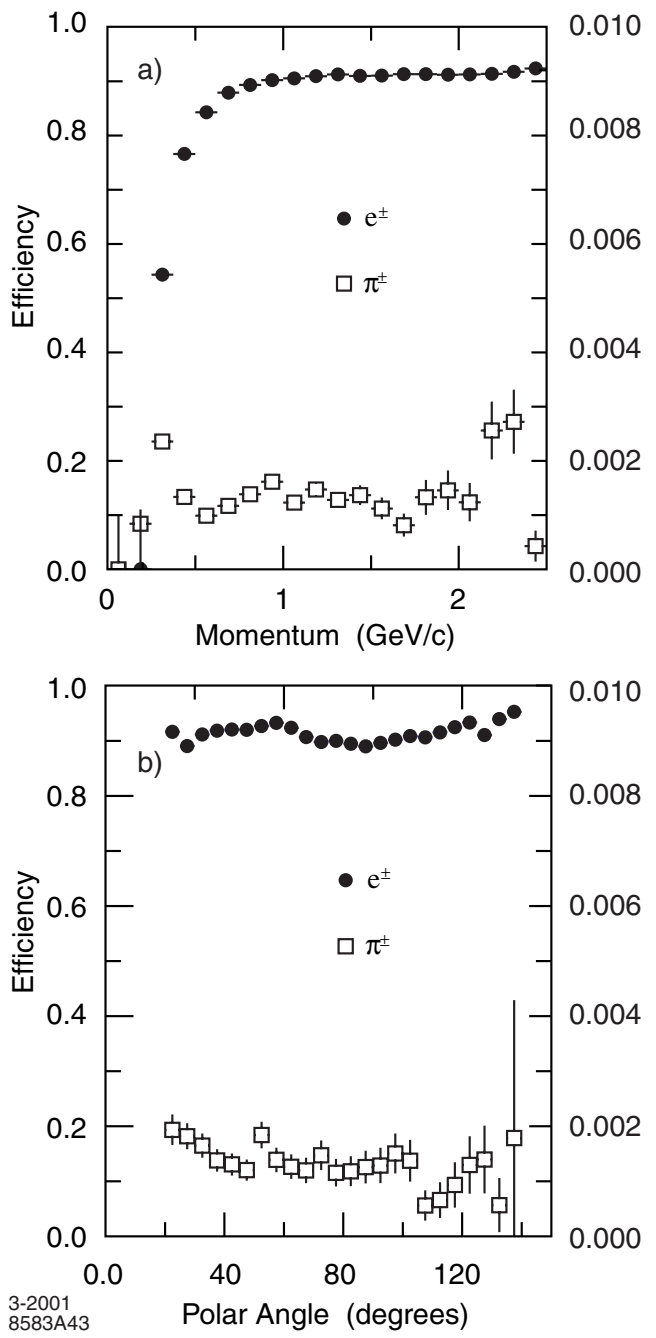

Figure 72. The electron efficiency and pion misidentification probability as a function of a) the particle momentum and b) the polar angle, measured in the laboratory system.

leptonic decays, for the reconstruction of vector mesons, like the $J / \psi$, and for the study of semileptonic and rare decays involving leptons of $B$ and $D$ mesons and $\tau$ leptons. $K_{L}^{0}$ detection allows the study of exclusive $B$ decays, in particular $C P$ eigenstates. The IFR can also help in vetoing charm decays and improve the reconstruction of neutrinos.

The principal requirements for IFR are large solid angle coverage, good efficiency, and high 
background rejection for muons down to momenta below $1 \mathrm{GeV} / c$. For neutral hadrons, high efficiency and good angular resolution are most important. Because this system is very large and difficult to access, high reliability and extensive monitoring of the detector performance and the associated electronics plus the voltage distribution are required.

\subsection{Overview and RPC Concept}

The IFR uses the steel flux return of the magnet as a muon filter and hadron absorber. Single gap resistive plate chambers (RPCs) [96] with two-coordinate readout have been chosen as detectors.

The RPCs are installed in the gaps of the finely segmented steel (see Section 4) of the barrel and the end doors of the flux return, as illustrated in Figure 73. The steel segmentation has been chosen on the basis of Monte Carlo studies of muon penetration and charged and neutral hadron interactions. The steel is segmented into 18 plates, increasing in thickness from $2 \mathrm{~cm}$ for the inner nine plates to $10 \mathrm{~cm}$ for the outermost plates. The nominal gap between the steel plates is $3.5 \mathrm{~cm}$ in the inner layers of the barrel and $3.2 \mathrm{~cm}$ elsewhere. There are 19 RPC layers in the barrel and 18 in the endcaps. In addition, two layers of cylindrical RPCs are installed between the EMC and the magnet cryostat to detect particles exiting the EMC.

RPCs detect streamers from ionizing particles via capacitive readout strips. They offer several advantages: simple, low cost construction and the possibility of covering odd shapes with minimal dead space. Further benefits are large signals and fast response allowing for simple and robust frontend electronics and good time resolution, typically 1-2 ns. The position resolution depends on the segmentation of the readout; a value of a few $\mathrm{mm}$ is achievable.

The construction of the planar and cylindrical RPCs differ in detail, but they are based on the same concept. A cross section of an RPC is shown schematically in Figure 74.

The planar RPCs consist of two bakelite (phenolic polymer) sheets, $2 \mathrm{~mm}$-thick and separated by a gap of $2 \mathrm{~mm}$. The gap is enclosed at the

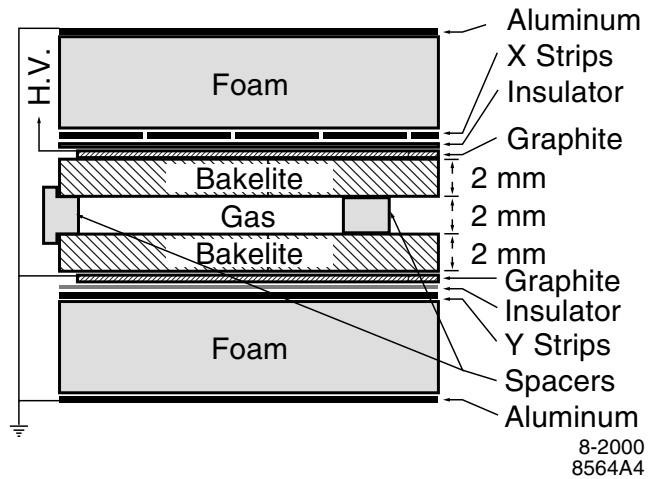

Figure 74. Cross section of a planar RPC with the schematics of the high voltage (HV) connection.

edge by a $7 \mathrm{~mm}$ wide frame. The gap width is kept uniform by polycarbonate spacers $\left(0.8 \mathrm{~cm}^{2}\right)$ that are glued to the bakelite, spaced at distances of about $10 \mathrm{~cm}$. The bulk resistivity of the bakelite sheets has been especially tuned to $10^{11}-10^{12} \Omega \mathrm{cm}$. The external surfaces are coated with graphite to achieve a surface resistivity of $\sim$ $100 \mathrm{k} \Omega$ /square. These two graphite surfaces are connected to high voltage $(\sim 8 \mathrm{kV})$ and ground, and protected by an insulating mylar film. The bakelite surfaces facing the gap are treated with linseed oil. The RPCs are operated in limited streamer mode and the signals are read out capacitively, on both sides of the gap, by external electrodes made of aluminum strips on a mylar substrate.

The cylindrical RPCs have resistive electrodes made of a special plastic composed of a conducting polymer and ABS plastic. The gap thickness and the spacers are identical to the planar RPCs. No linseed oil or any other surface treatments have been applied. The very thin and flexible electrodes are laminated to fiberglass boards and foam to form a rigid structure. The copper readout strips are attached to the fiberglass boards.

\subsection{RPC Design and Construction}

The IFR detectors cover a total active area of about $2,000 \mathrm{~m}^{2}$. There are a total of $806 \mathrm{RPC}$ modules, 57 in each of the six barrel sectors, 108 in each of the four half end doors, and 32 in the two cylindrical layers. The size and the shape of 


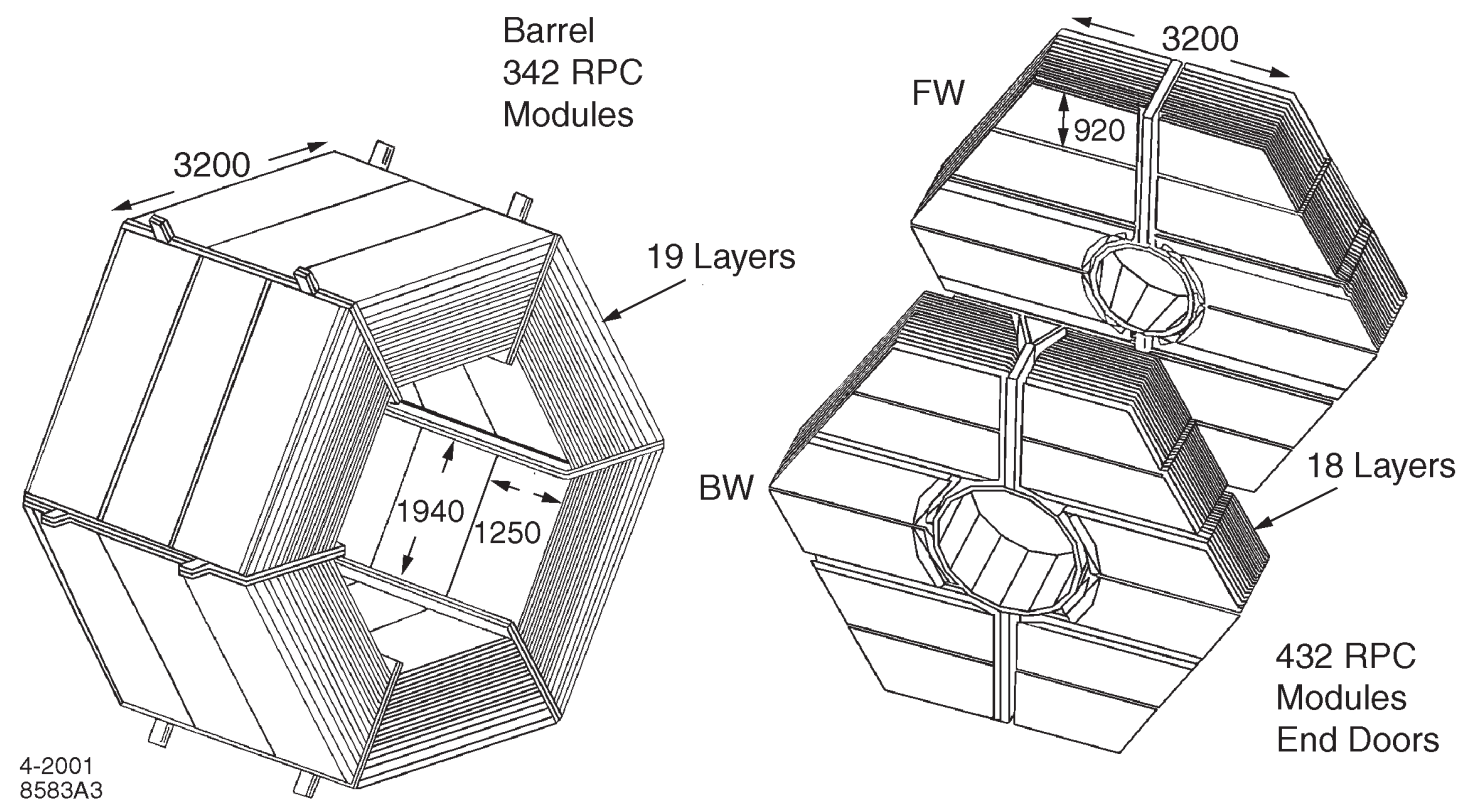

Figure 73. Overview of the IFR: Barrel sectors and forward (FW) and backward (BW) end doors; the shape of the RPC modules and their dimensions are indicated.

the modules are matched to the steel dimensions with very little dead space. More than 25 different shapes and sizes were built. Because the size of a module is limited by the maximum size of the material available, i.e., $320 \times 130 \mathrm{~cm}^{2}$ for the bakelite sheets, two or three RPC modules are joined to form a gap-size chamber. The modules of each chamber are connected to the gas system in series, while the high voltage is supplied separately to each module.

In the barrel sectors, the gaps between the steel plates extend $375 \mathrm{~cm}$ in the $z$ direction and vary in width from $180 \mathrm{~cm}$ to $320 \mathrm{~cm}$. Three modules are needed to cover the whole area of the gap, as shown in Figure 73. Each barrel module has 32 strips running perpendicular to the beam axis to measure the $z$ coordinate and 96 strips in the orthogonal direction extending over three modules to measure $\phi$.

Each of the four half end doors is divided into three sections by steel spacers that are needed for mechanical strength. Each of these sections is covered by two RPC modules that are joined to form a larger chamber with horizontal and verti- cal readout strips.

The readout strips are separated from the ground aluminum plane by a $4 \mathrm{~mm}$-thick foam sheet and form strip lines of $33 \Omega$ impedance. The strips are connected to the readout electronics at one end and terminated with a $2 \mathrm{k} \Omega$ resistor at the other. Even and odd numbered strips are connected to different front-end cards (FECs), so that a failure of a card does not result in a total loss of signal, since a particle crossing the gap typically generates signals in two or more adjacent strips.

The cylindrical RPC is divided into four sections, each covering a quarter of the circumference. Each of these sections has four sets of two single gap RPCs with orthogonal readout strips, the inner with helical $u-v$ strips that run parallel to the diagonals of the module, and the outer with strips parallel to $\phi$ and $z$. Within each section, the strips of the four sets of RPCs in a given readout plane are connected to form long strips extending over the whole chamber. Details of the segmentation and dimensions can be found in Table 13 . 
Table 13

IFR Readout segmentation. The total number of channels is close to 53,000 .

\begin{tabular}{cccccccr}
\hline \hline section & $\begin{array}{c}\text { \# of } \\
\text { sectors }\end{array}$ & coordinate & $\begin{array}{c}\text { \# of readout } \\
\text { layers }\end{array}$ & $\begin{array}{c}\text { \# strips } \\
\text { layer/sect }\end{array}$ & $\begin{array}{c}\text { strip length } \\
(\mathrm{cm})\end{array}$ & $\begin{array}{c}\text { strip width } \\
(\mathrm{mm})\end{array}$ & $\begin{array}{r}\text { total \# } \\
\text { channels }\end{array}$ \\
\hline barrel & 6 & $\phi$ & 19 & 96 & 350 & $19.7-32.8$ & $\approx 11,000$ \\
& & $\mathrm{Z}$ & 19 & 96 & $190-318$ & 38.5 & $\approx 11,000$ \\
endcap & \multirow{4}{*}{4} & $\mathrm{y}$ & 18 & $6 \mathrm{x} 32$ & $124-262$ & 28.3 & 13,824 \\
& & $\mathrm{x}$ & 18 & $3 \times 64$ & $10-180$ & 38.0 & $\approx 15,000$ \\
cylinder & \multirow{4}{*}{4} & $\phi$ & 1 & 128 & 370 & 16.0 & 512 \\
& & $\mathrm{Z}$ & 1 & 128 & 211 & 29.0 & 512 \\
& & $\mathrm{u}$ & 1 & 128 & $10-422$ & 29.0 & 512 \\
& & $\mathrm{u}$ & 1 & 128 & $10-423$ & 29.0 & 512 \\
\hline \hline
\end{tabular}

Prior to shipment to SLAC, all RPC modules were tested with cosmic rays. The single rates, dark currents, and efficiency were measured as a function of HV. In addition, detailed studies of the efficiency, spatial resolution, and strip multiplicity were performed $[97,98]$.

After the assembly of RPC modules into gapsize chambers, a new series of cosmic rays tests was performed to assure stable and efficient operation. Before the installation of the steel flux return, the planar chambers were inserted into the gaps. The cylindrical chambers were inserted after the installation of the solenoid and the EMC.

For each module, test results and conditions are retained in a database, together with records of the critical parameters of the components, the assembly and cabling. In addition, operational data are stored, such as the results of the weekly efficiency measurements that are used in the reconstruction and simulation software.

\subsection{Power and Utilities}

Once the return flux assembly was completed, the FECs [99] were installed and the low (LV) and high voltage $(\mathrm{HV})$, and the gas system were connected. There are approximately 3,300 FECs, most placed inside the steel gaps, while the remainder was installed in custom crates mounted on the outside of the steel.

Each FEC is individually connected to the LV power distribution. The total power required by the entire system is about $8 \mathrm{~kW}$ at $+7.0 \mathrm{~V}$ and $2.5 \mathrm{~kW}$ at $-5.2 \mathrm{~V}$. The $\mathrm{LV}$ power is supplied by custom-built switching devices with load and line regulation of better than $1 \%$. Additional features are precision shunts to measure output currents and TTL logic to inhibit output.

The HV power system is a custom adaptation by CAEN [100]. Each HV mainframe can hold up to ten cards, each carrying two independent $10 \mathrm{kV}$ outputs at $1 \mathrm{~mA}$ and $2 \mathrm{~mA}$. The RPC modules are connected via a distribution box to the HV supplies. Each distribution box services six RPC modules and up to six distribution boxes are daisy-chained to one HV output. Provisions are made for monitoring the currents drawn by each module. To reduce noise, the RPC ground plane is decoupled from the HV power supply ground by a $100 \mathrm{k} \Omega$ resistor.

The RPCs operate with a non-flammable gas mixture, typically $56.7 \%$ Argon, $38.8 \%$ Freon 134a (1,1,1,2 tetrafluoroethane), and $4.5 \%$ isobutane. This mixture is drawn from a 760 liter tank that is maintained at an absolute pressure of 1500-1600 Torr. The mixing tank is filled on demand with the three component gases under control of mass-flow meters. Samples are extracted from the mixing tank periodically and analyzed to verify the correct mixture.

The mixed gas is distributed at a gauge pressure of approximately 6.5 Torr through a parallel manifold system of $12.7 \mathrm{~mm}$-diameter copper tubing. Each chamber is connected to the manifold through several meters of $6 \mathrm{~mm}$-diameter plastic tubing (polyamide or Teflon). The flow to each of 


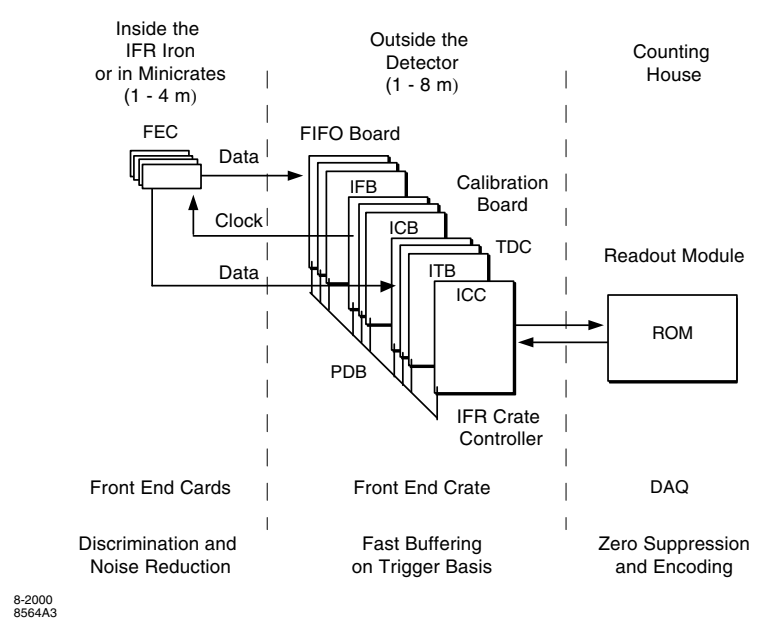

Figure 75 . Block diagram of the IFR electronics.

these is adjusted individually with a small multiturn metering valve. Protection against overpressure is provided by an oil bubbler to atmosphere in parallel with each chamber, limiting the gauge pressure in the chamber to a maximum of about 1 Torr. Return flow of gas from each chamber is monitored by a second oil bubbler which creates a back pressure of about 0.2 Torr. The total flow through the entire system is approximately $5 \ell /$ minute and corresponds on average to two gas exchanges per day.

\subsection{Electronics}

A block diagram of the IFR electronics system [101] is shown in Figure 75. It includes the FECs, the data acquisition, and the trigger.

The FECs service 16 channels each. They shape and discriminate the input signals and set a bit for each strip with a signal above a fixed threshold. The input stage operates continuously and is connected directly to the strips which act as transmission lines. A fast OR of all FEC input signals provides time information and is also used for diagnostic purposes. Two types of FECs are employed to handle inputs of different polarity for signals from the opposite sides of the gap. Because of the very low occupancy there is no provision for buffering during the trigger latency [99].
Signals from 3,300 FECs are transmitted to eight custom IFR front-end crates that are located near the detector. Each front-end crate houses up to 16 data handling cards, four trigger cards and a crate controller card (ICC) that collects data from the DAQ cards and forwards them to a ROM. There are three kinds of data cards: the FIFO boards (IFBs) that buffer strip hits, the TDC boards (ITBs) that provide time information, and the calibration boards (ICBs) that inject test pulses into the FECs. To deliver the data and clock signals to all the boards in the front-end crate, a custom backplane (PDB) for the standard $6 \mathrm{U}$ Eurocard crate was designed using 9-layer strip line technology. Each board is connected to the ICC via three point-to-point lines for three single-end signals (data-in, data-out and clock), all of the same length and impedance $(50 \Omega)$.

The IFB reads the digital hit patterns from the FECs in less than $2.2 \mathrm{~ms}$, stores the data into FIFOs and transfers the FIFO contents into one of the ROMs. Each IFB handles 64 FECs, acting as an acquisition master. It receives commands via the $\mathrm{PDB}$, and transmits and receives data patterns from the ROM (via G-Link and ICC). This card operates with the system clock frequency of $59.5 \mathrm{MHz}$.

The ICB is used for front-end tests and calibrations. A signal with programmable amplitude and width is injected into the FEC input stage. To provide timing calibration and to determine the correct readout delay, the board is also used together with the TDCs.

The ICC interfaces the crate backplane with the G-Link. The physical interface is the Finisar transceiver, a low cost and highly reliable data link for applications up to 1.5 Gbytes/s.

The TDC boards exploit the excellent time resolution of the RPCs. Each board has 96 ECL differential input channels for the fast OR signals from the FECs. Time digitization is achieved by three custom TDCs, designed at CERN [102]. Upon receipt of a L1 Accept, data are selected and stored until readout by the ROM. The $59.5 \mathrm{MHz}$ clock signal is synchronized with the data and distributed to the 16 boards. High performance drivers provide a reliable clock distribution with a jitter of less than $0.5 \mathrm{~ns}$. 


\subsection{Slow Controls and Online Monitoring}

The IFR is a system with a large number of components and electronics distributed all over the BABAR detector. To assure safe and stable operation, an extensive monitoring and control system was installed. The IFR Online Detector Control (IODC) monitors the performance of the RPCs by measuring the singles counting rate and the dark current of every module. It also controls and monitors the operation of the electronics, the $\mathrm{DAQ}$ and trigger, as well as the $\mathrm{LV}$, the $\mathrm{HV}$, and the gas system. The total number of hardware channels is close to 2,500 [103].

The system has been easy to operate. HV trips are rare. Temperature monitoring in the steel structure and the electronics crates has proven very useful for the diagnosis of operational problems. The occupancy is extremely low everywhere, except in layer 18 of the forward end door which lacks adequate shielding from machinegenerated background. On average, there are about 100-150 strip signals per event.

\subsection{Efficiency Measurements and Performance}

The efficiency of the RPCs is evaluated both for normal collision data and for cosmic ray muons recorded with the IFR trigger. Every week, cosmic ray data are recorded at different voltage settings and the efficiency is measured chamber-bychamber as a function of the applied voltage. The absolute efficiency at the nominal working voltage (typically $7.6 \mathrm{kV}$ ) is stored in the database for use in the event reconstruction software.

To calculate the efficiency in a given chamber, nearby hits in a given layer and hits in different layers are combined to form clusters. Two different algorithms are used. The first is based solely on the IFR information and uses data recorded with a dedicated IFT trigger; the second matches the IFR clusters with the tracks reconstructed in the DCH. Both these algorithms start from onedimensional IFR clusters defined as a group of adjacent hits in one of the two readout coordinates. The cluster position is defined as the centroid of the strips in the cluster. In the first algorithm, two-dimensional clusters are formed by joining

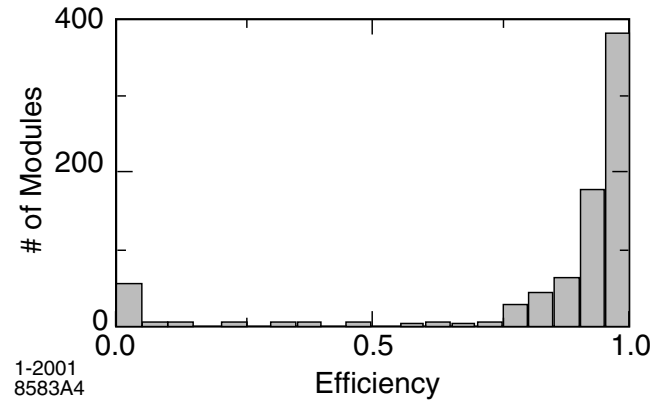

Figure 76. Distribution of the efficiency for all RPC modules measured with cosmic rays in June 1999. Some 50 modules were not operational at that time.

one-dimensional clusters (of the same readout coordinate) in different layers. In each sector, two-dimensional clusters in different coordinates are combined into three-dimensional clusters provided there are fewer than three layers missing in one of the two coordinates. The second algorithm extrapolates charged tracks reconstructed by the DCH. IFR clusters which are less than $12 \mathrm{~cm}$ from the extrapolated track are combined to form three-dimensional or two-dimensional clusters. A detailed discussion of the clustering algorithm can be found elsewhere [104].

The residual distributions from straight line fits to two-dimensional clusters typically have an rms width of less than $1 \mathrm{~cm}$. An RPC is considered efficient if a signal is detected at a distance of less than $10 \mathrm{~cm}$ from the fitted straight line in either of the two readout planes. Following the installation and commissioning of the IFR system, all RPC modules were tested with cosmic rays and their efficiency was measured. The results are presented in Figure 76. Of the active RPC modules, $75 \%$ exceed an efficiency of $90 \%$.

Early tests indicated that the RPC dark current was very temperature dependent, specifically, the current increases $14-20 \%$ per ${ }^{\circ} \mathrm{C}$. Because the IR experimental hall does not have temperature regulation this presents a serious problem. The FECs that are installed in the steel gaps dissipate $3 \mathrm{~W}$ each, generating a total power of $3.3 \mathrm{~kW}$ in the barrel and $1.3 \mathrm{~kW}$ in the forward end door. 


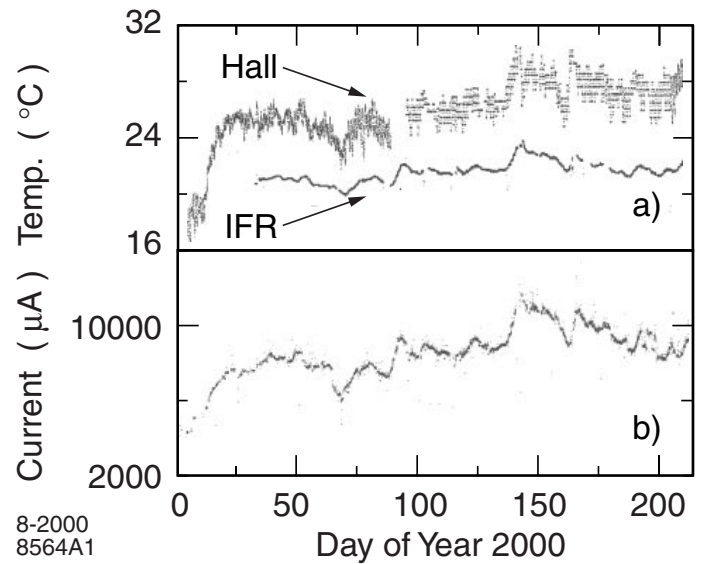

Figure 77. History of the temperature and dark current in the RPC modules since January 2000. a) temperature in the IR-2 hall and in the backward end door; b) total dark current in the 216 modules of the backward end door.

During the first summer of operation, the daily average temperature in the IR hall was $28^{\circ} \mathrm{C}$ and the maximum hall temperature frequently exceeded $31^{\circ} \mathrm{C}$. The temperature inside the steel rose to more than $37^{\circ} \mathrm{C}$ and the dark currents in many modules exceeded the capabilities of the HV system and some RPCs had to be temporarily disconnected.

To overcome this problem, water cooling was installed on the barrel and end door steel, removing $\approx 10 \mathrm{~kW}$ of heat and stabilizing the temperature at $20-21^{\circ} \mathrm{C}$ in the barrel, $22^{\circ} \mathrm{C}$ in the backward and $24^{\circ} \mathrm{C}$ in the forward end doors. Figure 77 shows the history of temperature in the hall and temperature and total dark current in the backward end door. While the current closely follows the temperature variations, the range of change is now limited to a few degrees.

During operation at high temperatures, a large fraction of the RPCs (>50\%) showed not only very high dark currents, but also some reduction in efficiency compared to earlier measurements [105]. After the cooling was installed and the RPCs were reconnected, some of them continued to deteriorate while others remained stable, some of them $(>30 \%)$ at full efficiency. (see Figure 78). Detailed studies revealed large regions of

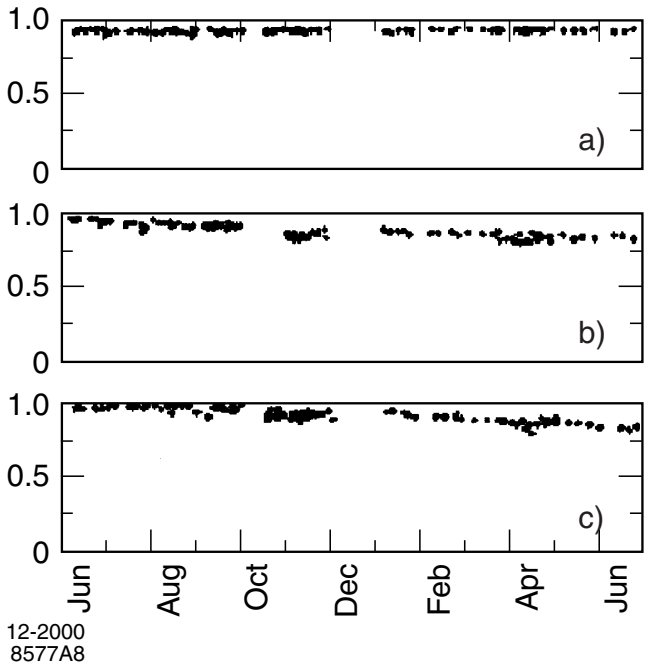

Figure 78. Efficiency history for 12 months starting in June 1999 for RPC modules showing different performance: a) highly efficient and stable; b) continuous slow decrease in efficiency; c) more recent, faster decrease in efficiency.

very low efficiency in these modules, but no clear pattern was identified.

The cause of the efficiency loss remains under investigation. Several possible causes have been excluded as the primary source of the problem, such as a change in the bakelite bulk resistivity, loosened spacers, gas flow, or gas composition. A number of prototype RPCs developed similar efficiency problems after being operated above a temperature $36^{\circ} \mathrm{C}$ for a period of two weeks. In some of these modules, evidence was found that the linseed oil had failed to cure and had accumulated at various spots under the influence of the electric field.

\subsection{Muon Identification}

While muon identification relies almost entirely on the IFR, other detector systems provide complementary information. Charged particles are reconstructed in the SVT and DCH and muon candidates are required to meet the criteria for minimum ionizing particles in the EMC. Charged tracks that are reconstructed in the tracking systems are extrapolated to the IFR taking into account the non-uniform magnetic field, multiple 
scattering, and the average energy loss. The projected intersections with the RPC planes are computed and for each readout plane all clusters detected within a predefined distance from the predicted intersection are associated with the track.

A number of variables are defined for each IFR cluster associated with a charged track to discriminate muons from charged hadrons: 1) the total number of interaction lengths traversed from the IP to the last RPC layer with an associated cluster, 2) the difference between this measured number of interaction lengths and the number of interaction lengths predicted for a muon of the same momentum and angle, 3) the average number and the rms of the distribution of RPC strips per layer, 4) the $\chi^{2}$ for the geometric match between the projected track and the centroids of clusters in different RPC layers, and 5) the $\chi^{2}$ of a polynomial fit to the two-dimensional IFR clusters. Selection criteria based on these variables are applied to identify muons.

The performance of muon identification has been tested on samples of muons from $\mu \mu e e$ and $\mu \mu \gamma$ final states and pions from three-prong $\tau$ decays and $K_{S} \rightarrow \pi^{+} \pi^{-}$decays. The selection of these control samples is based on kinematic variables, and not on variables used for muon identification. As illustrated in Figure 79, a muon detection efficiency of close to $90 \%$ has been achieved in the momentum range of $1.5<p<3.0 \mathrm{GeV} / c$ with a fake rate for pions of about $6-8 \%$. Decays in flight contribute about $2 \%$ to the pion misidentification probability. The hadron misidentification can be reduced by a factor of about two by tighter selection criteria which lower the muon detection efficiency to about $80 \%$.

\section{9. $K_{L}^{0}$ and Neutral Hadron Detection}

$K_{L}^{0}$ 's and other neutral hadrons interact in the steel of the IFR and can be identified as clusters that are not associated with a charged track. Monte Carlo simulations predict that about $64 \%$ of $K_{L}$ 's above a momentum of $1 \mathrm{GeV} / c$ produce a cluster in the cylindrical RPC, or a cluster with hits in two or more planar RPC layers.

Unassociated clusters that have an angular separation of $\leq 0.3 \mathrm{rad}$ are combined into a composite cluster, joining clusters that originate from
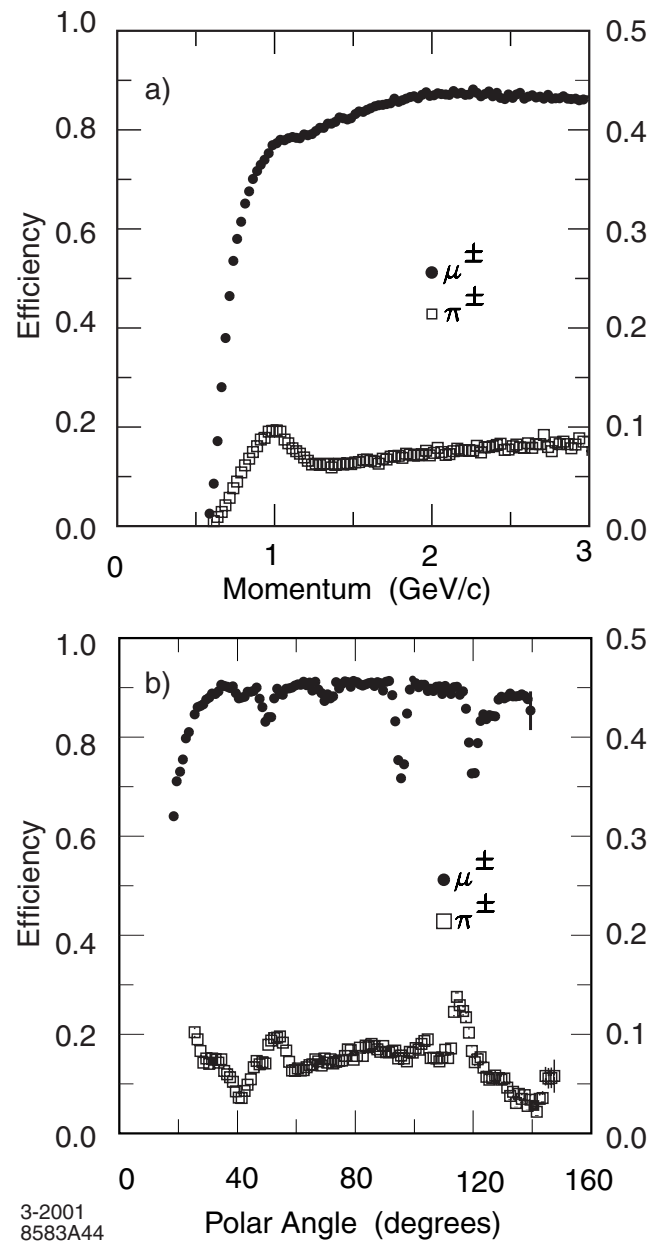

Figure 79. Muon efficiency (left scale) and pion misidentification probability (right scale) as a function of a) the laboratory track momentum, and $\mathrm{b}$ ) the polar angle (for $1.5<p<3.0 \mathrm{GeV} / c$ momentum), obtained with loose selection criteria.

showers that spread into adjacent sectors of the barrel, several sections of the end doors and/or the cylindrical RPC. This procedure also combines multiple clusters from large fluctuations in the hadronic showers. The direction of the neutral hadron is determined from the event vertex and the centroid of the neutral cluster. No information on the energy of the cluster can be obtained. 


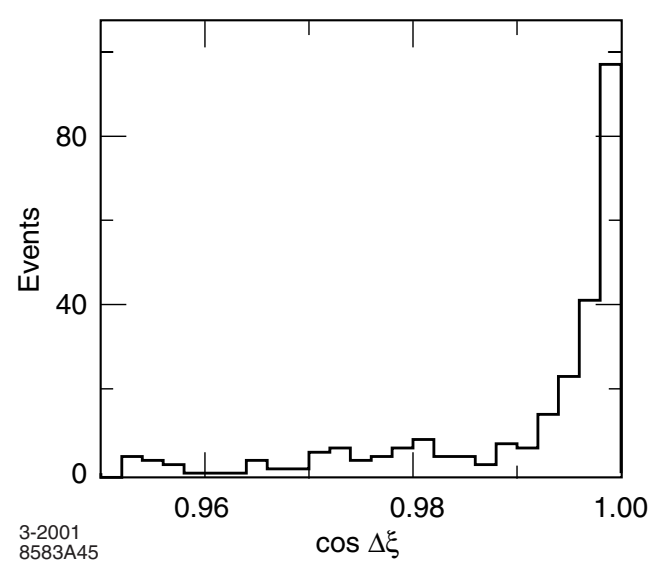

Figure 80. Angular difference, $\cos \Delta \xi$, between the direction of the missing momentum and the closest neutral IFR cluster for a sample of $\phi$ mesons produced in the reaction $e^{+} e^{-} \rightarrow \phi \gamma$ with $\phi \rightarrow K_{L}^{0} K_{S}^{0}$.

Since a significant fraction of hadrons interact before reaching the IFR, information from the EMC and the cylindrical RPCs is combined with the IFR cluster information. Neutral showers in the EMC are associated with the neutral hadrons detected in the IFR, based on a match in production angles. For a good match, a $\chi^{2}$ probability of $\geq 1 \%$ is required.

An estimate of the angular resolution of the neutral hadron cluster can be derived from a sample of $K_{L}^{0}$ 's produced in the reaction $e^{+} e^{-} \rightarrow$ $\phi \gamma \rightarrow K_{L}^{0} K_{S}^{0} \gamma$. The $K_{L}^{0}$ direction is inferred from the missing momentum computed from the measured particles in the final state. The data in Figure 80 indicate that the angular resolution of the $K_{L}^{0}$ derived from the IFR cluster information is of the order of $60 \mathrm{mrad}$. For $K_{L}^{0}$ 's interacting in the EMC, the resolution is better by about a factor of two.

For multi-hadron events with a reconstructed $J / \psi$ decay, Figure 81 shows the angular difference, $\Delta \phi$, between the missing momentum and the direction of the nearest neutral hadron cluster. The observed peak demonstrates clearly that the missing momentum can be associated with a neutral hadron, assumed to be a $K_{L}^{0}$. The $K_{L}^{0}$ detection efficiency increases roughly linearly with momentum; it varies between $20 \%$ and $40 \%$ in the

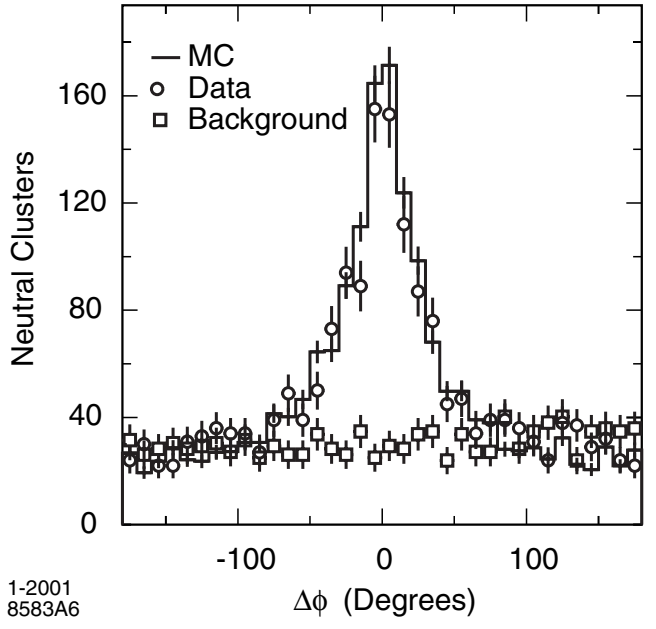

Figure 81. Difference between the direction of the reconstructed neutral hadron cluster and the missing transverse momentum in events with a reconstructed $J / \psi$ decay. The Monte Carlo simulation is normalized to the luminosity of the data; the background is obtained using neutral hadrons and the missing momentum from different events.

momentum range from $1 \mathrm{GeV} / c$ to $4 \mathrm{GeV} / c$ (EMC and IFR combined).

\subsection{Summary and Outlook}

The IFR is the largest RPC system built to date. It provides efficient muon identification and allows for the detection of $K_{L}^{0}$ 's interacting in the steel and the calorimeter. During the first year of operation, a large fraction of the RPC modules have suffered significant losses in efficiency. This effect appears to be correlated with high temperatures, but the full extent of the problem and its cause remain under study. Thanks to the large number of RPC layers, this problem has not yet impacted the overall performance severely. But present extrapolations, even after installation of water cooling on the steel, indicate a severe problem for the future operation. Recently, 24 end door modules have been replaced by new RPCs with a substantially thinner coating of linseed oil and improved treatment of the bakelite surfaces. Results with these new RPCs and other tests will need to be evaluated before decisions on future improvements of the IFR can be made. Further- 
more, it is planned to reduce the contamination from hadron decays and punch through by increasing the absorber thickness, i.e., adding more steel on the outside and replacing a few of the RPCs with absorber plates.

\section{Trigger}

\subsection{Trigger Requirements}

The basic requirement for the trigger system is the selection of events of interest (see Table 14) with a high, stable, and well-understood efficiency while rejecting background events and keeping the total event rate under $120 \mathrm{~Hz}$. At design luminosity, beam-induced background rates are typically about $20 \mathrm{kHz}$ each for one or more tracks in the drift chamber with $p_{\mathrm{t}}>120 \mathrm{MeV} / c$ or at least one EMC cluster with $E>100 \mathrm{MeV}$. Efficiency, diagnostic, and background studies require prescaled samples of special event types, such as those failing the trigger selection criteria, and random beam crossings.

The total trigger efficiency is required to exceed $99 \%$ for all $B \bar{B}$ events and at least $95 \%$ for continuum events. Less stringent requirements apply to other event types, e.g., $\tau^{+} \tau^{-}$events should have a 90-95\% trigger efficiency, depending on the specific $\tau^{ \pm}$decay channels.

The trigger system must be robust and flexible in order to function even under extreme background situations. It must also be able to operate in an environment with dead or noisy electronics channels. The trigger should contribute no more than $1 \%$ to dead time.

\subsection{Trigger Overview}

The trigger is implemented as a two-level hierarchy, the Level 1 (L1) in hardware followed by the Level 3 (L3) in software. It is designed to accommodate up to ten times the initially projected [3] PEP-II background rates at design luminosity and to degrade slowly for backgrounds above that level. Redundancy is built into the system to measure and monitor trigger efficiencies.

During normal operation, the L1 is configured to have an output rate of typically $1 \mathrm{kHz}$. Triggers are produced within a fixed latency window of 11$12 \mu$ s after the $e^{+} e^{-}$collision, and delivered to the
Fast Control and Timing System (FCTS). Data used to form the trigger decision are preserved with each event for efficiency studies.

The $\mathrm{L} 3$ receives the output from $\mathrm{L} 1$, performs a second stage rate reduction for the main physics sources, and identifies and flags the special categories of events needed for luminosity determination, diagnostic, and calibration purposes. At design luminosity, the L3 filter acceptance for physics is $\sim 90 \mathrm{~Hz}$, while $\sim 30 \mathrm{~Hz}$ contain the other special event categories. The L3 algorithms comply with the same software conventions and standards used in all other BABAR software, thereby simplifying its design, testing, and maintenance.

\subsection{Level 1 Trigger System}

The L1 trigger decision is based on charged tracks in the DCH above a preset transverse momentum, showers in the EMC, and tracks detected in the IFR. Trigger data are processed by three specialized hardware processors. As described below, the drift chamber trigger (DCT) and electromagnetic calorimeter trigger (EMT) both satisfy all trigger requirements independently with high efficiency, and thereby provide a high degree of redundancy, which enables the measurement of trigger efficiency. The instrumented flux return trigger (IFT) is used for triggering $\mu^{+} \mu^{-}$and cosmic rays, mostly for diagnostic purposes.

The overall structure of the L1 trigger is illus-

Table 14

Cross sections, production and trigger rates for the principal physics processes at $10.58 \mathrm{GeV}$ for a luminosity of $3 \times 10^{33} \mathrm{~cm}^{-2} \mathrm{~s}^{-1}$. The $e^{+} e^{-}$cross section refers to events with either the $e^{+}, e^{-}$, or both inside the EMC detection volume.

\begin{tabular}{lccc}
\hline $\begin{array}{l}\text { Event } \\
\text { type }\end{array}$ & $\begin{array}{c}\text { Cross } \\
\text { section } \\
(\mathrm{nb})\end{array}$ & $\begin{array}{c}\text { Production } \\
\text { Rate } \\
(\mathrm{Hz})\end{array}$ & $\begin{array}{c}\text { Level 1 } \\
\text { Trigger } \\
\text { Rate }(\mathrm{Hz})\end{array}$ \\
\hline$b \bar{b}$ & 1.1 & 3.2 & 3.2 \\
other $q \bar{q}$ & 3.4 & 10.2 & 10.1 \\
$e^{+} e^{-}$ & $\sim 53$ & 159 & 156 \\
$\mu^{+} \mu^{-}$ & 1.2 & 3.5 & 3.1 \\
$\tau^{+} \tau^{-}$ & 0.9 & 2.8 & 2.4 \\
\hline
\end{tabular}




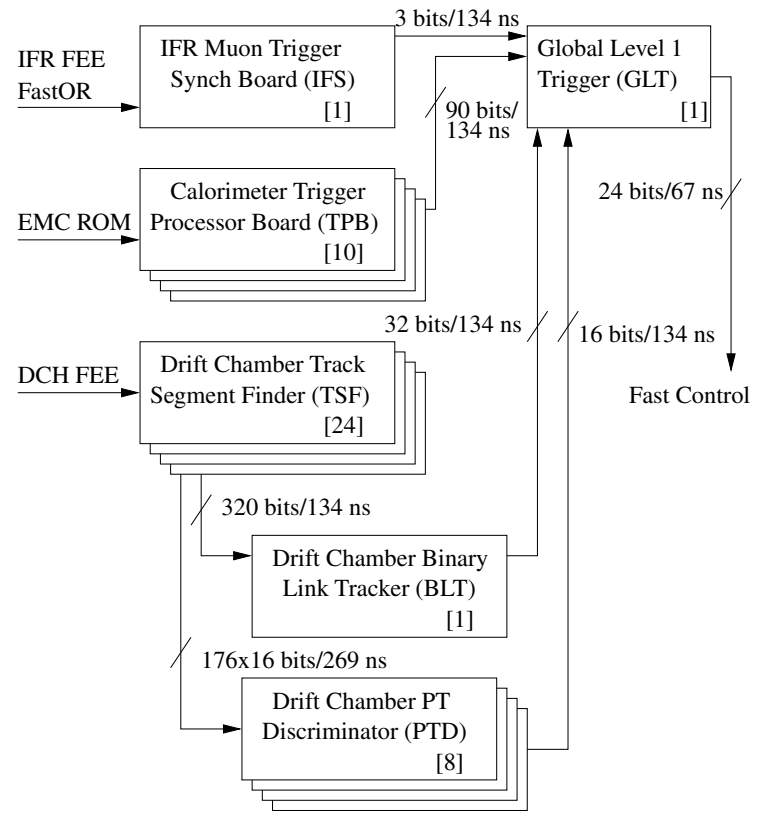

Figure 82. Simplified L1 trigger schematic. Indicated on the figure are the number of components (in square brackets), and the transmission rates between components in terms of total signal bits.

trated in Figure 82. Each of the three L1 trigger processors generates trigger primitives, summary data on the position and energy of particles, that are sent to the global trigger (GLT) every $134 \mathrm{~ns}$. The DCT and EMT primitives sent to the GLT are $\phi$-maps. An individual $\phi$-map consists of an $n$-bit word representing a particular pattern of trigger objects as distributed in fixed-width $\phi$ regions from 0 to $2 \pi$. A trigger object is a quantity indicating the presence of a particle, such as a drift chamber track or a calorimeter energy deposit. The IFT primitive is a three-bit pattern representing the hit topology in the IFR. The meaning of the various trigger primitive inputs to the GLT are summarized in Table 15.

The GLT processes all trigger primitives to form specific triggers and then delivers them to the FCTS. The FCTS can optionally mask or prescale any of these triggers. If a valid trigger remains, a L1 Accept is issued to initiate event readout. The trigger definition logic, masks, and prescale values are all configurable on a per run basis.

The L1 hardware is housed in five 9U VME crates. The L1 trigger operates in a continuous sampling mode, generating trigger information at regular, fixed time intervals. The $\mathrm{DCH}$ front-end electronics (FEEs) and the EMC untriggered personality cards (UPCs) send raw data to the DCT and EMT about $2 \mu$ s after the $e^{+} e^{-}$collision. The DCT and EMT event processing times are $4-5 \mu \mathrm{s}$, followed by another $\sim 3 \mu \mathrm{s}$ of processing in the GLT to issue a L1 trigger. The L1 trigger takes approximately $1 \mu \mathrm{s}$ to propagate through the FCTS and the readout modules (ROMs) to initiate event readout. These steps are all accomplished within the $12.8 \mu$ s FEE buffer capacity limit.

The DCT, EMT and GLT each maintain a fourevent buffer to hold information resulting from the various stages of the L1 trigger. These data are read out by the normal data acquisition system.

\subsubsection{Level 1 Drift Chamber Trigger}

The input data to the DCT consist of one bit for each of the 7104 DCH cells. These bits convey time information derived from the sense wire signal for that cell. The DCT output primitives are candidate tracks encoded in terms of three 16-bit $\phi$-maps as listed in Table 15.

The DCT algorithms are executed in three types of modules [106]. First, track segments, their $\phi$ positions and drift time estimates are found using a set of 24 Track Segment Finder (TSF) modules [107]. These data are then passed to the Binary Link Tracker (BLT) module [108], where segments are linked into complete tracks. In parallel, the $\phi$ information for segments found in axial superlayers is transmitted to eight transverse momentum discriminator (PTD) modules [109], which search for tracks above a set $p_{\mathrm{t}}$ threshold.

Each of the three DCT modules (TSF, BLT, and PTD) relies heavily on multiple FPGA's [110] which perform the control and algorithmic functions. All cabling is handled by a small $(6 \mathrm{U})$ backof-crate interface behind each main board. 
Table 15

Trigger primitives for the DCT and EMT. Most energy thresholds are adjustable; those listed are typical values.

\begin{tabular}{lllcc}
\hline \hline & Description & Origin & No. of bits & Threshold \\
\hline B & Short track reaching DCH superlayer 5 & BLT & 16 & $120 \mathrm{MeV} / c$ \\
A & Long track reaching DCH superlayer 10 & BLT & 16 & $180 \mathrm{MeV} / c$ \\
A $^{\prime}$ & High $p_{\text {t }}$ track & PTD & 16 & $800 \mathrm{MeV} / c$ \\
\hline M & All- $\theta$ MIP energy $\theta$ intermediate energy & TPB & 20 & $100 \mathrm{MeV}$ \\
G & All $\theta$ TPB & 20 & $250 \mathrm{MeV}$ \\
E & All- $\theta$ high energy & TPB & 20 & $700 \mathrm{MeV}$ \\
X & Forward endcap MIP & TPB & 20 & $100 \mathrm{MeV}$ \\
Y & Backward barrel high energy & TPB & 10 & $1 \mathrm{GeV}$ \\
\hline \hline
\end{tabular}

\section{Track Segment Finder}

The TSF modules are responsible for finding track segments in 1776 overlapping eight-cell pivot groups. A pivot group is a contiguous set of cells that span all four layers within a superlayer. The pivot group shape is such that only reasonably straight tracks originating from the interaction point can produce a valid segment. Figure 83 shows the arrangement of cells within a pivot group. Cell 4 is called the pivot cell; the TSF algorithm is optimized to find track segments that pivot about this cell.

The DCH signals are sampled every $269 \mathrm{~ns}$. The passage of a single particle through the DCH will produce ionization that drifts to the sense wires in typically no more than four of these clock ticks. Each cell is associated with a two-bit counter that is incremented at every clock tick for which a signal is present. In this way, a short time history of each cell is preserved. For each clock tick, the collection of two-bit counters for each pivot group forms a 16-bit value used to address a look-up-table. This look-up-table contains twobit weights indicating whether there is no acceptable segment, a low-quality segment, a three-layer segment (allowing for cell inefficiencies), or a fourlayer segment. When an acceptable segment is found, that pivot group is examined to determine which of three subsequent clock ticks produce the highest weight or best pattern.

The look-up-table also contains position and time information which, along with a summary of

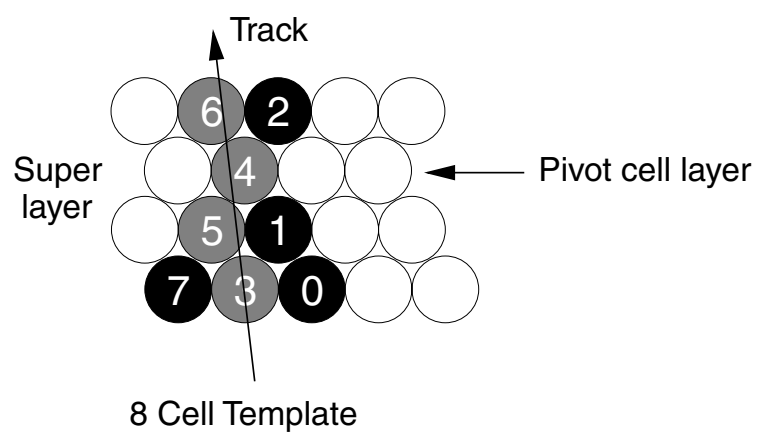

Figure 83. Track Segment Finder pivot group.

cell occupancies, forms the basis of data sent to the BLT and PTD. The TSF algorithm uses the time-variation of the look-up-table weights to refine both the event time and its uncertainty, thus enabling it to output results to the BLT every $134 \mathrm{~ns}$.

The position resolution as measured from the data after calibration, is typically $\sim 600 \mu \mathrm{m}$ for a four-layer segment and $\sim 900 \mu \mathrm{m}$ for a three-layer segment. For tracks originating from the IP, the efficiency for finding TSF segments is $97 \%$, and the efficiency for high-quality three-layer or fourlayer TSF segments is $94 \%$.

\section{Binary Link Tracker}

The BLT receives segment hit information from all 24 TSF's at a rate of 320 bits every $134 \mathrm{~ns}$ and links them into complete tracks. The segment hits are mapped onto the DCH geometry in 
terms of 320 supercells, 32 sectors in $\phi$ and ten radial superlayers. Each bit indicates whether a segment is found in that supercell or not. The BLT input data are combined using a logical OR with a programmable mask pattern. The masking allows the system to activate track segments corresponding to dead or highly inefficient cells to prevent efficiency degradation. The linking algorithm uses an extension of a method developed for the CLEO-II trigger [111]. It starts from the innermost superlayer, A1, and moves radially outward.

Tracks that reach the outer layer of the $\mathrm{DCH}$ (superlayer A10) are classified as type A. Tracks that reach the middle layer (superlayer U5) are classified as type B. An A track is found if there is a segment in at least eight superlayers and if the segments in two consecutive superlayers fall azimuthally within three to five supercells of each other (depending on the superlayer type). This allows for track curvature and dip angle variations. The data are compressed and output to the GLT in the form of two 16-bit $\phi$-maps, one each for A and B tracks.

\section{PT Discriminator}

The eight PTD modules receive $\phi$ information of high quality track segments in the axial superlayers (A1, A4, A7 and A10), and determine if the segments are consistent with a track $p_{\mathrm{t}}$ greater than a configurable minimum value. An envelope for tracks above the minimum $p_{\mathrm{t}}$ is defined using the IP, and a track segment position in one of the seed superlayers, A7 or A10. A high $p_{\mathrm{t}}$ candidate, denoted as $\mathrm{A}^{\prime}$, is identified when sufficient track segments with accurate $\phi$ information from the other axial superlayers lie within this envelope.

Each PTD module searches for seed segments in superlayers A7 and A10, and within a 45degree azimuthal wedge of the DCH. This search region spans eight supercells, and the processing for each supercell is performed by its own processing engine on the PTD. The principal components in each engine are an algorithmic processor and look-up-tables containing the limits for each individual seed position. The contents of the lookup-tables specify the allowed track segment positions for each of the three other axial superlayers

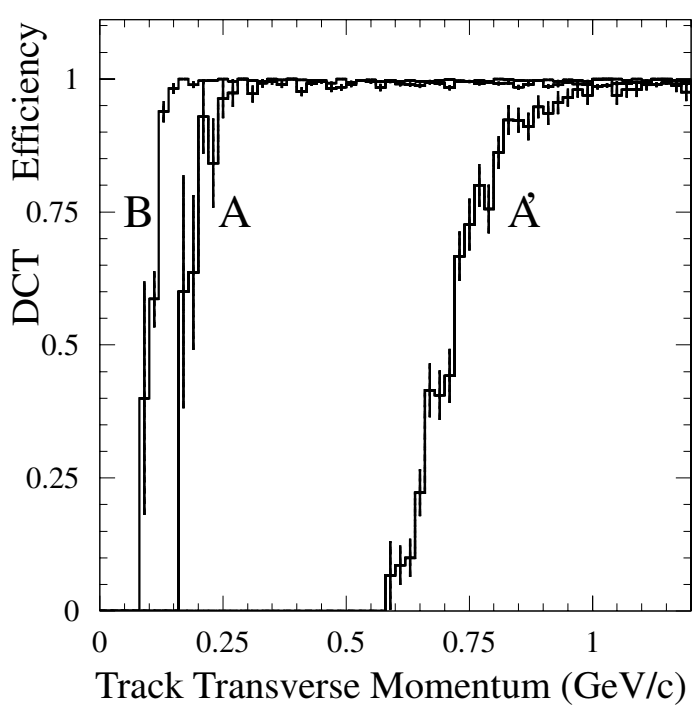

Figure 84. DCT track efficiency versus transverse momentum for $\mathrm{A}, \mathrm{B}$, and $\mathrm{A}^{\prime}$ tracks. The $\mathrm{A}^{\prime}$ threshold is set to $800 \mathrm{MeV} / c$.

and consequently define the effective $p_{\mathrm{t}}$ discrimination threshold. The resulting $p_{\mathrm{t}}$ threshold for the PTD A' tracks is shown in Figure 84 together with the BLT A, B track efficiency.

\subsubsection{Level 1 Calorimeter Trigger}

For trigger purposes, the EMC is divided into 280 towers, $7 \times 40(\theta \times \phi)$. Each of the barrel's 240 towers is composed of 24 crystals in a $8 \times$ $3(\theta \times \phi)$ array. The endcap is divided into 40 towers, each forming a wedge in $\phi$ containing 1922 crystals. For each tower, all crystal energies above a threshold of $20 \mathrm{MeV}$ are summed and sent to the EMT every $269 \mathrm{~ns}$.

The conversion of the tower data into the GLT $\phi$-maps is performed by ten Trigger Processor Boards (TPBs). The TPBs determine energies in the $40 \phi$ sectors, summing over various ranges of $\theta$, compare these energies against thresholds for each of the trigger primitives (see Table 15), estimate the time of energy deposition, correct for timing jitter, and then transmit the result to the GLT.

Each TPB receives data from 28 towers, corresponding to an array of $7 \times 4$ in $\theta \times \phi$, or four $\phi$ sectors. Each of the $40 \phi$-sectors is summed independently. To identify energy deposits that span 
two adjacent $\phi$-sectors, the energy of each sector is also made available to the summing circuit for a single adjoining sector in such a way that all possible pairs of adjacent $\phi$-sectors are summed. These energy sums are compared against thresholds to form trigger objects. Each sum is also sent to an eight-tap finite impulse response (FIR) digital filter which is used to estimate the energy deposition time. A look-up-table is used to make an energy-dependent estimate of the timing jitter which, along with the FIR output, is used to time the transmission of any trigger objects to the GLT. Pairs of $\phi$-sectors are ORed to form 20-bit $\phi$-maps for the M, G, E, and X primitives, while for the Y primitive, groups of four are ORed to form a 10-bit $\phi$-map. The complete algorithm is implemented in one FPGA [112] for each $\phi$-sector, with four identical components per TPB. Further details of the EMT system can be found in [113].

The basic performance of the EMT can be expressed in terms of the efficiency and timing jitter of the trigger primitives. The efficiency of the primitives can be measured by the number of times a trigger bit is set for a specific energy reconstructed offline in events from a random trigger. Figure 85 shows this efficiency for energies near the $\mathrm{M}$ threshold. The efficiency changes from $10 \%$ to $90 \%$ in the range of 110 to $145 \mathrm{MeV}$, and reaches $99 \%$ at $180 \mathrm{MeV}$, close to the average energy deposition of a minimum ionizing particle at normal incidence.

The EMT time jitter is measured by comparing the time centroid of $\phi$-strip M hits in $\mu^{+} \mu^{-}$events with the DCH track start time, $t_{0}$. The difference has an rms width of $90 \mathrm{~ns}$ with $>99.9 \%$ of the matching $M$ hits within a \pm 500 ns window.

\subsubsection{Level 1 IFR Trigger}

The IFT is used for triggering on $\mu^{+} \mu^{-}$and cosmic rays. For the purposes of the trigger, the IFR is divided into ten sectors, namely the six barrel sextants and the four half end doors. The inputs to the IFT are the Fast OR signals of all $\phi$ readout strips in eight selected layers in each sector.

A majority logic algorithm defines trigger objects for every sector in which at least four of the eight trigger layers have hits within a time win-

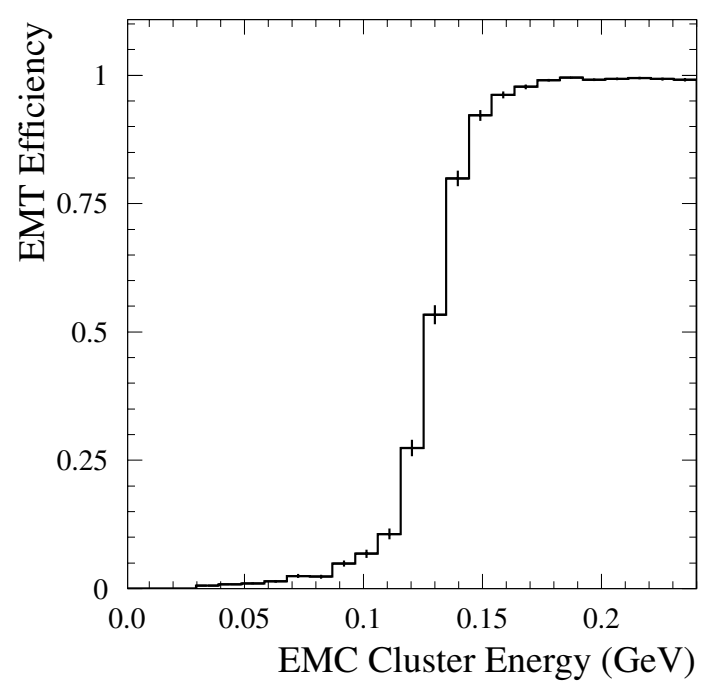

Figure 85. EMT M efficiency vs. EMC cluster energy for an M threshold setting of $120 \mathrm{MeV}$.

Table 16

IFR trigger pattern (U) definition, where $\mu$ refers to a signal within a sector.

\begin{tabular}{ll}
\hline $\mathrm{U}$ & Trigger condition \\
\hline 1 & $\geq 2 \mu$ topologies other than $\mathrm{U}=5-7$ \\
2 & $1 \mu$ in backward endcap \\
3 & $1 \mu$ in forward endcap \\
4 & $1 \mu$ in barrel \\
5 & 2 back-back $\mu$ 's in barrel +1 forward $\mu$ \\
6 & $1 \mu$ in barrel +1 forward $\mu$ \\
7 & 2 back-back $\mu$ 's in barrel \\
\hline
\end{tabular}

dow of $134 \mathrm{~ns}$. The IFR trigger synchronization module processes the trigger objects from the ten sectors and generates the three-bit trigger word (U) encoding seven exclusive trigger conditions, as defined in Table 16. The trigger $\mathrm{U} \geq 5$, for example, covers all $\mu^{+} \mu^{-}$topologies of interest.

The efficiency of the IFT has been evaluated using cosmic rays triggered by the DCT and crossing the detector close to the IP. For these events, $98 \%$ were triggered by the IFT as events with at least one track, and $73 \%$ as events with two tracks, inside the geometrical region of the IFR. 
Most of the IFT inefficiency is concentrated at the boundaries between sectors.

\subsubsection{Global Trigger}

The GLT receives the eight trigger primitives in the form of $\phi$-maps as listed in Table 15 along with information from the IFT (Table 16) to form specific triggers that are then passed to the FCTS for the final trigger decision. Due to the different latencies associated with the production of these primitives, the GLT forms a time alignment of these input data using configurable delays.

The GLT then forms some additional combined $\phi$-maps from the DCT and EMT data. These maps include matched objects such as BM for B tracks matched to an $\mathrm{M}$ cluster in $\phi$, back-to-back objects, $\mathrm{B}^{*}$ and $\mathrm{M}^{*}$, which require a pair of $\phi$ bits separated by a configurable angle of typically $\sim 120^{\circ}$, and an EM* object for back-to-back EM pairs.

All $16 \phi$-maps are then used to address individual GLT look-up-tables which return three-bit counts of trigger objects contained within those maps, e.g., the number of B tracks or number of $\mathrm{M}$ clusters. To count as distinct trigger objects, the map bits are typically required to have a separation of more than one $\phi$ bin. The resulting 16 counts plus the IFT hit pattern are then tested in logical operations. The permissible operations include: always-pass; or a comparison $(\geq$, $=$, or $<$ ) with a configurable selection parameter. A trigger line is then set as the logical AND of these 17 operations. This process is performed for each of the 24 trigger lines.

The GLT derives the L1 trigger time from the centroid of the timing distribution of the highest priority trigger, binned in the $134 \mathrm{~ns}$ interval and spanning about $1 \mu \mathrm{s}$. Other trigger signals compatible with this time are retained and cached. The average time is calculated to the nearest $67 \mathrm{~ns}$ and the 24-bit GLT output signal is sent to the FCTS every $67 \mathrm{~ns}$. The achieved timing resolution for hadronic events has an rms width of $52 \mathrm{~ns}$; and $99 \%$ of the events are within $77 \mathrm{~ns}$.

The GLT hardware consists of a single $9 \mathrm{U}$ VME module. Most of the logic, including diagnostic and DAQ memories, are implemented in FPGA's [110]. The look-up-table section is im- plemented as an array of 16 memory chips with 8 Mbytes of configuration data.

\subsection{Level 1 Trigger Performance and Operational Experience}

The L1 trigger configuration consists of DCTonly, EMT-only, mixed and prescaled triggers, aimed not only for maximum efficiency and background suppression, but also for the convenience of trigger efficiency determination.

Although most triggers target a specific physics source, they often also select other processes. For example, two-track triggers are not only efficient for Bhabha, $\mu^{+} \mu^{-}$, and $\tau^{+} \tau^{-}$events, but are also useful for selecting jet-like hadronic events and some rare $B$ decays.

The efficiencies and rates of selected L1 triggers for various physics processes are listed in Table 17. Although triggering on generic $B \bar{B}$ events is relatively easy, it is essential to ensure high efficiencies for the important rare lowmultiplicity $B$ decays. For this reason, efficiencies for $B^{0} \rightarrow \pi^{0} \pi^{0}$ and $B^{-} \rightarrow \tau^{-} \bar{\nu}$ are also listed in Table 17 .

The efficiencies listed for the hadronic events are absolute and include acceptance losses based on Monte Carlo simulation, and local inefficiency effects. The efficiencies for $\tau$-pair events are for fiducial events, i.e., events with two or more tracks with $p_{\mathrm{t}}>120 \mathrm{MeV} / c$ and polar angle $\theta$ to reach at least DCH superlayer U5. The Bhabha and $\mu$-pair efficiencies are determined from the data, for events with two high momentum particles, which are back-to-back in the c.m. system, and within the EMC fiducial volume. The data in Table 17 demonstrate that the DCT and the combined EMT/IFT provide fully efficient, independent triggers for most physics processes, although independent triggers for $\mu^{+} \mu^{-}$and $\tau^{+} \tau^{-}$are not individually fully efficient. The efficiencies predicted by the Monte Carlo simulation are generally in good agreement with data when tested using events passing typical analysis selections and based on orthogonal triggers. Prescaled triggers with a very open acceptance of physics events, such as $(B \geq 2 \& A \geq 1)$ or $(M \geq 2)$ are also used to measure the trigger efficiencies.

The trigger rates listed in Table 17 are for a 
Table 17

Level 1 Trigger efficiencies (\%) and rates (Hz) at a luminosity of $2.2 \times 10^{33} \mathrm{~cm}^{-2} \mathrm{~s}^{-1}$ for selected triggers applied to various physics processes. The symbols refer to the counts for each object.

\begin{tabular}{|c|c|c|c|c|c|c|c|c|c|}
\hline Level 1 Trigger & $\varepsilon_{B \bar{B}}$ & $\varepsilon_{B \rightarrow \pi^{0} \pi^{0}}$ & $\varepsilon_{B \rightarrow \tau \bar{\nu}}$ & $\varepsilon_{c \bar{c}}$ & $\varepsilon_{u d s}$ & $\varepsilon_{e e}$ & $\varepsilon_{\mu \mu}$ & $\varepsilon_{\tau \tau}$ & Rate \\
\hline $\mathrm{A} \geq 3 \quad \& \mathrm{~B}^{*} \geq 1$ & 97.1 & 66.4 & 81.8 & 88.9 & 81.1 & - & - & 17.7 & 180 \\
\hline $\mathrm{A} \geq 1 \& \mathrm{~B}^{*} \geq 1 \& \mathrm{~A}^{\prime} \geq 1$ & 95.0 & 63.0 & 83.2 & 89.2 & 85.2 & 98.6 & 99.1 & 79.9 & 410 \\
\hline Combined DCT (ORed) & 99.1 & 79.7 & 92.2 & 95.3 & 90.6 & 98.9 & 99.1 & 80.6 & 560 \\
\hline $\mathrm{M} \geq 3 \quad \& \mathrm{M}^{*} \geq 1$ & 99.7 & 98.6 & 93.7 & 98.5 & 94.7 & - & 一 & 53.7 & 160 \\
\hline $\mathrm{EM}^{*} \geq 1$ & 71.4 & 94.9 & 55.5 & 77.1 & 79.5 & 97.8 & - & 65.8 & 150 \\
\hline Combined EMT (ORed) & 99.8 & 99.2 & 95.5 & 98.8 & 95.6 & 99.2 & - & 77.6 & 340 \\
\hline $\mathrm{B} \geq 3 \quad \& \quad \mathrm{~A} \geq 2 \quad \& \quad \mathrm{M} \geq 2$ & 99.4 & 81.2 & 90.3 & 94.8 & 87.8 & - & - & 19.7 & 170 \\
\hline $\mathrm{M}^{*} \geq 1 \& \mathrm{~A} \geq 1 \& \mathrm{~A}^{\prime} \geq 1$ & 95.1 & 68.8 & 83.7 & 90.1 & 87.0 & 97.8 & 95.9 & 78.2 & 250 \\
\hline $\mathrm{E} \geq 1 \quad \& \quad \mathrm{~B} \geq 2 \quad \& \quad \mathrm{~A} \geq 1$ & 72.1 & 92.4 & 60.2 & 77.7 & 79.2 & 99.3 & - & 72.8 & 140 \\
\hline $\mathrm{M}^{*} \geq 1 \& \mathrm{U} \geq 5$ ( $\mu$-pair $)$ & - & - & - & - & - & - & 60.3 & - & 70 \\
\hline Combined Level 1 triggers & $>99.9$ & 99.8 & 99.7 & 99.9 & 98.2 & $>99.9$ & 99.6 & 94.5 & 970 \\
\hline
\end{tabular}

typical run with HER (LER) currents at $650 \mathrm{~mA}$ $(1350 \mathrm{~mA})$ and a luminosity of $2.2 \times 10^{33} \mathrm{~cm}^{-2} \mathrm{~s}^{-1}$. These rates are stable to within $20 \%$ for the same PEP-II configuration, but they are impacted by changes in vacuum conditions, beam currents, and orbits. There are occasional background spikes which can double the L1 rate. However, due to the $2 \mathrm{kHz}$ capability of the data acquisition, these spikes do not induce significant dead time.

For a typical L1 rate of $1 \mathrm{kHz}$, Bhabha and annihilation physics events contribute $\sim 130 \mathrm{~Hz}$. There are also $100 \mathrm{~Hz}$ of cosmic ray and $20 \mathrm{~Hz}$ of random beam crossing triggers. The remaining triggers are due to lost particles interacting with the beam pipe or other components. The distribution of single track $z_{0}$ values as reconstructed by L3 for all L1 triggers is shown in Figure 86. The most prominent peaks at $z= \pm 20 \mathrm{~cm}$ correspond to a flange of the beam pipe. The peak at $z_{0}=-55 \mathrm{~cm}$ corresponds to a step in the synchrotron mask.

The L1 trigger hardware operation has been very stable. For the first one and half years of operation, there have been only four hardware failures in the L1 system, mainly auxiliary or communication boards. Occasional adjustments to the EMT tower mask were used to temporarily

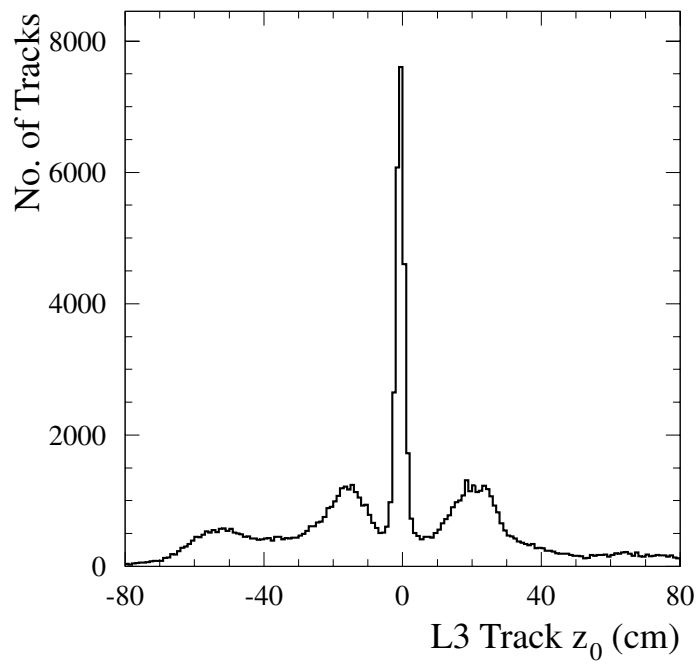

Figure 86. Single track $z_{0}$ for all L1 tracks, reconstructed by L3.

suppress noisy channels in the EMC electronics.

\subsection{Level 3 Trigger System}

The L3 trigger software comprises event reconstruction and classification, a set of event selection filters, and monitoring. This software runs on the online computer farm. The filters have access to the complete event data for making their decision, including the output of the L1 trigger 
processors and FCTS trigger scalers. L3 operates by refining and augmenting the selection methods used in L1. For example, better DCH tracking (vertex resolution) and EMC clustering filters allow for greater rejection of beam backgrounds and Bhabha events.

The L3 system runs within the Online Event Processing (OEP) framework (see Section 12). OEP delivers events to L3, then prescales and logs those which pass the L3 selection criteria.

To provide optimum flexibility under different running conditions, L3 is designed according to a general logic model that can be configured to support an unlimited variety of event selection mechanisms. This provides for a number of different, independent classification tests, called scripts, that are executed independently, together with a mechanism for combining these tests into the final set of classification decisions.

The L3 trigger has three phases. In the first phase, events are classified by defining L3 input lines, which are based on a logical OR of any number of the 32 FCTS output lines. Any number of L3 input lines may be defined.

The second phase comprises a number of scripts. Each script executes if its single L3 input line is true and subsequently produces a single pass-fail output flag. Internally, a script may execute one or both of the DCH or EMC algorithms, followed by one or more filters. The algorithms construct quantities of interest, while the filters determine whether or not those quantities satisfy the specific selection criteria.

In the final phase, the L3 output lines are formed. Each output line is defined as the logical OR of selected script flags. L3 can treat script flags as vetoes, thereby rejecting, for example, carefully selected Bhabha events which might otherwise satisfy the selection criteria.

L3 utilizes the standard event data analysis framework and depends crucially on several of its aspects. Any code in the form of modules can be included and configured at run time. A sequence of these software modules compose a script. The same instance of a module may be included in multiple scripts yet it is executed only once, thus avoiding significant additional CPU overhead.

\subsubsection{Level 3 Drift Chamber Tracking Algorithm}

Many events which pass L1 but must be rejected by L3 are beam-induced charged particle background that are produced in material close to the IP. L1 does not currently have sufficient tracking resolution to identify these background tracks. The DCH-based algorithm, L3Dch, performs fast pattern recognition (track finding) and track fitting, which determines the five helix track parameters for tracks with $p_{\mathrm{t}}$ above $250 \mathrm{MeV} / c$. To speed up the process of pattern recognition, L3Dch starts with the track segments from the TSF system and improves the resolution by making use of the actual DCH information.

For those TSF segments that have a simple solution to the left-right ambiguity, a track $t_{0}$ is determined. The $t_{0}$ values for each segment in an event are binned and the mean produced from the values in the most populated bin is used as the estimated event $t_{0}$. All events which pass L1 typically have enough segments to form a $t_{0}$ estimate. The measured rms resolution on this estimate is $1.8 \mathrm{~ns}$ for Bhabha events and 3.8 ns for hadronic events.

The pattern recognition for L3Dch is done with a look-up-table. For this track table, the DCH is divided into $120 \phi$-sectors, corresponding to the number of cells in the innermost layers. The track table is populated with the hit patterns of Monte Carlo generated tracks with a $p_{\mathrm{t}}$ above $250 \mathrm{MeV} / c$ and originating within $2 \mathrm{~cm}$ of the IP in the $x-y$ plane, and within $10 \mathrm{~cm}$ in $z$. The pattern recognition algorithm searches the table entries looking for matches to segments found by the TSFs. The matched set of segments for a given track is then passed to the track fitting algorithm. The track table allows for up to two missing DCH TSF segments per track.

The track fitting algorithm is provided with both the track segments found in pattern recognition and the individual hits within those segments. From this information the five helix parameters are fitted. The fit is then iterated, adding segments close to the initially fitted track, and dropping hits with large residuals. The final fit does not demand that the track originate from the IP. 


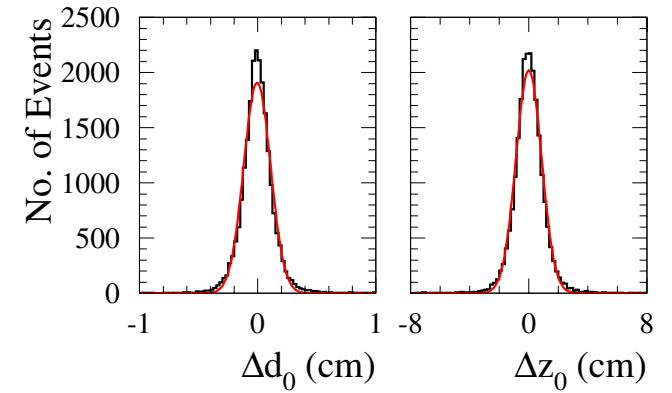

Figure 87. Transverse and longitudinal miss distances between the two tracks in Bhabha events.

The two-track miss distances for Bhabha events are plotted in Figure 87. The resolutions for individual tracks are $0.80 \mathrm{~mm}$ and $6.1 \mathrm{~mm}$ for $d_{0}$ and $z_{0}$, respectively. Similarly, the $1 / p_{\mathrm{t}}$ difference between the two tracks in $\mu$-pair events yields a $p_{\mathrm{t}}$ resolution of $\delta p_{\mathrm{t}} / p_{\mathrm{t}} \sim 0.019 \cdot p_{\mathrm{t}}$, with $p_{\mathrm{t}}$ in $\mathrm{GeV} / c$.

\subsubsection{Level 3 Calorimeter \\ Clustering Algorithm}

The all-neutral trigger for L3 is based on information from the EMC. In addition, calorimeter information is a vital complement to the DCH data for the identification of Bhabha events.

The L3 EMC-based trigger, L3Emc, identifies energy clusters with a sensitivity sufficient for finding minimum ionizing particles. EMC data are processed in two steps: first, lists of crystals with significant energy deposits are formed; and second, clusters are identified. The EMC typically sends data for $\sim 1400$ crystals (of 6580 total). The majority of these are caused by electronics noise and beam-induced background. For each crystal, these data include the peak energy and time of the crystal waveform. To filter out noise, L3Emc rejects individual crystal signals below an energy threshold of $20 \mathrm{MeV}$ or which lie outside a $1.3 \mu$ s time window around the event time. For the remaining crystals, raw energies and times are converted into physical units and added to the L3Emc crystal list. Clusters are formed using an optimized look-up-table technique requiring only a single pass over the crystal list. Clusters with a total energy above $100 \mathrm{MeV}$ are retained, and the energy weighted centroid and average time, the number of crystals, and a lateral moment describ- ing the shower shape for particle identification are calculated.

\subsubsection{Level 3 Filters}

Based on the L3 tracks and clusters, a variety of filters perform event classification and background reduction. The logging decision is primarily made by two orthogonal filters, one based exclusively on DCH data and the other based only on EMC data.

The drift chamber filters select events with one tight (high $p_{\mathrm{t}}$ ) track or two loose tracks originating from the IP, respectively. To account for the fact that the IP is not exactly at the origin, track selection is based on its $x-y$ closest approach distance to the IP, $d_{0}^{I P}$, and $z_{0}^{I P}$, the corresponding $z$ coordinate for that point. The IP position is a fixed location close to the average beam position over many months. The high $p_{\mathrm{t}}$ track is required to have a transverse momentum of $p_{\mathrm{t}}>$ $600 \mathrm{MeV} / c$ and to satisfy a vertex condition defined as $\left|d_{0}^{I P}\right|<1.0 \mathrm{~cm}$, and $\left|z_{0}^{I P}-z_{I P}\right|<7.0 \mathrm{~cm}$. Two tracks are accepted with $p_{\mathrm{t}}>250 \mathrm{MeV} / c$ and a somewhat looser vertex condition defined as $\left|d_{0}^{I P}\right|<1.5 \mathrm{~cm},\left|z_{0}^{I P}-z_{I P}\right|<10.0 \mathrm{~cm}$.

Two calorimeter cluster filters select events with either high energy deposits or high cluster multiplicity. Each filter also requires a high effective mass calculated from the cluster energy sums and the energy weighted centroid positions of all clusters in the event assuming massless particles. The first filter requires at least two clusters of $E_{C M}>350 \mathrm{MeV}$ (c.m. system energy) and event mass greater than $1.5 \mathrm{GeV}$; the second filter requires at least four clusters, and an event mass greater than $1.5 \mathrm{GeV}$.

At current luminosities, the output of both the DCH and EMC filters is dominated by Bhabha events, which need to be rejected. This is accomplished by a Bhabha veto filter that selects one-prong (with only a positron in the backward part of the detector) and two-prong events (with both $e^{+}$and $e^{-}$detected). Stringent criteria on EMC energy deposits are imposed, relying on the track momenta and on $E / p$. The two-prong veto requires either colinearity between the tracks in the c.m. system or an acolinearity that is consistent with initial state radiation (ISR). 
For purposes of calibration and offline luminosity measurements, Bhabha, radiative Bhabha, $\gamma \gamma$ final state, and cosmic ray events are flagged. The output rate of flagged Bhabha events is adjusted to generate an approximately flat distribution of events in polar angle. Radiative Bhabha events are identified by selecting two-prong events with missing energy and requiring an EMC cluster in the direction of the missing momentum. Events with two high energy clusters, back-to-back in the c.m. system select the $e^{+} e^{-} \rightarrow \gamma \gamma$ process. The cosmic ray selection is DCH-based and requires two back-to-back tracks in the laboratory frame with nearly equal impact parameters and curvature. A significant background from ISR Bhabha events faking this topology is removed using the same kinematic constraints used in the two-prong veto.

The online luminosity monitoring and energy scale monitoring are performed in L3. A trackbased lepton-pair selection with a well known efficiency monitors the luminosity. Hadronic filters for selection of continuum and $B \bar{B}$-enriched samples monitor the energy scale. The latter two categories are distinguished by an event shape selection using a ratio of Fox-Wolfram moments [114]. The ratio of the $B \bar{B}$-enriched sample to the luminosity is a sensitive measure of relative position on the $\Upsilon(4 S)$ peak and thereby monitors the beam energies.

\subsection{Level 3 Performance and Operational Experience}

The L3 trigger efficiency for Monte Carlo simulated events are tabulated in Table 18 for events passing Level 1. High efficiencies are independently achieved for the DCH and EMC based filters applied to simulated hadronic events. The comparison between data and Monte Carlo L3 trigger pass fractions for the various filters also show good agreement when requiring tracking, and EMC based hadronic event selections in turn.

An example of the event display used for online trigger monitoring is shown in Figure 88. L3 reconstructed tracks and EMC clusters are shown together with the L1 and L3 trigger line states for the event. The left column lists the L1 trigger lines and their states: on (1); off $(0)$; or on
Table 19

Composition of the L3 output at a luminosity of $2.6 \times 10^{33} \mathrm{~cm}^{-2} \mathrm{~s}^{-1}$.

\begin{tabular}{lr}
\hline \hline Event type & Rate $(\mathrm{Hz})$ \\
\hline Hadrons, $\tau \tau$, and $\mu \mu$ & 16 \\
Other QED, 2-photon events & 13 \\
Unidentified Bhabha backgrounds & 18 \\
Beam-induced backgrounds & 26 \\
\hline Total physics accept & $\mathbf{7 3}$ \\
\hline \hline Calibration Bhabhas $\left(e^{+} e^{-}\right)$ & 30 \\
$\gamma \gamma$, Radiative Bhabhas $\left(e^{+} e^{-} \gamma\right)$ & 10 \\
Random triggers and cosmic rays & 2 \\
L1,L3 pass through diagnostics & 7 \\
\hline Total calibration/diagnostics & $\mathbf{4 9}$ \\
\hline \hline
\end{tabular}

but ignored due to prescale factor $(-1)$. The right column shows the same information for the L3 trigger lines.

For a typical run on the $\Upsilon(4 S)$ peak with an average luminosity of $2.6 \times 10^{33} \mathrm{~cm}^{-2} \mathrm{~s}^{-1}$, the L3 event composition is tabulated in Table 19. The desired physics events contribute $13 \%$ of the total output while the calibration and diagnostic samples comprise $40 \%$.

The L3 executable currently takes an average processing time of $8.5 \mathrm{~ms}$ per event per farm computer. A Level 1 input rate of $2700 \mathrm{~Hz}$ saturates the Level 3 processors, well above the $2 \mathrm{kHz}$ design requirement. At this input rate the L3 process consumes $\sim 72 \%$ of the CPU time, the rest is spent in OEP, including the network event builder and in the operating system kernel.

\subsection{Summary and Outlook}

Both the L1 and L3 trigger systems have met their original design goals at a luminosity of $3 \times 10^{33} \mathrm{~cm}^{-2} \mathrm{~s}^{-1}$. The triggering efficiencies for $B \bar{B}$ events generally meet the $99 \%$ design goal for both L1 and L3. The orthogonal triggers based on DCH-only and EMC-only information have successfully delivered stable and measurable overall trigger efficiency. The current system also provides a solid foundation for an upgrade path to luminosities of $10^{34} \mathrm{~cm}^{-2} \mathrm{~s}^{-1}$ or more.

Short-term L1 trigger improvements will pri- 


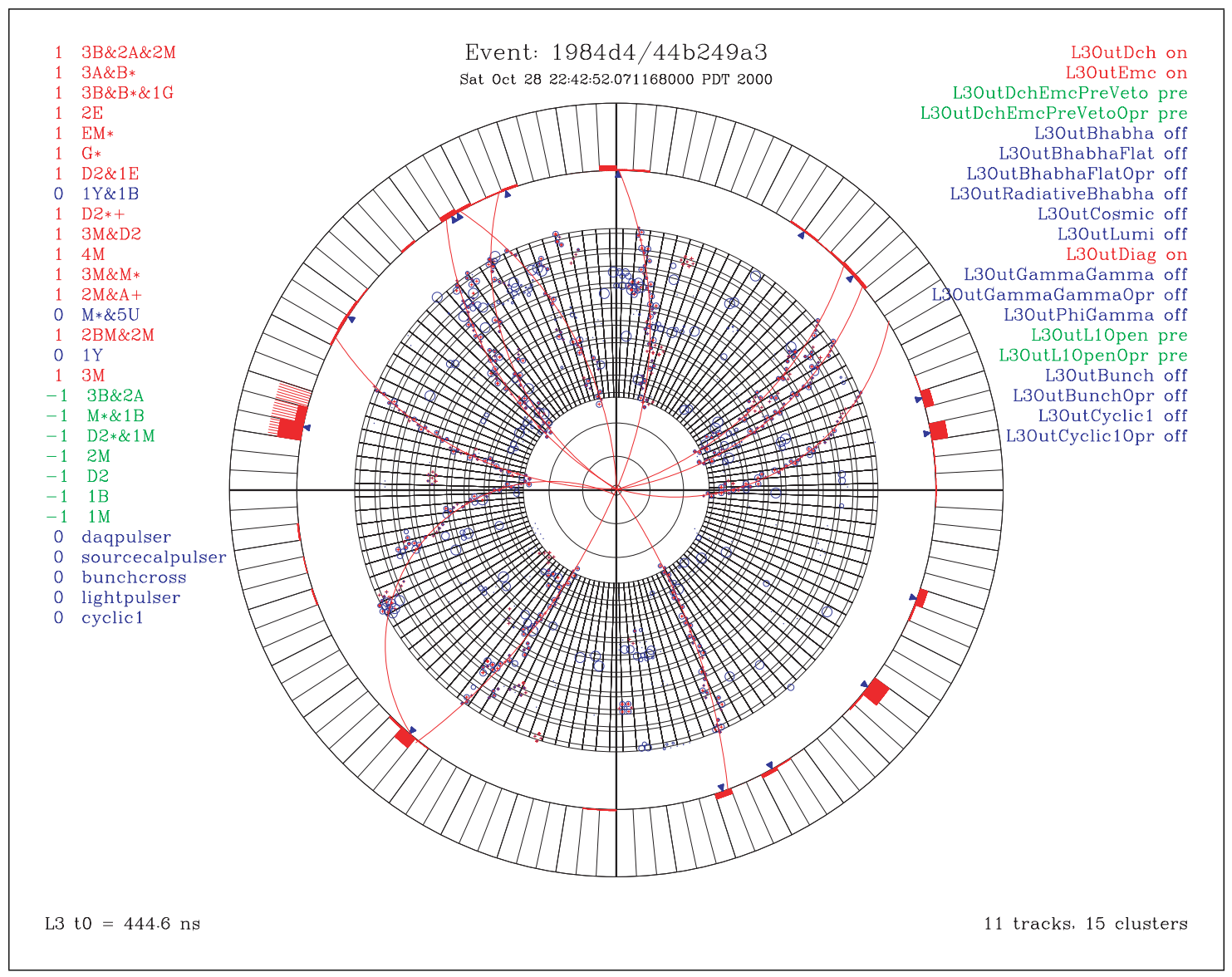

Figure 88. A Level 3 event display. The small circles and small crosses in the DCH volume are DCH hits and TSF segment hit wires respectively. The filled EMC crystals represent energy deposit (full crystal depth $=2 \mathrm{GeV}$ ) from Level $3 \mathrm{EMC}$ clusters while the small triangles just inside the EMC indicate the location of the cluster centroid.

marily come from further background rejection, afforded by algorithm refinements and upgrades of the DCT. This is essential for reducing the load on the DAQ and L3. The new PTD algorithm will effectively narrow the track $d_{0}$ acceptance window, while a new BLT algorithm will narrow the track $z_{0}$ acceptance.

For the longer term future, a major DCT upgrade is planned. By adding the stereo layer information, a $z_{0}$ resolution of $4 \mathrm{~cm}$ is expected, allowing for an efficient rejection of beam-induced background beyond $z= \pm 20 \mathrm{~cm}$.
Future improvements for L3 will also emphasize background rejection. Improvements in the L3 IP track filter are expected to further reduce beam-induced background to about one third of current levels. The physics filter algorithms will be tuned and improved, primarily for rejecting Bhabha, QED, and two-photon events. Improvements in the L3 tracking algorithms are expected to lower the $p_{\mathrm{t}}$ thresholds below $250 \mathrm{MeV} / c$. A moderate CPU upgrade for the L3 online farm will be sufficient to keep up with luminosities of $\sim 10^{34} \mathrm{~cm}^{-2} \mathrm{~s}^{-1}$. 
Table 18

L3 trigger efficiency (\%) for various physics processes, derived from Monte Carlo simulation.

\begin{tabular}{lrrrrrr}
\hline \hline L3 Trigger & $\varepsilon_{B \bar{B}}$ & $\varepsilon_{B \rightarrow \pi^{0} \pi^{0}}$ & $\varepsilon_{B \rightarrow \tau \nu}$ & $\varepsilon_{c \bar{c}}$ & $\varepsilon_{u d s}$ & $\varepsilon_{\tau \tau}$ \\
\hline 1 track filter & 89.9 & 69.9 & 86.5 & 89.2 & 88.2 & 94.1 \\
2 track filter & 98.9 & 84.1 & 94.5 & 96.1 & 93.2 & 87.6 \\
Combined DCH filters & 99.4 & 89.1 & 96.6 & 97.1 & 95.4 & 95.5 \\
\hline 2 cluster filter & 25.8 & 91.2 & 14.5 & 39.2 & 48.7 & 34.3 \\
4 cluster filter & 93.5 & 95.2 & 62.3 & 87.4 & 85.5 & 37.8 \\
Combined EMC filters & 93.5 & 95.7 & 62.3 & 87.4 & 85.6 & 46.3 \\
\hline Combined DCH+EMC filters & $>99.9$ & 99.3 & 98.1 & 99.0 & 97.6 & 97.3 \\
\hline Combined L1+L3 & $>99.9$ & 99.1 & 97.8 & 98.9 & 95.8 & 92.0 \\
\hline \hline
\end{tabular}

\section{The Online Computing System}

\subsection{Overview}

The BABAR online computing system comprises the data acquisition chain from the common FEE, through the embedded processors in the data acquisition system and the L3 trigger, to the logging of event data. It also includes those components required for detector and data acquisition control and monitoring, immediate data quality monitoring, and online calibration.

\subsubsection{Design Requirements}

The data acquisition chain was designed to meet the following basic performance requirements. It must support a L1 trigger accept rate of up to $2 \mathrm{kHz}$, with an average event size of $\sim 32$ kbytes and a maximum output (L3 trigger accept) rate of $120 \mathrm{~Hz}$. While performing these functions it should not contribute more than a time-averaged $3 \%$ to deadtime during normal data acquisition.

The online system is also required to be capable of performing data acquisition simultaneously on independent partitions - sets of detector system components - to support calibrations and diagnostics.

Normal detector operation, data acquisition and routine calibrations are performed efficiently and under the control of a simple user interface with facilities for detecting, diagnosing, and recovering from common error conditions.

Following standard practice, the event data ac- quired by the system are subjected to monitoring. Such monitoring is configurable by experts and designed to detect anomalies in the detector systems which, if present, are reported to operators for rapid assessment and, if necessary, corrective action.

Environmental conditions of the detector, such as the state of low and high voltage power, the purity of gas supplies, and the operating conditions of the accelerator, such as beam luminosity and currents, are measured and recorded in a fashion that permits the association with the event data logged. Conditions relevant to data quality are monitored for consistency with specified standards. Operators are alerted if these are not met. Data-taking is inhibited or otherwise flagged if conditions are incompatible with maintaining the quality of the data.

Operational configurations, calibration results, active software version numbers, and routine messages and error messages are also recorded. During data analysis or problem diagnosis, these data help in reconstructing the detailed operating conditions.

\subsubsection{System Components}

The online computing system is designed as a set of subsystems using elements of a common software infrastructure running on a dedicated collection of hardware.

The major subsystems are:

- Online Dataflow (ODF) — responsible for 
communication with and control of the detector systems' front-end electronics, and the acquisition and building of event data from them;

- Online Event Processing (OEP)responsible for processing of complete events, including L3 (software) triggering, data quality monitoring, and the final stages of calibrations;

- Logging Manager (LM) - responsible for receiving selected events sent from OEP and writing them to disk files for use as input to the Online Prompt Reconstruction processing;

- Online Detector Control (ODC) responsible for the control and monitoring of environmental conditions of the detector systems;

- Online Run Control (ORC) - ties together all the other components, and is responsible for sequencing their operations, interlocking them as appropriate, and providing a graphical user interface (GUI) for operator control.

Each of these components, as well as a selection of the common tools which tie them together are described below.

The entire system is coded primarily in the $\mathrm{C}++$ language, with some use of Java for graphical user interfaces. Object-oriented analysis and design techniques have been used throughout. This has been an important feature, enhancing development speed, maintainability, and extensibility.

\subsubsection{Hardware Infrastructure}

The hardware infrastructure for the online system is shown schematically in Figure 89.

The data from the FEEs of the various detector systems are routed via optical fiber links to a set of 157 custom VME Readout Modules (ROMs). These ROMs are grouped by detector system and housed in 23 data acquisition VME crates that are controlled by the ODF software. One ROM in each crate aggregates the data and forwards them for event building to 32 commercial Unix workstations [115] which are part of the online farm. Other farm machines perform data monitoring and calibrations. The crates and farm machines communicate via full-duplex $100 \mathrm{Mbits} / \mathrm{s}$ Ethernet, linked by a network switch - the event builder switch [116]. The ROMs are supported by a boot server providing core and system-specific code and configuration information [117].

The thirty-two online farm machines host the OEP and L3 trigger software. The events accepted by the trigger are logged via TCP/IP to a logging server [117] and written to a disk buffer for later reconstruction and archival storage. Various data quality monitoring processes run on farm machines not used for data acquisition.

Several additional file servers hold the online databases and production software releases. A further set of application servers host the central functions of the various online subsystems. Operator displays are supported by a group of ten console servers [118].

An additional set of $15 \mathrm{VME}$ crates, each with an embedded processor, contain the data acquisition hardware for the detector control subsystem.

All VME crates, the online farm, and all the application and console servers are connected via a switched $100 \mathrm{Mbits} / \mathrm{s}$ Ethernet network distinct from that used for event building, with 1 Gbits/s fiber Ethernet used for the file servers and interswitch links.

\subsubsection{User interaction}

Operator control of the online system is achieved primarily through a custom Motif GUI for run control and an extensive hierarchy of displays for detector control, including control panels, strip charts and an alarm handler. An electronic logbook is made available through a Web browser interface. These and other GUIs are organized across seventeen displays for the use of the experiment's operators. This operator environment provides for basic control of data acquisition, the overall state of the detector, and certain calibration tasks.

Each detector system has developed a set of specialized calibration and diagnostic applications using the tools provided in the online sys- 


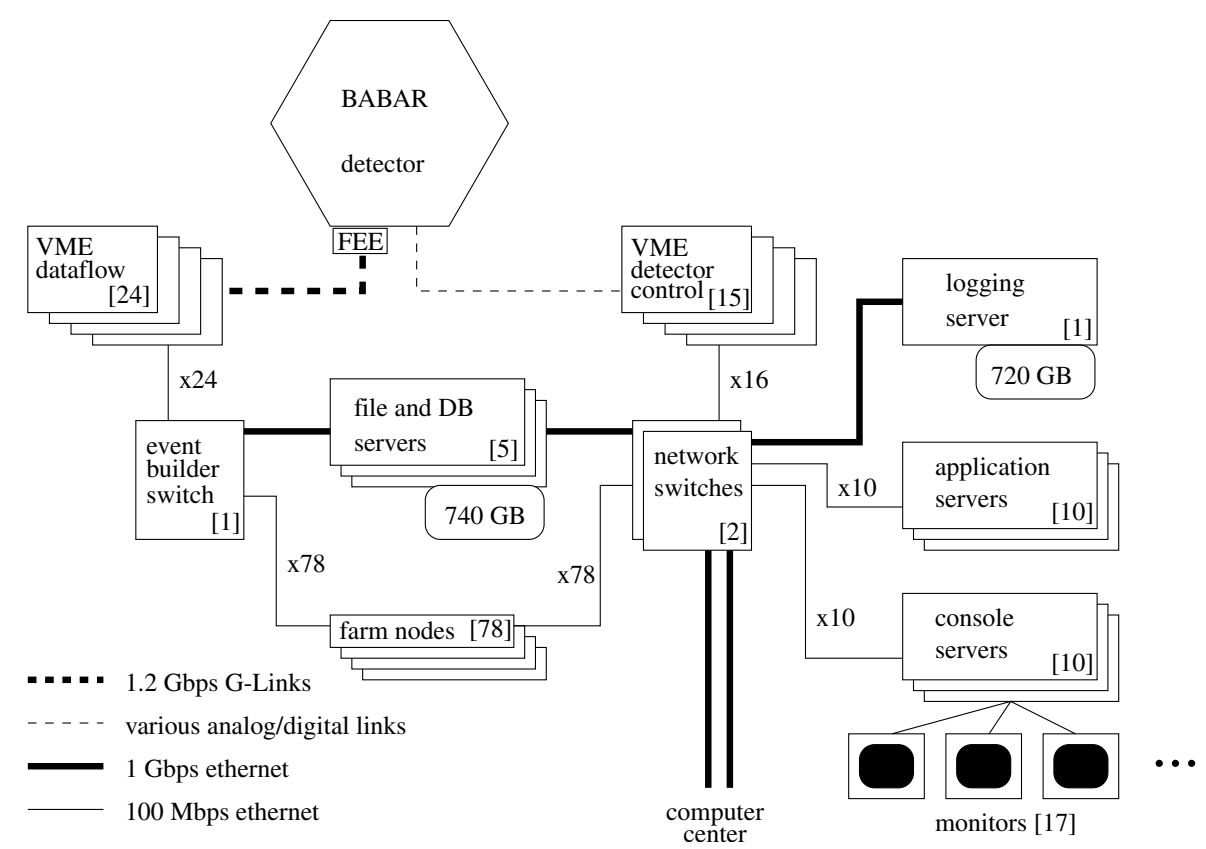

Figure 89. Physical infrastructure of the BABAR online system, including VME crates, computers, and networking equipment.

tem. A subset of these calibrations has been specified to be run once per day, during a ten-minute scheduled beam-off period. The run control logic, combined with the capability for creating partitions, allows calibrations for all detector systems to be run in parallel and provides the operator with basic feedback on the success or failure of each.

\subsection{Online Dataflow}

ODF handles data acquisition and processing from the detector systems' FEE through the delivery of complete events to the online farm [119]. The ODF subsystem receives the L1 trigger outputs, filters and distributes them to the FEE, reads back the resulting data and assembles them into events. It provides interfaces for control of data acquisition, processing and calibration of detector system data, and FEE configuration. Multiple independent partitions of the detector may be operated simultaneously.

Event data acquisition proceeds from a trigger decision formed in the Fast Control and Timing System (FCTS) [120] based on inputs from the L1 trigger. L1 Accepts are distributed, in the full detector configuration, to the $133 \mathrm{ROMs}$ connected via optical fibers to the detector system FEE. These ROMs read and process the data from the FEE. One to ten such ROMs from a single detector system are located in each of the data acquisition VME crates. ODF builds complete events from these ROMs, first collecting the data in each crate into an additional dedicated ROM, and then collecting the data from the 23 of these, across the event builder network switch, into the online farm.

The operation of the system is controlled by ODF software running on one of the application servers, under the direction of run control. A single ROM in the VME crate containing the central FCTS hardware supports the software interface to ODF. The distribution of ROMs by detector system is shown in Table 20. The numbers of ROMs is shown as a sum of those connected directly to the detector FEE, and those used for event building.

All of the ROM CPUs boot via NFS over the 
Table 20

VME crates and ROMs used by ODF

\begin{tabular}{lcc}
\hline $\begin{array}{l}\text { Detector } \\
\text { System }\end{array}$ & $\begin{array}{c}\text { VME } \\
\text { Crates }\end{array}$ & $\begin{array}{c}\text { Readout } \\
\text { Modules }\end{array}$ \\
\hline SVT & 5 & $14+5$ \\
DCH & 2 & $4+2$ \\
DIRC & 2 & $6+2$ \\
EMC & 10 & $100+10$ \\
IFR & 1 & $4+1$ \\
EMT & 1 & $1+1$ \\
DCT & 1 & $3+1$ \\
GLT & 1 & $1+1$ \\
FCTS & 1 & 1 \\
\hline TotalTotal & 24 & 157 \\
\hline
\end{tabular}

event building network from the boot server described above. About 1.5 Mbytes of core ODF code plus another $\sim 4$ Mbytes of detector-specific code are loaded into each ROM. This, along with the booting process, takes about 40 seconds.

The ODF software allows all the components of this heterogeneous system to be represented in a uniform object-oriented application framework. These components are organized into five levels which map closely onto the physical structure.

For each component at each level, its operation is abstracted as a finite state machine. The complete set of these machines is kept coherent by passing messages and data regarding state transitions along the chain of levels. The basic flow of control and data is shown in Figure 90. The mapping of levels to components is as follows:

Control The Unix-based process controlling the operation of each partition and the source of all state transitions except for L1 Accept. It transmits state transition messages over the network to the source level, waiting for acknowledgement of their successful processing by all levels.

Source The FCTS hardware and the software running in the ROM located in the FCTS VME crate. For each partition, its source level receives control level transitions and L1 trigger outputs and distributes them via the FCTS hardware to all ROMs in the VME crates included in the partition. L1 triggers are modeled in the subsystem as an additional, idempotent state transition, $L 1$ Accept, and are treated uniformly with the others wherever possible.

Segment The ROMs connected to the detector FEE, with their ODF and detector systemspecific software. Each segment level ROM receives state transition messages from the source level and runs appropriate core and detector system-specific tasks in response. These tasks include the acquisition of raw data from the FEE in response to L1 Accepts, and feature extraction. Output data resulting from this processing is attached to the transition messages, which are then forwarded over the VME backplane to the fragment level ROM in each crate.

Fragment The per-crate event builder ROMs and software. The single fragment level ROM in each crate aggregates the messages from the crate's segment level ROMs - the first stage of event building - and forwards the combined message to one of the event level Unix nodes.

Event The processes on the online farm nodes receiving complete events and handing them over to OEP for filtering and logging. The ODF event level code aggregates messages, with their attached data, from all the crates in a partitionthe second and final stage of event building. The resulting data may be further processed by user code in the event level, but are normally just passed on to OEP. The control level is notified of the completion of processing of all transitions other than L1 Accept. Both the fragment and event level event builders use a data-driven "push" model, with a back pressure mechanism to signal when they are unable to accept more data.

Test stands of varying complexity are supported. The simplest possible consists of a single Unix machine which runs both control and event level code, with two FCTS modules and a single ROM, running source, segment, and fragment level code, in one VME crate. Configuration is detected at run-time, so the same code that runs in the full system can also run in test stand systems. 


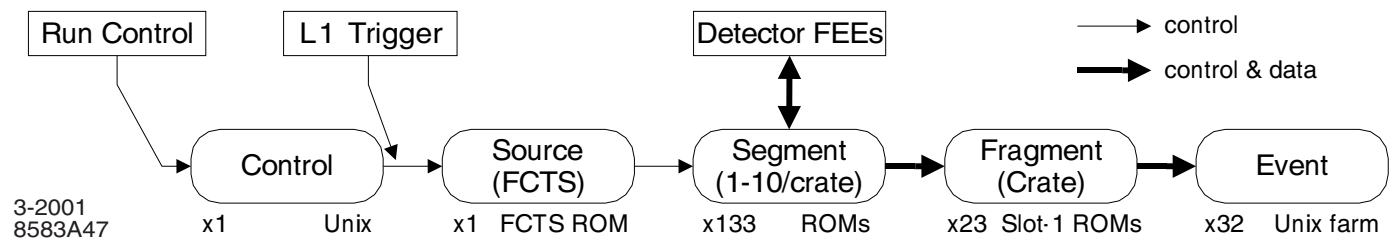

Figure 90. Schematic of the ODF levels, their mapping onto physical components, and the flow of control signals and data between them.

\subsubsection{Control and Source Levels}

The control level sends state transition messages for a partition over the network, using the User Datagram Protocol, UDP [121], to the source level in the single ROM inside the FCTS crate. In the source level, the transition message is sent over VME to an FCTS module which forwards it as a 104-bit $59.5 \mathrm{MHz}$ serial word to all VME crates in the relevant partition. This serial word contains a 56-bit event time stamp (counting at $59.5 \mathrm{MHz}$ ), a 32-bit transition-specific word and additional control bits. L1 Accept transitions and calibration sequences, however, originate in the source level and the same mechanism is used to transmit them through the system.

The FCTS hardware receives the $24 \mathrm{~L} 1$ trigger output lines and eight additional external trigger lines. The FCTS crate is a $9 \mathrm{U}$ VME crate, with a custom P3 backplane on which all the trigger lines are bussed. For each partition, an FCTS module receives these lines. It is configurable with a bit mask specifying the trigger lines enabled for its partition, and an optional prescale factor for each line. A trigger decision is formed for the partition by taking the logical OR of the enabled prescaled lines. Twelve of these modules are installed in the full system, thus setting its maximum number of partitions. A detector system can belong to only one partition at a time.

The FCTS crate receives two timing signals from the accelerator: a $476 \mathrm{MHz}$ clock tied to the RF structure of PEP-II and a $136 \mathrm{kHz}$ fiducial that counts at the beam revolution frequency. The former is divided by eight to create a $59.5 \mathrm{MHz}$ system clock. The fiducial is used to start timing counters and to check the synchronization of the clocks.

There are two types of deadtime in the ODF subsystem. The first arises from the minimum $2.7 \mu$ s spacing between L1 Accept transitions. This restriction simplifies the logic design of the FEE readout, because each signal in the silicon tracker and drift chamber is thus associated with only one L1 Accept. The FCTS hardware enforces this minimum separation between transitions, introducing an irreducible, yet minimal dead time of $0.54 \%$ at $2 \mathrm{kHz}$.

The second type of deadtime arises when all FEE buffers are full and thus unable to accept another event. In a time required to be less than the inter-command spacing, each VME crate in a partition may send back a FULL signal indicating that it is no longer able to process further L1 Accept transitions. The FCTS hardware detects these signals and disables triggering until the FEE are once again prepared to accept data.

An actual L1 Accept signal is only generated from a partition's trigger decision when neither form of dead time is asserted.

\subsubsection{Segment and Fragment Levels}

The segment and fragment levels reside in the 23 detector system VME crates. These are standard 9U crates with a custom P3 backplane.

The 104-bit serial transition messages that leave the source level are received by a FCTS module in each VME crate in a partition. This module in turn forwards these messages to the ROMs in the crate over the custom backplane, along with the $59.5 \mathrm{MHz}$ system clock.

A ROM consists of four components (see Figure 91), a commercial single-board computer (SBC) [122] and three custom boards. The custom boards include: a controller card for receiving FCTS commands and supporting FEE reads and writes; a personality card that transmits com- 


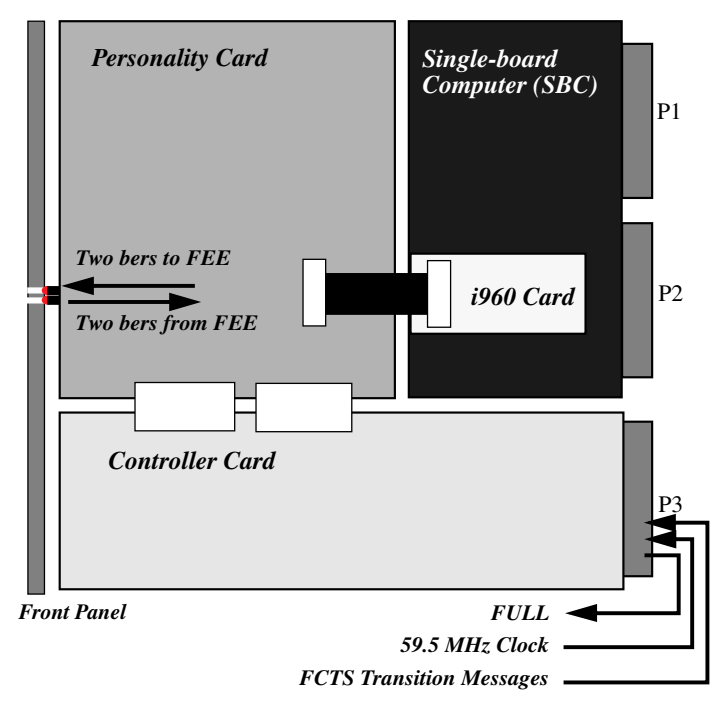

Figure 91. A ROM with a triggered personality card (TPC)

mands to and receives data from the FEE; and a PCI mezzanine card with a $33 \mathrm{MHz}$ Intel i960 I/O processor. The SBCs run the VxWorks [8] operating system with custom code written in $\mathrm{C}++$ and assembly language.

There are two styles of personality cards in the system: triggered (TPC) and untriggered (UPC). UPCs are used only in the EMC system. UPCs accept data continuously from the FEE into a buffer pipeline, at a rate of $3.7 \mathrm{MHz}$. From these samples EMC trigger information is derived and sent over a dedicated serial link to the trigger hardware, providing it with a continuous data stream. An L1 Accept causes up to 256 samples of the raw data stream to be saved to an intermediate memory on the UPC.

A TPC (used in all other systems) reads out FEE data only when an L1 Accept signal is received, again saving it into an intermediate memory. Each detector reads out data in a time window around the trigger signal, large enough to allow for trigger jitter and detector time resolution. For instance, this window is about $500 \mathrm{~ns}$ wide for the SVT. The actual event time within this window is estimated in the L3 trigger soft-
Table 21

Typical event sizes from detector systems

\begin{tabular}{lccc}
\hline Detector & $\begin{array}{c}\text { Hit Size } \\
(\text { bytes })\end{array}$ & $\begin{array}{c}\text { Total Size } \\
(\mathrm{kB})\end{array}$ & $\begin{array}{c}\text { Overhead } \\
(\mathrm{kB})\end{array}$ \\
\hline SVT & 2 & 4.9 & 0.4 \\
DCH & 10 & 4.8 & 0.2 \\
DIRC & 4 & 3.1 & 0.3 \\
EMC & 4 & 9.1 & 3.0 \\
IFR & 8 & 1.2 & 0.2 \\
EMT & - & 1.2 & $<0.1$ \\
DCT & - & 2.7 & 0.1 \\
GLT & - & 0.9 & $<0.1$ \\
\hline Total & & 27.9 & 4.2 \\
\hline
\end{tabular}

ware and then refined offline in the course of full event reconstruction.

FEE commands are sent and data received by the personality cards over uni-directional 1.2 Gbits/s serial optical fiber links [123]. All FEEs provide zero suppression in hardware except in the EMC and IFR. Data are transferred from the personality card to the SBC memory using the i960 as a direct memory access (DMA) engine. This DMA runs at nearly the ideal $133 \mathrm{Mbytes} / \mathrm{s}$ rate of the PCI bus.

The FEEs for various systems are able to buffer data for three to five L1 Accept transitions. The ROM keeps track of the buffer occupancy and sends, when necessary, a FULL signal as previously described. The FULL condition is removed when event reading by the ROM frees sufficient buffer space. This mechanism handles back pressure from any stage of the data acquisition through to data logging by OEP.

The ODF application framework provides uniform software entry points for the insertion of user code at each level of the system. This capability is used primarily at the segment level, for FEE configuration and feature extraction. Table 21 presents typical data contributions from each detector system and the trigger.

Data from the segment level ROMs in a crate are gathered by the fragment level ROM using a chained sequence of DMA operations. The maximum throughput of the fragment level event builder is about $31 \mathrm{Mbits} / \mathrm{s}$. 
In calibrations, ODF may be operated in a mode in which $L 1$ Accept data are not transferred out of the segment level ROMs. This allows for calibration data accumulation at high rates inside the ROMs, not limited by the throughput of the event builders or any downstream software. Completed calibration results are computed, read out, and written to a database.

\subsubsection{Event Level}

For each L1 Accept transition passing through the ODF subsystem, all fragment ROM data are sent to one of the farm machines. The destination is chosen by a deterministic calculation based on the L1 Accept's 56-bit time stamp, available from the FCTS in each ROM. This technique produces a uniform quasi-random distribution and introduces no detectable inefficiency. Events sent to a farm machine still busy with a previous event are held in a buffer to await processing.

All fragment data for an event are sent over the switched $100 \mathrm{Mbits} / \mathrm{s}$ Ethernet event building network to the selected farm machine. The connectionless UDP was chosen as the data transport protocol [124], allowing a flow control mechanism to be tailored specifically to this application. Dropped packets are minimized by the network's purely point-to-point, full duplex switched architecture, and by careful tuning of the buffering in the network switch and other parameters. The rare instance of packet loss is detected by the event builder and the resulting incomplete event is flagged.

The event level provides the standard software entry points for user code. During normal operation, these are used only to transfer events via shared memory to the OEP subsystem for L3 triggering, monitoring, and logging.

\subsubsection{System Monitoring}

It is critical that the clocks of the FEEs stay synchronized with the rest of the system. Each FEE module maintains a time counter which is compared to the time stamp of each L1 Accept in order to ensure that the system remains synchronized. If it becomes unsynchronized, a special synch command can be sent through the FCTS, causing all systems to reset their clocks.
To ensure that the data from the correct event is retrieved from the FEEs, a five-bit counter is incremented and sent from the FCTS to the FEEs with each L1 Accept. These bits are stored in the FEEs along with the data and are compared on read-back. If they disagree, a special clearreadout command is sent which resynchronizes ROM buffer pointers with FEE buffer pointers.

All transitions, including $L 1$ Accept, are logged in a 4 kbytes-deep by 20 byte-wide FIFO as they pass through the FCTS crate. The transition type, the event time stamp, a bit list of the trigger lines contributing to the decision, and the current FULL bit list from all VME crates are recorded in this FIFO. There are also scalers which record delivered and accepted luminosity, deadtime due to the $2.7 \mu \mathrm{s}$ minimum inter-command spacing, deadtime caused by VME crates being FULL and triggers on each line. These FIFOs and scalers are read out by the FCTS ROM, which then transmits the data to monitoring programs that calculate quantities such as luminosity, deadtime and trigger rates. The UDP multicast protocol [125] is used to allow efficient simultaneous transmission of data to multiple clients.

To provide diagnostics, a system which multicasts additional performance information on demand from each CPU, typically at $1 \mathrm{~Hz}$, is used. This information is currently received by a single client on one of the Unix application servers and archived. It can be retrieved subsequently to investigate any unusual behaviour observed in the system.

\subsection{Online Event Processing}

The online event processing (OEP) subsystem provides a framework for the processing of complete events delivered from the ODF event builder [126]. The L3 software trigger operates in this framework, along with event-based data quality monitoring and the final stages of online calibrations. Figure 92 shows the basic flow of data in the OEP subsystem.

The OEP subsystem serves as an adapter between the ODF event builder interface and the application framework originally developed for the offline computing system. Raw data delivered from the ODF subsystem are put into an object- 
oriented form and made available through the standard event data analysis interface.

The use of this technique permits the L3 trigger and most of the data quality monitoring software to be written and debugged within the offline environment. This software is decomposed into small, reusable units-modules, pluggable software components in the framework - many of which are shared among multiple applications.

The OEP interfaces allow user applications to append new data blocks to the original raw data from ODF. The results of L3 event analysis are stored in this manner so that the trigger decision and the tracks and calorimeter clusters on which it is based may be used in later processing, such as reconstruction and trigger performance studies.

Histograms and other monitoring data are accumulated across the farm. A distributed histograming package (DHP) [127] was developed to provide networked clients with a single view of histograms and time history data. This data is summed across all nodes via CORBA-based communication protocols $[128,129]$.

The fast monitoring system provides automated comparisons of monitoring data against defined references. Statistical comparisons of live histograms, or the results of fits to reference histograms, analytic spectra, or nominal values of fit parameters may be performed at configurable time intervals. Comparison failures, tagged with configurable severity levels based on the confidence levels of the comparisons, are displayed to operators and logged in the common occurrence database, described below.

The Java Analysis Studio (JAS) package [130] previously developed at SLAC was enhanced with the ability to serve as a DHP client. It is used for viewing of monitoring data. This feature was implemented by devising a Java server that adapts the DHP protocol to the native JAS data protocol.

In addition to the primary triggering and monitoring functions carried out on 32 online farm machines, OEP provides a "trickle stream" protocol that allows networked clients to subscribe to a sampling of the event data. This scheme provides support for event displays and additional detailed data quality monitoring ("Fast Monitor- ing"). A dedicated operator console supports the JAS-based data quality monitoring system. This console is used to display histograms from Fast Monitoring and the L3 trigger processes, along with any error conditions detected by the automatic histogram analysis facility.

\subsection{Data logging}

Events selected by the L3 trigger algorithms in OEP are retained for subsequent full reconstruction. The events are sent from the 32 OEP nodes via TCP/IP to a single multithreaded process, the Logging Manager (LM), running on the logging server. The LM writes these data to RAID storage arrays in a format specific to OEP. Data from all 32 nodes are combined into a single file for each data-taking run (typically two to three hours of data acquisition, resulting in files of about $15-20$ Gbytes in size).

Completed data files are copied to the SLAC High Performance Storage System (HPSS) [131] system for archiving to tape. Within eight hours of data acquisition these files are retrieved from HPSS for event reconstruction. The data files are also retrievable for other tasks such as detector system hardware diagnostics and offline tests of the L3 trigger algorithms.

\subsection{Detector Control}

\subsubsection{Design Principles}

The Experimental Physics and Industrial Control System, EPICS [7], was selected to provide the basis for the ODC subsystem. This provides direct connection to the electrical signals of the power supplies and other hardware, with sufficient monitoring and control to allow commissioning, fault diagnosis, and testing. A summary of monitoring and control points is presented in Table 22 .

Beyond the writing of custom drivers, only minor additions or changes were required to EPICS. EPICS and the additional BABAR-specific software are written in the $\mathrm{C}$ language.

Detector-wide standard hardware was adopted to ease development and maintenance. The standard ODC crate is a $6 \mathrm{U}$ VME chassis containing a single board computer [132] serving as an EPICS input/output controller (IOC). Fifteen 


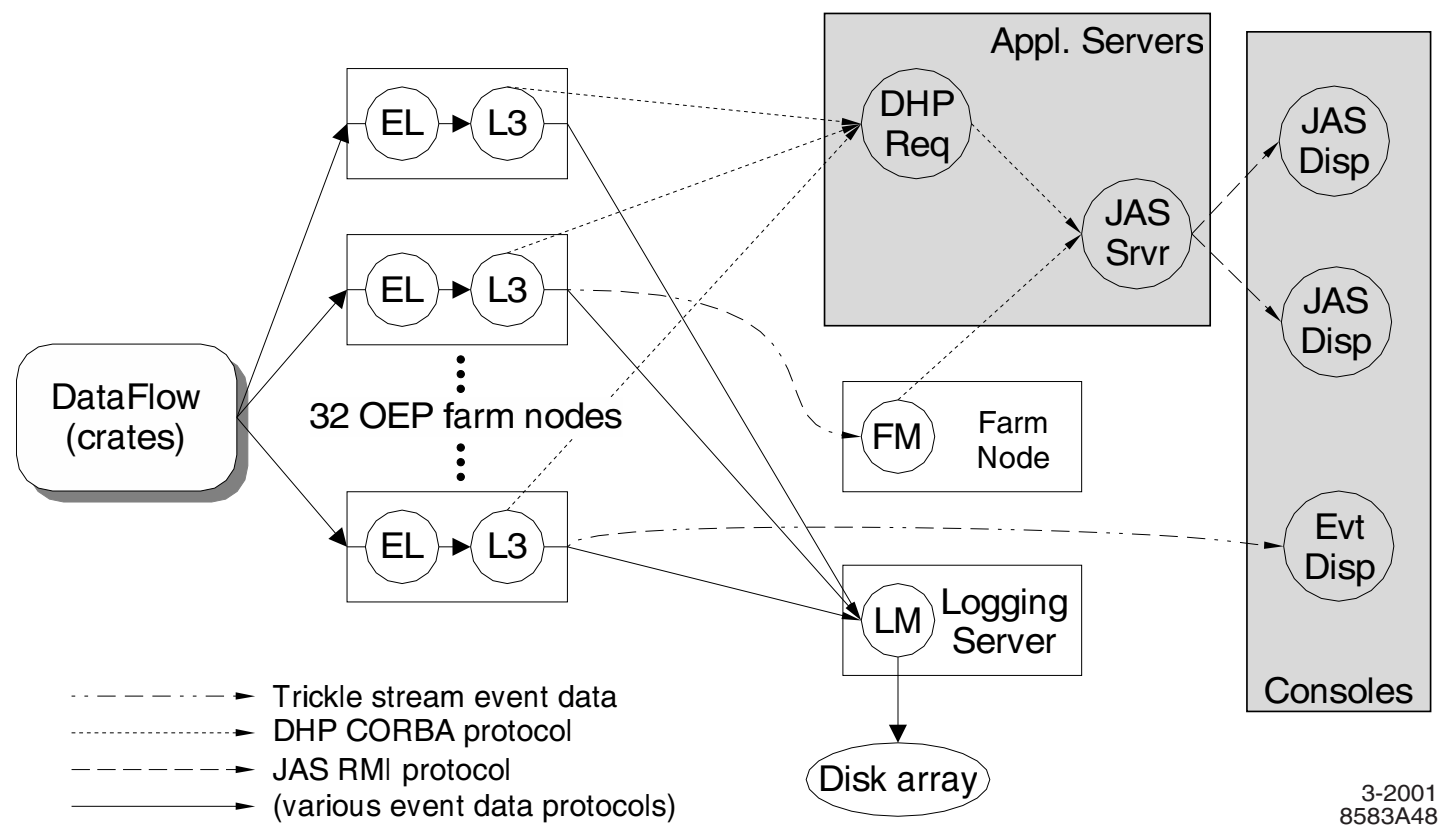

Figure 92. Flow of data in the OEP subsystem: ODF event level (EL) and L3 trigger processes on each OEP node; the Logging Manager (LM) on the logging server; the DHP "requestor" process that combines histograms from all 32 L3 processes; one instance of a Fast Monitoring (FM) process with DHP histograms; the Java server that makes DHP histograms available to JAS clients; two such clients, and one event display for the L3 trigger. OEP-specific data transport protocols are identified.

Table 22

ODC Distribution by system of approximately 12,000 recorded monitor channels

\begin{tabular}{lrrrrrr}
\hline & SVT & DCH & DIRC & EMC & IFR & Central \\
\hline \hline Radiation Dose & 12 & 8 & 22 & 116 & - & - \\
Data Rate & - & - & 12 & - & 1612 & 20 \\
Temperature & 208 & 87 & 36 & 506 & 146 & 100 \\
Humidity 4 & 5 & 12 & 7 & - & - & 2 \\
Magnetic Field - & - & 8 & - & - & - & 50 \\
Position & 30 & 22 & - & - & - & - \\
Gas System & 4 & 115 & 12 & 1 & 32 & - \\
Fluid System & 20 & 2 & 18 & 18 & 20 & 90 \\
Liquid Source & - & - & - & 12 & - & 3 \\
HV System & 2080 & 1299 & 672 & 24 & 1574 & - \\
LV System (non-VME) & - & 62 & 1080 & 442 & 94 & - \\
VME Crates & 5 & 2 & 12 & 12 & 8 & 1500 \\
CAN Micro Controller & 48 & 40 & 3 & 47 & 45 & - \\
Finisar Monitor & 28 & 12 & 12 & - & - & - \\
\hline System Totals & 2439 & 1654 & 1899 & 1185 & 3531 & 1765 \\
\hline \hline
\end{tabular}


such crates are used in the experiment. EPICS is fully distributed. For example, each IOC supplies its own naming service, notify-by-exception semantics, and processing. The IOCs boot from a dedicated server.

Analog data are either digitized by modules within the crates or, more commonly, on digitizer boards located directly on the detector. In the latter case, the CANbus standard [133] is used for the transport of signals to and from the detector. A custom "general monitoring board" (GMB) [134] was developed to interface CANbus to the on-detector electronics. The GMB contains a microcontroller, an ADC, multiplexors, and operational amplifiers. It can digitize up to 32 signals.

\subsubsection{User Interface}

The operator view of this part of the control system is via screens controlled by the EPICS display manager (DM). Dedicated control and display panels were developed using DM for each of the detector systems, using common color rules to show the status of devices. A top-level panel for ODC summarizes the status of all systems and provides access to specialized panels.

The EPICS alarm handler with some BABARspecific modifications is used to provide operators with audible and color-coded alarms and warnings in a hierarchical view of all the systems and components. Conditions directly relevant to personnel or detector safety are further enforced by hardware interlocks, the status of which are themselves displayed in a set of uniform EPICS screens, in the alarm handler, and on an alarm annunciator panel.

\subsubsection{Interfaces to Other BABAR Software}

A custom $\mathrm{C}++$ layer above EPICS consisting of Component Proxies and Archivers provides for device-oriented state management and archival data collection. This is ODC's interface to the rest of the online system.

The 27 component proxies (CPs), running on a Unix application server, each define a logical component representing some aspect of a detector system or the experiment's central support systems, aggregated from the $\lesssim 10^{5}$ individual EPICS records.

The CPs present a simple finite state machine model as their interface to Run Control. The most important actions available are Configure, on which the $\mathrm{CP}$ accesses the configuration database, retrieving set points for its component's channels, and Begin Run, which puts the CP into the Running state, in which setpoint changes are prohibited and readbacks are required to match settings. While in the Running state, the CP maintains a Runnable flag which reflects that requirement and allows Run Control to ensure that data acquisition is performed only under satisfactory conditions.

The CP's other principal function is to provide an interface for the rest of the online system to the ambient data collected by ODC on the state of the detector hardware and its environment. It is the task of the archiver processes, each paired with a $\mathrm{CP}$, to collect the ambient data, aggregate them and write out histories approximately every hour to the ambient database. These recorded data are associated with times so that they may be correlated with the time stamps of the event data. Data from the archiver processes or from the database may be viewed with a custom graphical browser.

Ambient data typically vary only within a narrow noise range or dead-band. The storage of unnecessary data is avoided by recording only those monitored quantities which move outside of a per-channel dead-band range or across an alarm threshold.

\subsubsection{Integration With the Accelerator}

Close integration between the BABAR detector and the PEP-II accelerator is essential for safe and efficient data collection. Data from the accelerator control system are transferred via EPICS channel access to BABAR for display and storage, managed by a dedicated CP. In turn, background signals from the detector are made available to PEP-II to aid in injection and tuning, minimizing backgrounds, and optimizing integrated luminosity. An important component of this communication is the "injection request" handshake. When the PEP-II operator requests a significant 
change in the beam conditions, such as injection, the request can only procede following confirmation from BABAR. This procedure complements the safety interlocks based on radiation dose monitors.

\subsubsection{Operational Experience}

The ODC subsystem has been operational since the initial cosmic-ray commissioning of the detector and the beginning of data-taking with colliding beams. The core EPICS infrastructure has proven to be very robust. The large size of the subsystem, with its 15 IOCs and $\lesssim 10^{5}$ records, produces heavy but manageable traffic on the experiment's network. The rate of data into the ambient database averages $4.6 \mathrm{Mbytes} / \mathrm{hr}$ or 110 Mbytes/day.

\subsection{Run Control}

The ORC subsystem is implemented as an application of SMI++, a toolkit for designing distributed control systems [135]. Using this software, the BABAR experiment is modeled as a collection of objects behaving as finite state machines. These objects represent both real entities, such as the ODF subsystem or the drift chamber high voltage controller, and abstract subsystems such as the "calibrator," a supervisor for the coordination of online components during detector calibration. The behavior of the objects are described in a specialized language (SML) which is interpreted by a generic logic engine to implement the control system.

The SML descriptions of the objects which make up the experiment simply specify their own states and transitions as well as the connections between the states of different objects. Objects perform actions on state transitions, which may include explicitly commanding transitions in other objects; objects may also be programmed to monitor and automatically respond to changes of state in other objects. Anticipated error conditions in components of the online system are reflected in their state models, allowing many errors to be handled automatically by the system. To reduce complexity, logically related objects are grouped together into a hierarchy of cooperating domains.
The system is highly automated; user input is generally required only to initialize the system, start and stop runs, and handle unusual error conditions. The user communicates with ORC via a configurable Motif-based GUI included in $\mathrm{SMI}++$.

The states and behavior of ORC objects representing external systems are provided by a special class of intermediate software processes called proxies. A proxy monitors its system, provides an abstraction of it to ORC, and receives state transition commands. These commands are interpreted and applied to the underlying hardware or software components, implementing the transitions' actions. The control level of an ODF partition is an example of such a proxy.

Communication between the various proxies and the ORC engines is provided by DIM [136], a fault tolerant "publish and subscribe" communications package based on TCP/IP sockets, allowing ORC to be distributed transparently over a network.

Essential to the operation of the online system is the notion of the Runnable status of its various ODC and data acquisition components, indicating that they are in a state suitable for production-quality data-taking. The ORC logic interlocks data-taking to the logical AND of all components' Runnable status. Whenever this condition is not satisfied, data-taking may not start and any existing run will be paused with an alert sent to the operator.

\subsection{Common Software Infrastructure 12.7.1. Databases}

Five major databases are used by the online system:

1. Configuration Database: This database, implemented using the commercial object-oriented database management system Objectivity [6], allows the creation of hierarchical associations of system-specific configuration data with a single numeric configuration key. This key is distributed to all online components, which can then use it to retrieve from the database all the configuration information they require. Convenient mnemonics are associated with the keys for currently relevant configurations, and may be selected for use 
via the ORC GUI [137].

2. Conditions Database: The Conditions Database is used to record calibration and alignment constants, and the configuration keys in force during data-taking runs. It has the additional feature that the data for a given time interval may be updated as they are refined in the course of improved understanding of the apparatus [138].

The Configuration and Conditions Databases are both made available for reconstruction and physics analysis.

3. Ambient Database: The Ambient Database is used principally by the ODC subsystem to record detector parameters and environmental data at the time they are measured [137].

Both the Ambient and Conditions databases, are implemented using Objectivity, and are based on the notion of time histories of various data associated with the experiment. The history for each item is divided into intervals over which a specific value is consistent.

4. Occurrence (Error) Log: Informational and error messages generated in the online system are routed through the CMLOG system [139] to a central database, from which they are available for operators' realtime viewing or historical browsing, using a graphical tool, as well as for subscription by online components which may require notification of certain occurrences.

5. Electronic Logbook: An Oracle-based [140] logbook is used to maintain the history of the data-taking, organized by runs. It contains information on beam parameters - instantaneous and integrated luminosity, currents, and energiesas well as records of data acquisition parameters such as trigger rates, data volumes, and dead times, and the detector configuration used for a run. The logbook also contains text comments and graphics added by the operations staff.

A number of other databases are used in the online system for various tasks such as indexing logged data files, the repair history of online hardware and spares, and software problem reports.

\subsubsection{Software Release Control and Configuration Management}

All of the online software is maintained in the common BABAR code repository, based on the freely available Concurrent Versions System software, CVS [141].

The online's Unix and VxWorks applications are built and maintained with an extension of the standard BABAR software release tools [142]. At the start of every data-taking run, the identities of the current production software release and any installed patches are recorded; thus it is possible at a later date to reconstruct the versions of online software used to acquire data.

\subsection{Summary and Outlook}

The online system has exceeded its data acquisition performance goals. It is capable of acquiring colliding beam events, with an average size of 28 kbytes, at a $\sim 2500 \mathrm{~Hz}$ L1 trigger rate and reducing this rate in $\mathrm{L} 3$ to the required $\sim 120 \mathrm{~Hz}$ limit. This provides comfortable margins, since under normal beam conditions the L1 trigger rate is $800-1000 \mathrm{~Hz}$.

The system is capable of logging data at much higher rates; the nominal $120 \mathrm{~Hz}$ figure represents a compromise between data volume and its consequential load on downstream processing and archival storage, and trigger efficiency for low multiplicity final states.

During normal data-taking, the online system routinely achieves an efficiency of over $98 \%$, taking both data acquisition livetime and the system's overall reliability into account.

There are several hardware options for enhancing ODF capacity. Currently most ROMs receive more than one fiber from the FEE. These fibers could be distributed over more ROMs to add processing power. There are also commercial upgrade paths for the ROMs' SBC boards available. Crates can be split (up to a maximum total of 32) to create more VME event building bandwidth, as well as more fragment level CPU power and network bandwidth. Gigabit Ethernet connections could also be installed to improve the network event builder's bandwidth.

Various software upgrade options are being investigated, including optimizing the VxWorks 
network drivers and grouping sets of events together in order to reduce the impact of per-event overhead.

Current background projections indicate that fragment level CPU, segment level memory bus bandwidth, and network event building bandwidth are the most likely bottlenecks for future running.

Increases in the L1 trigger rate or in the background occupancy and complexity of events are expected to necessitate additional capacity for OEP, principally for L3 triggering. The online farm machines could be replaced with faster models. More machines could be added, at the expense of increases in coherent loading on various servers and of additional management complexity.

No significant capacity upgrades to the data logging subsystem or to ODC are foreseen at this time.

\section{Conclusions}

During the first year of operation, the BABAR detector has performed close to expectation with a high degree of reliability. In parallel, the PEP-II storage rings gradually increased its performance and towards the end of the first year of datataking routinely delivered close to design luminosity. In fact, the best performance surpassed the design goals, both in terms of instantaneous as well as integrated luminosity per day and month. Of the total luminosity of $23.5 \mathrm{fb}^{-1}$ delivered by PEP-II during the first ten months of the year 2000, BABAR logged more than $92 \%$. The data are fully processed with a delay of only a few hours. They are of very high quality and have been extensively used for physics analysis.

A large variety of improvements to the event reconstruction and detailed simulation are presently being pursued. They include improvements in many aspect of the calibration and reconstruction procedures and software, for example, the calibration and noise suppression in the EMC, and the development of techniques for precision alignment of the SVT and DCH. The latter effort will not only benefit the overall efficiency and precision of the track reconstruction, it will also improve the matching of tracks with signals in the DIRC and EMC. Detailed studies and the full integration of all available information pertinent to the identification of charged and neutral particles are expected to result in better understanding and improved performance of various techniques.

Beyond routine maintenance, minor upgrades and a few replacements of faulty components are currently planned. They include the replacement of SVT modules that are expected to fail in the next few years due to radiation damage, plus a few others that cannot be correctly read out due to broken connections. A large fraction of the RPCs are showing gradually increasing losses in efficiency and plans are being developed for the replacement of the RPC modules over the next few years. Furthermore, 20-25 cm of absorber will be added to the flux return to reduce the hadron misidentification rates.

With the expected increase in luminosity, machine-induced backgrounds will rise. Measures are being prepared to reduce the sources and the impact of such backgrounds on BABAR. Apart from the addition of shielding against shower debris, upgrades to the DCH power supply system and to the DIRC electronics are presently under way. Most important are upgrades to the trigger, both at levels L1 and L3. Specifically, the DCH stereo layer information will be added to allow for a more efficient suppression of background tracks from outside the luminous region of the beam. The L3 processing will be refined so as to reject both backgrounds and high rate QED processes with higher efficiency. In addition, data acquisition and processing capacity will be expanded to meet the demands of higher luminosity.

In summary, the BABAR detector is performing very well under current conditions and is well suited to record data at significantly higher than design luminosity.

\section{Acknowledgements}

The authors are grateful for the tremendous support they have received from their home institutions and supporting staff over the past six years. They also would like to commend their 
PEP-II colleagues for their extraordinary achievement in reaching the design luminosity and high reliability in a remarkably short time. The collaborating institutions wish to thank SLAC for its support and kind hospitality.

This work has been supported by the US Department of Energy and the National Science Foundation, the Natural Sciences and Engineering Research Council (Canada), the Institute of High Energy Physics (P.R. China), le Commisariat à l'Energie Atomique and Institut National de Physique Nucléaire et de Physique des Particules (France), Bundesministerium für Bildung und Forschung (Germany), Istituto Nazionale di Fisica Nucleare (Italy), the Research Council of Norway, the Ministry of Science and Technology of the Russian Federation, and the Particle Physics and Astronomy Research Council (United Kingdom). In addition, individuals have received support from the Swiss National Foundation, the A.P. Sloan Foundation, the Research Corporation, and the Alexander von Humboldt Foundation.

\section{REFERENCES}

1. PEP-II - An Asymmetric B Factory, Conceptual Design Report, SLAC-418, LBL-5379 (1993).

2. The BABAR Collaboration, Letter of Intent for the Study of $C P$ Violation and Heavy Flavor Physics at PEP-II, SLAC-443 (1994).

3. BABAR Technical Design Report, SLAC-R457 (1995).

4. The BABAR Physics Book, Physics at an Asymmetric $B$ Factory, P.F. Harrison, H.R. Quinn, Editors, SLAC-R-504 (1998).

5. The Physics Program of a High-Luminosity Asymmetric B Factory at SLAC, SLAC353 (1989); Proceedings of the Workshop on Physics and Detector Issues for a High Luminosity Asymmetric B Factory at SLAC, SLAC-373 (1991).

6. Objectivity, Inc., Mountain View, CA, USA.

7. L. Dalesio et al., Nucl. Instr. Methods A352 (1994) 179.

8. The VxWorks realtime operating system and Tornado Development interface, Wind River
Systems, Inc., Alameda, CA, USA.

9. T. Glanzman et al., The BABAR Prompt Reconstruction System, Proceedings of the International Conference on Computing in High Energy Physics, Chicago, USA (1998). F. Safai Tehrani, The BABAR Prompt Reconstruction Manager: A Real Life Example of a Constructive Approach to Software Development, submitted to Computer Physics Communications (2000).

10. J. Seeman et al., The PEP-II Storage Rings, SLAC-PUB-8786 (2001), submitted to Nucl. Instr. and Methods .

11. J. Seeman et al., Status Report on PEP-II Performance, Proceeedings of the 7th European Particle Accelerator Conference (EPAC 2000), Vienna, Austria (2000).

12. M. Sullivan, $B$-Factory Interaction Region Designs, Proceedings of the IEEE Particle Accelerator Conference (PAC97), Vancouver, B.C., Canada (1997), SLAC-PUB-7563.

13. S.E. Csorna et al., (CLEO Collaboration), Phys. Rev. 61 (2000) 111101.

14. T. Mattison et al., Background Measurements during PEP-II Commissioning, Proceedings of the IEEE Particle Accelerator Conference (PAC99), New York, NY, USA (1999).

15. W. Kozanecki, Nucl. Instr. Methods A446 (2000) 59.

16. C. Hast et al., Report of the High-Luminosity Background Task Force, BABAR Note 522 (2000).

17. T.I. Meyer et al., Contribution to DPF 2000, Meeting of the Division of Particles and Fields of the American Physical Society, Columbus, OH, USA (2000).

18. B. Camanzi, et al., Nucl. Instr. Methods A 457 (2001) 476.

19. F.Kircher, et al., IEEE Transactions on Applied Superconductivity, 9 \#2 (1999) 847.

20. Opera-2D, Vector Fields, Inc., Aurora, IL, USA; ANSYS by ANSYS Inc., Canonsburg, PA, USA; Mermaid (C)1994) by Sim Limited, Novosibirsk, Russia.

21. A. Onuchin et al., Magnetic Field Calculation in the BABAR Detector, BABAR Note 344 (1996). 
22. L. Keller et al., Magnetic Field Calculation in the BABAR Detector, BABAR Note 370 (1997).

23. Kawasaki Heavy Industries (KHI), Kobe, Japan.

24. Europa Metali SpA, Fornaci di Barga, Italy. Alcatel Swiss Cable (now Nexans), Cortaillod, Switzerland.

25. Solenoid cool-down and cryogenic Helium is supplied by a modified Linde TCF-200 liquefier/refrigerator.

26. Ansaldo Energia, Genova, Italy.

27. The solenoid was shipped from Genova on a C5-B transport plane of the US Air Force.

28. E. Antokhin et al., Nucl. Instr. Methods A432 (1999) 24.

29. C. Newman-Holmes, E.E. Schmidt, R. Yamada, Nucl. Instr. Methods A274 (1989) 443.

30. Sentron model 2MR-4A/3B-14B25-20 (2element) and, AMR-3B-14B25-20 (1-element) Hall Probes, GMW Associates, San Carlos, CA, USA.

31. Metrolab model PT 2025 Telsameter with model 1060 NMR probe, Metrolab Instruments, SA, CH-1228, Geneva, Switzerland.

32. A. Boyarski et al., Field Measurements in the BABAR Solenoid, BABAR Note 514 (2000).

33. UBE Industries, Japan. see also [34]

34. C. Bozzi et al., Nucl. Instr. Methods A447 (2000) 20.

35. D. Barbieri et al., Nuo. Cim. A112 (1999) 113.

36. MICRON Semiconductor Ltd., Lancing, U.K.

37. L. Bosisio, INFN Trieste, Italy, private communication.

38. G. Della Ricca et al., Nucl. Instr. Methods A409 (1998) 258.

39. V. Re et al., Nucl. Instr. Methods A409 (1998) 354.

40. J. Beringer et al., The Data Transmission System for the BABAR Silicon Vertex Tracker, BABAR Note 518 (2000).

41. R. Claus et al., SLAC-PUB-8134 (1999).

42. CAEN, Costruzioni Apparecchiature Elettroniche Nucleari, Viareggio, Italy.

43. Medelec Mechanical, Puidoux, Switzerland.

44. Celenex 3300-2 polyester thermoplastic with $30 \%$ silica glass fiber.

45. E. Borsato et al., Nucl. Instr. Methods A451 (2000) 414.
46. Luma Metall AB, Kalmar, Sweden.

47. California Fine Wire, Grover Beach, CA, USA.

48. A. Berenyi et al., IEEE Trans. Nucl. Sci. 46 (1999) 348; ibid. 46 (1999) 928.

49. J. Albert et al., IEEE Trans. Nucl. Sci. 46 (1999) 2027; A. Bouchan et al., Nucl. Instr. Methods A409 (1998) 46; G. Sciolla et al., Nucl. Instr. Methods A419 (1998) 310.

50. D. Dorfan et al., Nucl. Instr. Methods A409 (1998) 310.

51. S.F. Dow et al., IEEE Trans. Nucl. Sci. 46 (1999) 785.

52. Y. Karyotakis, D. Boutigny, LAPP Annecy, France, private communication.

53. Garfield: Simulation of Gaseous Detectors, CERN Program Library (1992).

54. P. Billoir, Nucl. Instr. Methods A225 (1984) 225.

55. D.N. Brown, E.A. Charles, D.A. Roberts, The BABAR Track Fitting Algorithm, Proceedings of CHEP 2000, Padova, Italy (2000).

56. B.N. Ratcliff, SLAC-PUB-5946 (1992); B. N. Ratcliff, SLAC-PUB-6067 (1993); P. Coyle et al., Nucl. Instr. Methods A343 (1994) 292.

57. Spectrosil is a trademark of TSL Group PCL, Wallsend, Tyne on Wear, NE28 6DG, England; Sold in the USA by Quartz Products Co., Louisville, KY, USA.

58. I. Adam et al., IEEE Trans. Nucl. Sci. 45 No. 3 (June) 657; I. Adam et al., ibid 450; J. Cohen-Tanugi, M. C. Convery, B. N. Ratcliff, X. Sarazin, J. Schwiening, J. Va'vra, SLAC-JOURNAL-ICFA-21, ICFA Instrumentation Bulletin, Fall 2000 Issue.

59. M. Benkebil et al., Nucl. Instr. Methods A442 (2000) 364.

60. Boeing Optical Fabrication, Albuquerque, NM, USA.

61. Electron Tubes Limited (formerly: Thorn EMI Electron Tubes), Ruislip, Middlesex, England.

62. P. Bourgeois, M. Karolak, G. Vasseur, Nucl. Instr. Methods A442 (2000) 105.

63. Epoxy Technology, Inc., Billerica, MA, USA.

64. J. Va'vra, Nucl. Instr. Methods A453 (2000) 262.

65. P. Bailly et al., Nucl. Instr. Methods A432 
(1999) 157.

66. J. Ardelean et al., LAL-RT-97-04 (1997).

67. P. Bailly et al., Nucl. Instr. Methods A433 (1999) 432.

68. The micro-controller is a MC68HC05x32, Motorola Inc., Schaumburg, IL, USA.

69. I. Adam et al., SLAC-PUB-8783 (2001), to be published in IEEE Trans. Nucl. Sci.

70. R. Aleksan et al., Nucl. Instr. Methods A397 (1997) 261.

71. T. Swarnicki, Performance of the CLEO-II CsI(Tl) Calorimeter, Proceedings of Workshop on B Factories, Stanford, CA, USA (1992).

72. R.J. Barlow et al., Nucl. Instr. Methods A420 (1999) 162.

73. Aldrich-APL, Urbana, IL, USA. Chemetall GmbH, Frankfurt, Germany.

74. Shanghai Institute of Ceramics, Shanghai, P.R.China; Beijing Glass Research Institute, Beijing, P.R.China; Hilger Analytical, Margate, Kent, UK; Crismatec, Nemours, France; Amcrys-H, Kharkov, Ukraine.

75. TYVEK, registered trademark of E.I. DuPont de Nemours \& Co., Wilmington, DE, USA.

76. G. Dahlinger, Aufbau und Test eines Kalorimeter-Prototyps aus $\mathrm{CsI}(\mathrm{Tl})$ zur Energie- und Ortsmessung hochenergetischer Photonen, Ph.D Thesis, Technische Universität Dresden, Germany, (1998).

77. EPILOX A17-01 manufactured by Leunaer Harze GmbH, Leuna, Germany.

78. J. Brose, G. Dahlinger, K.R. Schubert, Nucl. Instr. Methods A417 (1998) 311;

C. Jessop et al., Development of Front End Readout for the BABAR $\mathrm{CsI}(\mathrm{Tl})$ Calorimeter, BABAR Note 216 (1995);

C. Jessop et al., Development of Direct Readout for CsI Calorimeter, BABAR Note 270 (1995).

79. NE-561 manufactured by Nuclear Enterprises, Sighthill, Edinburgh, Scotland.

80. The calibration procedure employed in this measurement introduces a dependency of the light yield on the shaping time of the preamplifier. When connected to the actual frontend electronics in the BABAR detector, the signal is reduced by a factor 1.29 .
81. Fluorinert (polychlorotrifluoro-ethylene) is manufactured by 3M Corporation, St. Paul, MN, USA.

82. S-2744-08 PIN diode by Hamamatsu Photonics, K. K., Hamamatsu City, Japan. Dark current $<5 n A$, capacitance $<105 p F$ at the depletion voltage of $70 \mathrm{~V}$.

83. J. Harris, C. Jessop, Performance Tests of Hamamatsu 2774-08 Diodes for the $B A B A R$ Electromagnetic Calorimeter Front End Readout and Proposal for Reliability Issues, BABAR Note 236 (1995).

84. G. Haller, D Freytag, IEEE Trans. Nucl. Sci. 43 (1996) 1610.

85. C. Jessop, Reliability Issues for the $B A B A R$ $\mathrm{CsI}(\mathrm{Tl})$ Calorimeter Front End Readout, BABAR Note 217 (1995).

86. S. Menke, Offline Correction of NonLinearities in the $B A B A R$ Electromagnetic Calorimeter, BABAR Note 527 (2000).

87. F. Gaede, D. Hitlin, M. Weaver, The Radioactive Source Calibration of the BABAR Electromagnetic Calorimeter, BABAR Note 531 (2001);

J. Button-Shafer et al., Use of Radioactive Photon Sources with the BABAR Electromagnetic Calorimeter, BABAR Note 322 (1996).

88. R. Müller-Pfefferkorn, Die Kalibration des elektromagnetischen $\mathrm{CsI}(\mathrm{Tl})$-Kalorimeters des BABAR-Detektors mit Ereignissen der Bhabha-Streuung, Ph.D Thesis, TUDIKTP/01-01, Technische Universität Dresden, Germany (2001).

89. GEANT Detector Description and Simulation tool, CERN Program Library, Long Writeup W5013 (1994).

90. S. Menke et al., Calibration of the BABAR Electromagnetic Calorimeter with $\pi^{0} \mathrm{~s}$, BABAR Note 528 (2000).

91. J. Bauer, Kinematic Fit for the EMC Radiative Bhabha Calibration, BABAR Note 521 (2000).

92. M. Kocian, Das Lichtpulsersystem des elektromagnetischen $\mathrm{CsI}(\mathrm{Tl})$-Kalorimeters des BABAR-Detektors, Ph.D Thesis, TUDIKTP/00-03, Technische Universität Dresden, Germany (2000).

93. P.J. Clark, The BABAR Light Pulser System, 
Ph.D Thesis, University of Edinburgh, UK (2000);

B. Lewandowski, Entwicklung und Aufbau eines Lichtpulsersystems für das Kalorimeter des BABAR-Detektors, Ph.D Thesis, RuhrUniversität Bochum, Germany (2000).

94. B. Brabson et al., Nucl. Instr. Methods A332 (1993) 419.

95. S. Otto, Untersuchungen zur Ortsrekonstruktion electromagnetischer Schauer, Diplomarbeit, Technische Universität Dresden, Germany (2000).

96. R. Santonico, R. Cardarelli, Nucl. Instr. Methods A187 (1981) 377.

97. F. Anulli et al., Nucl. Instr. Methods A409 (1998) 542.

98. A. Calcaterra et al., Performance of the BABAR RPCs in a Cosmic Ray Test, Proceedings of the International Workshop on Resistive Plate Chambers and Related Detectors, Naples, Italy (1997).

99. N.Cavallo et al., Nucl. Phys. B(Proc. Suppl.) 61B (1998) 545; N. Cavallo et al., Nucl. Instr. Methods A409 (1998) 297.

100.HV system SY-127, Pod Models A300-P and A300-N by CAEN, Viareggio, Italy.

101.G. Crosetti et al., Data Acquisition System for the RPC Detector of BABAR Experiment, Proceedings of the International Workshop on Resistive Plate Chambers and Related Detectors, Naples, Italy (1997).

102.J. Christiansen, 32-Channel TDC with Onchip Buffering and Trigger Matching, Proceedings of Electronics for LHC experiments, London, UK (1997) 333. S. Minntoli and E. Robutti, IEEE Trans. Nucl. Sci. 47 (2000) 147.

103.P. Paolucci, The IFR Online Detector Control System, SLAC PUB-8167 (1999).

104.L. Lista, Object Oriented Reconstruction Software for the IFR Detector of BABAR Experiment, Proceedings of the Conference on Computing in High Energy Physics, Padova, Italy (2000).

105.A. Zallo et al., Nucl. Instr. Methods A456 (2000) 117.

106.A. Berenyi et al., IEEE Trans. Nucl. Sci. 46 (1999) 2006.
107.A. Berenyi et al., IEEE Trans. Nucl. Sci. 46 (1999) 348.

108.A. Berenyi et al., IEEE Trans. Nucl. Sci. 46 (1999) 928.

109.A. Berenyi et al., Nuclear Science Symposium, Conference Record 2 (1998) 988.

110.ORCA 2C series, Lucent Technologies, Berkeley Heights, NJ, USA.

111.K. Kinoshita, Nucl. Instr. Methods A276 (1989) 242.

112.Xilinx 4020E, Xilinx Inc., Mountain View, CA, USA.

113.P. D. Dauncey et al., Design and Performance of the Level 1 Calorimeter Trigger for the BABAR Detector, to be published in IEEE Trans. Nucl. Sci. (2001).

114.G.C. Fox, S. Wolfram, Nucl. Phys. B149 (1979) 413.

115.Sun Ultra 5, with single $333 \mathrm{MHz}$ UltraSPARC-IIi CPUs and 512 Mbytes of RAM, Sun Microsystems, Inc., Palo Alto, CA, USA.

116.Cisco model 6500, Cisco Systems, Inc., San Jose, CA, USA.

117.A single Sun Microsystems Ultra Enterprise 450 , with four $300 \mathrm{MHz}$ CPUs, 2 Gbytes of RAM, and 720 Gbytes of RAID-3 disk, acts as the data logging server as well as the ODF boot server. Beginning in 2001 all its responsibilities other than data logging were moved to a new Sun Ultra 220R machine with dual $450 \mathrm{MHz}$ UltraSPARC-II CPUs, 1 Gbytes of RAM, and an additional 200 Gbytes RAID array.

118.These file and database servers (presently five) are primarily additional Sun Ultra 220Rs, each with about 200 Gbytes of RAID disk. The ten application servers are a mix of Sun Ultra $60 \mathrm{~s}$, with dual $360 \mathrm{MHz}$ UltraSPARC-II CPUs and 1 Gbytes of RAM, and Ultra $5 \mathrm{~s}$; the console servers are all Ultra $5 \mathrm{~s}$.

119.R. Claus et al., Development of a Data Acquisition System for the BABAR CP Violation Experiment, Proceedings of the 11th IEEE NPSS RealTime Conference, Santa Fe, NM, USA (1999), http://strider.lansce.lanl.gov/- 
rt99/index11.html.

120.P. Grosso et al., The BABAR Fast Control System, Proceedings of the International Conference on Computing in High-Energy Physics, Chicago, IL, USA (1998).

121.J. Postel, User Datagram Protocol, RFC-0768 (1980); Internet documentation including all RFCs (Requests for Comment) are available online from the Internet Engineering Task Force, http://www.ietf .org.

122.Motorola model MVME2306 boards, each with a $300 \mathrm{MHz}$ PowerPC 604 CPU, 32 Mbytes of RAM, 5 Mbytes of non-volatile flash memory, two PCI mezzanine card slots (only one of which is currently used), a $100 \mathrm{Mbits} / \mathrm{s}$ Ethernet interface, and a Tundra Universe II VME interface, Motorola Inc., Tempe, AZ, USA.

123.G-Link, Giga-bit Transmit/Receive Chip Set HDMP-1012/HDMP-1014, Hewlett-Packard, Inc., Palo Alto, CA, USA.

124.T.J. Pavel et al., Network Performance Testing for the BABAR Event Builder, Proceedings of the CHEP Conference, Chicago, IL, USA (1998).

125.S. Deering, Host Extensions for IP Multicasting, RFC-1112 (1989).

126.G. P. Dubois-Felsmann, E. Chen, Yu. Kolomensky et al., IEEE Trans. Nucl. Sci. 47 (2000) 353.

127.S. Metzler, et al., Distributed Histogramming, Proceedings of the International Conference on Computing in High Energy Physics, Chicago, IL, USA (1998).

128.CORBA (Common Object Request Broker Architecture) Standards, Object Management Group, http://www . corba.org/.

129.ACE/TAO CORBA implementation, Distributed Object Computing Group, Washington University, St. Louis, Missouri, USA and University of California, Irvine, CA, USA, http://www.cs.wustl.edu/schmidt/TAO.html.

130.T.Johnson, Java Analysis Studio, Proceedings of the International Conference on Computing in High Energy Physics, Padova, Italy (2000).

131.High Performance Storage System (HPSS),
International Business Machines, Inc., Armonk, NY, USA.

132.The ODC IOCs are model MVME177 boards with MC68060 CPUs from Motorola Computer Group, op. cit.

133.Controller area network (CAN) for highspeed communication (1993); ISO standards: 11519 Road vehicles (Low-speed serial data communication) (1994); 11898, Road vehicles (Interchange of digital information), http://www . iso.ch/.

134.T. Meyer, R. McKay, The BABAR General Monitoring Board, BABAR Note 366 (1998).

135.B. Franek, C. Gaspar, IEEE Trans. Nucl. Sci. 45 (1998) 1946.

136.C. Gaspar, M. Donszelman, DIM - A Distibuted Information Management System for the DELPHI experiment at CERN, Proceedings of the IEEE Eighth Conference on Real-Time Computer Applications in $\mathrm{Nu}$ clear, Particle and Plasma Physics, Vancouver, Canada (1993).

137.G. Zioulas, et al., Ambient and Configuration databases for the BABAR online system, Proceedings of the IEEE Real Time Conference, Santa Fe, NM, USA (1999).

138.I. Gaponenko, et al., An Overview of the BABAR Conditions Database, Proceedings of the International Conference on Computing in High Energy Physics, Padova, Italy (2000).

139.cdev and CMLOG are software facilities produced by the Accelerator Controls group of the Thomas Jefferson National Accelerator Facility. Documentation is available from their Web site, http://www . cebaf.gov/.

140.Oracle Corporation, Redwood Shores, CA, USA.

141.The Concurrent Versions System is an opensource distributed version control system, http://www. cvshome.org/.

142.BABAR Software Release Tools, http://www.slac.stanford.edu/BFROOT/www/doc/workbook/srt1/srt1.html. 